\title{
CATEGORICAL RELATIONS BETWEEN LANGLANDS DUAL QUANTUM AFFINE ALGEBRAS: EXCEPTIONAL CASES
}

\author{
SE-JIN OH AND TRAVIS SCRIMSHAW
}

\begin{abstract}
We first compute the denominator formulas for quantum affine algebras of all exceptional types. Then we prove the isomorphisms among Grothendieck rings of categories $C_{Q}^{(t)}$ $(t=1,2,3), \mathscr{C}_{\mathscr{Q}}^{(1)}$ and $\mathscr{C}_{\mathfrak{Q}}^{(1)}$. These results give Dorey's rule for all exceptional affine types, prove the conjectures of Kashiwara-Kang-Kim and Kashiwara-Oh, and provides the partial answers of Frenkel-Hernandez on Langlands duality for finite dimensional representations of quantum affine algebras of exceptional types.
\end{abstract}

\section{Contents}

1. Introduction 2

Acknowledgements 6

2. $r$-cluster points and their folded Auslander-Reiten quivers with coordinates 6

2.1. Cartan datum and quantum groups 6

2.2. Adapted and (triply) twisted adapted points $\quad 7$

2.3. (Folded) Auslander-Reiten quivers 10

2.4. Statistics on $\widehat{\Upsilon}_{\left[\widetilde{w}_{0}\right]} \quad 13$

3. Quantum affine algebras 14

3.1. Quantum affine algebra 14

3.2. R-matrices 20

3.3. Denominator formulas and folded AR quiver 21

3.4. Dorey's rule, categorification and folded AR quiver 23

4. Denominator formulas for quantum affine algebras 25

4.1. $U_{q}^{\prime}\left(G_{2}^{(1)}\right)$ and $U_{q}^{\prime}\left(D_{4}^{(3)}\right) \quad 25$

4.2. $U_{q}^{\prime}\left(E_{6}^{(2)}\right)$ and $U_{q}^{\prime}\left(F_{4}^{(1)}\right) \quad 32$

4.3. $U_{q}^{\prime}\left(E_{6,7,8}^{(1)}\right) \quad 39$

4.4. Conclusion: Folded AR quiver and denominator formulas 43

5. Quiver Hecke algebras and generalized Schur-Weyl duality 44

5.1. Quiver Hecke algebras 44

5.2. $R$-matrices for quiver Hecke algebras $\quad 46$

5.3. Generalized Schur-Weyl duality functors 48

2010 Mathematics Subject Classification. 17B37, 17B65, 17B10, $05 \mathrm{E} 10$.

Key words and phrases. R-matrix, affine quantum group, Dorey's rule, quiver Hecke algebra.

S.-j. Oh was partially supported by the National Research Foundation of Korea(NRF) Grant funded by the Korea government(MSIP) (NRF-2016R1C1B2013135). T. Scrimshaw was partially supported by the Australian Research Council DP170102648. 
6. Application from generalized Schur-Weyl duality 50

6.1. $U_{q}^{\prime}\left(E_{6,7,8}^{(1)}\right) \quad 50$

6.2. $U_{q}^{\prime}\left(D_{4}^{(3)}\right)$ and $U_{q}^{\prime}\left(E_{6}^{(2)}\right) \quad 51$

6.3. $U_{q}^{\prime}\left(F_{4}^{(1)}\right)$ and $U_{q}^{\prime}\left(G_{2}^{(1)}\right) \quad 55$

7. Refining denominator formulas 58

$\begin{array}{ll}\text { 7.1. } U_{q}^{\prime}\left(F_{4}^{(1)}\right) & 58\end{array}$

7.2. $U_{q}^{\prime}\left(E_{6}^{(2)}\right) \quad 59$

Appendix A. Dynkin quiver $Q$ of type $E_{7}$ and its $\mathrm{AR}$ quiver $\Gamma_{Q} \quad 60$

Appendix B. Dynkin quiver $Q$ of type $E_{8}$ and its AR quiver $\Gamma_{Q} \quad 61$

Appendix C. Denominator formulas for type $E_{7}^{(1)} \quad 61$

Appendix D. Denominator formulas for type $E_{8}^{(1)} \quad 62$

Appendix E. Addendum - Removing Ambiguities 64

$\begin{array}{ll}\text { References } & 67\end{array}$

\section{INTRODUCTION}

Let $U_{q}^{\prime}(\mathfrak{g})$ be a quantum affine algebra over an algebraically closed field $\mathbf{k}$ of characteristic zero. The category $\mathcal{C}_{\mathfrak{g}}$ of finite dimensional integrable modules over $U_{q}^{\prime}(\mathfrak{g})$ has been intensively studied since it is closely related to many branches of mathematics and physics including statistical mechanics, cluster algebras, dynamical systems, etc. We refer the reader to [CP95, FR99, Her10, HL10, IKT12, Kas02, KNS11, Nak04] and references therein. The category $\mathcal{C}_{\mathfrak{g}}$ is a k-linear abelian monoidal category in the sense that it is abelian, the tensor functor $\otimes$ is k-bilinear and exact. Moreover it is rigid in the sense that each module $M$ in $\mathcal{C}_{\mathfrak{g}}$ has a left dual $M^{*}$ and a right dual ${ }^{*} M$ (see (3.3) for more details). Inside $\mathcal{C}_{\mathfrak{g}}$, there exists a rigid monoidal subcategory, denoted by $\mathcal{C}_{\mathfrak{g}}^{0}$, that contains all simple modules in $\mathcal{C}_{\mathfrak{g}}$ up to parameter shifts (see [HL10, Section 10] and [KKKO16, $\S 3.1])$.

The category $\mathcal{C}_{\mathfrak{g}}^{0}$ contains a countably infinitely set $\mathcal{P}:=\left\{V\left(\varpi_{i}\right)_{a}\right\}_{\left(i \in I, a \in \mathbb{Z}\left[q_{s}^{ \pm 1}\right]\right)}$ consisting of simple $U_{q}^{\prime}(\mathfrak{g})$-modules called the fundamental modules with spectral parameters $a$, whose tensor monomials in a certain order form a basis of the Grothendieck ring $\left[\mathcal{C}_{\mathfrak{g}}^{0}\right]$ of $\mathcal{C}_{\mathfrak{g}}^{0}$ ([AK97, Cha02, Kas02, VV02]). Thus one can understand the set $\mathcal{P}$ as PBW-type modules of $\mathcal{C}_{\mathfrak{g}}^{0}$.

For modules $M, N \in \mathcal{C}_{\mathfrak{g}}$, there exists a non-zero $U_{q}^{\prime}(\mathfrak{g})$-homomorphism

$$
\mathbf{r}_{M, N}: M \otimes N \rightarrow N \otimes M
$$

that satisfies the Yang-Baxter equation. When $M$ and $N$ are good modules in the sense of [Kas02], there exists a polynomial called the denominator $d_{M, N}(z) \in \mathbf{k}[z]$. The roots of $d_{M, N}(z)$ determine whether $\mathbf{r}_{M_{a}, N_{b}}$ is an isomorphism or not. Indeed, the subcategory $\mathcal{C}_{\mathfrak{g}}^{0}$ and the order in the previous paragraph are determined by the denominator formulas $d_{i, j}(z):=d_{V\left(\varpi_{i}\right), V\left(\varpi_{j}\right)}(z)$ between the fundamental modules $V\left(\varpi_{i}\right)$ and $V\left(\varpi_{j}\right)$. Roughly speaking, one can say that the representation theory for $\mathcal{C}_{\mathfrak{g}}$ is controlled by the denominator formulas $d_{i, j}(z)$.

For $\mathfrak{g}^{(1)}$ of type $A_{n}^{(1)}, D_{n}^{(1)}$ or $E_{6,7,8}^{(1)}$ in [HL15] and $\mathfrak{g}^{(2)}$ of type $A_{n}^{(2)}, D_{n}^{(2)}$ or $E_{6}^{(2)}$ in [KKKO16, Oh19], a tensor subcategory $\mathcal{C}_{Q}^{(t)}$ of $\mathcal{C}_{\mathfrak{g}}^{0}$, where $t=1,2$ and $\mathfrak{g}=\mathfrak{g}^{(t)}$, that contains finite number 
of fundamental modules $\mathcal{P}_{Q}^{(t)}:=\left\{V_{Q}^{(t)}(\beta):=V\left(\varpi_{i_{\beta}}\right)_{a_{\beta}} \mid \beta \in \Phi^{+}\right\} \subset \mathcal{P}$ whose tensor monomials in the induced order (in short, PBW-monomials) form a basis of $\left[\mathcal{C}_{Q}^{(1)}\right]$ and whose repeated duals of $V_{Q}^{(t)}(\beta)$ 's cover $\mathcal{P}$ :

$$
\mathcal{P} \ni M \Longleftrightarrow \text { there exists } \beta \in \Phi^{+} \text {and } k \in \mathbb{Z} \text { such that } M=\left(V_{Q}^{(t)}(\beta)\right)^{k *} .
$$

Here $Q$ denotes a Dynkin quiver of the corresponding finite type Dynkin diagram and $\Phi^{+}$is the set of positive roots of the same finite type. Also the pair $\left(i_{\beta}, a_{\beta}\right) \in I \times \mathbb{Z}\left[q_{s}^{ \pm 1}\right]$ for each $V_{Q}^{(t)}(\beta)$ is determined by the coordinate system of the Auslander-Reiten (AR) quiver $\Gamma_{Q}$ of the path algebra $\mathbb{C} Q$. Furthermore, it was shown in [HL15, KKKO16] that $\mathcal{C}_{Q}^{(t)}$ categorifies the negative half $U_{q}^{-}(\mathrm{g})$ of the finite quantum group $U_{q}(\mathrm{~g}) \subset U_{q}^{\prime}(\mathfrak{g})$ and PBW-monomials in $\mathcal{P}_{Q}^{(t)}$ arranged by $d_{i, j}(z)$ categorify the PBW-basis of $U_{q}^{-}(\mathrm{g})$ arising from $Q$. However, for $t=2$, the result depends on Dorey's rule, which is only conjectured for type $E_{6}^{(2)}$.

Let us briefly recall a combinatorial property of $\Gamma_{Q}$ (see [B9́9]). For a finite Weyl group $W$ of the same type of $Q$, let $w_{0}$ be the longest element and $\widetilde{w}_{0}$ be a reduced expression of $w_{0}$. Then $\Gamma_{Q}$ is the Hasse diagram of the convex partial order $\prec_{Q}$ on $\Phi^{+}$. In particular, each $\prec_{Q}$ coincides with the convex partial order $\prec_{[Q]}$ induced from the commutation class $[Q]$ consisting of all reduced expressions adapted to $Q$ and has its unique Coxeter element. Also all commutation classes adapted to some $Q$ can be grouped into one $r$-cluster point.

In [Oh16, Oh17], the first named author observed that if $V_{Q}^{(t)}(\alpha) \otimes V_{Q}^{(t)}(\beta)$ is reducible, then $(\alpha, \beta)$ are comparable with respect to $\prec_{Q}$ when $\mathfrak{g}$ is of type $A_{n}^{(t)}$ or $D_{n}^{(t)}(t=1,2)$. The observation means that the order for PBW-monomials in $\mathcal{P}_{Q}^{(t)}(t=1,2)$ determined by $d_{i, j}(z)$ is equivalent to $\prec_{Q}$. Furthermore, the following are proved:

(1) The conditions for

$$
\operatorname{Hom}_{U_{q}^{\prime}(\mathfrak{g})}\left(V\left(\varpi_{i}\right)_{a} \otimes V\left(\varpi_{j}\right)_{b}, V\left(\varpi_{k}\right)_{c}\right) \neq 0
$$

can be interpreted as the coordinates in some $\Gamma_{Q}$ when $\mathfrak{g}$ is of type $A_{n}^{(t)}$ or $D_{n}^{(t)}$ and are referred to as Dorey's rule for quantum affine algebras.

(2) By defining new statistics on $\Gamma_{Q}$, the denominator formulas for $A_{n}^{(t)}$ or $D_{n}^{(t)}$ can be read from any $\Gamma_{Q}$.

Here let us review the history of Dorey's rule briefly. In [Dor91], Dorey described relations between three-point couplings in the simply-laced affine Toda field theories (ATFTs) and Lie theories. More precisely, simply-laced ATFTs on untwisted affine Lie algebras are related to the same type of Lie algebras and simply-laced ATFTs on twisted affine Lie algebras are related to non-simply laced Lie algebras obtained by corresponding Dynkin diagram automorphisms. Generally, in ATFTs, quantum affine algebras appear as quantum symmetry groups and the fundamental representations of quantum affine algebras correspond to the quantum particles [BL91]. Interestingly, Dorey's rule was interpreted by Chari and Pressley [CP96] in the language of representation theory of classical quantum affine algebras by using Coxeter elements for $U_{q}^{\prime}\left(A_{n}^{(1)}\right)$ and $U_{q}^{\prime}\left(D_{n}^{(1)}\right)$ and twisted Coxeter elements for $U_{q}^{\prime}\left(B_{n}^{(1)}\right)$ and $U_{q}^{\prime}\left(C_{n}^{(1)}\right)$. Analogous results for $U_{q}^{\prime}\left(E_{6,7,8}^{(1)}\right)$ and classical twisted affine types were shown in $\left[\mathrm{FH}^{+} 15\right.$, Section 7.3], [Oh19] and [KKKO16] respectively (see also [YZ11]). 
Motivated by the ideas of Chari and Pressley on Dorey's rule for quantum affine algebras of type $B_{n}^{(1)}$ (resp. $C_{n}^{(1)}$ ) using twisted Coxeter elements of type $A_{2 n-1}$ (resp. $D_{n+1}$ ), the first named author and Suh introduced the folded AR quiver $\widehat{\Upsilon}_{\mathscr{Q}}$ in [OS15, OS16a, OS16b], a combinatorial object that can be understood as a twisted analogue of $\Gamma_{Q}$. Here [[Q2]] denotes the $r$-cluster point of a simply-laced finite type $X$ with positive roots $\Phi^{+}$, which corresponds to any twisted Coxeter element. In particular, the folded AR quiver has the following properties:

(1) $\widehat{\Upsilon}_{\mathscr{Q}}$ is the Hasse diagram of $\prec_{[\mathscr{Q}]}$.

(2) We can define some certain subcategory $\mathscr{C}_{\mathscr{Q}}^{(1)}$ of $\mathcal{C}_{\mathfrak{g}}$ for $\mathfrak{g}=B_{n}^{(1)}, C_{n}^{(1)}, F_{4}^{(1)}$ and $G_{2}^{(1)}$ determined by the coordinate system of $\widehat{\Upsilon}_{\mathscr{Q}}$ (see also the category $\mathscr{C}^{-}$in [HL16]).

(3) The modules $\mathscr{P}_{\mathscr{Q}}:=\left\{V_{\mathscr{Q}}(\beta) \in \mathscr{C}_{\mathscr{Q}}^{(1)} \mid \beta \in \Phi^{+}\right\}$satisfies the property stated in (1.1).

(4) When $\mathfrak{g}=B_{n}^{(1)}$ or $C_{n}^{(1)}$, the order for PBW-monomials in $\mathscr{P}_{\mathscr{Q}}$ arranged by $d_{i, j}(z)$ is equivalent to $\prec_{[Q 2]}$.

(5) For $\mathfrak{g}=B_{n}^{(1)}$ or $C_{n}^{(1)}$, the Dorey's rule can be interpreted as [Q]-minimal pair $(\alpha, \beta)$ of $\alpha+\beta \in \Phi^{+}$for some [Q2], and the denominator formulas can be read from any $\widehat{\Upsilon}_{\mathscr{Q}}$.

However, due to the lack of Dorey's rule and denominator formulas for all exceptional affine types, there were not exceptional affine types analogues of many of the aforementioned results.

The quiver Hecke algebras $R^{\mathrm{g}}$, independently introduced by Khovanov-Lauda [KL09, KL11] and Rouquier [Rou08], categorify the negative part $U_{q}^{-}(\mathrm{g})$ of quantum groups $U_{q}(\mathrm{~g})$ for all symmetrizable Kac-Moody algebras g. The categorification for (dual) PBW-bases and global bases of the integral form $U_{\mathbb{A}}^{-}(\mathrm{g})$ of $U_{q}^{-}(\mathrm{g})$ were very quickly developed since the introduction of quiver Hecke algebras. Among them, [BKM14, Kat14, KR11, McN15] give the categorification theory for (dual) PBWbases of $U_{\mathbb{A}}^{-}(\mathrm{g})$ associated to finite simple Lie algebra $\mathrm{g}$ by using convex orders $\prec_{\left[\widetilde{w}_{0}\right]}$ on the set of positive roots $\Phi^{+}$for any commutation class $\left[\widetilde{w}_{0}\right]$. As we reviewed, $U_{q}^{-}(\mathrm{g})$ for finite type $A_{n}, D_{n}$ or $E_{6,7,8}$ is also categorified by $\mathcal{C}_{Q}^{(1)}$. Thus it was natural to ask the concrete relationship between the categories $\operatorname{Rep}\left(R^{\mathrm{g}}\right)$ and $C_{Q}^{(1)}$ (see $\left.[\mathrm{HL} 15, \S 1.6]\right)$.

When $\mathfrak{g}$ is of affine type $A_{n}^{(1)}$ and $D_{n}^{(1)}$, the affirmative answer was given by Kang, Kashiwara and Kim in [KKK15, KKK18]: For a quantum affine algebra $U_{q}^{\prime}(\mathfrak{g})$, we can construct a generalized Schur-Weyl duality functor

$$
\mathcal{F}: \operatorname{Rep}\left(R^{J}\right) \rightarrow \mathcal{C}_{\mathfrak{g}}
$$

by observing denominator formulas $d_{V, W}(z)$ between good modules $V, W \in \mathcal{C}_{\mathfrak{g}}$. Here $R^{J}$ is the quiver Hecke algebra determined by only the choice of good modules in $\mathcal{C}_{\mathfrak{g}}$ and denominator formulas. For $\mathfrak{g}$ of affine type $A_{n}^{(1)}$ and $D_{n}^{(1)}$, by taking $\left\{V_{Q}^{(1)}\left(\alpha_{i}\right)\right\}_{i \in I}$ as the chosen good modules, they proved that the quiver Hecke algebra $R^{J}$ is indeed $R^{\mathrm{g}}$ by using the denominator formulas $d_{i, j}(z)$ for $U_{q}^{\prime}(\mathfrak{g})$. Moreover, by adopting the framework of [HL15], it was shown that the induced generalized SchurWeyl duality functor

$$
\mathcal{F}_{Q}^{(1)}: \operatorname{Rep}\left(R^{\mathrm{g}}\right) \rightarrow \mathcal{C}_{Q}^{(1)}
$$

sends simple modules to simple modules bijectively. Thus the PBW-type modules associated to $[Q]$ in both categories can be identified via $\mathcal{F}_{Q}^{(1)}$. These results were extended to the cases $\mathcal{C}_{Q}^{(2)}$ for $\mathfrak{g}=A_{n}^{(2)}$ and $D_{n}^{(2)}$ in [KKKO16]. In these cases, however, to show the bijection, the Dorey's rule for $U_{q}^{\prime}(\mathfrak{g})$, developed in [KKKO16], was crucially used instead of [HL15]. 
Since there are $\mathscr{C}_{\mathscr{Q}}, \widehat{\Upsilon}_{\mathscr{Q}}$, Dorey's rule and denominator formulas for $\mathfrak{g}=B_{n}^{(1)}$ and $C_{n}^{(1)}$, the above results were extended to the $U_{q}^{\prime}(\mathfrak{g})$ cases [KO18]. Here, [Q2] denotes a commutation class of type $A_{2 n-1}$ or $D_{n+1}$, respectively, arising from a twisted Coxeter element associated to the corresponding Dynkin diagram folding. More precisely, there exists an exact generalized Schur-Weyl duality functor

$$
\mathscr{F}_{\mathscr{Q}}: \operatorname{Rep}\left(R^{\mathrm{g}}\right) \rightarrow \mathscr{C}_{\mathscr{Q}}^{(1)}, \quad(\mathrm{g}, \mathfrak{g})=\left\{\begin{array}{l}
\left(A_{2 n-1}, B_{n}^{(1)}\right), \\
\left(D_{n+1}, C_{n}^{(1)}\right),
\end{array}\right.
$$

which sends simple modules to simple modules bijectively, and give a categorification theorem for the PBW-bases of $U_{q}^{-}(\mathrm{g})$ associated to $[\mathscr{Q}]$ via the PBW-monomials in $\mathscr{P}_{\mathscr{Q}}$.

Hence, there are subcategories $\mathscr{C}_{Q}^{(2)}$ of $U_{q}^{\prime}\left(\mathfrak{g}^{(2)}\right)$-modules $\left(\mathfrak{g}^{(2)}=A_{2 n-1}^{(2)}, D_{n+1}^{(2)}\right)$ and $\mathscr{C}_{\mathscr{Q}}$ of $U_{q}^{\prime}\left({ }^{L} \mathfrak{g}^{(2)}\right)$ modules $\left({ }^{L} \mathfrak{g}^{(2)}=B_{n}^{(1)}, C_{n}^{(1)}\right)$ whose Grothendieck groups are isomorphic to each other. Such similarities between twisted quantum affine algebra and its untwisted Langlands dual were observed by many mathematician and are known to be related to the geometric Langlands correspondence (see [FH11a, FH11b, FR98]).

In this paper, we extend these results to all exceptional affine algebras $U_{q}^{\prime}(\mathfrak{g})$. As previously mentioned, the main difficulties we had to overcame were lack of denominator formulas and Dorey's rule for exceptional affine cases. In particular, the denominator formulas for exceptional types are quite difficult to study due to their large dimensions and multiplicities in their classical decompositions. In order to compute the denominator formulas, we required an explicit basis for $V\left(\varpi_{i}\right)$ with the $U_{q}^{\prime}(\mathfrak{g})$-action on that basis. To the best knowledge of the authors, this was not known. We began by constructing an explicit $U_{q}^{\prime}(\mathfrak{g})$-module structure for fundamental modules associated to minuscule and adjoint representations by using the corresponding crystal graphs. Using this, we can compute the denominator formulas between these representations and the induced Dorey's type homomorphisms. This allows us to compute all denominator formulas $d_{i, j}(z)$ by applying the framework of $[\mathrm{Oh} 15]$ and statistics on $\Gamma_{Q}$ and $\widehat{\Upsilon}_{\mathscr{Q}}$.

From the denominator formulas $d_{i, j}(z)$, we can construct exact functors

$$
\mathcal{F}_{Q}^{(t)}: \operatorname{Rep}\left(R^{\mathrm{g}}\right) \rightarrow \mathcal{C}_{Q}^{(t)}(t=1,2,3), \quad \mathscr{F}_{\mathscr{Q}}: \operatorname{Rep}\left(R^{\mathrm{g}}\right) \rightarrow \mathscr{C}_{\mathscr{Q}}^{(1)} .
$$

This gives an answer for [KKK15, Conjecture 4.3.2]. This conjecture for $E_{6,7,8}^{(1)}$ is also proved by Fujita [Fuj18], announced after completing this paper, by generalizing Ginzburg-ReshetikhinVasserot's geometric realization of the quantum affine Schur-Weyl duality [GRV94] to generalized Schur-Weyl duality for $A_{n}^{(1)}, D_{n}^{(1)}$ and $E_{6,7,8}^{(1)}$, which also implies simpleness of some roots of the denominator formulas in that cases.

By utilizing both categories with the exact functors, we can obtain the complete Dorey's rules for all exceptional affine cases. In particular, we use the rigidity of $\mathcal{C}_{\mathfrak{g}}$ and the exact sequences for ([Q2]-minimal) pairs $(\alpha, \beta)$ of $\alpha+\beta \in \Phi^{+}$in $\operatorname{Rep}\left(R^{\mathrm{g}}\right)$. With the results on denominator formulas and Dorey's type homomorphisms at hand, we prove that

(1) $\mathcal{C}_{Q}^{(t)}$ and $\mathscr{C}_{\mathscr{Q}}^{(1)}$ give categorifications of $U_{q}^{-}(\mathrm{g})$,

(2) $\mathcal{F}_{Q}^{(t)}$ and $\mathscr{F}_{\mathscr{Q}}$ sends simple modules to simple modules bijectively, and

(3) the PBW-monomials in $\mathcal{P}_{Q}^{(t)}$ and $\mathscr{P}_{\mathscr{Q}}$ give categorifications of the PBW bases of $U_{q}^{-}(\mathrm{g})$ associated to $[Q]$ and $[\mathscr{Q}]$ for all exceptional cases.

These results for $E_{6}^{(t)}(t=1,2)$ and $F_{4}^{(1)}$ were conjectured in [KO18, Appendix]. 
Now we have the following diagram which are related to our works on exceptional cases:
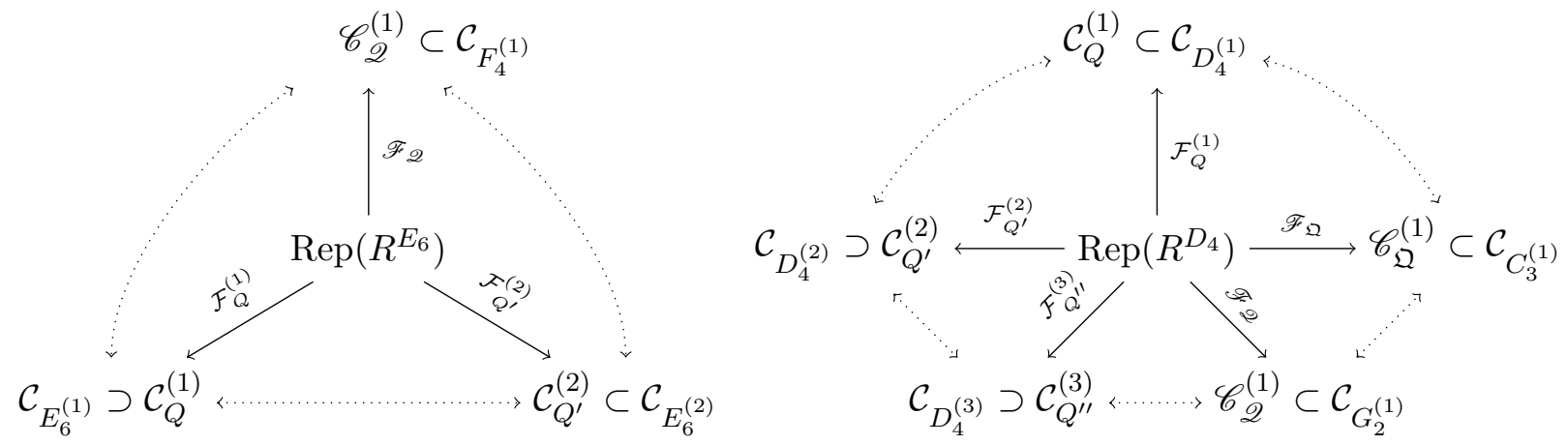

These diagrams are closely related to the conjectures of Frenkel-Hernandez (see [FH11b, Conj. 2.2, Conj. 2.4, Conj. 3.10]) for exceptional affine types similar to classical affine types as shown in [KO18]: For a representation $V$ in $\mathcal{C}_{Q}^{(2)}$, one can corresponds to a representation $\widehat{V}$ in $\mathscr{C}_{\mathscr{Q}}^{(1)}$ in a canonical way via induced functor $\mathcal{F}_{\mathscr{Q}} \circ\left(F_{Q}^{(2)}\right)^{-1}$ for any $[Q]$ and [QQ $]$. Furthermore, if $V$ is simple, so is $\widehat{V}$.

Therefore, our results finish this program on categorical similarities between monoidal subcategories of (un)twisted quantum affine algebras and their Langlands duals for the exceptional affine types, where the nonexceptional affine types were done in [KKK15, KKKO16, KO18] (see also $[\mathrm{KKO} 17])$.

This paper is organized as follows. In Section 2, we briefly review the previous results on the commutations classes and (folded) AR quivers we require. In Section 3, we review the necessary theory on quantum affine algebras, Dorey's rule, denominator formulas and (folded) AR quivers. We also give our explicit $U_{q}^{\prime}(\mathfrak{g})$-module structure for affine minuscule and adjoint representations. In Section 4, we compute the denominator formulas for all exceptional types with some ambiguity for roots of higher orders and parts of Dorey's type homomorphisms. In Section 5, we review the basic materials on quiver Hecke algebras, the generalized Schur-Weyl duality functors and known results on the functors. In Section 6, we apply the denominator formulas obtained in Section 4 to the context of the generalized Schur-Weyl duality, which yields our main results for categories and Dorey's rule. In Section 7 and Appendix E, we remove all of ambiguities about orders of roots in cases of $U_{q}^{\prime}\left(F_{4}^{(1)}\right), U_{q}^{\prime}\left(E_{6}^{(2)}\right)$ and $E_{6,7,8}^{(1)}$ by using the exact functor what have constructed in Section 6 and the homomorphisms investigated in [Oh19].

Note that Appendix E has been included as an addendum to the published version [OS19], which removes all of the ambiguities of orders of roots.

Acknowledgements. The authors would like to thank Masaki Kashiwara for useful discussions. The authors would like to thank the anonymous referee for useful comments. T.S. would like to thank Ewha Womans University for its hospitality during his stay in June, 2017, where this work began. S.-j.O. would like to thank The University of Queensland for its hospitality during his stay in February, 2018. This work benefited from computations using SageMath [Dev17, SCc08].

\section{2. $r$-CLUSTER POINTS AND THEIR FOLDED AUSLANDER-REITEN QUIVERS WITH COORDINATES}

2.1. Cartan datum and quantum groups. A Cartan datum is a sextuple $\left(\mathrm{A}, P, \Pi, P^{\vee}, \Pi^{\vee},(\cdot, \cdot)\right)$ with index set $I$ consisting of (a) an integer-valued matrix $\mathrm{A}=\left(a_{i j}\right)_{i, j \in I}$, called the symmetrizable generalized Cartan matrix, (b) a free abelian group $P$ called the weight lattice, (c) $\Pi=\left\{\alpha_{i} \in P \mid\right.$ 
$i \in I\}$ called the simple roots, (d) $P^{\vee}:=\operatorname{Hom}(P, \mathbb{Z})$ called the co-weight lattice with the canonical pairing $\langle\cdot, \cdot\rangle$, (e) $\Pi^{\vee}=\left\{h_{i} \mid i \in I\right\} \subset P^{\vee}$ called the simple coroots, (f) a symmetric bilinear form $(\cdot, \cdot): P \times P \rightarrow \mathbb{Q}$, satisfying (i) $\left\langle h_{i}, \alpha_{j}\right\rangle=a_{i j}$ for all $i, j \in I$, (ii) $\Pi$ is linearly independent, (iii) for each $i \in I$, there exists $\Lambda_{i} \in P$ such that $\left\langle h_{j}, \Lambda_{i}\right\rangle=\delta_{i j}$ for all $j \in I$, (iv) $\left(\alpha_{i}, \alpha_{i}\right) \in \mathbb{Q}_{>0}$ for any $i \in I,(\mathrm{v})$ for any $\lambda \in P$ and $i \in I,\left\langle h_{i}, \lambda\right\rangle=\frac{2\left(\alpha_{i}, \lambda\right)}{\left(\alpha_{i}, \alpha_{i}\right)}$.

We call $\Lambda_{i}$ the fundamental weights. The free abelian group $Q:=\bigoplus_{i \in I} \mathbb{Z} \alpha_{i}$ is called the root lattice. Set $\mathrm{Q}^{+}=\sum_{i \in I} \mathbb{Z}_{\geq 0} \alpha_{i} \subset \mathrm{Q}$ and $\mathrm{Q}^{-}=\sum_{i \in I} \mathbb{Z}_{\leq 0} \alpha_{i} \subset \mathrm{Q}$. For $\beta=\sum_{i \in I} n_{i} \alpha_{i} \in \mathrm{Q}^{+}$, we set $|\beta|=\sum_{i \in I} n_{i} \in \mathbb{Z}_{\geq 0}$

Let $\gamma$ be the smallest positive integer such that $\gamma \frac{\left(\alpha_{i}, \alpha_{i}\right)}{2} \in \mathbb{Z}$ for all $i \in I$. For each $i \in I$, set $q_{i}=q^{\frac{\left(\alpha_{i}, \alpha_{i}\right)}{2}} \in \mathbb{Q}\left(q^{1 / \gamma}\right)$. For $m, n \in \mathbb{Z}_{\geq 0}$ and $i \in I$, we define

$$
[n]_{i}=\frac{q_{i}^{n}-q_{i}^{-n}}{q_{i}-q_{i}^{-1}}, \quad[n]_{i} !=\prod_{k=1}^{n}[k]_{i}, \quad\left[\begin{array}{c}
m \\
n
\end{array}\right]_{i}=\frac{[m]_{i} !}{[m-n]_{i} ![n]_{i} !} .
$$

Remark 2.1. The imaginary number $i$ will always be denoted by $\sqrt{-1}$ so that there is no danger of confusion with an indexing variable $i$.

Definition 2.2. For Cartan datum $\left(\mathrm{A}, P, \Pi, P^{\vee}, \Pi^{\vee},(\cdot, \cdot)\right)$, the associated (Drinfel'd-Jimbo) quantum group $U_{q}(\mathrm{~g})$ is the $\mathbb{Q}\left(q^{1 / \gamma}\right)$-algebra generated by $e_{i}, f_{i}(i \in I)$ and $q^{h}\left(h \in \gamma^{-1} P\right)$ subject to the following relations:

(i) $q^{0}=1, q^{h} q^{h^{\prime}}=q^{h+h^{\prime}}$ for $h, h^{\prime} \in \gamma^{-1} P^{\vee}$,

(ii) $q^{h} e_{i} q^{-h}=q^{\left\langle h, \alpha_{i}\right\rangle} e_{i}, q^{h} f_{i} q^{-h}=q^{-\left\langle h, \alpha_{i}\right\rangle} f_{i}$ for $h \in \gamma^{-1} P^{\vee}, i \in I$,

(iii) $e_{i} f_{j}-f_{j} e_{i}=\delta_{i j} \frac{K_{i}-K_{i}^{-1}}{q_{i}-q_{i}^{-1}}, \quad$ where $K_{i}=q_{i}^{h_{i}}$,

(iv) $\sum_{k=0}^{1-a_{i j}}(-1)^{k} e_{i}^{\left(1-a_{i j}-k\right)} e_{j} e_{i}^{(k)}=\sum_{k=0}^{1-a_{i j}}(-1)^{k} f_{i}^{\left(1-a_{i j}-k\right)} f_{j} f_{i}^{(k)}=0 \quad$ for $i \neq j$,

where $e_{i}^{(k)}=e_{i}^{k} /[k]_{i}$ ! and $f_{i}^{(k)}=f_{i}^{k} /[k]_{i}$ !. Recall that $\delta_{i j}$ is the Kronecker delta. We set $q_{s}=q^{1 / \gamma}$.

We denote by $U_{q}^{+}(\mathrm{g})$ (resp. $\left.U_{q}^{-}(\mathrm{g})\right)$ by the subalgebra of $U_{q}(\mathrm{~g})$ generated by $e_{i}$ (resp. $\left.f_{i}\right)(i \in I)$. We also denote by $U_{\mathbb{A}}^{+}(\mathrm{g})\left(\right.$ resp. $\left.U_{\mathbb{A}}^{-}(\mathrm{g})\right)$ by the $\mathbb{A}:=\mathbb{Z}\left[q, q^{-1}\right]$-subalgebra of $U_{q}(\mathrm{~g})$ generated by $e_{i}^{(n)}$ (resp. $f_{i}^{(n)}$ ) for all $i \in I$ and $n \in \mathbb{Z}$.

2.2. Adapted and (triply) twisted adapted points. Let us denote by $\Delta$ the Dynkin diagrams of finite type, labeled by an index set $I$, and their automorphisms $\sigma$. By $\sigma$, we can obtain the Dynkin diagrams $\widehat{\Delta}$ of finite type $B C F G$ as orbits of $\sigma$ :

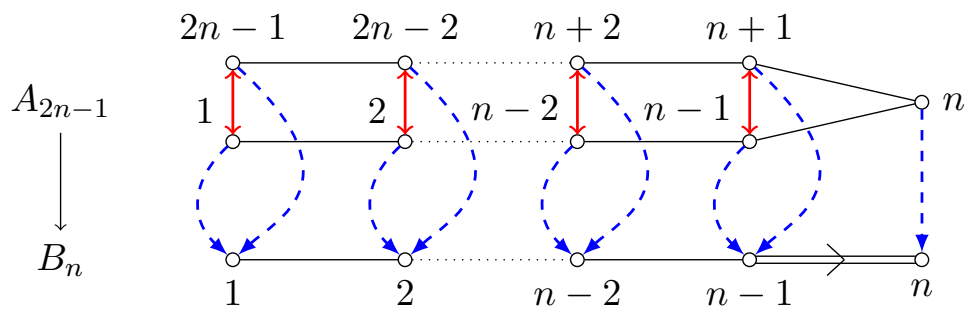



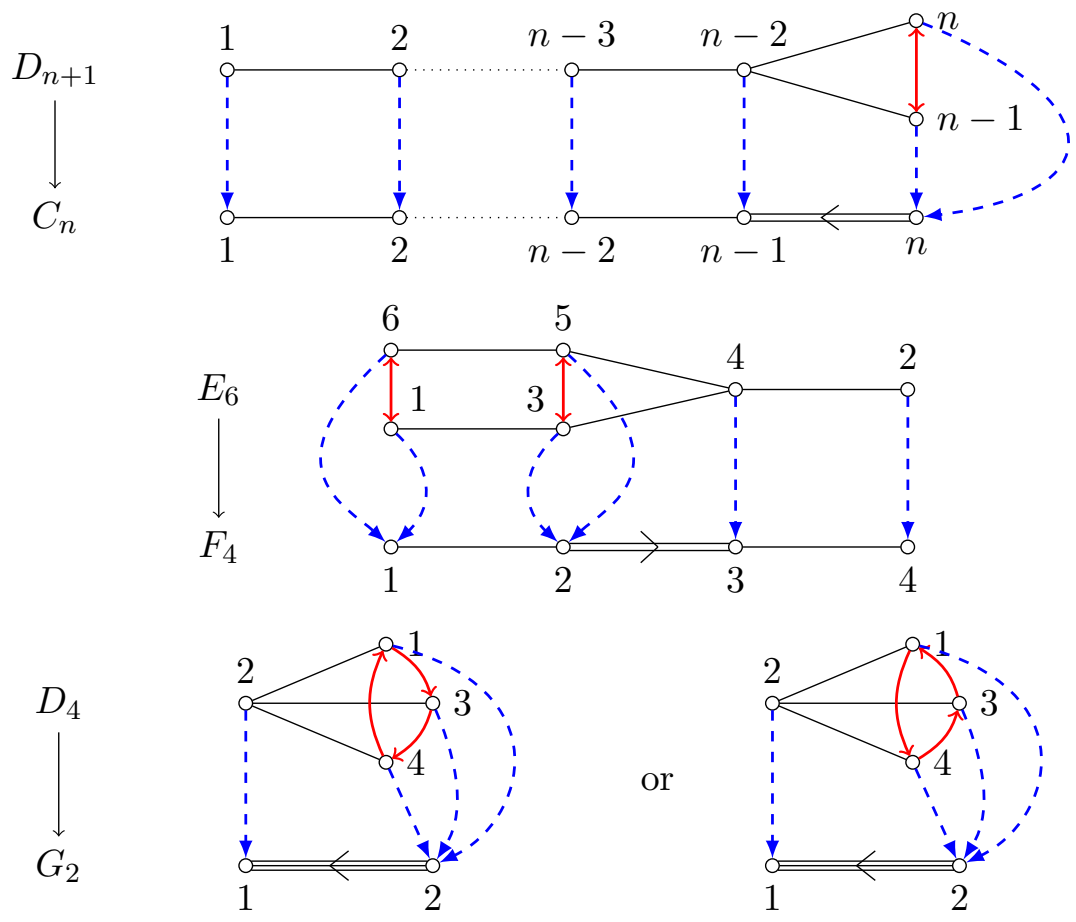

Definition 2.3. We denote by $\bar{\sigma}$ the map from vertices of $\Delta$ to the one of $\widehat{\Delta}$ induced by $\sigma$.

For example, $\bar{\sigma}(4)=3$ when $\Delta$ is of type $E_{6}$.

Let $\mathrm{W}$ be the Weyl group, generated by simple reflections $\left(s_{i} \mid i \in I\right)$ corresponding to $\Delta$, and $w_{0}$ the longest element of $\mathrm{W}$. We denote by $*$ the involution on $I$ defined by

$$
w_{0}\left(\alpha_{i}\right)=-\alpha_{i^{*}} .
$$

We also denote by $\Phi$ the set of all roots and by $\Phi^{+}$the set of all positive roots. Let $N=\left|\Phi^{+}\right|$.

Remark 2.4. In this paper, we fix the length $\left|\alpha_{i}\right|$ of the longest root as 1. Furthermore, $q_{s}=q_{i}$, where $\alpha_{i}$ is a shortest root.

We say that two reduced expressions $\widetilde{w}=s_{i_{1}} s_{i_{2}} \cdots s_{i_{\ell}}$ and $\widetilde{w}^{\prime}=s_{j_{1}} s_{j_{2}} \cdots s_{j_{\ell}}$ of $w \in W$ are commutation equivalent, denoted by $\widetilde{w} \sim \widetilde{w}^{\prime}$, if $s_{j_{1}} s_{j_{2}} \cdots s_{j_{\ell}}$ is obtained from $s_{i_{1}} s_{i_{2}} \cdots s_{i_{\ell}}$ by applying the commutation relations $s_{k} s_{l}=s_{l} s_{k}$. We denote by $[\widetilde{w}]$ the commutation equivalence class of $\widetilde{w}$.

The following proposition is well-known:

Proposition 2.5. For $\widetilde{w}_{0}=s_{i_{1}} s_{i_{2}} \cdots s_{i_{\mathrm{N}-1}} s_{i_{\mathrm{N}}}, \widetilde{w}_{0}^{\prime}=s_{i_{\mathrm{N}}^{*} s_{i_{1}}} s_{i_{2}} \cdots s_{i_{\mathrm{N}-1}}$ is a reduced expression of $w_{0}$ and $\left[\widetilde{w}_{0}^{\prime}\right] \neq\left[\widetilde{w}_{0}\right]$. Similarly, $\widetilde{w}_{0}^{\prime \prime}=s_{i_{2}} \cdots s_{i_{\mathrm{N}-1}} s_{i_{\mathrm{N}}} s_{i_{1}^{*}}$ is a reduced expression of $w_{0}$ and $\left[\widetilde{w}_{0}^{\prime \prime}\right] \neq\left[\widetilde{w}_{0}\right]$.

The action of the reflection functor $r_{i}$ on $\left[\widetilde{w}_{0}\right]$ is defined by

$$
r_{i}\left[\widetilde{w}_{0}\right]= \begin{cases}{\left[\left(s_{i^{*}}, s_{i_{1}} \cdots, s_{i_{\mathrm{N}-1}}\right)\right]} & \text { if there is } \widetilde{w}_{0}^{\prime}=\left(s_{i_{1}}, \cdots, s_{i_{\mathrm{N}-1}}, s_{i}\right) \in\left[\widetilde{w}_{0}\right], \\ {\left[\widetilde{w}_{0}\right]} & \text { otherwise. }\end{cases}
$$

Definition 2.6 ([OS15]). Let $\left[\widetilde{w}_{0}\right]$ and $\left[\widetilde{w}_{0}^{\prime}\right]$ be two commutation classes. We say $\left[\widetilde{w}_{0}\right]$ and $\left[\widetilde{w}_{0}^{\prime}\right]$ are reflection equivalent and write $\left[\widetilde{w}_{0}\right] \stackrel{r}{\sim}\left[\widetilde{w}_{0}^{\prime}\right]$ if $\left[\widetilde{w}_{0}^{\prime}\right]$ can be obtained from $\left[\widetilde{w}_{0}\right]$ by a sequence of 
reflection maps. The equivalence class $\llbracket \widetilde{w}_{0} \rrbracket:=\left\{\left[\widetilde{w}_{0}\right] \mid\left[\widetilde{w}_{0}\right] \stackrel{r}{\sim}\left[\widetilde{w}_{0}^{\prime}\right]\right\}$ with respect to the reflection equivalence relation is called an $r$-cluster point.

Let $\sigma \in G L(\mathbb{C} \Phi)$ be a linear transformation of finite order which preserves $\Pi$. Hence $\sigma$ preserves $\Phi$ itself and normalizes $\mathrm{W}$ and so $\mathrm{W}$ acts by conjugation on the coset $W \sigma$. Note that $\sigma$ agrees with the Dynkin diagram automorphism given by (2.1).

Let $\left\{\Pi_{i_{1}}, \ldots, \Pi_{i_{k}}\right\}$ be the all orbits of $\Pi$ in $\Phi$ with respect to $\sigma$. For each $r \in\{1, \cdots, k\}$, choose $\alpha_{i_{r}} \in \Pi_{i_{r}}$ arbitrarily, and let $s_{i_{r}} \in W$ denote the corresponding reflection. Let $w$ be the product of $s_{i_{1}}, \ldots, s_{i_{k}}$ in any order. The element $w \sigma \in W \sigma$ of $w \in W$ thus obtained is called a $\sigma$-Coxeter element.

- If $\sigma=\mathrm{id}$, then a id-Coxeter element is simply called a Coxeter element.

- If $\sigma$ is an involution, then a $\sigma$-Coxeter element is also called a twisted Coxeter element.

- If $\sigma$ is of order 3, then a $\sigma$-Coxeter element is also called a triply twisted Coxeter element.

\section{Example 2.7.}

(1) For $\sigma$ in (2.1c), $s_{1} s_{3} s_{4} s_{2} \sigma$ is a twisted Coxeter element of finite type $E_{6}$.

(2) For $\sigma$ in (2.1d), $s_{2} s_{1} \sigma$ is a triply twisted Coxeter element of finite type $D_{4}$.

For a reduced expression $s_{j_{1}} \cdots s_{j_{\ell}}$, we define

$$
\left(s_{j_{1}} \cdots s_{j_{\ell}}\right)^{\sigma}:=s_{j_{1}^{\sigma}} \cdots s_{j_{\ell}^{\sigma}} \text { and }\left(s_{j_{1}} \cdots s_{j_{s}}\right)^{k \sigma}:=\left(\cdots \left((s_{j_{1}} \cdots s_{j_{s}} \underbrace{)^{\sigma}\right)^{\sigma} \cdots\right)^{\sigma}}_{k \text {-times }} .\right.\right.
$$

Proposition 2.8 ([OS16a, OS16b]). For each (triply) twisted Coxeter element $s_{i_{1}} \cdots s_{i_{\ell}} \sigma$,

$$
\widetilde{w}_{0}^{\sigma}:=\prod_{i=0}^{\left(\left|\Phi^{+}\right| / \ell\right)-1}\left(s_{i_{1}} \cdots s_{i_{\ell}}\right)^{k \sigma} \text { is a reduced expression of } w_{0} .
$$

\section{Definition 2.9.}

(1) For $\sigma$ in $(2.1 \mathrm{a}),(2.1 \mathrm{~b}),(2.1 \mathrm{c}), \llbracket \mathscr{Q} \rrbracket:=\llbracket \widetilde{w}_{0}^{\sigma} \rrbracket$ is called the twisted adapted $r$-cluster point of type $A_{2 n-1}, D_{n+1}$ or $E_{6}$. A commutation class $\left[\mathscr{Q}^{\prime}\right] \in \llbracket \mathscr{Q} \rrbracket$ is called a twisted adapted class.

(2) For $\sigma$ and $\sigma^{\prime}$ in $(2.1 \mathrm{~d}), \llbracket \mathfrak{Q} \rrbracket:=\llbracket \widetilde{w}_{0}^{\sigma} \rrbracket \sqcup \llbracket \widetilde{w}_{0}^{\sigma^{\prime}} \rrbracket$ is called the triply twisted adapted $r$-cluster point of $D_{4}$. A commutation class $\left[\mathfrak{Q}^{\prime}\right] \in \llbracket \mathfrak{Q} \rrbracket$ is called a triply twisted adapted class.

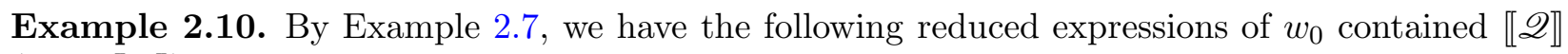

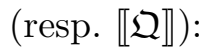

(1) $\widetilde{w}_{0}^{\sigma}:=\left(s_{1} s_{3} s_{4} s_{2} s_{6} s_{5} s_{4} s_{2}\right)^{4} s_{1} s_{3} s_{4} s_{2} \in \llbracket \mathscr{Q} \rrbracket$ of type $E_{6}$;

(2) $\widetilde{w}_{0}^{\sigma}:=\left(s_{2} s_{1} s_{2} s_{3} s_{2} s_{4}\right)^{2} \in \llbracket \mathfrak{Q} \rrbracket$ of type $D_{4}$.

For any quiver $\mathbf{Q}$, we say that a vertex $i$ in $\mathbf{Q}$ is a source (resp. sink) if and only if there are only exiting arrows out of it (resp. entering arrows into it). Let $Q$ be a Dynkin quiver by orienting edges of a Dynkin diagram $\Delta$ of type $A D E$. We denote by $Q^{\text {rev }}$ the quiver obtained by reversing all arrows of $Q$. For any $i \in I$, let $s_{i} Q$ denote the quiver obtained by $Q$ by reversing the arrows incident with $i$. We say that a reduced expression $\widetilde{w}=s_{i_{1}} s_{i_{2}} \cdots s_{i_{\ell(w)}}$ of $w \in W$ is adapted to $Q$ if $i_{k}$ is a sink of the quiver $s_{i_{k-1}} \cdots s_{i_{2}} s_{i_{1}} Q$ for all $1 \leq k \leq \ell(w)$.

The following results are well-known:

Theorem 2.11 ([B9́9, Lus90]).

(1) Any reduced expression $\widetilde{w}_{0}$ of $w_{0}$ is adapted to at most one Dynkin quiver $Q$. 
(2) For each Dynkin quiver $Q$, there is a reduced expression $\widetilde{w}_{0}$ of $w_{0}$ adapted to $Q$. Moreover, any reduced expression $\widetilde{w}_{0}^{\prime}$ in $\left[\widetilde{w}_{0}\right]$ is adapted to $Q$, and the commutation equivalence class $\left[\widetilde{w}_{0}\right]$ is uniquely determined by $Q$. We denote by $[Q]$ of the commutation class $\left[\widetilde{w}_{0}\right]$.

(3) For any Dynkin quivers $Q$ and $Q^{\prime}$ of a fixed Dynkin diagram, the commutation classes $[Q]$ and $\left[Q^{\prime}\right]$ are reflection equivalent.

From Theorem 2.11(3), for a fixed Dynkin diagram $\Delta$, there exists only a single $r$-cluster point $\llbracket \Delta \rrbracket$.

Remark 2.12. In [KO18], the notation $\llbracket Q \rrbracket$ was used instead of $\llbracket \Delta \rrbracket$, where $Q$ is a Dynkin quiver of $\Delta$.

Theorem 2.13 ([OS16a, OS16b]). The number of commutation classes in $\llbracket \Delta \rrbracket$ is the same as the one of $\llbracket \mathscr{Q} \rrbracket$, when their types coincide.

2.3. (Folded) Auslander-Reiten quivers. Note that for any reduced expression $\widetilde{w}_{0}=s_{i_{1}} \cdots s_{i_{\mathrm{N}}}$ of $w_{0}$, we can define a total order $<_{\widetilde{w}_{0}}$ on $\Phi^{+}$as follows:

$$
\alpha_{i_{1}}<_{\widetilde{w}_{0}} s_{i_{1}}\left(\alpha_{i_{2}}\right)<_{\widetilde{w}_{0}} s_{i_{1}} s_{i_{2}}\left(\alpha_{i_{3}}\right)<_{\widetilde{w}_{0}} \cdots<_{\widetilde{w}_{0}} s_{i_{1}} s_{i_{2}} \cdots s_{i_{\mathrm{N}-1}}\left(\alpha_{i_{\mathrm{N}}}\right)
$$

Denote $\beta_{k}^{\widetilde{w}_{0}}:=s_{i_{1}} s_{i_{2}} \cdots s_{i_{k-1}}\left(\alpha_{i_{k}}\right)$. The order $<_{\widetilde{w}_{0}}$ is convex in the following sense: For any $\alpha, \beta \in \Phi^{+}$ satisfying $\alpha+\beta \in \Phi^{+}$, we have either

$$
\alpha<_{\widetilde{w}_{0}} \alpha+\beta<_{\widetilde{w}_{0}} \beta \quad \text { or } \quad \beta<_{\widetilde{w}_{0}} \alpha+\beta<_{\widetilde{w}_{0}} \alpha .
$$

By considering $<_{\widetilde{w}_{0}^{\prime}}$ for all $\widetilde{w}_{0}^{\prime} \in\left[\widetilde{w}_{0}\right]$, we can obtain the convex partial order $\prec_{\left[\widetilde{w}_{0}\right]}$ on $\Phi^{+}$defined as follows:

$$
\left.\alpha \prec \widetilde{w}_{0}\right] \beta \quad \text { if } \alpha<_{\widetilde{w}_{0}^{\prime}} \beta \text { for all } \widetilde{w}_{0}^{\prime} \in\left[\widetilde{w}_{0}\right] .
$$

In [OS15], the combinatorial Auslander-Reiten (AR) quiver $\Upsilon_{\left[\widetilde{w}_{0}\right]}$ is introduced for any commutation class $\left[\widetilde{w}_{0}\right]$ of any finite type.

Algorithm 2.14. Let $\widetilde{w}_{0}=s_{i_{1}} s_{i_{2}} \cdots s_{i_{\mathrm{N}}}$ be a reduced expression of the longest element $w_{0} \in W$. The quiver $\Upsilon_{\widetilde{w}_{0}}=\left(\Upsilon_{\widetilde{w}_{0}}^{0}, \Upsilon_{\widetilde{w}_{0}}^{1}\right)$ associated to $\widetilde{w}_{0}$ is constructed as follows:

(Q1) $\Upsilon_{\widetilde{w}_{0}}^{0}$ consists of $\mathrm{N}$ vertices labeled by $\beta_{1}^{\widetilde{w}_{0}}, \cdots, \beta_{\mathrm{N}}^{\widetilde{w}_{0}}$ (see $\left.(2.4)\right)$.

(Q2) There is an arrow from $\beta_{k}^{\widetilde{w}_{0}}$ to $\beta_{j}^{\widetilde{w}_{0}}$ for $1 \leq j<k \leq \mathrm{N}$ if $(i)$ two vertices $i_{k}$ and $i_{j}$ are connected in the Dynkin diagram, (ii) for $j^{\prime}$ such that $j<j^{\prime}<k$, we have $i_{j^{\prime}} \neq i_{j}, i_{k}$.

Theorem 2.15 ([OS15]).

(1) For any $\widetilde{w}_{0}^{\prime} \sim \widetilde{w}_{0}^{\prime \prime} \in\left[\widetilde{w}_{0}\right]$, we have $\Upsilon_{\widetilde{w}_{0}^{\prime}} \simeq \Upsilon_{\widetilde{w}_{0}^{\prime \prime}}$ as quivers with the same labeling. Thus $\Upsilon_{\left[\widetilde{w}_{0}\right]}$ is well-defined.

(2) $\alpha \prec_{\left[\widetilde{w}_{0}\right]} \beta$ if and only if there exists a path from $\beta$ to $\alpha$ in $\Upsilon_{\left[\widetilde{w}_{0}\right]}$.

(3) When $\left[\widetilde{w}_{0}\right]$ coincides with $[Q]$ for some Dynkin quiver $Q, \Upsilon_{\left[\widetilde{w}_{0}\right]}$ is isomorphic to the AR quiver $\Gamma_{Q}$ associated to the Dynkin quiver $Q$.

We call $\Upsilon_{\left[\widetilde{w}_{0}\right]}$ the twisted Auslander-Reiten $(A R)$ quiver when $\left[\widetilde{w}_{0}\right]$ is (triply) twisted adapted.

By the above theorem, for $\beta \in \Phi^{+}$and $\left[\widetilde{w}_{0}\right]$, we can assign an index $i$ on $\beta$ satisfying $\beta=\beta_{k}^{\widetilde{w}_{0}^{\prime}}$ for some $\widetilde{w}_{0}^{\prime}=\beta_{1}^{\widetilde{w}_{0}}, \cdots, \beta_{\mathrm{N}}^{\widetilde{w}_{0}} \in\left[\widetilde{w}_{0}\right]$ with $i_{k}=i$. We call $i$ the residue with respect to $\left[\widetilde{w}_{0}\right]$.

For the rest of this subsection, we denote by $\left[\widetilde{\mathrm{w}}_{0}\right]$ a reduced expression of $w_{0}$ of type $X$ that is contained in one of $\llbracket \Delta \rrbracket$, $\mathscr{Q} \rrbracket$ or $\llbracket \mathfrak{Q} \rrbracket$, all of which are of simply laced types. Recall that $\left[\widetilde{\mathrm{w}}_{0}\right]$ is 
associated to a Dynkin diagram automorphism $\sigma$. We denote by $\widehat{X}$ the type of the Dynkin diagram obtained by the automorphism $\sigma$. For instance, if $\left[\widetilde{\mathrm{w}}_{0}\right] \in \llbracket \mathscr{Q} \rrbracket$ of type $D_{5}, \widehat{X}$ represents the finite type $C_{4}$. We remark that $X$ is different from $\widehat{X}$ if and only if $\sigma \neq \mathrm{id}$.

Definition 2.16. Let a be an arrow between a vertex of residue $i$ and a vertex of residue $j$ in $\Upsilon_{\left[\widetilde{w}_{0}\right]}$. Recall that $\widehat{I}$ is the index set of the Dynkin diagram $\widehat{\Delta}$ of $(2.1)$. Let $\left\{\widehat{\alpha}_{\widehat{\imath}} \mid \widehat{\imath} \in \widehat{I}\right\}$ be the corresponding simple roots of $\widehat{\Delta}$. We define the length $\ell($ a) as follows:

$$
\ell(\mathrm{a}):=\min \left\{\left|\widehat{\alpha}_{\widehat{\imath}}\right|^{2},\left|\widehat{\alpha}_{\widehat{\jmath}}\right|^{2}\right\}
$$

Let $\mathrm{d}$ denote the order of the Dynkin diagram automorphism $\sigma$.

Furthermore, the lengths of arrows in a (twisted) AR quiver $\Upsilon_{\left[\widetilde{w}_{0}\right]}$ is equal to $1 / \mathrm{d}$ except for possibly when $\sigma$ is $(2.1 \mathrm{a})$ or $(2.1 \mathrm{c})$, in which case the length of an arrow may also be 1 .

Then it is proved in [OS16a, OS16b] that we can assign $\beta \in \Upsilon_{\left[\widetilde{w}_{0}\right]}$ a coordinate $\Omega_{\left[\widetilde{w}_{0}\right]}(\beta):=(i, p) \in$ $I \times \frac{1}{\mathrm{~d}} \mathbb{Z}$ which is compatible to the lengths of arrows. Here $i$ is the residue of $\beta$ with respect to $\left[\widetilde{\mathrm{w}}_{0}\right]$. Here we give examples instead of giving all the details (see [OS16a, OS16b] for details). We remark here that the coordinate system on $\Gamma_{Q}$ was originally given in [HL10].

\section{Example 2.17.}

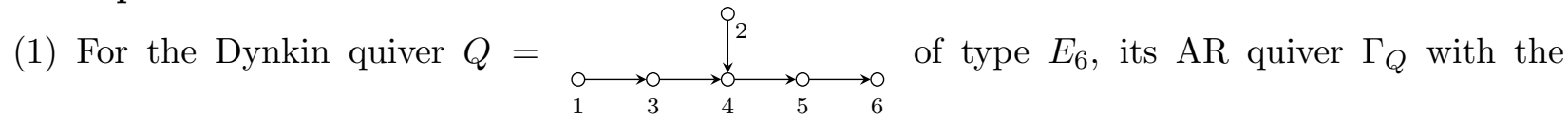
coordinate system can be depicted as follows: $\left(\left(\begin{array}{l}a_{1} a_{2} a_{3} \\ a_{4} a_{5} a_{6}\end{array}\right):=\sum_{i=1}^{6} a_{i} \alpha_{i}\right)$

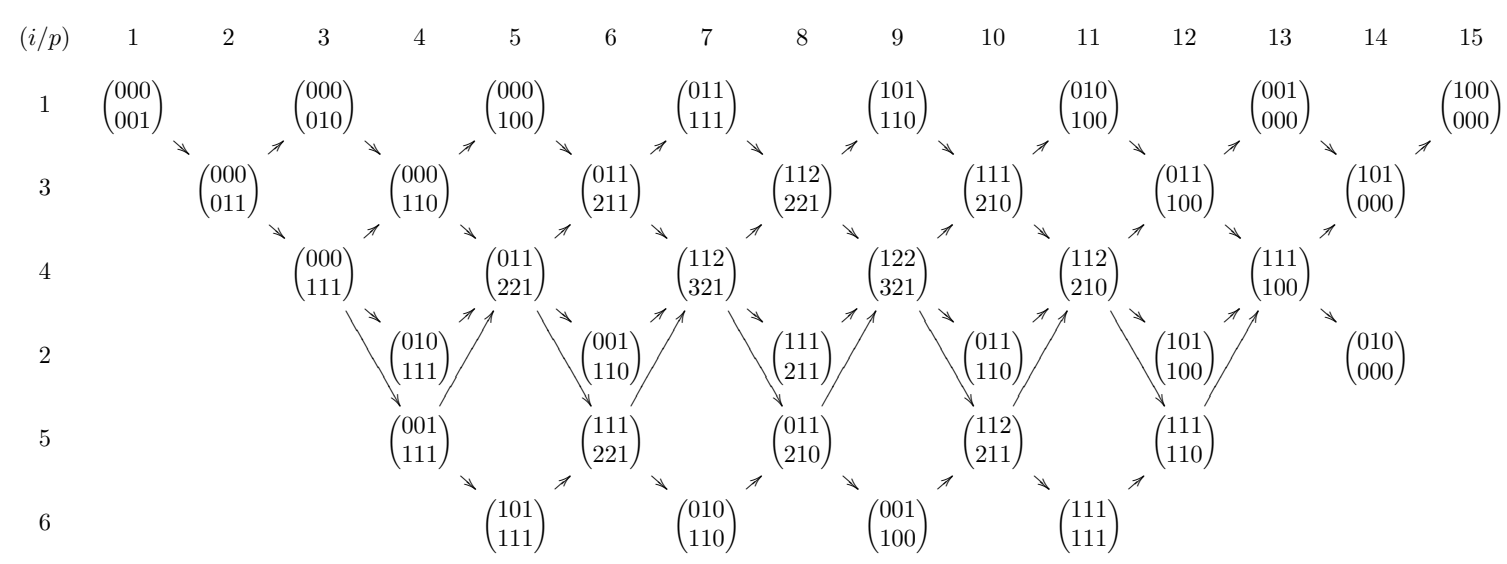


(2) For $\left[\widetilde{\mathrm{w}}_{0}\right]=[\mathscr{Q}]$ of type $E_{6}$ in (1) of Example 2.10, its twisted AR quiver $\Upsilon_{[\mathscr{Q}]}$ with the coordinate system can be depicted as follows:

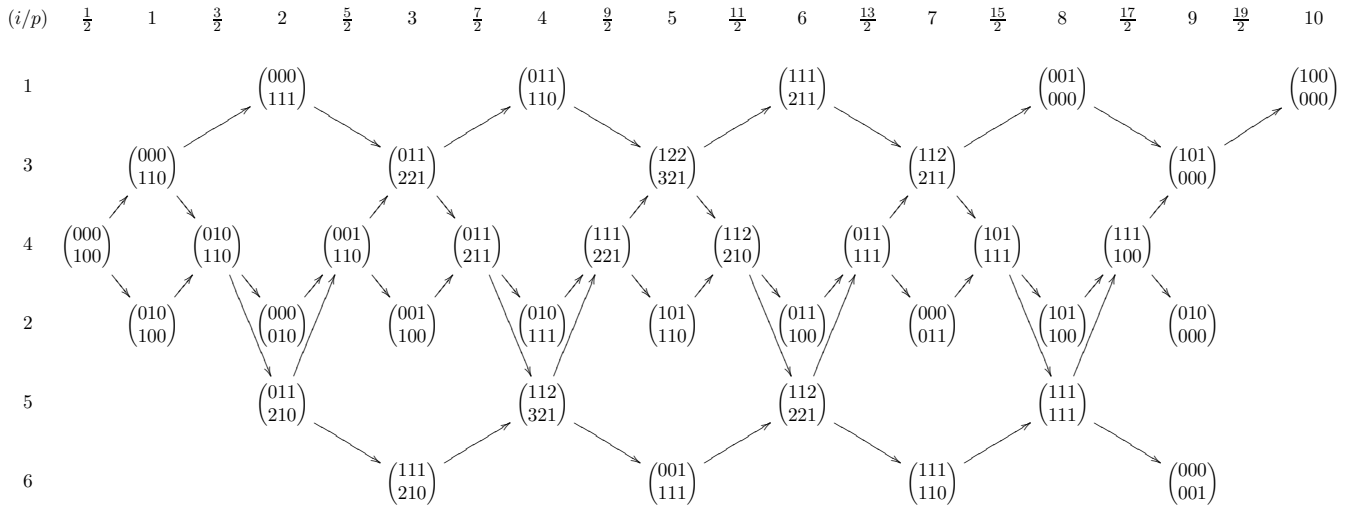

\section{Example 2.18.}

(1) For the Dynkin quiver $Q=\underset{1}{\mathrm{O}} 3$ system can be depicted as follows:

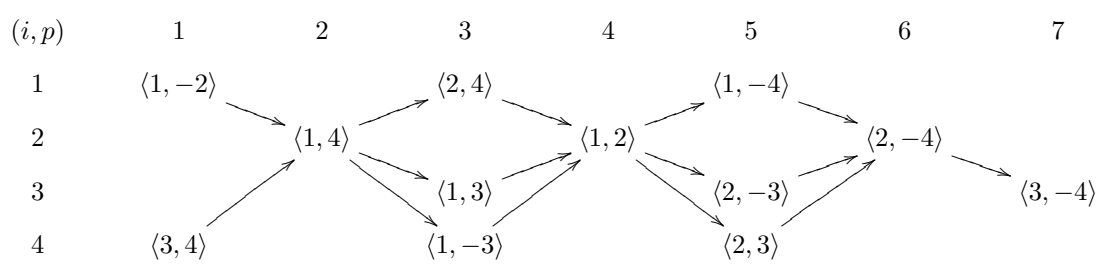

Here $\langle a, \pm b\rangle:=\epsilon_{a} \pm \epsilon_{b}$.

(2) For $\left[\widetilde{\mathrm{w}}_{0}\right]=[\mathfrak{Q}]$ of type $D_{4}$ in $(2)$ of Example 2.10, its twisted AR quiver $\Upsilon_{[\mathfrak{Q}]}$ with the coordinate system can be depicted as follows:

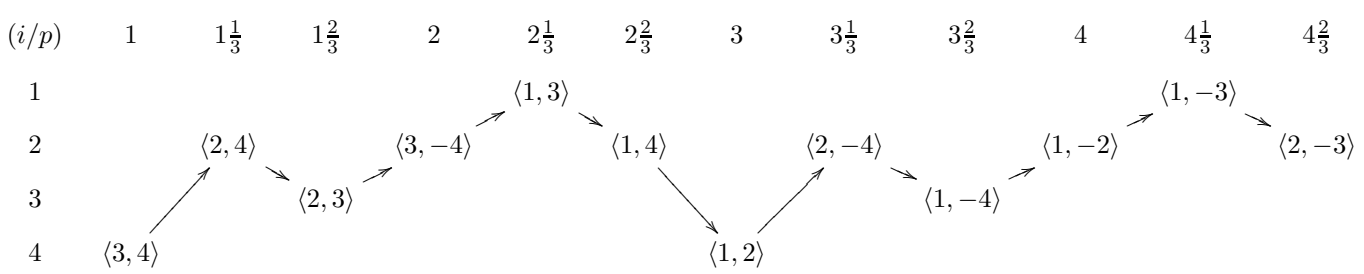

If $\sigma \neq \mathrm{id}$, we can construct the folded $A R$ quiver $\widehat{\Upsilon}_{\left[\widetilde{\mathrm{w}}_{0}\right]}$ from $\Upsilon_{\left[\widetilde{\mathrm{w}}_{0}\right]}$ via the involution $\sigma$ in the following sense: There are no vertices $\alpha$ and $\beta$ in $\Upsilon_{[\mathscr{Q}]}\left(\right.$ resp. $\left.\Upsilon_{[\mathfrak{Q}]}\right)$ such that

$$
(\widehat{\imath}, p)=\left(\widehat{\jmath}, p^{\prime}\right) \text {, where } \Omega_{\left[\widetilde{\mathrm{w}}_{0}\right]}(\alpha)=(i, p) \text { and } \Omega_{\left[\widetilde{\mathrm{w}}_{0}\right]}(\beta)=\left(j, p^{\prime}\right)\left(\left[\widetilde{\mathrm{w}}_{0}\right]=[\mathscr{Q}] \text { or }[\mathfrak{Q}]\right) \text {. }
$$

Thus we can assign $\beta \in \widehat{\Upsilon}_{\left[\widetilde{\mathrm{w}}_{0}\right]}$ a $\widehat{\Omega}_{\left[\widetilde{\mathrm{w}}_{0}\right]}(\beta):=(\widehat{\imath}, p) \in \widehat{I} \times \frac{1}{\mathrm{~d}} \mathbb{Z}$, called the folded coordinate of $\beta$. 
Example 2.19. In Example 2.17, $\Upsilon_{[\mathscr{Q}]}$ can be folded into $\widehat{\Upsilon}_{[\mathscr{Q}]}$ as follows:

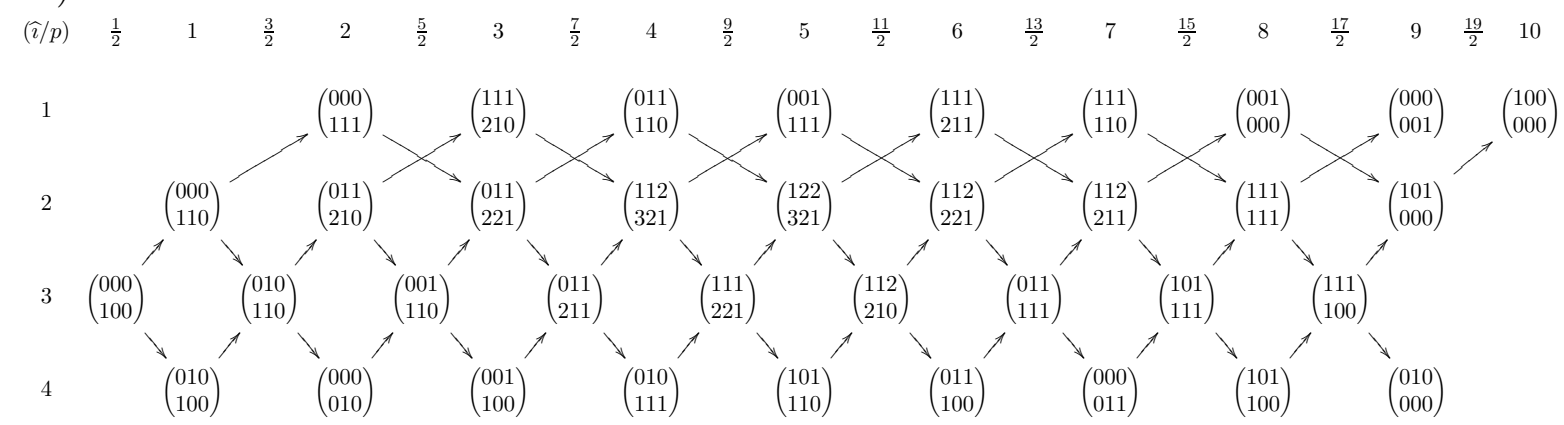

Example 2.20. In Example 2.18, $\Upsilon_{[\mathfrak{Q}]}$ can be folded into $\widehat{\Upsilon}_{[\mathfrak{Q}]}$ as follows:

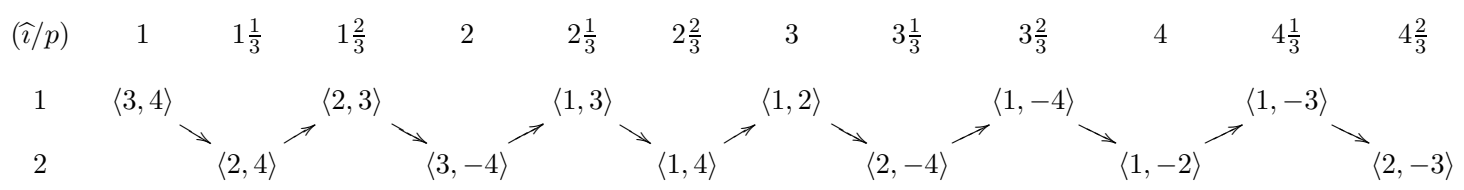

For simplicity, we also use $\widehat{\Upsilon}_{\left[\widetilde{\mathrm{w}}_{0}\right]}$ when $\sigma=\mathrm{id}$; in that case, $\left[\widetilde{\mathrm{w}}_{0}\right]=[Q]$ for some Dynkin quiver $Q$, $\widehat{X}=X$ and $\widehat{\Upsilon}_{\left[\widetilde{\mathrm{w}}_{0}\right]}=\Gamma_{Q}$.

2.4. Statistics on $\widehat{\Upsilon}_{\left[\widetilde{w}_{0}\right]}$. Recall the notation $\beta_{k}^{\widetilde{w}_{0}}$ and the total order $<_{\widetilde{w}_{0}}$ for $\widetilde{w}_{0}$. Consider a sequence $\underline{m}=\left(\underline{m}_{1}, \underline{m}_{2}, \ldots, \underline{m}_{\mathrm{N}}\right) \in \mathbb{Z}_{\geq 0}^{\mathrm{N}}$, and we define $\mathrm{wt}_{\widetilde{w}_{0}}(\underline{m})=\sum_{i=1}^{\mathrm{N}} \underline{m}_{i} \beta_{i}^{\widetilde{w}_{0}} \in \mathrm{Q}^{+}$.

Definition 2.21 ([McN15, Oh19]). We define the partial orders $<_{\widetilde{w}_{0}}^{\mathrm{b}}$ and $\prec_{\left[\widetilde{w}_{0}\right]}^{\mathrm{b}}$ on $\mathbb{Z}_{\geq 0}^{\mathrm{N}}$ as follows:

(i) $<\widetilde{w}_{0}^{\mathrm{b}}$ is the bi-lexicographical partial order induced by $<\widetilde{w}_{0}$. Namely, $\underline{m}<{\widetilde{w_{0}}}_{\underline{m}^{\prime}}^{\mathrm{b}}$ if there exist $j$ and $k(1 \leq j \leq k \leq \mathrm{N})$ such that

- $\underline{m}_{s}=\underline{m}_{s}^{\prime}$ for $1 \leq s<j$ and $\underline{m}_{j}<\underline{m}_{j}^{\prime}$,

- $\underline{m}_{s}=\underline{m}_{s}^{\prime}$ for $k<s \leq \mathrm{N}$ and $\underline{m}_{k}<\underline{m}_{k}^{\prime}$.

(ii) For sequences $\underline{m}$ and $\underline{m}^{\prime}$, we have $\underline{m} \prec_{\left[\widetilde{w}_{0}\right]}^{\mathrm{b}} \underline{m}^{\prime}$ if and only if $\operatorname{wt}_{\widetilde{w}_{0}}(\underline{m})=\mathrm{wt}_{\widetilde{w}_{0}}\left(\underline{m}^{\prime}\right)$ and $\underline{n}<_{\widetilde{w}_{0}^{\prime}} \underline{n}^{\prime}$ for all $\widetilde{w}_{0}^{\prime} \in\left[\widetilde{w}_{0}\right]$, where $\underline{n}$ and $\underline{n}^{\prime}$ are sequences such that $\underline{n}_{\widetilde{w}_{0}^{\prime}}=\underline{m}_{\widetilde{w}_{0}}$ and $\underline{n}_{\widetilde{w}_{0}^{\prime}}^{\prime}=\underline{m}_{\widetilde{w}_{0}}$.

We give the following definitions from [McN15, Oh19] but instead using more of the language of posets. We say a sequence $\underline{m}=\left(\underline{m}_{1}, \underline{m}_{2}, \ldots, \underline{m}_{\mathrm{N}}\right) \in \mathbb{Z}_{\geq 0}^{\mathrm{N}}$ is $\left[\widetilde{w}_{0}\right]$-simple if it is minimal with respect to the partial order $\prec_{\left[\widetilde{w}_{0}\right]}^{\mathrm{b}}$. For a given $\left[\widetilde{w}_{0}\right]$-simple sequence $\underline{s}=\left(s_{1}, \ldots, s_{\mathrm{N}}\right) \in \mathbb{Z}_{\geq 0}^{\mathrm{N}}$, we call a cover ${ }^{1}$ of $\underline{s}$ under $\prec_{\left[\widetilde{w}_{0}\right]}^{\mathrm{b}}$ a $\left[\widetilde{w}_{0}\right]$-minimal sequence of $\underline{s}$. The $\left[\widetilde{w}_{0}\right]$-distance of a sequence $\underline{m}$ is the largest integer $k \geq 0$ such that

and $\underline{m}^{(0)}$ is $\left[\widetilde{w}_{0}\right]$-simple.

$$
\underline{m}^{(0)} \prec_{\left[\widetilde{w}_{0}\right]}^{\mathrm{b}} \cdots \prec_{\left[\widetilde{w}_{0}\right]}^{\mathrm{b}} \underline{m}^{(k)}=\underline{m}
$$

We call a sequence $\underline{m}$ a pair if $|\underline{m}|:=\sum_{i=1}^{\mathrm{N}} m_{i}=2$ and $m_{i} \leq 1$ for $1 \leq i \leq \mathrm{N}$. We mainly use the notation $p$ for a pair. Consider a pair $p$ such that there exists a unique $\left[\widetilde{w}_{0}\right]$-simple sequence $\underline{s}$ satisfying $\underline{s} \preceq_{\left[\widetilde{w}_{0}\right]}^{\underline{b}} \underline{p}$, we call $\underline{s}$ the $\left[\widetilde{w}_{0}\right]$-socle of $\underline{p}$ and denoted it by $\operatorname{soc}_{\left[\widetilde{w}_{0}\right]}(\underline{p})$.

\footnotetext{
${ }^{1}$ Recall that a cover of $x$ in a poset $P$ with partial order $\prec$ is an element $y \in P$ such that $x \prec y$ and there does not exists $y^{\prime} \in P$ such that $x \prec y^{\prime} \prec y$.
} 
Proposition 2.22 ([BKM14, Lemma 2.6]). For $\gamma \in \Phi^{+} \backslash \Pi$ and any $\widetilde{w}_{0}$ of $w_{0}$, a $\left[\widetilde{w}_{0}\right]$-minimal sequence of $\gamma$ is indeed a pair $(\alpha, \beta)$ for some $\alpha, \beta \in \Phi^{+}$such that $\alpha+\beta=\gamma$.

As we did in the previous subsection, $\left[\widetilde{\mathrm{w}}_{0}\right]$ denotes a commutation class in $\llbracket \Delta \rrbracket$, $\llbracket \mathscr{Q} \rrbracket$ or $\llbracket \mathfrak{Q} \rrbracket$. Recall, for each $\left[\widetilde{\mathrm{w}}_{0}\right]$, we can correspond $\widehat{X}, \sigma, \widehat{I}$ and d.

Following [Oh19, OS16a, OS16b], for a folded AR quiver $\widehat{\Upsilon}_{\left[\widetilde{w}_{0}\right]}$, indices $\widehat{k}, \widehat{l} \in \widehat{I}$ and an integer $t \in \mathbb{Z}_{\geq 1}$, we define the subset $\Phi_{\left[\widetilde{w}_{0}\right]}(\widehat{k}, \widehat{l})[t] \subset \Phi^{+} \times \Phi^{+}$as the pairs $(\alpha, \beta) \in \Phi^{+} \times \Phi^{+}$such that $\alpha$ and $\beta$ are comparable under $\prec_{\left[\widetilde{w}_{0}\right]}$ and

$$
\left\{\widehat{\Omega}_{\left[\widetilde{\mathrm{w}}_{0}\right]}(\alpha), \widehat{\Omega}_{\left[\widetilde{\mathrm{w}}_{0}\right]}(\beta)\right\}=\{(\widehat{k}, a),(\widehat{l}, b)\} \quad \text { such that } \quad|a-b|=\frac{t}{\mathrm{~d}} .
$$

Proposition 2.23 ([Oh19, OS16a, OS16b]).

(1) For any $(\alpha, \beta),\left(\alpha^{\prime}, \beta^{\prime}\right) \in \Phi_{\left[\widetilde{w}_{0}\right]}(\widehat{k}, \widehat{l})[t]$, we have

$$
\operatorname{dist}_{\left[\widetilde{\mathrm{w}}_{0}\right]}(\alpha, \beta)=\operatorname{dist}_{\left[\widetilde{\mathrm{w}}_{0}\right]}\left(\alpha^{\prime}, \beta^{\prime}\right) .
$$

Moreover, we denote by $o_{t}^{\left[\widetilde{\mathrm{w}}_{0}\right]}(\widehat{k}, \widehat{l}):=\operatorname{dist}_{\left[\widetilde{\mathrm{w}}_{0}\right]}(\alpha, \beta)$ for any $(\alpha, \beta) \in \Phi_{\left[\widetilde{\mathrm{w}}_{0}\right]}(\widehat{k}, \widehat{l})[t]$.

(2) The integer, defined by

$$
\theta_{t}^{\left[\widetilde{\mathrm{w}}_{0}\right]}(\widehat{k}, \widehat{l})= \begin{cases}\max \left\{o_{t}^{[Q]}(\widehat{k}, \widehat{l}), o_{t}^{\left[Q^{\mathrm{rev}}\right]}(\widehat{k}, \widehat{l})\right\} & \text { if }\left[\widetilde{\mathrm{w}}_{0}\right]=[Q] \text { for some } Q, \\ \left\lceil o_{t}^{\left[\widetilde{\mathrm{w}}_{0}\right]}(\widehat{k}, \widehat{l}) / \mathrm{d}\right] & \text { otherwise }\end{cases}
$$

does not depend on the choice of $\left[\widetilde{\mathrm{w}}_{0}^{\prime}\right],\left[\widetilde{\mathrm{w}}_{0}^{\prime \prime}\right] \in \llbracket \widetilde{\mathrm{w}}_{0} \rrbracket$.

From Proposition 2.23, we can define the (folded) distance polynomial $D_{\widehat{k}, \widehat{l}}^{\widehat{X}}(z ;-q)$ for $\widehat{k}, \widehat{l} \in \widehat{I}$ on $\llbracket \widetilde{\mathrm{w}}_{0} \rrbracket$ as

$$
D_{\widehat{k}, \widehat{l}}^{\llbracket \widetilde{\mathrm{w}}_{0} \rrbracket}(z ;-q)=\prod_{t \in \mathbb{Z}_{\geq 0}}\left(z-(-1)^{\kappa} q^{t}\right)^{\theta_{t}^{\llbracket \widetilde{\mathrm{w}}_{0} \rrbracket}(\widehat{k}, \widehat{l})},
$$

where

$$
\kappa= \begin{cases}\widehat{k}+\widehat{l} & \text { if } \sigma \text { is from }(2.1 \mathrm{a}) \text { or }(2.1 \mathrm{c}) \\ t & \text { otherwise }\end{cases}
$$

\section{Quantum AFFine Algebras}

3.1. Quantum affine algebra. In this section, we review the representation theory of finite dimensional integrable modules over quantum affine algebras referring [AK97, Kas02]. When we deal with quantum affine algebras, we take a base field $\mathbf{k}$ which is the algebraic closure of $\mathbb{C}(q)$ in $\cup_{m>0} \mathbb{C}\left(\left(q^{1 / m}\right)\right)$.

Let $A$ be a generalized Cartan matrix of affine type. We choose $0 \in I$ as the leftmost vertex in the table [Kac90, page 54,55] except in the $A_{2 n}^{(2)}$ case, in which case we take the longest simple root as $\alpha_{0}$. For the exceptional types, we follow the labeling given in Figure 1, and otherwise we follow the labeling of [Kac90]. We denote the imaginary root by $\delta=\sum_{i \in I} d_{i} \alpha_{i}$ and the canonical central element $c=\sum_{i \in I} c_{i} h_{i}[\operatorname{Kac} 90$, Chapter 4$]$. We normalize the bilinear form $(\cdot, \cdot)$ by

$$
(\delta, \lambda)=\langle c, \lambda\rangle \quad \text { for any } \lambda \in P .
$$




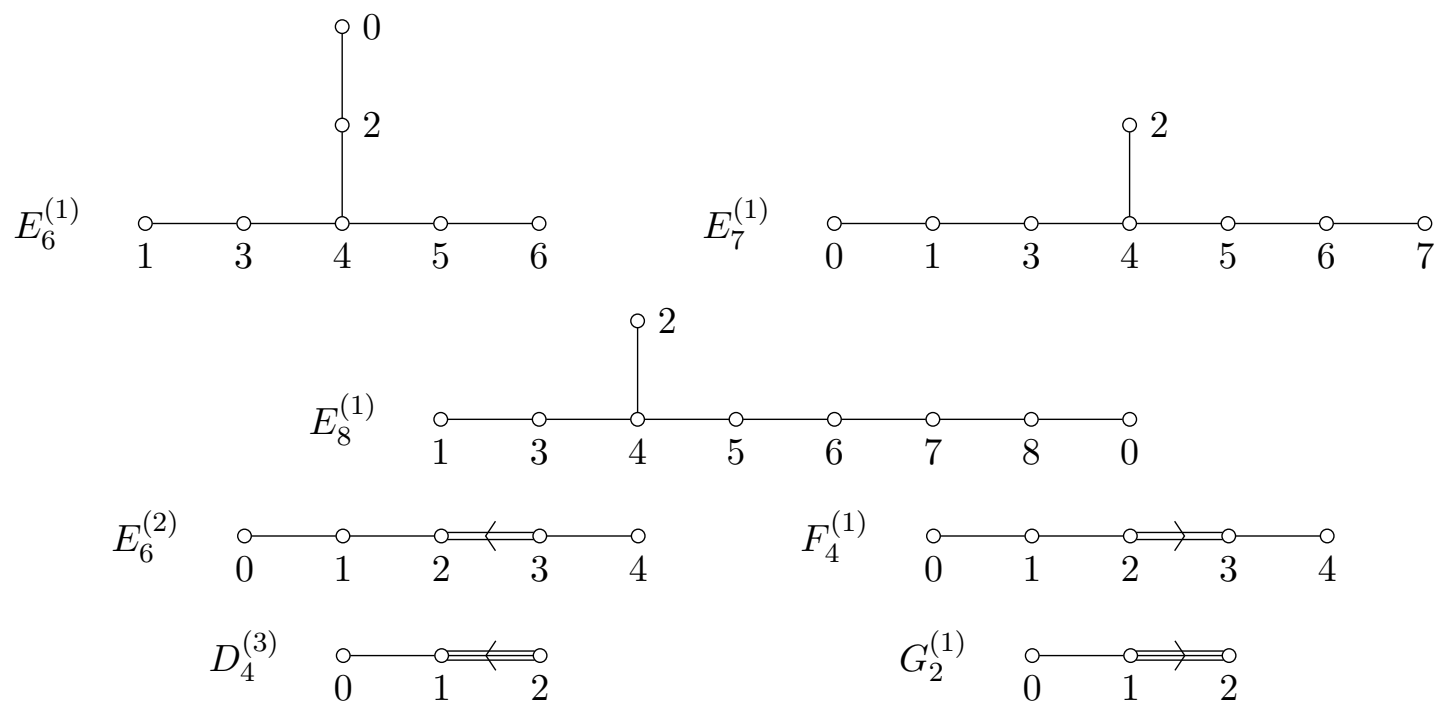

Figure 1. Dynkin diagrams for the exceptional affine types.

For the affine Cartan datum $\left(\mathrm{A}, P, \Pi, P^{\vee}, \Pi^{\vee},(\cdot, \cdot)\right)$ and associated quantum group $U_{q}(\mathfrak{g})$, let $U_{q}^{\prime}(\mathfrak{g})$ denote the quantum affine algebra that is the subalgebra of $U_{q}(\mathfrak{g})$ generated by $e_{i}, f_{i}, K_{i}^{ \pm 1}$ $(i \in I) .^{2}$ Let us denote by $\operatorname{Mod}\left(U_{q}^{\prime}(\mathfrak{g})\right)$ the category of left $U_{q}^{\prime}(\mathfrak{g})$-modules.

Let $U_{q}\left(\mathfrak{g}_{0}\right)$ denote the quantum group of the corresponding finite-type given by $I_{0}:=I \backslash\{0\}$, and note that $U_{q}\left(\mathfrak{g}_{0}\right) \subset U_{q}(\mathfrak{g})$ as the subalgebra generated by $e_{i}, f_{i}, K_{i}$ for $i \in I_{0}$. Let $\bar{\Lambda}_{i}$ denote the fundamental weights associated to the Cartan datum of $\mathfrak{g}_{0}$.

Let us denote by ${ }^{-}$the involution of $U_{q}^{\prime}(\mathfrak{g})$ defined as follows:

$$
e_{i} \mapsto e_{i}, \quad f_{i} \mapsto f_{i}, \quad K_{i} \mapsto K_{i}, \quad q^{1 / \gamma} \rightarrow q^{-1 / \gamma} .
$$

Set $P_{\mathrm{cl}}:=P / \mathbb{Z} \delta$ and $\mathrm{cl}: P \rightarrow P_{\mathrm{cl}}$ as the canonical projection. Then $P_{\mathrm{cl}}=\bigoplus_{i \in I} \mathbb{Z} \mathrm{cl}\left(\Lambda_{i}\right)$. We also define the subset $P_{\mathrm{cl}}^{0}$ of $P_{\mathrm{cl}}$ as follows:

$$
P_{\mathrm{cl}}^{0}:=\left\{\lambda \in P_{\mathrm{cl}} \mid\langle c, \lambda\rangle=0\right\} \subset P_{\mathrm{cl}} .
$$

We denote by $W_{\text {aff }}$ the affine Weyl group generated by $s_{i} \in \operatorname{Aut}(P)(i \in I)$, where $s_{i}(\mu)=$ $\mu-\left\langle h_{i}, \mu\right\rangle \alpha_{i}$. Then $W_{\text {aff }}$ acts also on $P_{\mathrm{cl}}$ and $P_{\mathrm{cl}}^{0}$.

We call a $U_{q}^{\prime}(\mathfrak{g})$-module $M$ integrable if

(a) $M$ admits a weight space decomposition

$$
M=\bigoplus_{\mu \in P_{\mathrm{cl}}} M_{\mu}, \quad \text { where } M_{\mu}=\left\{u \in M \mid K_{i} u=q_{i}^{\left\langle h_{i}, \mu\right\rangle} \text { for all } i \in I\right\},
$$

(b) the actions of $e_{i}$ and $f_{i}$ are locally nilpotent for all $i \in I$.

We denote by $\mathcal{C}_{\mathfrak{g}}$ the abelian tensor category consisting of finite dimensional integrable $U_{q}^{\prime}(\mathfrak{g})$ modules. For a simple module $M$ in $\mathcal{C}_{\mathfrak{g}}$, there exists a non-zero vector $v_{\lambda} \in M$ of weight $\lambda \in P_{\mathrm{cl}}^{0}$

\footnotetext{
${ }^{2}$ The quantum group $U_{q}^{\prime}(\mathfrak{g})$ also may be considered as the quantum group $U_{q}\left(\mathfrak{g}^{\prime}\right)$ associated to the derived subalgebra $\mathfrak{g}^{\prime}:=[\mathfrak{g}, \mathfrak{g}]$.
} 
such that $\left\langle h_{i}, \lambda\right\rangle \geq 0$ for all $i \in I_{0}$ and $\mathbf{k} v=M_{\lambda}$. Such a weight $\lambda$ is called the dominant extremal weight of $M$ and a non-zero vector in $M_{\lambda}$ is called a dominant extremal vector of $M$.

For an integrable $U_{q}^{\prime}(\mathfrak{g})$-module $M$, the affinization $M_{\mathrm{aff}}:=\mathbf{k}\left[z, z^{-1}\right] \otimes M$ of $M$ is considered as a vector space over $\mathbf{k}$ and is equipped with a $U_{q}^{\prime}(\mathfrak{g})$-module structure

$$
e_{i}\left(u_{z}\right)=z^{\delta_{i, 0}}\left(e_{i} u\right)_{z}, \quad f_{i}\left(u_{z}\right)=z^{-\delta_{i, 0}}\left(f_{i} u\right)_{z}, \quad K_{i}\left(u_{z}\right)=\left(K_{i} u\right)_{z},
$$

for all $i \in I$. Here $u_{z}$ denotes $\mathbf{1} \otimes u \in M_{\text {aff }}$ for $u \in M$. We denote by the action of $z$ on $M_{\text {aff }}$ by $z_{M}$ and sometimes write $M_{z}$ instead of $M_{\text {aff }}$.

For $a \in \mathbf{k}^{\times}$, we define the evaluation module of $M$ at $a$ as

$$
M_{a}:=M_{z} /(z-a) M_{z}
$$

and call the $a$ the spectral parameter. Note that for $U_{q}^{\prime}(\mathfrak{g})$-modules $M$ and $N$, we have

$$
(M \otimes N)_{a} \simeq M_{a} \otimes N_{a} .
$$

For a $U_{q}^{\prime}(\mathfrak{g})$-module $M$, we denote by the $U_{q}^{\prime}(\mathfrak{g})$-module $\bar{M}=\{\bar{u} \mid u \in M\}$ whose module structure is give as follows:

$$
x \bar{u}:=\overline{\bar{x}} u,
$$

where $x \in U_{q}^{\prime}(\mathfrak{g})$. Then we have

$$
\overline{M_{a}} \simeq \bar{M}_{\bar{a}}, \quad \overline{M \otimes N} \simeq \bar{N} \otimes \bar{M} .
$$

For each $i \in I_{0}$, we set

$$
\varpi_{i}:=\operatorname{gcd}\left(c_{0}, c_{i}\right)^{-1} \operatorname{cl}\left(c_{0} \Lambda_{i}-c_{i} \Lambda_{0}\right) \in P_{\mathrm{cl}}^{0},
$$

which is called an $i$-th level 0 fundamental weight. Then $\left\{\varpi_{i}\right\}_{i \in I_{0}}$ is a basis of $P_{\mathrm{cl}}^{0}$. Therefore, for each $i \in I_{0}$, there exists a unique simple $U_{q}^{\prime}(\mathfrak{g})$-module $V\left(\varpi_{i}\right) \in \mathcal{C}_{\mathfrak{g}}$ whose dominant extremal weight is $\varpi_{i}$ and satisfies the following properties:

We can take $u_{\mu} \in V\left(\varpi_{i}\right)_{\mu}$ for each $\mu \in W \varpi_{i} \subset P_{\text {cl }}$ such that

(a) for $j \in I$ and $\mu \in W \varpi_{i}$ such that $\left\langle h_{i}, \mu\right\rangle \geq 0, e_{j} u_{\mu}=0$ and $f_{j}^{\left(\left\langle h_{i}, \mu\right\rangle\right)} u_{\mu}=u_{s_{j} \mu}$,

(b) for $j \in I$ and $\mu \in W \varpi_{i}$ such that $\left\langle h_{i}, \mu\right\rangle<0, f_{j} u_{\mu}=0$ and $e_{j}^{\left(-\left\langle h_{i}, \mu\right\rangle\right)} u_{\mu}=u_{s_{j} \mu}$,

(c) $M$ is generated by $u_{\varpi_{i}}$.

We call $V\left(\varpi_{i}\right)$ the fundamental representation.

We say that a simple $U_{q}^{\prime}(\mathfrak{g})$-module $M$ is good if it has a bar involution, a crystal basis with simple crystal graph and a lower global basis (see [Kas02, §8] for precise definitions). Note that the fundamental representation $V\left(\varpi_{i}\right)_{x}$ is good. For each $M \in \mathcal{C}_{g}$, there exist the right dual ${ }^{*} M$ and the left dual $M^{*}$ of $M$ in the following sense: we have $U_{q}^{\prime}(\mathfrak{g})$-module isomorphisms

$$
\begin{aligned}
& \operatorname{Hom}(M \otimes X, Y) \simeq \operatorname{Hom}\left(X,{ }^{*} M \otimes Y\right), \operatorname{Hom}\left(X \otimes{ }^{*} M, Y\right) \simeq \operatorname{Hom}(X, Y \otimes M), \\
& \operatorname{Hom}\left(M^{*} \otimes X, Y\right) \simeq \operatorname{Hom}(X, M \otimes Y), \operatorname{Hom}(X \otimes M, Y) \simeq \operatorname{Hom}\left(X, Y \otimes M^{*}\right),
\end{aligned}
$$

which are functorial in $U_{q}^{\prime}(\mathfrak{g})$-modules $X$ and $Y$. The duals of a fundamental representation $V\left(\varpi_{i}\right)$ $\left(i \in I_{0}\right)$ are given as follows:

$$
{ }^{*} V\left(\varpi_{i}\right) \simeq V\left(\varpi_{i^{*}}\right)_{p^{*}} \text { and } V\left(\varpi_{i}\right)^{*} \simeq V\left(\varpi_{i^{*}}\right)_{\left(p^{*}\right)^{-1}}
$$


where $p^{*}:=(-1)^{\left\langle\rho^{\vee}, \delta\right\rangle} q^{\langle c, \rho\rangle}$. Here $\rho$ (resp. $\rho^{\vee}$ ) denotes an element in $P$ (resp. $P^{\vee}$ ) satisfying $\left\langle h_{i}, \rho\right\rangle=1$ (resp. $\left\langle\rho^{\vee}, \alpha_{i}\right\rangle=1$ ) for all $i \in I$, and $i^{*}$ denotes the index in $I$ determined by $w_{0}\left(\alpha_{i}\right)=-\alpha_{i^{*}}$, where $w_{0}$ is the longest element of $W_{0}=\left\langle s_{i} \mid i \in I_{0}\right\rangle$.

For the fundamental representations, we can compute the $U_{q}\left(\mathfrak{g}_{0}\right)$-decomposition by using the (virtual) Kleber algorithm [Kle98, OSS03].

Remark 3.1. When we say a vector $v$ is the highest weight vector of an irreducible $V(\lambda)$ component in the $U_{q}\left(\mathfrak{g}_{0}\right)$-decomposition of a $U_{q}^{\prime}(\mathfrak{g})$-module, we will always mean "up to scalar in $\mathbf{k} . "$

3.1.1. Module structure for affine minuscule representations. Let $\mathfrak{g}$ be dual to an untwisted affine type. Suppose $r \in I_{0}$ is in the orbit of 0 under some affine Dynkin diagram automorphism. Then we say $V\left(\varpi_{r}\right)$ is a affine minuscule representation since $V\left(\varpi_{r}\right) \simeq V\left(\bar{\Lambda}_{r}\right)$ as $U_{q}\left(\mathfrak{g}_{0}\right)$-modules and $V\left(\bar{\Lambda}_{r}\right)$ is a minuscule representation of $U_{q}\left(\mathfrak{g}_{0}\right)$.

We consider the crystal $B\left(\varpi_{r}\right)$ of the fundamental representation $V\left(\varpi_{r}\right)$ [Kas02, NS03, NS06a]. Denote on $B\left(\varpi_{r}\right)$ the Kashiwara operators [Kas90, Kas91] by $\widetilde{e}_{i}, \widetilde{f}_{i}: B\left(\varpi_{r}\right) \rightarrow B\left(\varpi_{r}\right) \sqcup\{\mathbf{0}\}$ and the weight function by wt: $B\left(\varpi_{r}\right) \rightarrow P_{\mathrm{cl}}$. We define a $U_{q}(\mathfrak{g})$-module $\mathbb{V}\left(\varpi_{r}\right):=\mathbf{k}\left\{v_{b} \mid b \in B\left(\varpi_{r}\right)\right\}$ by

$$
e_{i} v_{b}=v_{\widetilde{e}_{i} b}, \quad f_{i} v_{b}=v_{\widetilde{f}_{i} b}, \quad K_{i} v_{b}=q_{i}^{\left\langle h_{i}, \operatorname{wt}(b)\right\rangle} v_{b},
$$

where we consider $v_{\mathbf{0}}:=0$.

Proposition 3.2. $\mathbb{V}\left(\varpi_{r}\right)$ is a $U_{q}^{\prime}(\mathfrak{g})$-module.

Proof. Note that since $V\left(\bar{\Lambda}_{i}\right)$ is a minuscule representation, so from, e.g., [Ste01], as we can parameterize the weight spaces in $V\left(\varpi_{r}\right)$ by the minimal length coset representatives of $W_{0} / \operatorname{Stab}_{W_{0}}\left(\bar{\Lambda}_{r}\right)$, recalling that the stabilizer of $\bar{\Lambda}_{r}$ in $W_{0}$ is a parabolic subgroup of $W_{0}$. Hence, we have $\widetilde{e}_{i}^{2} b=\widetilde{f}_{i}^{2} b=0$ for all $b \in B\left(\varpi_{r}\right)$ and $i \in I$. Therefore, from [Ste01, Prop. 2.1], we have that $\mathbb{V}\left(\bar{\Lambda}_{r}\right)$ is a $U_{q}^{\prime}(\mathfrak{g})$ module.

Proposition 3.3. We have $\mathbb{V}\left(\varpi_{r}\right) \simeq V\left(\varpi_{r}\right)$.

Proof. Let $u_{\varpi_{r}} \in B\left(\varpi_{r}\right)$ be the unique element such that $e_{i} u_{\varpi_{r}}=0$ for all $i \in I_{0}$. For any vector

$$
v=\sum_{b \in B\left(\varpi_{r}\right)} \xi_{b} v_{b} \in \mathbb{V}\left(\varpi_{r}\right)
$$

choose a maximal length sequence $\left(i_{j} \in I_{0}\right)_{j=1}^{\ell}$ such that there exists $b \in B\left(\varpi_{i}\right)$ that satisfies $\widetilde{e}_{i_{1}} \cdots \widetilde{e}_{i_{\ell}} b=u_{\bar{\Lambda}_{r}}$ and $\xi_{b} \neq 0$. Therefore, we have

$$
e_{i_{1}} \cdots e_{i_{\ell}} v=\xi_{b} v_{u_{\varpi_{r}}}
$$

and hence $\mathbb{V}\left(\varpi_{r}\right)$ is simple. Since $\mathbb{V}\left(\varpi_{r}\right)$ has a dominant extremal vector of weight $\varpi_{r}$, the claim follows.

From Proposition 3.3, we obtain a new construction of the affine minuscule modules from [Cha01].

3.1.2. Module structure for affine adjoint representations. Suppose $\mathfrak{g}$ is not of type $A_{n}^{(1)}$. Suppose $r \in$ $I_{0}$ is adjacent to 0 in the Dynkin diagram of $\mathfrak{g}$. Then we say $V\left(\varpi_{r}\right)$ is a affine adjoint representation since $V\left(\varpi_{r}\right) \simeq V\left(\bar{\Lambda}_{r}\right) \oplus V(0)$ as $U_{q}\left(\mathfrak{g}_{0}\right)$-modules and $V\left(\bar{\Lambda}_{r}\right)$ is the adjoint representation of $U_{q}\left(\mathfrak{g}_{0}\right)$.

We require the statistics

$$
\varepsilon_{i}(b)=\max \left\{k \mid \widetilde{e}_{i}^{k} b \neq 0\right\}, \quad \varphi_{i}(b)=\max \left\{k \mid \widetilde{f}_{i}^{k} b \neq 0\right\} .
$$


Recall from [BFKL06], we can identify

$$
B\left(\varpi_{r}\right)=\left\{x_{\beta} \mid \beta \in \Phi\right\} \sqcup\left\{y_{i} \mid i \in I\right\}
$$

where the crystal structure is given by

$$
\begin{aligned}
x_{\beta} \stackrel{i}{\longrightarrow} x_{\beta-\alpha_{i}} & \left(\beta-\alpha_{i} \in \Phi\right), \\
x_{\alpha_{i}} \stackrel{i}{\longrightarrow} y_{i} \stackrel{i}{\longrightarrow} x_{-\alpha_{i}}, &
\end{aligned}
$$

with $\alpha_{0}=\theta$, the highest root of $\Phi^{+}$. Note that $y_{0}$ was denoted by $\emptyset$ in [BFKL06]. Let $i \sim j$ denote $a_{i j} \neq 0$ and $i \neq j$, i.e., the nodes $i$ and $j$ are adjacent in the Dynkin diagram. We define a $U_{q}(\mathfrak{g})$-module $\mathbb{V}\left(\varpi_{r}\right):=\mathbf{k}\left\{v_{b} \mid b \in B\left(\varpi_{r}\right)\right\}$ by

$$
e_{i} v_{b}=\left\{\begin{array}{ll}
0 & \widetilde{e}_{i} b=\mathbf{0}, \\
{\left[\varphi_{i}(b)+1\right]_{i} v_{\widetilde{e}_{i} b}} & \operatorname{wt}\left(\widetilde{e}_{i} b\right) \neq 0, \\
v_{y_{i}}+\sum_{j \sim i} \frac{\left[-a_{i j}\right]_{i}}{[2]_{j}} v_{y_{j}} & \text { otherwise, }
\end{array} \quad f_{i} v_{b}= \begin{cases}0 & \widetilde{f}_{i} b=\mathbf{0}, \\
{\left[\varepsilon_{i}(b)+1\right]_{i} v_{\widetilde{f}_{i} b}} & \operatorname{wt}\left(\widetilde{f}_{i} b\right) \neq 0, \\
v_{y_{i}}+\sum_{j \sim i} \frac{\left[-a_{i j}\right]_{i}}{[2]_{j}} v_{y_{j}} & \text { otherwise } \\
K_{i} v_{b}=q_{i}^{\left\langle h_{i}, \operatorname{wt}(b)\right\rangle} v_{b} . & \end{cases}\right.
$$

Note that $\varphi_{i}(b)+1=\varphi_{i}\left(\widetilde{e}_{i} b\right)$ and $\varepsilon_{i}(b)+1=\varepsilon_{i}\left(\widetilde{f}_{i} b\right)$.

Proposition 3.4. $\mathbb{V}\left(\varpi_{r}\right)$ is a $U_{q}^{\prime}(\mathfrak{g})$-module.

Proof. For ease of notation, we denote $v_{\beta}:=v_{x_{\beta}}$. We verify the relation

$$
e_{i} f_{j}-f_{i} e_{i}=\delta_{i j} \frac{K_{i}-K_{i}^{-1}}{q_{i}-q_{i}^{-1}}
$$

and leave the rest to the reader as the computation is similar.

We consider $\beta \in \Phi^{+}$and note that the relation holds for $\beta \in \Phi^{-}$by the natural duality $e_{i} \leftrightarrow f_{i}$ and $v_{\alpha} \leftrightarrow v_{-\alpha}$. If $\beta+\alpha_{i}-\alpha_{j} \notin \Phi^{+} \sqcup\{0\}$, then $\left(e_{i} f_{j}-f_{j} e_{i}\right) v_{\beta}=0$. Note that this occurs only if $i \neq j$, and so

$$
\left(e_{i} f_{j}-f_{j} e_{i}\right) v_{\beta}=0=\delta_{i j} \frac{K_{i}-K_{i}^{-1}}{q_{i}-q_{i}^{-1}} v_{\beta}
$$

as desired. Henceforth we assume $\beta+\alpha_{i}-\alpha_{j} \notin \Phi^{+}$(note that it cannot be 0 by height considerations and $\left.\beta \in \Phi^{+}\right)$. If $\beta-\alpha_{j} \notin \Phi^{+} \sqcup\{0\}$, then $\varphi_{i}\left(x_{\beta}\right)=0$ and

$$
\left(e_{i} f_{j}-f_{j} e_{i}\right) v_{\beta}=-\delta_{i j}\left[\varepsilon_{i}\left(x_{\beta}\right)\right]_{i}\left[\varphi_{i}\left(x_{\beta}\right)+1\right]_{i} v_{\beta}=-\delta_{i j}\left[\varepsilon_{i}\left(x_{\beta}\right)\right]_{i} v_{\beta} .
$$

Recall from [Kas90, Kas91] that $\varphi_{i}(b)-\varepsilon_{i}(b)=\left\langle h_{i}, \operatorname{wt}(b)\right\rangle$ for all $b \in B\left(\varpi_{r}\right)$. So we have

$$
\begin{aligned}
\delta_{i j} \frac{K_{i}-K_{i}^{-1}}{q_{i}-q_{i}^{-1}} v_{\beta} & =\delta_{i j} \frac{q_{i}^{\left\langle h_{i}, \mathrm{wt}(b)\right\rangle}-q_{i}^{-\left\langle h_{i}, \mathrm{wt}(b)\right\rangle}}{q_{i}-q_{i}^{-1}} v_{\beta}=\delta_{i j} \frac{q_{i}^{\varphi_{i}\left(x_{\beta}\right)} q_{i}^{-\varepsilon_{i}\left(x_{\beta}\right)}-q_{i}^{\varepsilon_{i}\left(x_{\beta}\right)} q_{i}^{-\varphi_{i}\left(x_{\beta}\right)}}{q_{i}-q_{i}^{-1}} v_{\beta} \\
& =\delta_{i j} \frac{q_{i}^{-\varepsilon_{i}\left(x_{\beta}\right)}-q_{i}^{\varepsilon_{i}\left(x_{\beta}\right)}}{q_{i}-q_{i}^{-1}} v_{\beta}=-\delta_{i j}\left[\varepsilon_{i}\left(x_{\beta}\right)\right]_{i} v_{\beta} .
\end{aligned}
$$


Similar if $\beta+\alpha_{i} \notin \Phi^{+} \sqcup\{0\}$. Now suppose $\beta+\alpha_{i}, \beta-\alpha_{j} \in \Phi^{+} \sqcup\{0\}$. Therefore, we must either have $i=j$ or $a_{i j}=a_{j i}=0$ by examining each irreducible finite root system. Note that if $i \neq j$, then we have

$$
\varphi_{i}\left(\widetilde{f}_{j} x_{\beta}\right)=\varphi_{i}\left(x_{\beta}\right), \quad \varepsilon_{j}\left(\widetilde{e}_{i} x_{\beta}\right)=\varepsilon_{j}\left(x_{\beta}\right),
$$

and by direct computation, we have

$$
\left(e_{i} f_{j}-f_{j} e_{i}\right) v_{\beta}=\left(\left[\varepsilon_{j}\left(x_{\beta}\right)+1\right]_{j}\left[\varphi_{i}\left(x_{\beta}\right)+1\right]_{i}-\left[\varepsilon_{j}\left(x_{\beta}\right)+1\right]_{j}\left[\varphi_{i}\left(x_{\beta}\right)+1\right]_{i}\right) v_{\beta-\alpha_{j}+\alpha_{i}}=0
$$

as desired. Now we consider the case $i=j$, and so we have

$$
\begin{aligned}
\left(e_{i} f_{i}-f_{i} e_{i}\right) v_{\beta} & =\left(\left[\varepsilon_{i}\left(x_{\beta}\right)+1\right]_{i}\left[\varphi_{i}\left(x_{\beta}\right)\right]_{i}-\left[\varepsilon_{i}\left(x_{\beta}\right)\right]_{i}\left[\varphi_{i}\left(x_{\beta}\right)+1\right]_{i}\right) \\
& =\frac{q_{i}^{\varphi_{i}\left(x_{\beta}\right)} q_{i}^{-\varepsilon_{i}\left(x_{\beta}\right)}-q_{i}^{\varepsilon_{i}\left(x_{\beta}\right)} q_{i}^{-\varphi_{i}\left(x_{\beta}\right)}}{q_{i}-q_{i}^{-1}} v_{\beta} \\
& =\frac{q_{i}^{\left\langle h_{i}, \operatorname{wt}(b)\right\rangle}-q_{i}^{-\left\langle h_{i}, \operatorname{wt}(b)\right\rangle}}{q_{i}-q_{i}^{-1}} v_{\beta}=\delta_{i j} \frac{K_{i}-K_{i}^{-1}}{q_{i}-q_{i}^{-1}} v_{\beta} .
\end{aligned}
$$

Note that this also covers the case when $\beta=\alpha_{i}$ as $e_{i} v_{y_{j}}=f_{i} v_{y_{i}}=0$ and $\varphi_{i}\left(x_{\alpha_{i}}\right)=0$.

Now if we consider $v_{y_{i}}$, the computation is similar to the above except for when $i=j$, where we will have the extra contribution of

$$
\sum_{k \sim i} \frac{\left[-a_{i k}\right]_{i}}{[2]_{k}} v_{y_{k}}-\sum_{k \sim i} \frac{\left[-a_{i k}\right]_{i}}{[2]_{k}} v_{y_{k}}=0
$$

Thus the claim follows.

Remark 3.5. We note that we can explicitly verify the specific $\mathbb{V}\left(\varpi_{r}\right)$, in the sense that it is a finite computation to show the relations hold, that we use in this paper are indeed $U_{q}^{\prime}(\mathfrak{g})$-modules and do not require the general result.

Proposition 3.6. We have $\mathbb{V}\left(\varpi_{r}\right) \simeq V\left(\varpi_{r}\right)$.

Proof. The proof is similar to Proposition 3.3 except we have to consider when the result of applying $e_{i}$ or $f_{i}$ to a weight vector results in the 0 weight space. By the weight space decomposition of $\mathbb{V}\left(\varpi_{r}\right)$ and the proof of Proposition 3.3, it is sufficient to consider vectors of the form

$$
v=\sum_{i \in I} \xi_{i} v_{\alpha_{i}} \in \mathbb{V}\left(\varpi_{r}\right)
$$

and show that $f_{i} v \neq 0$ for all $i \in I$ as the result for $e_{i} v$ is similar. However, we have

$$
f_{i} v=\left(\xi_{i}+\sum_{j \sim i} \xi_{j} \frac{\left[-a_{j i}\right]_{j}}{[2]_{i}}\right) v_{y_{i}}+\xi_{i}\left(\sum_{j \sim i} \frac{\left[-a_{i j}\right]_{i}}{[2]_{j}}\right) v_{y_{j}},
$$

which is non-zero if $\xi_{i} \neq 0$. Hence, $v \neq 0$ if and only if there exists an $i \in I$ such that $f_{i} v \neq 0$. Hence $\mathbb{V}\left(\varpi_{r}\right)$ is simple.

From Proposition 3.6, we obtain a new construction of the affine adjoint modules from [Cha01]. 
3.2. $R$-matrices. In this subsection, we recall the notion of $R$-matrices for quantum affine algebras following $[\operatorname{Kas} 02, \S 8]$. For $M, N \in \mathcal{C}_{\mathfrak{g}}$, there is a morphism of $\mathbf{k}\left[\left[z_{N} / z_{M}\right]\right] \otimes_{\mathbf{k}\left[z_{N} / z_{M}\right]} \mathbf{k}\left[z_{M}^{ \pm 1}, z_{N}^{ \pm 1}\right] \otimes U_{q}^{\prime}(\mathfrak{g})$ modules, denoted by $R_{M_{z_{M}}, N_{z_{N}}}^{\mathrm{univ}}$ and called the universal $R$-matrix:

$$
R_{M_{z_{M}}, N_{z_{N}}}^{\mathrm{univ}}: \mathbf{k}\left[\left[z_{N} / z_{M}\right]\right] \otimes_{\mathbf{k}\left[z_{N} / z_{M}\right]}\left(M_{z_{M}} \otimes N_{z_{N}}\right) \rightarrow \mathbf{k}\left[\left[z_{N} / z_{M}\right]\right] \otimes_{\mathbf{k}\left[z_{N} / z_{M}\right]}\left(N_{z_{N}} \otimes M_{z_{M}}\right) .
$$

We say that $R_{M_{z_{M}}, N_{z_{N}}}^{\text {univ }}$ is rationally renormalizable if there exist $a \in \mathbf{k}\left(z_{N} / z_{M}\right)$ and a $\mathbf{k}\left[z_{M}^{ \pm 1}, z_{N}^{ \pm 1}\right] \otimes$ $U_{q}^{\prime}(\mathfrak{g})$-module homomorphism

$$
R_{M_{z_{M}}, N_{z_{N}}}^{\mathrm{ren}}: M_{z_{M}} \otimes N_{z_{N}} \rightarrow N_{z_{N}} \otimes M_{z_{M}}
$$

such that $R_{M_{z_{M}}, N_{z_{N}}}^{\mathrm{ren}}=a R_{M_{z_{M}}, N_{z_{N}}}^{\mathrm{univ}}$. Then we can choose $R_{M_{z_{M}}, N_{z_{N}}}^{\mathrm{ren}}$ so that for any $a_{M}, a_{N} \in \mathbf{k}^{\times}$, the specialization of $R_{M_{z_{M}}, N_{z_{N}}}^{\mathrm{ren}}$ at $z_{M}=a_{M}, z_{N}=a_{N}$,

$$
\left.R_{M_{z_{M}}, N_{z_{N}}}^{\mathrm{ren}}\right|_{z_{M}=a_{M}, z_{N}=a_{N}}: M_{a_{M}} \otimes N_{a_{N}} \rightarrow N_{a_{N}} \otimes M_{a_{M}}
$$

does not vanish provided that $M$ and $N$ are non-zero $U_{q}^{\prime}(\mathfrak{g})$-modules in $\mathcal{C}_{\mathfrak{g}}$. Such an $R^{\text {ren }}$ is unique up to $\mathbf{k}\left[\left(z_{M} / z_{N}\right)^{ \pm 1}\right]^{\times}=\bigsqcup_{n \in \mathbb{Z}} \mathbf{k}^{\times} z_{M}^{n} z_{N}^{-n}$, and it is called a renormalized $R$-matrix. For more details, we refer the reader to [EFK98].

We denote by

$$
\mathbf{r}_{M, N}:=\left.R_{m_{z_{M}}, N_{z_{N}}}^{\mathrm{ren}}\right|_{z_{M}=1, z_{N}=1}: M \otimes N \rightarrow N \otimes M
$$

and call it the R-matrix. By definition $\mathbf{r}_{M, N}$ never vanishes. When $M=V\left(\varpi_{i}\right)$ and $N=V\left(\varpi_{j}\right)$, we simply denote

$$
\mathbf{r}_{i, j}\left(z_{V\left(\varpi_{i}\right)}, z_{V\left(\varpi_{j}\right)}\right):=R_{V\left(\varpi_{i}\right) z_{V\left(\varpi_{i}\right)}}^{\mathrm{ren}}, V\left(\varpi_{j}\right)_{z_{V(\varpi y)}} .
$$

For simple $U_{q}^{\prime}(\mathfrak{g})$-modules $M$ and $N$ in $\mathcal{C}_{\mathfrak{g}}$, the universal $R$-matrix $R_{M_{z_{M}}, N_{z_{N}}}^{\text {univ }}$ is rationally renormalizable. Then, for dominant extremal weight vectors $u_{M}$ and $u_{N}$ of $M$ and $N$, there exists $a_{M, N}\left(z_{N} / z_{M}\right) \in \mathbf{k}\left[\left[z_{N} / z_{M}\right]\right]^{\times}$such that

$$
\left.\left.R_{M_{z_{M}}, N_{z_{N}}}^{\text {univ }}\left(\left(u_{M}\right)_{z_{M}} \otimes\left(u_{N}\right)_{z_{N}}\right)\right)=a_{M, N}\left(z_{N} / z_{M}\right)\left(\left(u_{N}\right)_{z_{N}} \otimes\left(u_{M}\right)_{z_{M}}\right)\right) .
$$

Then the map $R_{M_{z_{M}}, N_{z_{N}}}^{\text {norm }}:=a_{M, N}\left(z_{N} / z_{M}\right)^{-1} R_{M_{z_{M}}, N_{z_{N}}}^{\text {univ }}$ is the unique $\mathbf{k}\left(z_{M}, z_{N}\right) \otimes_{\mathbf{k}\left[z_{M}^{ \pm 1}, z_{N}^{ \pm 1}\right]} U_{q}^{\prime}(\mathfrak{g})$ module homomorphism sending $\left(\left(u_{M}\right)_{z_{M}} \otimes\left(u_{N}\right)_{z_{N}}\right)$ to $\left(\left(u_{N}\right)_{z_{N}} \otimes\left(u_{M}\right)_{z_{M}}\right)$. It is known that $\mathbf{k}\left(z_{M}, z_{N}\right) \otimes_{\mathbf{k}\left[z_{M}^{ \pm 1}, z_{N}^{ \pm 1}\right]}\left(M_{z_{M}} \otimes N_{z_{N}}\right)$ is simple $\mathbf{k}\left(z_{M}, z_{N}\right) \otimes_{\mathbf{k}\left[z_{M}^{ \pm 1}, z_{N}^{ \pm 1}\right]} U_{q}^{\prime}(\mathfrak{g})$-module ([Kas02, Proposition 9.5]). We call $R_{M_{z_{M}}, N_{z_{N}}}^{\text {norm }}$ the normalized $R$-matrix.

Let us denote by $d_{M, N}(u) \in \mathbf{k}[u]$ a monic polynomial of the smallest degree such that the image $d_{M, N}\left(z_{N} / z_{M}\right) R_{M_{z_{M}}, N_{z_{N}}}^{\text {norm }}$ is contained in $N_{z_{N}} \otimes M_{z_{M}}$. We call $d_{M, N}$ the denominator of $R_{M_{z_{M}}, N_{z_{N}}}^{\text {norm }}$. Then,

$$
d_{M, N}\left(z_{N} / z_{M}\right) R_{M_{z_{M}}, N_{z_{N}}}^{\text {norm }}: M_{z_{M}} \otimes N_{z_{N}} \rightarrow N_{z_{N}} \otimes M_{z_{M}}
$$

is a renormalized $R$-matrix, and

$$
\mathbf{r}_{M, N}: M \otimes N \rightarrow N \otimes M
$$

is equal to $\left.d_{M, N}\left(z_{N} / z_{M}\right) R_{M_{z_{M}}, N_{z_{N}}}^{\text {norm }}\right|_{z_{M}=1, z_{N}=1}$ up to a constant multiple. 
Note that the following diagram is commutative

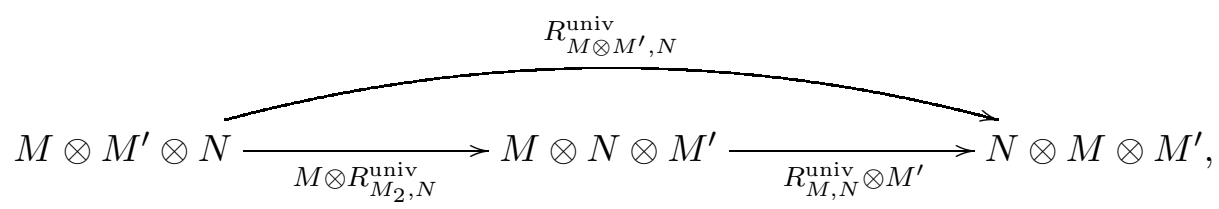

for $M, M^{\prime}, N \in \mathcal{C}_{\mathfrak{g}}$. Hence, if $R_{M_{z_{M}}, N_{z_{N}}}^{\text {univ }}$ and $R_{M^{\prime} z_{M^{\prime}}, N_{z_{N}}}^{\text {univ }}$ are rationally renormalizable, then $R_{\left(M \otimes M^{\prime}\right)_{z}, N_{z_{N}}}^{\text {univ }}$ is also. In particular, if $M, M^{\prime}$ and $N$ are simple modules in $\mathcal{C}_{\mathfrak{g}}$, then $R_{\left(M \otimes M^{\prime}\right)_{z_{1}}, N_{z_{2}}}^{\text {univ }}$ is rationally renormalizable.

Lemma 3.7 ([KO18, Lemma 7.3]). Let $U_{q}^{\prime}(\mathfrak{g})$ be a quantum affine algebra, and let $V$ and $W$ be good $U_{q}^{\prime}(\mathfrak{g})$-modules. If the normalized $R$-matrix $R_{V, W}^{\mathrm{norm}}(z)$ has a simple pole at $z=$ a for some $a \in \mathbf{k}^{\times}$, then we have

$$
\operatorname{Im}\left(\left.(z-a) R_{V, W}^{\text {norm }}\right|_{z=a}\right)=\operatorname{Ker}\left(\left.R_{W, V}^{\text {norm }}\right|_{z=a}\right) .
$$

Moreover, the tensor product $V \otimes W_{a}$ is of composition length 2 .

Lemma 3.8 ([AK97, Lemma C.15]). Let $V^{\prime}, V^{\prime \prime}, V$ and $W$ be simple $U_{q}^{\prime}(\mathfrak{g})$-modules in $\mathcal{C}_{\mathfrak{g}}$. Assume that we have a surjective $U_{q}^{\prime}(\mathfrak{g})$-homomorphism

$$
V^{\prime} \otimes V^{\prime \prime} \rightarrow V
$$

Then we have

$$
\frac{d_{W, V^{\prime}}(z) d_{W, V^{\prime \prime}}(z) a_{W, V}(z)}{d_{W, V}(z) a_{W, V^{\prime}}(z) a_{W, V^{\prime \prime}}(z)} \quad \text { and } \quad \frac{d_{V^{\prime}, W}(z) d_{V^{\prime \prime}, W}(z) a_{V, W}(z)}{d_{V, W}(z) a_{V^{\prime}, W}(z) a_{V^{\prime \prime}, W}(z)} \in \mathbf{k}\left[z^{ \pm 1}\right] .
$$

For $i, j \in I_{0}$, we denote by $d_{i, j}(z)$ by the denominator $d_{V\left(\varpi_{i}\right), V\left(\varpi_{j}\right)}$ between the fundamental representations $V\left(\varpi_{i}\right)$ and $V\left(\varpi_{j}\right)$. We write

$$
d_{i, j}(z)=\prod_{\nu}\left(z-x_{\nu}\right) \quad \text { and } \quad d_{i^{*}, j}(z)=\prod_{\nu}\left(z-y_{\nu}\right) .
$$

For rational functions $f, g \in \mathbf{k}(z)$, we write $f \equiv g$ if there exists an element $a \in \mathbf{k}\left[z^{ \pm 1}\right]^{\times}$such that $f=a g$.

Lemma 3.9 ([AK97]). For $k, l \in I_{0}$, we have

$$
\begin{aligned}
a_{k, l}(z) a_{k^{*}, l}\left(\left(p^{*}\right)^{-1} z\right) & \equiv \frac{d_{k, l}(z)}{d_{k^{*}, l}\left(p^{*} z^{-1}\right)}, \\
a_{k, l}(z) & =q^{\left(\varpi_{k}, \varpi_{l}\right)} \prod_{\nu} \frac{\left(p^{*} y_{\nu} z ; p^{* 2}\right)_{\infty}\left(p^{*} y_{\nu}^{-1} z ; p^{* 2}\right)_{\infty}}{\left(x_{\nu} z ; p^{* 2}\right)_{\infty}\left(p^{* 2} x_{\nu}^{-1} z ; p^{* 2}\right)_{\infty}},
\end{aligned}
$$

where $(z ; x)_{\infty}=\prod_{t=0}^{\infty}\left(1-x^{t} z\right)$ and $a_{k, l}(z):=a_{V\left(\varpi_{k}\right), V\left(\varpi_{l}\right)}$ in $(3.6)$.

3.3. Denominator formulas and folded AR quiver. In this subsection, we shall review the relationship the folded AR quiver with the denominator formulas between fundamental representations. We start this subsection with the following theorem telling that the denominator formulas between fundamental representations play an important roles of representation theory on $\mathcal{C}_{\mathfrak{g}}$.

Theorem 3.10 ([AK97, Cha02, Kas02, VV02] (see also [KKK18, Theorem 2.2.1])).

(1) For good modules $M, N$, the zeros of $d_{M, N}(z)$ belong to $\mathbb{C}\left[\left[q^{1 / m}\right]\right] q^{1 / m}$ for some $m \in \mathbb{Z}_{>0}$. 
(2) Let $M_{k}$ be a good module with a dominant extremal vector $u_{k}$ of weight $\lambda_{k}$, and $a_{k} \in \mathbf{k}^{\times}$for $k=1, \ldots, t$. Assume that $a_{j} / a_{i}$ is not a zero of $d_{M_{i}, M_{j}}(z)$ for any $1 \leq i<j \leq t$. Then the following statements hold.

(i) $\left(M_{1}\right)_{a_{1}} \otimes \cdots \otimes\left(M_{t}\right)_{a_{t}}$ is generated by $u_{1} \otimes \cdots \otimes u_{t}$.

(ii) The head of $\left(M_{1}\right)_{a_{1}} \otimes \cdots \otimes\left(M_{t}\right)_{a_{t}}$ is simple.

(iii) Any non-zero submodule of $\left(M_{t}\right)_{a_{t}} \otimes \cdots \otimes\left(M_{1}\right)_{a_{1}}$ contains the vector $u_{t} \otimes \cdots \otimes u_{1}$.

(iv) The socle of $\left(M_{t}\right)_{a_{t}} \otimes \cdots \otimes\left(M_{1}\right)_{a_{1}}$ is simple.

(v) Let $\mathbf{r}:\left(M_{1}\right)_{a_{1}} \otimes \cdots \otimes\left(M_{t}\right)_{a_{t}} \rightarrow\left(M_{t}\right)_{a_{t}} \otimes \cdots \otimes\left(M_{1}\right)_{a_{1}}$ be the specialization of $R_{M_{1}, \ldots, M_{t}}^{\text {norm }}$ at $z_{k}=a_{k}$. Then the image of $\mathbf{r}$ is simple and it coincides with the head of $\left(M_{1}\right)_{a_{1}} \otimes$ $\cdots \otimes\left(M_{t}\right)_{a_{t}}$ and also with the socle of $\left(M_{t}\right)_{a_{t}} \otimes \cdots \otimes\left(M_{1}\right)_{a_{1}}$.

(3) For a simple integrable $U_{q}^{\prime}(\mathfrak{g})$-module $M$, there exists a unique finite sequence $\left(\left(i_{k}, a_{k}\right) \in\right.$ $\left.I_{0} \times \mathbf{k}^{\times}\right)_{k=1}^{t}$ (up to permutation) such that $d_{i_{k}, i_{k^{\prime}}}\left(a_{k^{\prime}} / a_{k}\right) \neq 0$ for $1 \leq k<k^{\prime} \leq t$ and

$$
M \simeq \operatorname{hd}\left(\bigotimes_{k=1}^{t} V\left(\varpi_{i_{k}}\right)_{a_{k}}\right) .
$$

(4) $d_{k, l}(z)=d_{l, k}(z)=d_{k^{*}, l^{*}}(z)=d_{l^{*}, k^{*}}(z)$ for $k, l \in I_{0}$.

The denominator formulas between fundamental representations over classical quantum affine algebras were calculated in [AK97, DO94, KKK15, Oh15]. Interestingly, such formulas can be read from folded AR quiver, which are explained in the following theorems:

Theorem 3.11 ([Oh15, Oh19, OS16a, OS16b]).

(1) For type $A_{n}^{(1)}, B_{n}^{(1)}, C_{n}^{(1)}$, and $D_{n}^{(1)}$, their denominator formulas between fundamental representations can be read from folded $A R$ quivers $\left(\Gamma_{Q}\right.$ or $\left.\widehat{\Upsilon}_{[\mathscr{Q}]}\right)$ directly in the following sense:

$$
\begin{aligned}
& d_{k, l}^{A_{n}^{(1)}}(z)=D_{k, l}^{\llbracket \Delta \rrbracket}(z ;-q) \times\left(z-p^{*}\right)^{\delta_{k, l^{*}}} \quad \text { for } \llbracket \Delta \rrbracket \text { of type } A_{n}, \\
& d_{k, l}^{D_{n}^{(1)}}(z)=D_{k, l}^{\llbracket \Delta \rrbracket}(z ;-q) \times\left(z-p^{*}\right)^{\delta_{k, l^{*}}} \quad \text { for } \llbracket \Delta \rrbracket \text { of type } D_{n}, \\
& d_{k, l}^{B_{n}^{(1)}}(z)=D_{k, l}^{\llbracket \mathscr{Q} \rrbracket}\left(z ;-q_{s}\right) \times\left(z-p^{*}\right)^{\delta_{k, l}} \quad \text { for } \llbracket \mathscr{Q} \rrbracket \text { of type } A_{2 n-1} \text {, } \\
& d_{k, l}^{C_{n}^{(1)}}(z)=D_{k, l}^{\llbracket \mathscr{Q} \rrbracket}\left(z ;-q_{s}\right) \times\left(z-p^{*}\right)^{\delta_{k, l}} \quad \text { for } \llbracket \mathscr{Q} \rrbracket \text { of type } D_{n+1} \text {. }
\end{aligned}
$$

(2) For type $A_{n}^{(2)}$ and $D_{n+1}^{(2)}$, their denominator formulas between fundamental representations can be obtained from the one of type $A_{n}^{(1)}$ and $D_{n+1}^{(1)}$ respectively. Thus the formulas can be also read from AR quivers:

$$
\begin{aligned}
d_{k, l}^{A_{n}^{(2)}}(z) & =D_{k, l}^{\llbracket \Delta \rrbracket}(z ;-q) D_{n+1-k, l}^{\llbracket \Delta \rrbracket}\left((-1)^{n} z ;-q\right) \times\left(z-p^{*}\right)^{\delta_{k, l}} \\
& =d_{k, l}^{A_{n}^{(1)}}(z) d_{n+1-k, l}^{A_{n}^{(1)}}\left((-1)^{n} z\right) \\
d_{k, l}^{D_{n+1}^{(2)}}(z) & = \begin{cases}D_{n, n+1}^{\llbracket \Delta \rrbracket}(-z ;-q) D_{n, n}^{\llbracket \Delta \rrbracket}(-z ;-q) \times\left(z-p^{*}\right) & \text { if } k=l=n, \\
D_{k, l}^{\llbracket \Delta \rrbracket}\left(z^{2} ;-q^{2}\right)\left(z-p^{*}\right)^{\delta_{k, l}} & \text { otherwise, }\end{cases}
\end{aligned}
$$

up to sign \pm 1 . 
3.4. Dorey's rule, categorification and folded AR quiver. The morphisms in

$$
\operatorname{Hom}_{U_{q}^{\prime}(\mathfrak{g})}\left(V\left(\varpi_{i}\right)_{a} \otimes V\left(\varpi_{j}\right)_{b}, V\left(\varpi_{k}\right)_{c}\right) \quad \text { for } i, j, k \in I_{0} \text { and } a, b, c \in \mathbf{k}^{\times}
$$

are studied by [CP96, HL10, KKK15, KKKO16, Oh15, Oh19, YZ11] and called Dorey's type morphisms. In [Oh16, Oh17, Oh19], the condition of non-vanishing of the above Hom space are interpreted the positions of $\alpha, \beta, \gamma \in \Phi^{+}$in some $\widehat{\Upsilon}_{\left[\widetilde{\mathfrak{w}}_{0}\right]}$, where $\gamma=\alpha+\beta$, and $\mathfrak{g}$ is a quantum affine algebra of non-exceptional type or $E_{6,7,8}^{(1)}$. We start this subsection with the definition of monoidal subcategories of $\mathscr{C}_{\mathfrak{g}}$, which are closely related to the $r$-cluster points and their (folded) AR-quivers introduced in the earlier parts in this paper.

Recall the map $\bar{\sigma}$ in Definition 2.3. For simplicity, we use the following convention:

For a statement $P, \chi(P)$ is 1 if $P$ is true and 0 if $P$ is false.

Definition 3.12 ([HL10, KKKO16]). Fix $[Q] \in \llbracket \Delta \rrbracket$ of finite type $\mathfrak{g}=A_{n}, D_{n}$ or $E_{6,7,8}$, and a positive root $\beta \in \Phi^{+}$with $\widehat{\Omega}_{[Q]}(\beta)=(i, p)$. For each $\mathfrak{g}^{(t)}(t=1,2,3)$, we take the Dynkin diagram automorphism $\sigma$ of $\Delta$ of type $\mathfrak{g}$ whose order is $t$. We set $U_{q}^{\prime}\left(\mathfrak{g}^{(t)}\right)$-module $V_{Q}^{(t)}(\beta)$ defined as follows:

$$
V_{Q}^{(1)}(\beta):=V\left(\varpi_{i}\right)_{(-q)^{p}}, \quad V_{Q}^{(2)}(\beta):=V\left(\varpi_{i^{\star}}\right)_{\left((-q)^{p}\right)^{\star}}, \quad V_{Q}^{(3)}(\beta):=V\left(\varpi_{i^{\dagger}}\right)_{\left((-q)^{p}\right)^{\dagger}},
$$

where

$$
i^{\star}:=\bar{\sigma}(i), \quad\left((-q)^{p}\right)^{\star}:= \begin{cases}\left(\chi(i \leq \sigma(i))+\chi(i>\sigma(i))(-1)^{n}\right)(-q)^{p} & \text { if } \mathfrak{g}^{(2)}=A_{n}^{(2)}, \\ \left(\delta_{i, \sigma(i)}(\sqrt{-1})^{n-i}+\chi(n-1 \leq i \leq n)(-1)^{i}\right)(-q)^{p} & \text { if } \mathfrak{g}^{(2)}=D_{n}^{(2)}, \\ \left(\chi(i<\sigma(i))-\chi(i>\sigma(i))+\delta_{i, \sigma(i)} \sqrt{-1}\right)(-q)^{p} & \text { if } \mathfrak{g}^{(2)}=E_{6}^{(2)}\end{cases}
$$

$$
i^{\dagger}:=\bar{\sigma}(i), \quad\left((-q)^{p}\right)^{\dagger}:=\left(\delta_{i, 1}-\delta_{i, 2}+\delta_{i, 3} \omega+\delta_{i, 4} \omega^{2}\right)(-q)^{p} \quad \text { if } \mathfrak{g}^{(3)}=D_{4}^{(3)} .
$$

Here $\omega$ denotes the primitive third root of unity.

We define the smallest abelian full subcategory $\mathcal{C}_{Q}^{(t)}(t=1,2,3)$ of $\mathcal{C}_{\mathfrak{g}^{(t)}}$ such that

(a) it is stable by taking subquotient, tensor product and extension,

(b) it contains $V_{Q}^{(t)}(\beta)$ for all $\beta \in \Phi^{+}$.

Theorem 3.13 ([CP96, KKKO16, Oh19]). For $\mathfrak{g}^{(1)}=A_{n}^{(1)}, D_{n}^{(1)}, E_{6,7,8}^{(1)}$ or $\mathfrak{g}^{(2)}=A_{n}^{(2)}$, $D_{n}^{(2)}$, let $\left(i_{1}, x_{1}\right),\left(i_{2}, x_{2}\right),\left(i_{3}, x_{3}\right) \in I_{0} \times \mathbf{k}^{\times}$. Then

$$
\operatorname{dim}_{\mathbf{k}}\left(\operatorname{Hom}_{U_{q}^{\prime}\left(\mathfrak{g}^{(t)}\right)}\left(V^{(t)}\left(\varpi_{i_{2}}\right)_{x_{2}} \otimes V^{(t)}\left(\varpi_{i_{1}}\right)_{x_{1}}, V^{(t)}\left(\varpi_{i_{3}}\right)_{x_{3}}\right)\right)=1 \quad(t=1,2)
$$

if and only if there exists a Dynkin quiver $Q$ and $\alpha, \beta, \gamma \in \Phi_{Q}^{+}$such that

(1) $\alpha \prec_{[Q]} \beta$ and $\alpha+\beta=\gamma$,

(2) $V^{(t)}\left(\varpi_{i_{2}}\right)_{x_{2}}=V_{Q}^{(t)}(\beta)_{a}, V^{(t)}\left(\varpi_{i_{1}}\right)_{x_{1}}=V_{Q}^{(t)}(\alpha)_{a}, V^{(t)}\left(\varpi_{i_{3}}\right)_{x_{3}}=V_{Q}^{(t)}(\gamma)_{a}$ for some $a \in \mathbf{k}^{\times}$.

Theorem 3.14 ([HL15, KKKO16]). Let $Q$ be a Dynkin quiver of finite type $A_{n}, D_{n}(t=1,2)$ and $E_{6,7,8}(t=1)$.

(1) $\left[\mathcal{C}_{Q}^{(t)}\right] \simeq U_{\mathbb{A}}(\mathrm{g})^{\vee}$, where $\mathrm{g}$ denotes the simple Lie algebra of the same type and $\left[\mathcal{C}_{Q}^{(t)}\right]$ denotes the Grothendieck ring of $\mathcal{C}_{Q}^{(t)}$. 
(2) The dual $P B W$-basis associated to $[Q]$ and the upper global basis of $U_{\mathbb{A}}^{-}(\mathrm{g})^{\vee}$ are categorified by the modules over $U_{q}^{\prime}\left(\mathfrak{g}^{(t)}\right)$ in the following sense:

(i) The set of all simples in $\mathcal{C}_{Q}^{(t)}$ corresponds to the upper global basis of $\left.U_{\mathbb{A}}^{-}(\mathrm{g})^{\vee}\right|_{q=1}$.

(ii) The set

$$
\left\{V_{Q}^{(t)}\left(\beta_{1}\right)^{\otimes m_{1}} \otimes \cdots \otimes V_{Q}^{(t)}\left(\beta_{\mathrm{N}}\right)^{\otimes m_{\mathrm{N}}} \mid \underline{m} \in \mathbb{Z}_{\geq 0}^{\mathrm{N}}\right\}
$$

corresponds to the dual PBW-basis under the isomorphism in (1).

Definition 3.15 (cf. [OS16a, OS16b]).

(1) For any $[\mathscr{Q}] \in \llbracket \mathscr{Q} \rrbracket$ and any positive root $\beta \in \Phi^{+}$of type $A_{2 n-1}, D_{n+1}$ or $E_{6}$, we set the $U_{q}^{\prime}\left(\mathfrak{g}^{(1)}\right)$-module $\left(\mathfrak{g}^{(1)}=B_{n}^{(1)}, C_{n}^{(1)}\right.$ or $\left.F_{4}^{(1)}\right) V_{\mathscr{Q}}(\beta)$ defined as follows: For $\widehat{\Omega}_{\mathscr{Q}}(\beta)=(i, p / 2)$, we define

$$
V_{\mathscr{Q}}(\beta):= \begin{cases}V\left(\varpi_{i}\right)_{(-1)^{i}\left(q^{1 / 2}\right)^{p}} & \text { if } \mathfrak{g}^{(1)}=B_{n}^{(1)} \text { or } F_{4}^{(1)}, \\ V\left(\varpi_{i}\right)_{\left(-q^{1 / 2}\right)^{p}} & \text { if } \mathfrak{g}^{(1)}=C_{n}^{(1)} .\end{cases}
$$

(2) For any $[\mathfrak{Q}] \in \llbracket \mathfrak{Q} \rrbracket$ and any positive root $\beta \in \Phi^{+}$of type $D_{4}$, we set the $U_{q}^{\prime}\left(G_{2}^{(1)}\right)$-module $\left(\mathfrak{g}^{(1)}=G_{2}^{(1)}\right) V_{\mathfrak{Q}}(\beta)$ defined as follows: For $\widehat{\Omega}_{\mathfrak{Q}}(\beta)=(i, p / 3)$, we define

$$
V_{\mathfrak{Q}}(\beta):=V\left(\varpi_{i}\right)_{\left(-q^{1 / 3}\right)^{p}}
$$

We define the smallest abelian full subcategory $\mathscr{C}_{\mathscr{Q}}^{(1)}$ of $\mathcal{C}_{\mathfrak{g}(1)}$ such that

(a) it is stable by taking subquotient, tensor product and extension,

(b) it contains $V_{\mathscr{Q}}(\beta)$ (resp. $\left.V_{\mathfrak{Q}}(\beta)\right)$ for all $\beta \in \Phi^{+}$.

Theorem 3.16 ([CP96, OS16a, OS16b]). Let $(i, x),(j, y),(k, z) \in I_{0} \times \mathbf{k}^{\times}$. Then

$$
\operatorname{dim}_{\mathbf{k}}\left(\operatorname{Hom}_{U_{q}^{\prime}\left(\mathfrak{g}^{(1)}\right)}\left(V\left(\varpi_{j}\right)_{y} \otimes V\left(\varpi_{i}\right)_{x}, V\left(\varpi_{k}\right)_{z}\right)\right)=1 \quad \text { for } \mathfrak{g}^{(1)}=B_{n}^{(1)} \quad\left(\text { resp. } C_{n}^{(1)}\right)
$$

if and only if there exists a twisted adapted class [Q] of type $A_{2 n-1}\left(\right.$ resp. $\left.D_{n+1}\right)$ and $\alpha, \beta, \gamma \in \Phi_{A_{2 n-1}}^{+}$ (resp. $\left.\Phi_{D_{n+1}}^{+}\right)$such that

(1) $(\alpha, \beta)$ is a $[\mathscr{Q}]$-minimal pair of $\gamma$,

(2) $V\left(\varpi_{j}\right)_{y}=V_{\mathscr{Q}}(\beta)_{a}, V\left(\varpi_{i}\right)_{x}=V_{\mathscr{Q}}(\alpha)_{a}, V\left(\varpi_{k}\right)_{z}=V_{\mathscr{Q}}(\gamma)_{a}$ for some $a \in \mathbf{k}^{\times}$.

Theorem 3.17 ([KO18]). Let [Q] be a twisted adapted class of finite type $A_{2 n-1}$ (resp. $D_{n+1}$ ).

(1) $\left[\mathscr{C}_{\mathscr{Q}}^{(1)}\right] \simeq U_{\mathbb{A}}(\mathrm{g})^{\vee}$, where $\mathrm{g}$ denotes the simple Lie algebra of $A_{2 n-1}\left(\right.$ resp. $\left.D_{n+1}\right)$ and $\left[\mathscr{C}_{\mathscr{Q}}^{(1)}\right]$ denotes the Grothendieck ring of $\mathscr{C}_{\mathscr{Q}}^{(1)}$.

(2) The dual PBW-basis associated to [Q2] and the upper global basis of $U_{\mathbb{A}}^{-}(\mathrm{g})^{\vee}$ are categorified by the modules over $U_{q}^{\prime}\left(\mathfrak{g}^{(1)}\right)$ of affine type $B_{n}^{(1)}$ (resp. $\left.C_{n}^{(1)}\right)$ in the following sense:

(i) The set of all simples in $\mathscr{C}_{Q}^{(1)}$ corresponds to the upper global basis of $\left.U_{\mathbb{A}}^{-}(\mathrm{g})^{\vee}\right|_{q=1}$.

(ii) For each $\underline{m} \in \mathbb{Z}_{\geq 0}^{\mathrm{N}}$, define the $U_{q}^{\prime}\left(\mathfrak{g}^{(1)}\right)$-module $V_{\mathscr{Q}}^{(1)}(\underline{m})$ by

$$
V_{\mathscr{Q}}\left(\beta_{1}\right)^{\otimes m_{1}} \otimes \cdots \otimes V_{\mathscr{Q}}\left(\beta_{\mathrm{N}}\right)^{\otimes m_{\mathrm{N}}} \text {. }
$$

Then the set $\left\{V_{\mathscr{Q}}(\underline{m}) \mid \underline{m} \in \mathbb{Z}_{\geq 0}^{\mathrm{N}}\right\}$ corresponds to the dual PBW-basis under the isomorphism in (1). 
In the rest of this paper, we shall prove

(1) analogues of Theorem 3.11 for all exceptional affine types,

(2) analogues of Theorem 3.13 and Theorem 3.14 for both $E_{6}^{(2)}$ and $D_{4}^{(3)}$,

(2') analogues of Theorem 3.16 and Theorem 3.17 for both $F_{4}^{(1)}$ and $G_{2}^{(1)}$,

by analyzing the structure of their fundamental representations and $R$-matrices between them.

\section{Denominator formulas for Quantum affine Algebras}

In this section, we compute the denominator formula $d_{i, j}(z)$ between fundamental representations $V\left(\varpi_{i}\right)$ and $V\left(\varpi_{j}\right)$ by employing the framework of [AK97, KKK18, Oh15]. We first do this for types $G_{2}^{(1)}, D_{4}^{(3)}, E_{6}^{(2)}$, and $F_{4}^{(1)}$ because one can compute $d_{i, j}(z)$ for certain $i, j \in I_{0}$ by hand. Using such basic formulas and Dorey's type morphisms, we can compute all $d_{i, j}(z)$ 's. This strategy will be applied to all exceptional affine types; that is, for the remained cases, we implemented the fundamental modules and the $R$-matrix computation in SAGEMATH [Dev17, SCc08]. Using this code, we compute some of the basic formulas, which we then are able to compute all $d_{i, j}(z)$ 's using Dorey's rule as in the case of $U_{q}^{\prime}\left(G_{2}^{(1)}\right)$ and $U_{q}^{\prime}\left(D_{4}^{(3)}\right)$. We have also verified a number of the computations using our code.

We note here that, using geometric approach, Fujita independently showed the distribution of simple roots for $d_{i, j}(z)$ for $E_{6,7,8}^{(1)}([$ Fuj18]).

4.1. $U_{q}^{\prime}\left(G_{2}^{(1)}\right)$ and $U_{q}^{\prime}\left(D_{4}^{(3)}\right)$. In this subsection, we shall compute all the denominator formulas and show that the formulas can be read from any folded AR quivers of type $D_{4}$.

4.1.1. $U_{q}^{\prime}\left(G_{2}^{(1)}\right)$. For type $G_{2}^{(1)}$, we have $q_{0}=q_{1}=q$ and $q_{2}=q^{1 / 3}$. Furthermore, we have $q_{s}=q^{1 / 3}$ and $p^{*}=q^{4}$.

Recall that the $U_{q}\left(\mathfrak{g}_{0}\right)$-decompositions of $V\left(\varpi_{1}\right)$ and $V\left(\varpi_{2}\right)$ are given as follows:

$$
V\left(\varpi_{1}\right) \simeq V\left(\bar{\Lambda}_{1}\right) \oplus V(0) \quad \text { and } \quad V\left(\varpi_{2}\right) \simeq V\left(\bar{\Lambda}_{2}\right) .
$$

Let us recall the $U_{q}^{\prime}(\mathfrak{g})$-modules structure of $V\left(\varpi_{2}\right)$ whose crystal graph can be depicted by as follows $[\mathrm{MOW} 12]:^{3}$

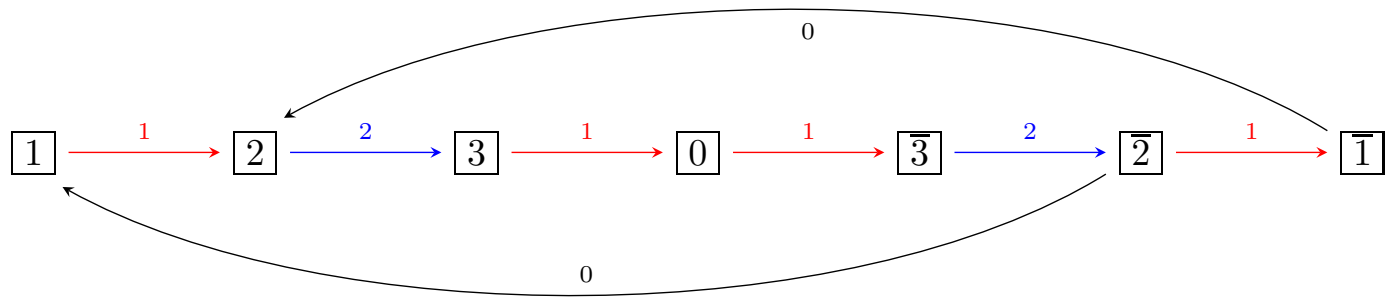

\footnotetext{
${ }^{3}$ This crystal can also be constructed from, e.g., [HN06, Kas02, NS05, NS06b].
} 
where

$$
\begin{aligned}
& e_{0} \overline{1}=\overline{2}, \quad e_{0}, \quad 2=\overline{1}, \quad f_{0} \overline{2}=1, \quad f_{0}[\overline{1}=2,
\end{aligned}
$$

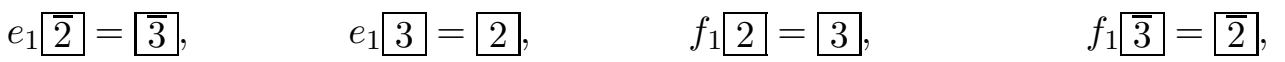

$$
\begin{aligned}
& e_{2} \overline{\overline{1}}=\overline{2}, \quad e_{2} 2=1, \quad e_{2} \overline{\overline{3}}=0, \quad e_{2}, 0=[2]_{2}, 3, \\
& f_{2}\left[\begin{array}{l}
1 \\
2
\end{array}, \quad f_{2}=0, \quad f_{2}=[2]_{2} \overline{\overline{3}}, \quad f_{2}, \quad \overline{2}=\overline{1} .\right.
\end{aligned}
$$

If the action of some basis vector is not written, then we should understand that it vanishes.

The $U_{q}\left(\mathfrak{g}_{0}\right)$-decomposition of $V\left(\varpi_{2}\right) \otimes V\left(\varpi_{2}\right)$ is given as follows (see also [KM94]):

$$
V\left(\varpi_{2}\right) \otimes V\left(\varpi_{2}\right) \simeq V\left(2 \bar{\Lambda}_{2}\right) \oplus V\left(\bar{\Lambda}_{2}\right) \oplus V\left(\bar{\Lambda}_{1}\right) \oplus V(0)
$$

whose $U_{q}\left(G_{2}\right)$-highest weight vectors are given as follows:

$$
\begin{aligned}
& u_{2 \bar{\Lambda}_{2}}:=1 \otimes 1 \text {, } \\
& u_{\bar{\Lambda}_{2}}:=1 \otimes 0-q_{2}^{6}\left[001-q_{2}^{2}[2]_{2} 2 \otimes 3+q_{2}^{5}[2]_{2} 3 \otimes 2,\right. \\
& u_{\bar{\Lambda}_{1}}:=1 \otimes 2+q_{2} 2 \otimes 1 \text {, } \\
& u_{0}:=1 \otimes \overline{1}+q_{2}^{10} \overline{\overline{1}} \otimes 1-q_{2} 2 \otimes \overline{2}-q_{2}^{9} \overline{2} \otimes 2
\end{aligned}
$$

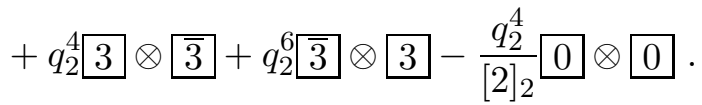

Recall that the $R$-matrix $\mathbf{r}_{2,2}(x, y)$ is the unique $U_{q}^{\prime}(\mathfrak{g})$-module isomorphism

$$
\mathbf{r}_{2,2}(x, y): V\left(\varpi_{2}\right)_{x} \otimes V\left(\varpi_{2}\right)_{y} \rightarrow V\left(\varpi_{2}\right)_{y} \otimes V\left(\varpi_{2}\right)_{x} .
$$

By $U_{q}^{\prime}(\mathfrak{g})$-linearity and $(4.2)$, we have

$$
\begin{aligned}
\mathbf{r}_{2,2}(x, y)\left(u_{2 \bar{\Lambda}_{2}}\right) & =a^{2 \bar{\Lambda}_{2}} u_{2 \bar{\Lambda}_{2}}, & \mathbf{r}_{2,2}(x, y)\left(u_{\bar{\Lambda}_{2}}\right) & =a^{\bar{\Lambda}_{2}} u_{\bar{\Lambda}_{2}}, \\
\mathbf{r}_{2,2}(x, y)\left(u_{\bar{\Lambda}_{1}}\right) & =a^{\bar{\Lambda}_{1}} u_{\bar{\Lambda}_{1}}, & \mathbf{r}_{2,2}(x, y)\left(u_{0}\right) & =a^{0} u_{0} .
\end{aligned}
$$

By direct computations, we have the followings:

Lemma 4.1. In $V\left(\varpi_{2}\right)_{x} \otimes V\left(\varpi_{2}\right)_{y}$, we have the following:

(1) $f_{0} f_{1} f_{2}^{(2)} f_{1}\left(u_{\bar{\Lambda}_{1}}\right)=\left(q_{2}^{-1} y^{-1}-q_{2} x^{-1}\right) u_{2} \bar{\Lambda}_{2}$,

(2) $f_{0} f_{1} f_{2}\left(u_{\bar{\Lambda}_{2}}\right)=[2]_{2} q_{2}^{-2}\left(y^{-1}-q_{2}^{8} x^{-1}\right) u_{2 \bar{\Lambda}_{2}}$,

(3) $f_{0} f_{1} f_{2}^{(2)} f_{1} f_{0}\left(u_{0}\right)=q_{2}^{-4}\left(y^{-1}-q_{2}^{12} x^{-1}\right)\left(y^{-1}-q_{2}^{2} x^{-1}\right) u_{2 \bar{\Lambda}_{2}}$.

Proposition 4.2. Put $z=x^{-1} y$ and denote $\mathbf{r}_{2,2}(z)=\mathbf{r}_{2,2}(x, y)$. Then we have

(1) $a^{2 \bar{\Lambda}_{2}}(z)=\left(z-q_{2}^{2}\right)\left(z-q_{2}^{8}\right)\left(z-q_{2}^{12}\right)$,

(2) $a^{\bar{\Lambda}_{2}}(z)=\left(z-q_{2}^{2}\right)\left(1-q_{2}^{8} z\right)\left(z-q_{2}^{12}\right)$,

(3) $a^{\bar{\Lambda}_{1}}(z)=\left(1-q_{2}^{2} z\right)\left(z-q_{2}^{8}\right)\left(z-q_{2}^{12}\right)$,

(4) $a^{0}(z)=\left(1-q_{2}^{2} z\right)\left(z-q_{2}^{8}\right)\left(1-q_{2}^{12} z\right)$.

Now we can conclude that the denominator $d_{2,2}(z)$ for $U_{q}^{\prime}(\mathfrak{g})$ is given as follows:

$$
d_{2,2}(z)=\left(z-q_{2}^{2}\right)\left(z-q_{2}^{8}\right)\left(z-q_{2}^{12}\right) \text {. }
$$


Remark 4.3. Once we know the module structure of $V\left(\varpi_{i}\right)$ and $V\left(\varpi_{j}\right)$ explicitly, we can compute $d_{i, j}(z)$ (see also $d_{1, n}(z)$ for $D_{n+1}^{(2)}$ in [Oh15, Section 4.2]).

Remark 4.4. By the above proposition, we have

(i) $\operatorname{Im}\left(\mathbf{r}_{2,2}\left(-q_{s}^{-1},-q_{s}^{1}\right)\right) \simeq V\left(\bar{\Lambda}_{1}\right) \oplus V(0)$ as $U_{q}\left(\mathfrak{g}_{0}\right)$-modules,

(ii) $\operatorname{Im}\left(\mathbf{r}_{2,2}\left(\left(-q_{s}\right)^{-4},\left(-q_{s}\right)^{4}\right)\right) \simeq V\left(\bar{\Lambda}_{2}\right)$ as $U_{q}\left(\mathfrak{g}_{0}\right)$-modules.

Proposition 4.5. There exists a injective $U_{q}^{\prime}(\mathfrak{g})$-homomorphism:

$$
V\left(\varpi_{2}\right) \hookrightarrow V\left(\varpi_{2}\right)_{\left(-q_{s}\right)^{4}} \otimes V\left(\varpi_{2}\right)_{\left(-q_{s}\right)^{-4}}
$$

Proof. By [KKKO15a], the socle of $V\left(\varpi_{2}\right)_{a\left(-q_{s}\right)^{4}} \otimes V\left(\varpi_{2}\right)_{a\left(-q_{s}\right)^{-4}}$ is simple. Then (3.1), (4.1) and Remark 4.4 implies that there exists a injective $U_{q}^{\prime}(\mathfrak{g})$-homomorphism

$$
V\left(\varpi_{2}\right) \hookrightarrow V\left(\varpi_{2}\right)_{a\left(-q_{s}\right)^{4}} \otimes V\left(\varpi_{2}\right)_{a\left(-q_{s}\right)^{-4}}
$$

for $a^{2}=1$. Here we use the uniqueness and simplicity of the fundamental representations $V\left(\varpi_{i}\right)$.

If $a=-1$, then $d_{2,2}(z)$ has a root $-q_{s}^{8}$ by taking the dual:

$$
V\left(\varpi_{2}\right) \otimes V\left(\varpi_{1}\right)_{-q_{s}^{8}} \rightarrow V\left(\varpi_{2}\right)_{-q_{s}^{4}} .
$$

However, this can not happen by (4.3).

The following proposition is known as the T-system for type $G_{2}^{(1)}$ proved in [Her06]. However, we shall give a proof since the argument of the proof will be applied to other exceptional types throughout this paper.

Proposition 4.6. There exists a injective $U_{q}^{\prime}(\mathfrak{g})$-homomorphism:

$$
V\left(\varpi_{1}\right) \hookrightarrow V\left(\varpi_{2}\right)_{\left(-q_{s}\right)^{1}} \otimes V\left(\varpi_{2}\right)_{\left(-q_{s}\right)^{-1}}
$$

Proof. By Remark 4.4, there exists a injective $U_{q}^{\prime}(\mathfrak{g})$-homomorphism

$$
V\left(\varpi_{1}\right) \hookrightarrow V\left(\varpi_{2}\right)_{a\left(-q_{s}\right)^{1}} \otimes V\left(\varpi_{2}\right)_{a\left(-q_{s}\right)^{-1}}
$$

for some $a \in \mathbf{k}^{\times}$. As in the above proposition, we have (see [Yam98, Fig. 1])

$$
f_{2}^{(3)} f_{1}\left(u_{\bar{\Lambda}_{1}}\right)=f_{0} e_{1} e_{0}^{(2)}\left(u_{\bar{\Lambda}_{1}}\right)
$$

in $V\left(\varpi_{2}\right)_{a\left(-q_{s}\right)^{1}} \otimes V\left(\varpi_{2}\right)_{a\left(-q_{s}\right)^{-1}}$. On the other hand, by the direct computation, we have

$$
f_{2}^{(3)} f_{1}\left(u_{\bar{\Lambda}_{1}}\right)=2 \otimes\left[\overline{3}-q_{s} \overline{\overline{3}} \otimes 2, \quad f_{0} e_{1} e_{0}^{(2)}\left(u_{\bar{\Lambda}_{1}}\right)=a^{-1}\left(2 \otimes \overline{3}-q_{s} \overline{\overline{3}} \otimes 2\right.\right.
$$

in $V\left(\varpi_{2}\right)_{a\left(-q_{s}\right)^{1}} \otimes V\left(\varpi_{2}\right)_{a\left(-q_{s}\right)^{-1}}$. Thus we can conclude that $a=1$.

Note that $d_{1,1}(z)$ was computed in [Yam98] and we have $d_{2,2}(z)$ by Proposition 4.2:

$$
\begin{aligned}
& d_{1,1}(z)=\left(z-q_{s}^{6}\right)\left(z-q_{s}^{8}\right)\left(z-q_{s}^{10}\right)\left(z-q_{s}^{12}\right), \\
& d_{2,2}(z)=\left(z-q_{s}^{2}\right)\left(z-q_{s}^{8}\right)\left(z-q_{s}^{12}\right) .
\end{aligned}
$$

Now we shall compute $d_{1,2}(z)=d_{2,1}(z)$. By (4.6), we have $a_{1,1}(z):=a_{V\left(\varpi_{1}\right), V\left(\varpi_{1}\right)}$ and $a_{2,2}(z):=$ $a_{V\left(\varpi_{2}\right), V\left(\varpi_{2}\right)}$ in (3.6), we compute the following:

$$
a_{1,1}(z)=\frac{[20][4][22][2][24][0]}{[8][16][10][14][12]^{2}} \quad \text { and } \quad a_{2,2}(z)=\frac{[14][10][20][4][24][0]}{[2][22][8][16][12]^{2}}
$$


where $[a]=\left(\left(-q_{s}\right)^{a} z ; q_{s}^{24}\right)$.

By the direct computation, we have

$$
\left.R_{2,2}^{\text {norm }}(z)(1, \otimes 2]_{z}\right)=\frac{1-q_{s}^{2}}{z-q_{s}^{2}} 1_{z} \otimes 2+\frac{q_{s}(z-1)}{z-q_{s}^{2}} 2 z_{z} \otimes 1 .
$$

Theorem 4.7. We have

$$
a_{1,2}(z)=\frac{[19][5][23][1]}{[7][11][17][13]} \quad \text { and } \quad d_{1,2}(z)=\left(z+q_{s}^{7}\right)\left(z+q_{s}^{11}\right) .
$$

Proof. By (3.7), we have the following commutative diagram:

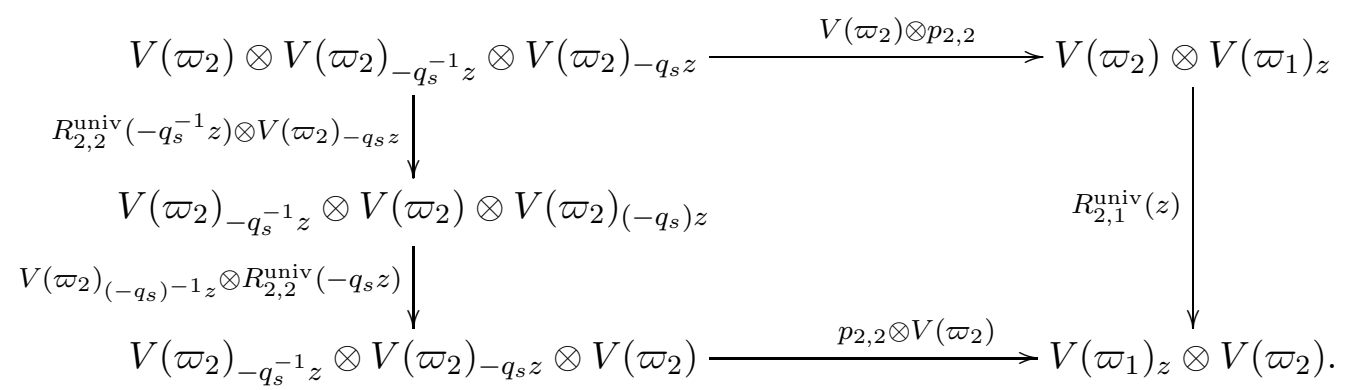

Then we have

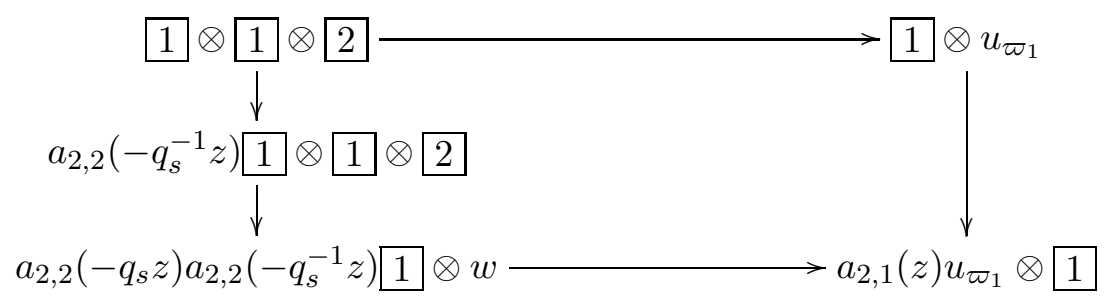

where

$$
w=R_{2,2}^{\text {norm }}\left(-q_{s} z\right)\left(1, \otimes 2 z_{z}\right)=\frac{1-q_{s}^{2}}{-q_{s} z-q_{s}^{2}} 1_{z} \otimes 2+\frac{q_{s}\left(\left(-q_{s}\right) z-1\right)}{-q_{s} z-q_{s}^{2}} 2{ }_{z} \otimes 1 .
$$

Since $1 \otimes 1$ vanishes under the map $p_{2,2}$, we have

$$
a_{2,1}(z)=a_{2,2}\left(-q_{s} z\right) a_{2,2}\left(-q_{s}^{-1} z\right) \frac{q_{s}\left(\left(-q_{s}\right) z-1\right)}{-q_{s} z-q_{s}^{2}} \equiv a_{2,2}\left(-q_{s} z\right) a_{2,2}\left(-q_{s}^{-1} z\right) \frac{[23]}{[-1]} \frac{[1]}{[25]} .
$$

Thus our first assertion follows from (4.7). For the homomorphism (4.5), we have a surjective homomorphism

$$
V\left(\varpi_{2}\right)_{-q_{s}^{-1}} \otimes V\left(\varpi_{2}\right)_{-q_{s}} \rightarrow V\left(\varpi_{1}\right) .
$$

Applying Lemma 3.8 to the above surjective homomorphism, we have

$$
\begin{aligned}
& \mathbf{k}\left[z^{ \pm 1}\right] \ni \frac{d_{2,2}\left(-q_{s}^{-1} z\right) d_{2,2}\left(-q_{s} z\right)}{d_{1,2}(z)} \frac{a_{1,2}(z)}{a_{2,2}\left(-q_{s}^{-1} z\right) a_{2,2}\left(-q_{s} z\right)} \equiv \\
& \frac{\left(z-\left(-q_{s}\right)^{-1}\right)\left(z-\left(-q_{s}\right)^{3}\right)\left(z-\left(-q_{s}\right)^{7}\right)\left(z-\left(-q_{s}\right)^{9}\right)\left(z-\left(-q_{s}\right)^{11}\right)\left(z-\left(-q_{s}\right)^{13}\right)}{d_{1,2}(z)} .
\end{aligned}
$$


By taking the right dual $V\left(\varpi_{2}\right)_{-q_{s}^{11}}$ of $V\left(\varpi_{2}\right)_{-q_{s}^{-1}}$ to (4.5), we have

$$
V\left(\varpi_{1}\right)_{-q_{s}^{-1}} \otimes V\left(\varpi_{2}\right)_{\left(-q_{s}\right)^{10}} \rightarrow V\left(\varpi_{2}\right) .
$$

Similarly, we have

$$
\begin{aligned}
& \frac{d_{2,2}\left(\left(-q_{s}\right)^{10} z\right) d_{1,2}\left(\left(-q_{s}\right)^{-1} z\right)}{d_{2,2}(z)} \frac{a_{2,2}(z)}{a_{1,2}\left(\left(-q_{s}\right)^{-1} z\right) a_{2,2}\left(\left(-q_{s}\right)^{10} z\right)} \\
\equiv & \frac{\left(z-\left(-q_{s}\right)^{18}\right)\left(z-\left(-q_{s}\right)^{22}\right) d_{1,2}\left(\left(-q_{s}\right)^{-1} z\right)}{\left(z-\left(-q_{s}\right)^{6}\right)\left(z-\left(-q_{s}\right)^{10}\right)} \in \mathbf{k}\left[z^{ \pm 1}\right]
\end{aligned}
$$

Thus our second assertion follows from (4.9) and (4.10).

Now we have a type $G_{2}^{(1)}$ analogue for Theorem 3.11.

Theorem 4.8. For any $[\mathfrak{Q}]$ of type $D_{4}$, we have

$$
\begin{aligned}
& d_{1,1}^{G_{2}^{(1)}}(z)=D_{1,1}^{[\mathfrak{Q}]}\left(z ;-q_{s}\right) \times\left(z-q^{4}\right)=\left(z-q_{s}^{6}\right)\left(z-q_{s}^{8}\right)\left(z-q_{s}^{10}\right)\left(z-q_{s}^{12}\right), \\
& d_{1,2}^{G_{2}^{(1)}}(z)=D_{1,2}^{[\mathfrak{Q}]}(z)=\left(z+q_{s}^{7}\right)\left(z+q_{s}^{11}\right), \\
& d_{2,2}^{G_{2}^{(1)}}(z)=D_{2,2}^{[\mathfrak{Q}]}\left(z ;-q_{s}\right) \times\left(z-q^{4}\right)=\left(z-q_{s}^{2}\right)\left(z-q_{s}^{8}\right)\left(z-q_{s}^{12}\right) .
\end{aligned}
$$

4.1.2. $U_{q}^{\prime}\left(D_{4}^{(3)}\right)$. For type $D_{4}^{(3)}$, we have $q_{0}=q_{1}=q, q_{2}=q^{3}$. Furthermore, we have $p^{*}=q^{6}$. Note that the Dynkin diagram for $\mathfrak{g}_{0}$ should be reversed from the one in $(2.1 \mathrm{~d})$.

Recall that the $U_{q}\left(\mathfrak{g}_{0}\right)$-decompositions of $V\left(\varpi_{1}\right)$ and $V\left(\varpi_{2}\right)$ are given as follows:

$$
V\left(\varpi_{1}\right) \simeq V\left(\bar{\Lambda}_{1}\right) \oplus V(0) \quad \text { and } \quad V\left(\varpi_{2}\right) \simeq V\left(\bar{\Lambda}_{2}\right) \oplus V\left(\bar{\Lambda}_{1}\right)^{\oplus 2} \oplus V(0)
$$

Let us recall the $U_{q}^{\prime}\left(D_{4}^{(3)}\right)$-modules structure of $V\left(\varpi_{1}\right)$ whose crystal graph can be depicted as follows:

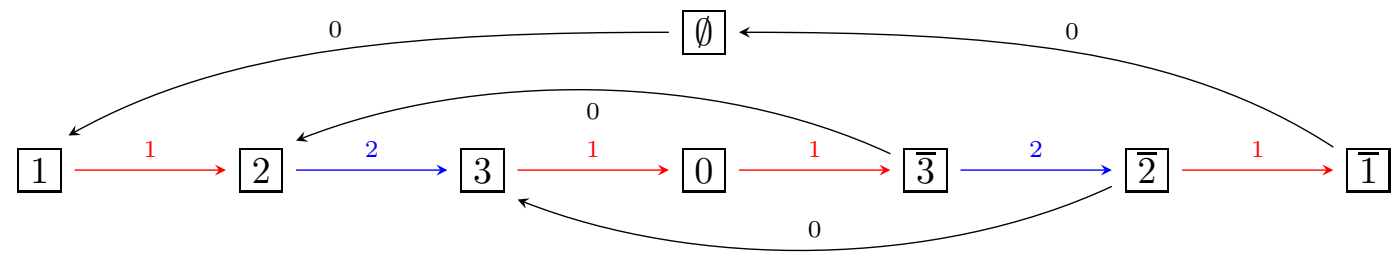

where

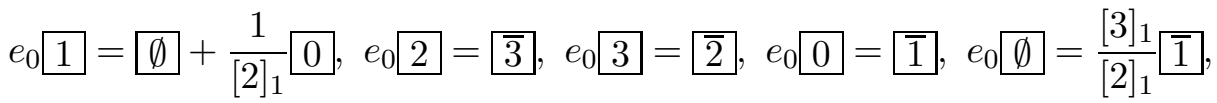

$$
\begin{aligned}
& f_{0} \overline{\overline{1}}=\emptyset+\frac{1}{[2]_{1}} 0, f_{0} \overline{\overline{2}}=3, f_{0} \overline{\overline{3}}=2, f_{0} 0=1, f_{0} \emptyset=\frac{[3]_{1}}{[2]_{1}}[1,
\end{aligned}
$$

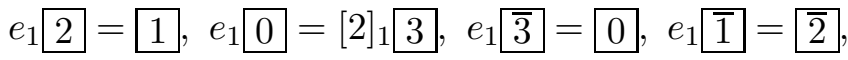

$$
\begin{aligned}
& f_{1} \overline{\overline{2}}=\overline{\overline{1}}, f_{1} 0=[2] \overline{\overline{3}}, f_{1} \overline{3}=0, f_{1}=2 \text {, } \\
& e_{2} \overline{3}=2, e_{2} \overline{\overline{2}}=\overline{\overline{3}}, f_{2} \overline{\overline{3}}=\overline{\overline{2}}, f_{2} 2=3 .
\end{aligned}
$$

In [KMOY07], the existence of following Dorey's type homomorphism is proved:

$$
p_{1,1}: V\left(\varpi_{1}\right)_{-q^{-1}} \otimes V\left(\varpi_{1}\right)_{-q} \rightarrow V\left(\varpi_{2}\right) .
$$


They also computed $d_{1,1}(z)$ also:

$$
d_{1,1}(z)=\left(z-q^{2}\right)\left(z-q^{6}\right)\left(z-\omega q^{4}\right)\left(z-\omega^{2} q^{4}\right)
$$

where $\omega$ is the primitive third root of unity.

By tensoring the left dual $V\left(\varpi_{1}\right)_{-q^{5}}$ of $V\left(\varpi_{1}\right)_{-q^{-1}}$ on $(4.12)$, we have

$$
p_{2,1}^{\prime}: V\left(\varpi_{2}\right) \otimes V\left(\varpi_{1}\right)_{-q^{5}} \rightarrow V\left(\varpi_{1}\right)_{-q} .
$$

Hence we can notice that $-q^{5}$ is a root of $d_{1,2}(z)$.

By (4.13), we have

$$
a_{1,1}(z)=\frac{[8][4][12][0]}{[2][10][6]^{2}} \times \frac{\langle 10\rangle\langle 2\rangle}{\langle 4\rangle\langle 8\rangle}
$$

where

$$
[a]:=\left((-q)^{a} z ; q^{12}\right), \quad\langle a\rangle:=\prod_{s=0}^{\infty}\left(1+(-q)^{12 s+a} z+(-q)^{24 s+2 a} z^{2}\right), \quad\{a\}:=[a]\langle a\rangle .
$$

Lemma 4.9. $a_{1,2}(z)$ and $a_{2,2}(z)$ are given as follows:

$$
a_{1,2}(z)=\frac{\{11\}\{1\}}{\{5\}\{7\}}, \quad a_{2,2}(z)=\frac{\{10\}\{2\}\{12\}\{0\}}{\{4\}\{8\}\{6\}^{2}} .
$$

Proof. Applying the diagrams in the proof of Theorem 4.7, we have

$$
a_{1,1}\left(-q^{-1} z\right) a_{1,1}\left((-q)^{1} z\right) 11 \otimes w \longmapsto a_{1,2}(z)\left(u_{\varpi_{2}}\right)_{z} \otimes 1,
$$

where

$$
R_{1,1}^{\mathrm{norm}}(-q z)\left(11 \otimes 22_{z}\right)=\frac{1-q^{2}}{-q z-q^{2}} L_{z} \otimes 2+\frac{q(1-(-q) z)}{-q z-q^{2}} 2{ }_{z} \otimes 1 .
$$

Since $1 \otimes 1$ vanishes under the map $p_{1,1},(4.16)$ indicates that

$$
\begin{aligned}
a_{1,2}(z) & =a_{1,1}\left(-q^{-1} z\right) a_{1,1}\left((-q)^{1} z\right) \frac{q(-q z-1)}{-q z-q^{2}} \\
& \equiv a_{1,1}\left(-q^{-1} z\right) a_{1,1}\left((-q)^{l-1} z\right) \frac{[1]}{[13]} \frac{[11]}{[-1]} \\
& =\frac{[7][3][11][-1]}{[1][9][5]^{2}} \frac{\langle 9\rangle\langle 1\rangle}{\langle 3\rangle\langle 7\rangle[5][13][1]} \frac{\langle 11\rangle\langle 3\rangle}{[3][11][7]^{2}} \frac{[1]}{\langle 5\rangle\langle 9\rangle} \frac{[11]}{[13]} \\
& =\frac{[1][11]}{[7][5]} \frac{\langle 11\rangle\langle 1\rangle}{\langle 5\rangle\langle 7\rangle}=\frac{\{11\}\{1\}}{\{7\}\{5\}} .
\end{aligned}
$$

Recall that $u_{\varpi_{2}}$ denotes the dominant extremal vector of $V\left(\varpi_{2}\right)$. By direct calculation, one can show that

$$
f_{1}\left(u_{\varpi_{2}} \otimes 1\right)=u_{\varpi_{2}} \otimes 2 \quad \text { and } \quad f_{1}\left(1, \otimes u_{\varpi_{2}}\right)=2 \otimes u_{\varpi_{2}} .
$$

Since $R_{2,1}^{\text {norm }}$ is a $U_{q}^{\prime}(\mathfrak{g})$-homomorphism and sends $u_{\varpi_{2}} \otimes 1$ to $1 \otimes u_{\varpi_{2}}$, we have

$$
R_{2,1}^{\text {norm }}(z)\left(v_{\varpi_{2}} \otimes 2\right)=2 \otimes u_{\varpi_{2}} .
$$


Using the commutative diagram

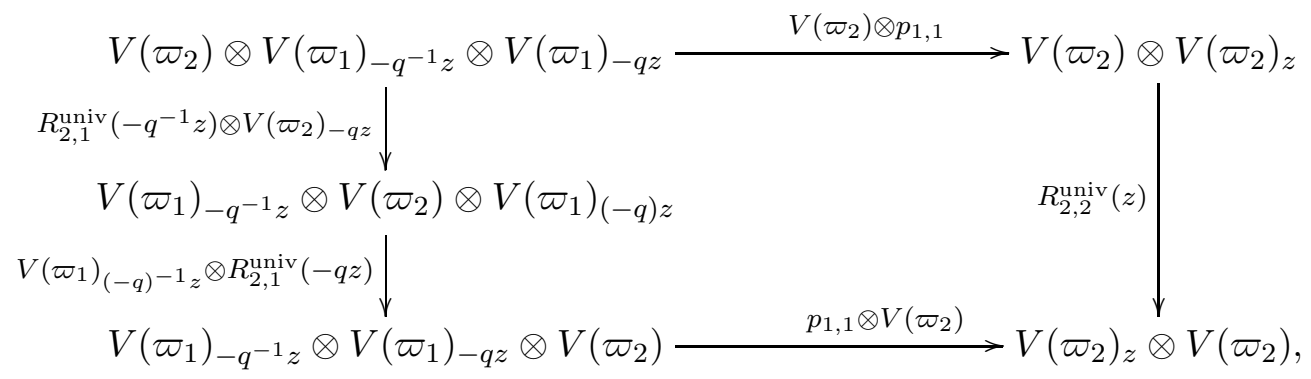

we have

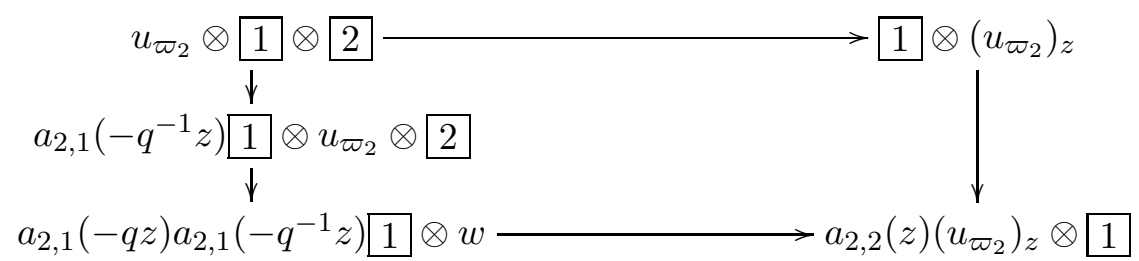

where $w=R_{2,1}^{\text {norm }}\left((-q)^{1} z\right)\left(u_{\varpi_{2}} \otimes 2\right)=2 \otimes u_{\varpi_{2}}$. Thus we have

$$
a_{2,2}(z)=a_{2,1}\left(-q^{-1} z\right) a_{2,1}\left((-q)^{1} z\right) \equiv \frac{\{10\}\{0\}}{\{4\}\{6\}} \frac{\{12\}\{2\}}{\{6\}\{8\}} \equiv \frac{\{10\}\{2\}}{\{4\}\{8\}} \frac{\{12\}\{0\}}{\{6\}^{2}} .
$$

Note that, by $\left[\mathrm{AK} 97\right.$, Eq. (1.7)], we have a $U_{q}^{\prime}(\mathfrak{g})$ isomorphism

$$
V\left(\varpi_{2}\right)_{x} \simeq V\left(\varpi_{2}\right)_{y}
$$

for $x, y \in \mathbf{k}^{\times}$such that $x^{3}=y^{3}$. In [Her10, page 39, arXiv version], it is shown that $q^{2}$ is a root of $d_{2,2}(z)$. Then the following theorem can be proved by applying the same argument of [Oh15, Theorem 5.8].

Theorem 4.10. The denominators of $U_{q}^{\prime}(\mathfrak{g})$ are given as follows:

$$
\begin{aligned}
& d_{1,1}^{D_{4}^{(3)}}(z)=\left(z-q^{2}\right)\left(z-q^{6}\right)\left(z-\omega q^{4}\right)\left(z-\omega^{2} q^{4}\right), \\
& d_{1,2}^{D_{4}^{(3)}}(z)=\left(z^{3}+q^{9}\right)\left(z^{3}+q^{15}\right), \\
& d_{2,2}^{D_{4}^{(3)}}(z)=\left(z^{3}-q^{6}\right)\left(z^{3}-q^{12}\right)^{2}\left(z^{3}-q^{18}\right) .
\end{aligned}
$$

Corollary 4.11. The denominators of $U_{q}^{\prime}(\mathfrak{g})$ can be read from any $\Gamma_{Q}$ of type $D_{4}$ :

$$
\begin{aligned}
& d_{1,1}^{D_{4}^{(3)}}(z)=d_{1,1}^{D_{4}^{(1)}}(z) d_{1,3}^{D_{4}^{(1)}}(\omega z) d_{1,4}^{D_{4}^{(1)}}\left(\omega^{2} z\right)=D_{1,1}^{[Q]}(z ;-q) D_{1,3}^{[Q]}(\omega z ;-q) D_{1,4}^{[Q]}\left(\omega^{2} z ;-q\right) \times\left(z-p^{*}\right), \\
& d_{2,1}^{D_{4}^{(3)}}(z)=d_{2,1}^{D_{4}^{(1)}}(z) d_{2,3}^{D_{4}^{(1)}}(\omega z) d_{2,4}^{D_{4}^{(1)}}\left(\omega^{2} z\right)=D_{2,1}^{[Q]}(z ;-q) D_{2,3}^{[Q]}(\omega z ;-q) D_{2,4}^{[Q]}\left(\omega^{2} z ;-q\right), \\
& d_{2,2}^{D_{4}^{(3)}}(z)=d_{2,2}^{D_{4}^{(1)}}(z) d_{2,2}^{D_{4}^{(1)}}(\omega z) d_{2,2}^{D_{4}^{(1)}}\left(\omega^{2} z\right)=D_{2,2}^{[Q]}(z ;-q) D_{2,2}^{[Q]}(\omega z ;-q) D_{2,2}^{[Q]}\left(\omega^{2} z ;-q\right) \times\left(z-p^{*}\right) .
\end{aligned}
$$


4.2. $U_{q}^{\prime}\left(E_{6}^{(2)}\right)$ and $U_{q}^{\prime}\left(F_{4}^{(1)}\right)$. We first use results obtained from our SAGEMATH implementation by using the $U_{q}^{\prime}(\mathfrak{g})$-module structure of $V\left(\varpi_{i}\right)$ for various indices $i$ given by Proposition 3.6. Then we shall compute the remained denominator formulas almost completely, and show that the formulas can be read from any folded AR quivers of type $E_{6}$.

4.2.1. $U_{q}^{\prime}\left(E_{6}^{(2)}\right)$. For type $E_{6}^{(2)}$, we have $p^{*}=-q^{12}$. Moreover, $\mathfrak{g}_{0}$ is of type $F_{4}$, but the Dynkin diagram should be reversed from the one in (2.1c).

The $U_{q}\left(\mathfrak{g}_{0}\right)$-decompositions of $V\left(\varpi_{1}\right)$ and $V\left(\varpi_{4}\right)$ are given by

$$
V\left(\varpi_{1}\right) \simeq V\left(\bar{\Lambda}_{1}\right) \oplus V(0), \quad V\left(\varpi_{4}\right) \simeq V\left(\bar{\Lambda}_{4}\right) \oplus V\left(\bar{\Lambda}_{1}\right) \oplus V(0) .
$$

For $V\left(\varpi_{1}\right)$, we use the $U_{q}^{\prime}(\mathfrak{g})$-module structure given by Proposition 3.6.

The $U_{q}\left(\mathfrak{g}_{0}\right)$-decomposition of $V\left(\varpi_{1}\right) \otimes V\left(\varpi_{1}\right)$ is given by (see also [KM94])

$$
V\left(\varpi_{1}\right) \otimes V\left(\varpi_{1}\right) \simeq V\left(2 \bar{\Lambda}_{1}\right) \oplus V\left(\bar{\Lambda}_{2}\right) \oplus V\left(\bar{\Lambda}_{4}\right) \oplus V\left(\bar{\Lambda}_{1}\right)^{\oplus 3} \oplus V(0)^{\oplus 2}
$$

whose $U_{q}\left(\mathfrak{g}_{0}\right)$-highest weight vectors can be labeled as follows:

$$
u_{2 \bar{\Lambda}_{1}}, \quad u_{\bar{\Lambda}_{2}}, \quad u_{\bar{\Lambda}_{4}}, \quad u_{\bar{\Lambda}_{1}}^{(t)}(t=1,2,3), \quad u_{0}^{(s)}(s=1,2) .
$$

We can compute the following in $V\left(\varpi_{1}\right)_{x} \otimes V\left(\varpi_{1}\right)_{y}$, where we are considering an extension of scalars to $\mathbf{k}(x, y)$ :

$$
\begin{aligned}
e_{2} e_{3} e_{1} e_{2} e_{*} u_{\bar{\Lambda}_{2}} & =\frac{\left(q^{4}+1\right)^{2}[2]^{10}[3]^{3}\left(y-q^{2} x\right)}{q^{5}(x y)^{3}} u_{2 \bar{\Lambda}_{1}}, \\
e_{4} e_{3}\left(e_{2} e_{1}\right)^{2} e_{3} e_{4} e_{0} e_{*} u_{\bar{\Lambda}_{4}} & =\frac{\left(q^{4}+1\right)^{2}[2]^{11}[3]^{3}\left(q^{6} x+y\right)\left(y-q^{2} x\right)}{q^{6}(x y)^{4}} u_{2 \bar{\Lambda}_{1}}, \\
{\left[\begin{array}{ccc}
e_{0} u_{\bar{\Lambda}_{1}}^{(1)} & e_{0} u_{\bar{\Lambda}_{1}}^{(2)} & e_{0} u_{\bar{\Lambda}_{1}}^{(3)} \\
e_{\times} u_{\bar{\Lambda}_{1}}^{(1)} & e_{\times} u_{\bar{\Lambda}_{1}}^{(2)} & e_{\times} u_{\bar{\Lambda}_{1}}^{(3)} \\
e_{\bullet} u_{\bar{\Lambda}_{1}}^{(1)} & e_{\bullet} u_{\bar{\Lambda}_{1}}^{(2)} & e_{\bullet} u_{\bar{\Lambda}_{1}}^{(3)}
\end{array}\right] } & =\left[\begin{array}{ccc}
\frac{q^{-2}[2] y}{x y} & \frac{[2] x}{x y} & 0 \\
\frac{[2]^{3}[3] x}{(x y)^{2}} & \frac{[2]^{3}[3] y}{q^{2}(x y)^{2}} & \frac{\eta[2]^{5}[3]\left(q^{2} x-y\right)}{(x y)^{2}} \\
\frac{\zeta\left(q^{2} x+2 x+y\right)}{x^{4} y^{3}} & \frac{\zeta\left(q^{2} x+2 q^{2} y+y\right)}{x^{3} y^{4}} & \frac{\zeta[2]\left(q^{1} 2 x-y\right)\left(q^{2} x-y\right)}{(x y)^{4}}
\end{array}\right] u_{2} \bar{\Lambda}_{1}, \\
{\left[\begin{array}{cc}
e_{0}^{2} u_{0}^{(1)} & e_{0}^{2} u_{0}^{(2)} \\
e_{0} e_{\times} u_{0}^{(1)} & e_{0} e_{\times} u_{0}^{(2)}
\end{array}\right] } & =\left[\begin{array}{cc}
\frac{q^{19}[2]\left(q^{3} x^{2}+[2] y^{2}\right)}{(x y)^{2}} & \frac{[2]^{3}}{q x y} \\
\xi & \frac{[2]^{4}[3]\left(q^{2} x^{2}+y^{2}\right)}{q^{2}(x y)^{3}}
\end{array}\right] u_{2} \bar{\Lambda}_{1},
\end{aligned}
$$

where

$$
\begin{aligned}
e_{*} & =e_{2} e_{4} e_{3} e_{1} e_{3} e_{4} e_{2}^{2} e_{1} e_{3} e_{2}^{2} e_{1} e_{0} e_{3} e_{2}^{2} e_{3} e_{1}^{2} e_{4}^{2} e_{3} e_{2}^{2} e_{3} e_{2} e_{3} e_{2} e_{4} e_{1}^{2} e_{0} e_{2} e_{3} e_{2} e_{1} e_{0}^{3}, \\
e_{\bullet} & =e_{1} e_{0} e_{2} e_{3} e_{1} e_{2} e_{*}, \quad e_{\times}=e_{1} e_{2} e_{3} e_{4} e_{2} e_{3} e_{1} e_{2}^{2} e_{1} e_{3} e_{4} e_{2} e_{3} e_{2} e_{1} e_{0}^{3}, \\
\zeta & =q^{-6}[2]^{10}[3]^{3}\left(q^{4}+1\right)^{2}, \quad \eta=q^{6}-2 q^{4}+2 q^{2}-1, \\
\xi & =\frac{[2]^{4}[3]\left(q^{14} \eta x^{2}+\left([3][4]\left(q^{15}+q^{5}\right)-\left(4 q^{18}+6 q^{14}+3 q^{10}+6 q^{6}+4 q^{2}\right)\right) x y-\eta y^{2}\right)}{q(x y)^{3}} .
\end{aligned}
$$


By $U_{q}^{\prime}(\mathfrak{g})$-linearity and (4.19), we have

$$
\begin{aligned}
& \mathbf{r}_{1,1}(x, y)\left(u_{2 \bar{\Lambda}_{1}}\right)=a^{2 \bar{\Lambda}_{1}} u_{2 \bar{\Lambda}_{1}}, \quad \mathbf{r}_{1,1}(x, y)\left(u_{\bar{\Lambda}_{2}}\right)=a^{\bar{\Lambda}_{2}} u_{\bar{\Lambda}_{2}}, \quad \mathbf{r}_{1,1}(x, y)\left(u_{\bar{\Lambda}_{4}}\right)=a^{\bar{\Lambda}_{4}} u_{\bar{\Lambda}_{4}}, \\
& \mathbf{r}_{1,1}(x, y)\left(u_{\bar{\Lambda}_{1}}^{(i)}\right)=\sum_{j=1}^{3} a_{i j}^{\bar{\Lambda}_{1}} u_{\bar{\Lambda}_{1}}^{(j)}(i=1,2,3), \quad \mathbf{r}_{1,1}(x, y)\left(u_{0}^{(i)}\right)=\sum_{j=1}^{2} a_{i j}^{0} u_{0}^{(j)}(i=1,2) .
\end{aligned}
$$

By using (4.20) and that $\mathbf{r}_{1,1}(x, y)$ is a $U_{q}^{\prime}(\mathfrak{g})$-isomorphism, we obtain the following.

Proposition 4.12. Put $z=x^{-1} y$ and denote $\mathbf{r}_{1,1}(z)=\mathbf{r}_{1,1}(x, y)$. Then we have

$$
\begin{aligned}
& a^{2 \bar{\Lambda}_{1}}(z)=\left(z+q^{12}\right)\left(z-q^{8}\right)\left(z+q^{6}\right)\left(z-q^{2}\right) \text {, } \\
& a^{\bar{\Lambda}_{2}}(z)=\left(z+q^{12}\right)\left(z-q^{8}\right)\left(z+q^{6}\right)\left(q^{2} z-1\right), \\
& a^{\bar{\Lambda}_{4}}(z)=\left(z+q^{12}\right)\left(z-q^{8}\right)\left(q^{6} z+1\right)\left(q^{2} z-1\right), \\
& {\left[\begin{array}{c}
a_{11}^{\bar{\Lambda}_{1}}(z) \\
a_{\frac{1}{\Lambda_{1}}}(z) \\
a_{31}^{\Lambda_{1}}(z)
\end{array}\right]=\left[\begin{array}{c}
-\left(q^{12}-q^{10}+q^{8}-q^{4}+q^{2}-z\right)\left(q^{12}+z\right)\left(q^{4}-1\right) z \\
q^{2}\left(-z^{2}+\left(q^{8}-q^{6}+q^{2}-1\right) z+q^{12}\right)\left(q^{12}+z\right)(z-1) \\
q^{3}\left(q^{4}-1\right)\left(q^{6}+1\right)\left(q^{12}+1\right)\left(q^{12}+z\right)\left(q^{2} z-1\right)(z-1)
\end{array}\right],} \\
& {\left[\begin{array}{c}
a_{12}^{\bar{\Lambda}_{1}}(z) \\
a_{\frac{1}{\Lambda_{1}}}(z) \\
a_{32}^{\Lambda_{1}}(z)
\end{array}\right]=\left[\begin{array}{c}
q^{2}\left(-z^{2}+\left(q^{12}-q^{10}+q^{6}-q^{4}\right) z+q^{12}\right)\left(q^{12}+z\right)(z-1) \\
-\left(q^{12}-q^{10} z+q^{8} z-q^{4} z+q^{2} z-z\right)\left(q^{12}+z\right)\left(q^{4}-1\right) z \\
-q\left(q^{4}-1\right)\left(q^{6}+1\right)\left(q^{12}+1\right)\left(q^{12}+z\right)\left(q^{2} z-1\right)(z-1) z
\end{array}\right],} \\
& {\left[\begin{array}{c}
a_{\frac{13}{\Lambda_{1}}}(z) \\
a_{\frac{23}{\Lambda_{1}}}(z) \\
a_{33}^{\Lambda_{1}}(z)
\end{array}\right]=\left[\begin{array}{c}
q\left(q^{2}-1\right)\left(q^{12}+z\right)(z-1) z \\
q\left(q^{2}-1\right)\left(q^{12}+z\right)(z-1) z \\
-\left(q^{12} z^{2}+\left(q^{2}-1\right)\left(q^{12}+q^{6}+1\right) z-q^{2}\right)\left(q^{12}+z\right)\left(q^{2} z-1\right)
\end{array}\right],} \\
& a_{11}^{0}(z)=-q^{26} z^{4}+q^{16}\left(q^{4}-1\right)\left(q^{6}+1\right) z^{3}+\left(-q^{28}+q^{26}+q^{20}-q^{18}+2 q^{14}-q^{10}+q^{8}+q^{2}-1\right) z^{2} \\
& -q^{2}\left(q^{4}-1\right)\left(q^{6}+1\right) z-q^{2} \\
& a_{21}^{0}(z)=\left(q^{2}+1\right)^{2}\left(q^{2}-1\right) q(z+1)(z-1) z, \\
& a_{12}^{0}(z)=\left(q^{2}-1\right) q(z+1)(z-1) \\
& \times\left(-q^{14}\left(q^{4}-q^{2}+1\right)\left(q^{12}+1\right)\left(z^{2}+1\right)\right. \\
& \left.+\left(q^{20}-2 q^{18}+3 q^{16}-3 q^{14}+2 q^{12}-q^{10}+2 q^{8}-3 q^{6}+3 q^{4}-2 q^{2}+1\right) q^{12}[13] z\right), \\
& a_{22}^{0}=\left.a_{11}^{0}\right|_{z \rightarrow z^{-1}} \cdot z^{4} .
\end{aligned}
$$

Thus we have a denominator formulas

$$
d_{1,1}(z)=\left(z+q^{12}\right)\left(z-q^{8}\right)\left(z+q^{6}\right)\left(z-q^{2}\right)
$$

Remark 4.13. By substituting $z$ with a root of $d_{1,1}(z)$ into $a(z)$ 's in Proposition 4.12 , we can observe the following isomorphisms $U_{q}\left(F_{4}\right)$-modules:

$$
\begin{aligned}
\operatorname{Im}\left(\mathbf{r}_{1,1}\left((-q)^{-3}, q^{3}\right)\right) & \simeq V\left(\bar{\Lambda}_{4}\right) \oplus V\left(\bar{\Lambda}_{1}\right) \oplus V(0), \\
\operatorname{Im}\left(\mathbf{r}_{1,1}\left((-q)^{-4},(-q)^{4}\right)\right) & \simeq V\left(\bar{\Lambda}_{1}\right) \oplus V(0) .
\end{aligned}
$$


Proposition 4.14. We have

$$
\begin{aligned}
V\left(\varpi_{1}\right)_{-q^{-1}} \otimes V\left(\varpi_{1}\right)_{-q^{1}} & \rightarrow V\left(\varpi_{2}\right), \\
V\left(\varpi_{4}\right)_{-q^{-1}} \otimes V\left(\varpi_{4}\right)_{-q^{1}} & \rightarrow V\left(\varpi_{3}\right) \simeq V\left(\varpi_{3}\right)_{-1}, \\
V\left(\varpi_{1}\right)_{-q^{-3}} \otimes V\left(\varpi_{1}\right)_{q^{3}} & \rightarrow V\left(\varpi_{4}\right)_{\sqrt{-1}} \simeq V\left(\varpi_{4}\right)_{-\sqrt{-1}}, \\
V\left(\varpi_{1}\right)_{(-q)^{-4}} \otimes V\left(\varpi_{1}\right)_{(-q)^{4}} & \rightarrow V\left(\varpi_{1}\right)_{-1} .
\end{aligned}
$$

Proof. In [Her10, page 39, arXiv version], the Dorey's type morphisms (4.22a) and (4.22b) for $U_{q}^{\prime}\left(E_{6}^{(2)}\right)$ are given. Also, (4.22a) and (4.22b) are known as the twisted $T$-system of type $E_{6}^{(2)}$.

As in Proposition 4.5, Remark 4.13 implies that (4.22c) has to be a Dorey's type homomorphism of the form

$$
V\left(\varpi_{1}\right)_{(-q)^{-3}} \otimes V\left(\varpi_{1}\right)_{q^{3}} \rightarrow V\left(\varpi_{4}\right)_{a}
$$

for $a^{2}=-1$. Thus our assertion follows from the fact that

$$
V\left(\varpi_{4}\right)_{x} \simeq V\left(\varpi_{4}\right)_{y} \quad \text { for } x, y \in \mathbf{k}^{\times} \text {such that } x^{2}=y^{2} .
$$

Similarly, (3.1) implies that (4.22d) has to be a Dorey's type homomorphism of the form

$$
V\left(\varpi_{1}\right)_{(-q)^{-4}} \otimes V\left(\varpi_{1}\right)_{(-q)^{4}} \rightarrow V\left(\varpi_{1}\right)_{a}
$$

for $a^{2}=1$. If $a=1$, then $d_{1,1}(z)$ has a root $-q^{8}$, by taking dual:

$$
V\left(\varpi_{1}\right)_{(-q)^{4}} \hookrightarrow V\left(\varpi_{1}\right)_{1} \otimes V\left(\varpi_{1}\right)_{-q^{-8}} .
$$

However, this can not happen by (4.21).

When we compute the remained denominator formulas, we need to know the image of some vectors under the $R_{i, j}^{\text {norm }}$. Here we collect some computations:

$$
\begin{aligned}
& R_{1,1}^{\text {norm }}\left(u_{\varpi_{1}} \otimes f_{1} u_{\varpi_{1}}\right)=\frac{\left(1-q^{2}\right)}{\left(z-q^{2}\right)}\left(u_{\varpi_{1}} \otimes f_{1} u_{\varpi_{1}}\right)+\frac{q(z-1)}{\left(z-q^{2}\right)}\left(f_{1} u_{\varpi_{1}} \otimes u_{\varpi_{1}}\right), \\
& R_{1,1}^{\text {norm }}\left(u_{\varpi_{1}} \otimes f_{*} u_{\varpi_{1}}\right)=\frac{\left(q^{4}+z\right) q^{2}(z-1)}{\left(q^{6}+z\right)\left(z-q^{2}\right)} f_{*} u_{\varpi_{1}} \otimes u_{\varpi_{1}}+\text { other terms, } \\
& R_{4,4}^{\text {norm }}\left(u_{\varpi_{4}} \otimes f_{4} u_{\varpi_{4}}\right)=\frac{\left(1-q^{4}\right)}{\left(z^{2}-q^{4}\right)}\left(u_{\varpi_{4}} \otimes f_{4} u_{\varpi_{4}}\right)+\frac{q^{2}\left(z^{2}-1\right)}{\left(z^{2}-q^{4}\right)}\left(f_{4} u_{\varpi_{4}} \otimes u_{\varpi_{4}}\right) .
\end{aligned}
$$

Here $f_{*}$ denotes $f_{1} f_{2} f_{3} f_{2} f_{1}$.

By Lemma 3.9 and (4.21), we have

$$
a_{1,1}(z) \equiv \frac{\langle 10\rangle\langle 14\rangle[6][18]\langle 4\rangle\langle 20\rangle[24][0]}{[2][22]\langle 6\rangle\langle 18\rangle[8][16]\langle 12\rangle^{2}}
$$

where

$$
[a]:=\left((-q)^{a} z ; q^{24}\right)_{\infty}, \quad\langle a\rangle:=\left(-(-q)^{a} z ; q^{24}\right)_{\infty}, \quad\{a\}:=[a]\langle a\rangle .
$$


Also, using (4.23a) and $a_{1,1}(z)$, we can obtain

$$
\begin{aligned}
a_{1,2}(z) & \equiv \frac{\langle 9\rangle\langle 15\rangle[5][19]\langle 3\rangle\langle 21\rangle[1][23]}{[3][21]\langle 7\rangle\langle 17\rangle[9][15]\langle 11\rangle\langle 13\rangle}, \\
a_{2,2}(z) & =\frac{[6][18]\langle 4\rangle\langle 20\rangle\langle 2\rangle\langle 22\rangle[24][0]}{\langle 6\rangle\langle 18\rangle[8][16][10][14]\langle 12\rangle^{2}}, \\
d_{1,2}(z) & =\left(z+q^{3}\right)\left(z-q^{5}\right)\left(z-q^{7}\right)\left(z+q^{7}\right)\left(z+q^{9}\right)\left(z-q^{11}\right),
\end{aligned}
$$

by applying the same arguments in Section 4.1.

Proposition 4.15. We have

$$
d_{2,2}(z)=\left(z-q^{2}\right)\left(z-q^{4}\right)\left(z-q^{6}\right)\left(z-q^{8}\right)^{2}\left(z-q^{10}\right)\left(z+q^{4}\right)^{\epsilon}\left(z+q^{6}\right)^{2}\left(z+q^{8}\right)^{\epsilon}\left(z+q^{10}\right)\left(z+q^{12}\right)
$$

for some $\epsilon \in\{0,1\}$.

Proof. Applying Lemma 3.8 to (4.22a), we have

$$
\frac{d_{1,2}\left(-q^{-1} z\right) d_{1,2}(-q z)}{d_{2,2}(z)} \frac{a_{2,2}(z)}{a_{1,2}\left(-q^{-1} z\right) a_{1,2}(-q z)} \in \mathbf{k}\left[z^{ \pm 1}\right]
$$

and hence

$$
\frac{\left(z-q^{2}\right)\left(z-q^{4}\right)\left(z-q^{6}\right)\left(z-q^{8}\right)^{2}\left(z-q^{10}\right)\left(z+q^{4}\right)\left(z+q^{6}\right)^{2}\left(z+q^{8}\right)\left(z+q^{10}\right)\left(z+q^{12}\right)}{d_{2,2}(z)}
$$

is in $\mathbf{k}\left[z^{ \pm 1}\right]$.

Let us consider the surjective homomorphism

$$
V^{(2)}\left(\varpi_{1}\right)_{-q^{-10}} \otimes V^{(2)}\left(\varpi_{2}\right)_{-q} \rightarrow V^{(2)}\left(\varpi_{1}\right),
$$

which can be obtained from (4.22a).

Applying Lemma 3.8 to (4.24), we have

$$
\frac{d_{1,2}\left(-q^{-10} z\right) d_{2,2}(-q z)}{d_{1,2}(z)} \frac{a_{1,2}(z)}{a_{1,2}\left(-q^{-10} z\right) a_{2,2}(-q z)} \in \mathbf{k}\left[z^{ \pm 1}\right] .
$$

Note that

$$
\frac{a_{1,2}(z)}{a_{1,2}\left(-q^{-10} z\right) a_{2,2}(-q z)} \equiv \frac{\left(z-q^{7}\right)\left(z+q^{3}\right)\left(z-q^{1}\right)\left(z+q^{-1}\right)}{\left(z-q^{5}\right)\left(z+q^{7}\right)\left(z-q^{9}\right)\left(z+q^{1}\right)} .
$$

Hence (4.25) can be expressed as follows:

$$
\frac{\left(z+q^{12}\right)\left(z+q^{18}\right)\left(z-q^{16}\right)\left(z-q^{26}\right)\left(z-q^{1}\right)\left(z+q^{-1}\right) d_{2,2}(-q z)}{\left(z-q^{5}\right)^{2}\left(z+q^{7}\right)^{2}\left(z-q^{9}\right)\left(z+q^{1}\right)\left(z+q^{9}\right)\left(z-q^{11}\right)} \in \mathbf{k}\left[z^{ \pm 1}\right] .
$$

Thus, we have $\left(z+q^{6}\right)^{2}\left(z-q^{8}\right)^{2}\left(z+q^{10}\right)\left(z-q^{2}\right)\left(z-q^{10}\right)\left(z+q^{12}\right) \mid d_{2,2}(z)$. Since $\left(z-q^{2}\right) \mid d_{2,2}(z)$ by [Her10, page 39; arxiv version], (3.8) implies our assertion.

We will refine the denominator formula $d_{2,2}(z)$ by using generalized Schur-Weyl duality. More precisely, we will prove that $\epsilon=1$.

Now we shall compute $a_{1,4}(\sqrt{-1} z)$, which needs to be treated more carefully:

Lemma 4.16. We have

$$
a_{1,4}(\sqrt{-1} z)=\frac{\{7\}\{17\}\{3\}\{21\}}{\{5\}\{19\}\{9\}\{15\}}
$$


Proof. Note that the $U_{q}\left(\mathfrak{g}_{0}\right)$ highest weight vector $u_{\Lambda_{4}}$ in $V\left(\varpi_{1}\right) \otimes V\left(\varpi_{1}\right)$ contains a summand $u_{\varpi_{1}} \otimes f_{1} f_{2} f_{3} f_{2} f_{1} u_{\varpi_{1}}$. Now we set $f_{*}=f_{1} f_{2} f_{3} f_{2} f_{1}$ for brevity. By (3.7), we have the following commutative diagrams:

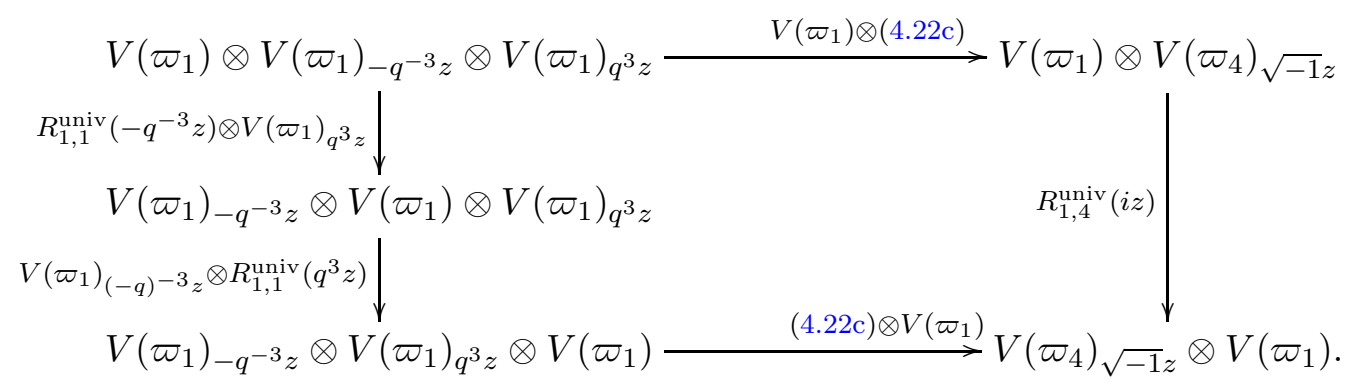

sending

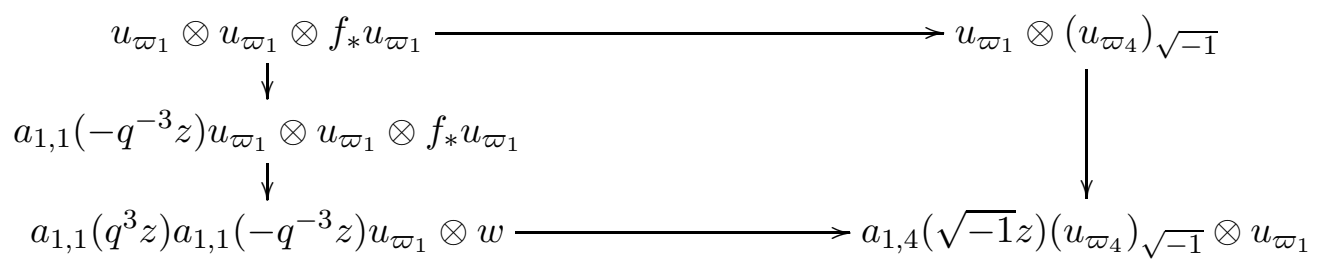

where $w$ is the image of $u_{\varpi_{1}} \otimes f_{*} u_{\varpi_{1}}$ under $R_{1,1}^{\text {norm }}\left(q^{3} z\right)$. Since the remained terms in (4.23b) vanish under $(4.22 \mathrm{c})$, we have

$$
a_{1,4}(\sqrt{-1} z) \equiv a_{1,1}\left(q^{3} z\right) a_{1,1}\left(-q^{-3} z\right) \frac{[21][-1]\langle 3\rangle\langle 25\rangle}{[-3][23]\langle 27\rangle\langle 1\rangle}=\frac{[7][17]\langle 7\rangle\langle 17\rangle[3][21]\langle 3\rangle\langle 21\rangle}{[5][19]\langle 5\rangle\langle 19\rangle[9][15]\langle 9\rangle\langle 15\rangle}
$$

since

$$
\frac{\left(q^{4}+z\right) q^{2}(z-1)}{\left(q^{6}+z\right)\left(z-q^{2}\right)} \equiv \frac{[21][-1]\langle 3\rangle\langle 25\rangle}{[-3][23]\langle 27\rangle\langle 1\rangle}
$$

Hence, the claim follows.

Now using (4.23) and the $a_{i, j}(z)$ values we have computed, we can compute the remaining values of $a_{i, j}(z)$ :

$$
\begin{aligned}
a_{4,4}(z) & =\frac{\{10\}\{14\}\{4\}\{20\}\{24\}\{0\}}{\{2\}\{22\}\{8\}\{16\}\{12\}^{2}}, & a_{2,4}(\sqrt{-1} z) & =\frac{\{2\}\{22\}}{\{10\}\{14\}}, \\
a_{3,4}(z) & \equiv \frac{\{5\}\{19\}\{1\}\{23\}}{\{7\}\{17\}\{11\}\{13\}}, & a_{3,3}(z) & =\frac{\{4\}\{20\}\{2\}\{22\}\{0\}\{24\}}{\{8\}\{16\}\{10\}\{14\}\{12\}^{2}}, \\
a_{2,3}(\sqrt{-1} z) & \equiv \frac{\{3\}\{21\}\{1\}\{23\}}{\{9\}\{15\}\{11\}\{13\}}, & a_{1,3}(\sqrt{-1} z) & =\frac{\{2\}\{22\}}{\{10\}\{14\}} .
\end{aligned}
$$


Also, we can compute all $d_{i, j}(z)$ with some ambiguities (as in Proposition 4.15) will be refined later (except $\epsilon^{\prime}$ in $(4.27 \mathrm{~d})$ ):

$$
\begin{aligned}
& d_{1,4}(z)=\left(z^{2}+q^{10}\right)\left(z^{2}+q^{18}\right), d_{4,4}(z)=\left(z^{2}-q^{4}\right)\left(z^{2}-q^{12}\right)\left(z^{2}-q^{16}\right)\left(z^{2}-q^{24}\right), \\
& d_{2,4}(z)=\left(z^{2}+q^{8}\right)\left(z^{2}+q^{12}\right)\left(z^{2}+q^{16}\right)\left(z^{2}+q^{20}\right), \\
& d_{3,4}(z)=\left(z^{2}-q^{6}\right)^{\epsilon}\left(z^{2}-q^{10}\right)\left(z^{2}-q^{14}\right)^{2}\left(z^{2}-q^{18}\right)^{\epsilon}\left(z^{2}-q^{22}\right), \\
& d_{3,3}(z)=\left(z^{2}-q^{4}\right)\left(z^{2}-q^{8}\right)^{1+\epsilon}\left(z^{2}-q^{12}\right)^{2+\epsilon^{\prime}}\left(z^{2}-q^{16}\right)^{2+\epsilon}\left(z^{2}-q^{20}\right)^{2}\left(z^{2}-q^{24}\right), \\
& d_{2,3}(z)=\left(z^{2}+q^{6}\right)\left(z^{2}+q^{10}\right)^{1+\epsilon}\left(z^{2}+q^{14}\right)^{1+\epsilon}\left(z^{2}+q^{18}\right)^{2}\left(z^{2}+q^{22}\right), \\
& d_{1,3}(z)=\left(z^{2}+q^{8}\right)\left(z^{2}+q^{12}\right)\left(z^{2}+q^{16}\right)\left(z^{2}+q^{20}\right),
\end{aligned}
$$

for some $\epsilon, \epsilon^{\prime} \in\{0,1\}$.

4.2.2. $U_{q}^{\prime}\left(F_{4}^{(1)}\right)$. For type $F_{4}^{(1)}$, we have $q_{0}=q_{1}=q_{2}=q$ and $q_{3}=q_{4}=q^{1 / 2}$. Hence, we have $q_{s}=q^{1 / 2}$ and $p^{*}=q^{9}$. The classical decompositions of $V\left(\varpi_{1}\right)$ and $V\left(\varpi_{4}\right)$ as $U_{q}\left(\mathfrak{g}_{0}\right)$-modules are given as follows:

$$
V\left(\varpi_{1}\right) \simeq V\left(\bar{\Lambda}_{1}\right) \oplus V(0) \quad V\left(\varpi_{4}\right) \simeq V\left(\bar{\Lambda}_{4}\right) .
$$

For $V\left(\varpi_{1}\right)$ and $V\left(\varpi_{4}\right)$, we use the $U_{q}^{\prime}(\mathfrak{g})$-module structure given by Proposition 3.6. Note that for $V\left(\varpi_{4}\right)$, the corresponding $U_{q}\left(\mathfrak{g}_{0}\right)$-representation $V\left(\bar{\Lambda}_{4}\right)$ corresponds to the "little" adjoint representation as $\bar{\Lambda}_{4}$ is the highest short root. Hence the affine adjoint representation structure still applies.

By our SAGEMATH implementation, we can compute the denominator formulas $d_{1,1}(z), d_{1,4}(z)$ and $d_{1,4}(z)$ since we know their module structure (see Remark 4.3):

$$
\begin{aligned}
& d_{1,1}(z)=\left(z-q_{s}^{4}\right)\left(z-q_{s}^{10}\right)\left(z-q_{s}^{12}\right)\left(z-q_{s}^{18}\right), \\
& d_{1,4}(z)=\left(z+q_{s}^{8}\right)\left(z+q_{s}^{14}\right), \\
& d_{4,4}(z)=\left(z-q_{s}^{2}\right)\left(z-q_{s}^{8}\right)\left(z-q_{s}^{12}\right)\left(z-q_{s}^{18}\right) .
\end{aligned}
$$

Let us see $\mathbf{r}_{4,4}(x, y)$ more concretely. The $U_{q}\left(\mathfrak{g}_{0}\right)$-decomposition of $V\left(\varpi_{4}\right) \otimes V\left(\varpi_{4}\right)$ is given as follows:

$$
V\left(\varpi_{4}\right) \otimes V\left(\varpi_{4}\right) \simeq V\left(2 \bar{\Lambda}_{4}\right) \oplus V\left(\bar{\Lambda}_{3}\right) \oplus V\left(\bar{\Lambda}_{1}\right) \oplus V\left(\bar{\Lambda}_{4}\right) \oplus V(0)
$$

whose $U_{q}\left(\mathfrak{g}_{0}\right)$-highest weight vectors can be labeled as $u_{2 \bar{\Lambda}_{4}}, u_{\bar{\Lambda}_{4}}, u_{\bar{\Lambda}_{3}}, u_{\bar{\Lambda}_{1}}, u_{0}$. We can compute the following in $V\left(\varpi_{4}\right)_{x} \otimes V\left(\varpi_{4}\right)_{y}$, where we are considering an extension of scalars to $\mathbf{k}(x, y)$ :

$$
\begin{aligned}
e_{4} e_{3} e_{2} e_{1} e_{0} e_{*} u_{\bar{\Lambda}_{4}} & =\frac{[2]_{3}^{5}[2]_{4}^{4}[2]_{2}^{3}[2]_{1}^{2}[2]_{0}[3]_{3}^{2}\left(q_{s}^{4}+3 q_{s}^{2}+1\right)\left(q_{s}^{12} x-y\right)\left(q_{s}^{2} x-y\right)}{q_{s}^{4}(x y)^{4}} u_{2 \bar{\Lambda}_{4}}, \\
e_{*} u \bar{\Lambda}_{3} & =\frac{[2]_{3}^{5}[2]_{4}^{3}[2]_{2}^{3}[2]_{1}^{2}[2]_{0}[3]_{3}^{2}\left(q_{s}^{4}+3 q_{s}^{2}+1\right)\left(y-q_{s}^{2} x\right)}{q_{s}^{3}(x y)^{3}} u_{2 \bar{\Lambda}_{4}}, \\
e_{1} e_{2} e_{3}^{2} e_{2} e_{1} e_{0} u_{\bar{\Lambda}_{1}} & =[2]_{3} \frac{y-q_{s}^{8} x}{q_{s}^{2} x y} u_{2 \bar{\Lambda}_{4}}, \\
e_{0} e_{1} e_{2} e_{3}^{2} e_{2} e_{1} e_{0} u_{0} & =[2]_{3} \frac{\left(q_{s}^{18} x-y\right)\left(q_{s}^{8} x-y\right)}{q_{s}^{4}(x y)^{2}} u_{2 \bar{\Lambda}_{4}},
\end{aligned}
$$


where

$$
\begin{aligned}
& e_{*}=e_{3} e_{4} e_{2} e_{1} e_{3}^{2} e_{4} e_{2} e_{3} e_{2} e_{1} e_{3}^{2} e_{2}^{2} e_{1} e_{3} e_{4}^{2} e_{0} e_{3} e_{4} e_{2} e_{3}^{2} e_{1} e_{2}^{2} \\
& e_{1} e_{3} e_{4} e_{3} e_{4} e_{2} e_{3}^{2} e_{4} e_{2} e_{3} e_{1} e_{2} e_{3} e_{4} e_{0}^{2} e_{1}^{2} e_{2}^{2} e_{3}^{3} e_{2} e_{3} e_{1} e_{0} e_{2} e_{1} e_{0} .
\end{aligned}
$$

By $U_{q}^{\prime}\left(F_{4}^{(1)}\right)$-linearity and (4.28), we have

$$
\begin{array}{rlrl}
\mathbf{r}_{4,4}(x, y)\left(u_{2 \bar{\Lambda}_{4}}\right) & =a^{2 \bar{\Lambda}_{4}} u_{2 \bar{\Lambda}_{4}}, \quad & \mathbf{r}_{4,4}(x, y)\left(u_{\bar{\Lambda}_{3}}\right)=a^{\bar{\Lambda}_{3}} u_{\bar{\Lambda}_{3}}, & \mathbf{r}_{4,4}(x, y)\left(u_{\bar{\Lambda}_{1}}\right)=a^{\bar{\Lambda}_{1}} u_{\bar{\Lambda}_{1}}, \\
\mathbf{r}_{4,4}(x, y)\left(u_{\bar{\Lambda}_{4}}\right)=a^{\bar{\Lambda}_{4}} u_{\bar{\Lambda}_{4}}, & \mathbf{r}_{4,4}(x, y)\left(u_{0}\right)=a^{0} u_{0} .
\end{array}
$$

By using (4.30) and that $\mathbf{r}_{1,1}(x, y)$ is a $U_{q}^{\prime}(\mathfrak{g})$-isomorphism, we obtain the following.

Proposition 4.17. Put $z=x^{-1} y$ and denote $\mathbf{r}_{4,4}(z)=\mathbf{r}_{4,4}(x, y)$. Then we have

(1) $a^{2 \bar{\Lambda}_{4}}(z)=\left(z-q_{s}^{2}\right)\left(z-q_{s}^{8}\right)\left(z-q_{s}^{12}\right)\left(z-q_{s}^{18}\right)$,

(2) $a^{\bar{\Lambda}_{3}}(z)=\left(q_{s}^{2} z-1\right)\left(z-q_{s}^{8}\right)\left(z-q_{s}^{12}\right)\left(z-q_{s}^{18}\right)$,

(3) $a^{\bar{\Lambda}_{1}}(z)=\left(z-q_{s}^{2}\right)\left(q_{s}^{8} z-1\right)\left(z-q_{s}^{12}\right)\left(z-q_{s}^{18}\right)$,

(4) $a^{\bar{\Lambda}_{4}}(z)=\left(q_{s}^{2} z-1\right)\left(z-q_{s}^{8}\right)\left(q_{s}^{12} z-1\right)\left(z-q_{s}^{18}\right)$,

(5) $a^{0}(z)=\left(z-q_{s}^{2}\right)\left(z-q_{s}^{8}\right)\left(z-q_{s}^{12}\right)\left(q_{s}^{18} z-1\right)$.

Remark 4.18. By substituting $z$ with roots of $d_{4,4}(z)$ into $a(z)$ 's in Proposition 4.17, we can observe the following:

$$
\begin{aligned}
& \operatorname{Im}\left(\mathbf{r}_{4,4}\left(\left(-q_{s}\right)^{-6},\left(-q_{s}\right)^{6}\right)\right) \simeq V\left(\bar{\Lambda}_{4}\right) \text { as } U_{q}\left(F_{4}\right) \text {-modules, } \\
& \operatorname{Im}\left(\mathbf{r}_{4,4}\left(\left(-q_{s}\right)^{-4},\left(-q_{s}\right)^{4}\right)\right) \simeq V\left(\bar{\Lambda}_{1}\right) \oplus V(0) \text { as } U_{q}\left(F_{4}\right) \text {-modules. }
\end{aligned}
$$

Proposition 4.19. We have the following Dorey's type $U_{q}^{\prime}\left(F_{4}^{(1)}\right)$-homomorphisms:

$$
\begin{aligned}
V\left(\varpi_{1}\right)_{-q_{s}^{-2}} \otimes V\left(\varpi_{1}\right)_{-q_{s}^{2}} & \rightarrow V\left(\varpi_{2}\right), \\
V\left(\varpi_{4}\right)_{-q_{s}^{-1}} \otimes V\left(\varpi_{4}\right)_{-q_{s}^{1}} & \rightarrow V\left(\varpi_{3}\right), \\
V\left(\varpi_{4}\right)_{q_{s}^{-6}} \otimes V\left(\varpi_{4}\right)_{q_{s}^{6}} & \rightarrow V\left(\varpi_{4}\right), \\
V\left(\varpi_{4}\right)_{q_{s}^{-4}} \otimes V\left(\varpi_{4}\right)_{q_{s}^{4}} & \rightarrow V\left(\varpi_{1}\right)_{-1} .
\end{aligned}
$$

Proof. Note that (4.32a) and (4.32b) are known as T-system ([Her06, Section 7]). By Proposition 4.17 and (4.31a), (3.1) implies that there exists a Dorey's type homomorphism

$$
V\left(\varpi_{4}\right)_{q_{s}^{-6}} \otimes V\left(\varpi_{4}\right)_{q_{s}^{6}} \rightarrow V\left(\varpi_{4}\right)_{a}
$$

for $a \in\{1,-1\}$.

If $a=-1$, then $d_{4,4}(z)$ has a root $-q_{s}^{12}$ by taking the dual of (4.33):

$$
V\left(\varpi_{4}\right)_{q_{s}^{-6}} \hookrightarrow V\left(\varpi_{4}\right)_{-1} \otimes V\left(\varpi_{4}\right)_{q_{s}^{-12}} .
$$

However, this can not happen by (4.29a). Similarly, once can check (4.32d).

Using our SAGEMATH implementation, we also have that

$$
\mathbf{r}_{1,1}(x, y)\left(u_{\bar{\Lambda}_{2}}^{\prime}\right)=\left(q_{s}^{4} z-1\right)\left(z-q_{s}^{10}\right)\left(z-q_{s}^{12}\right)\left(z-q_{s}^{18}\right) u_{\bar{\Lambda}_{2}}^{\prime},
$$

where $u_{\bar{\Lambda}_{2}}^{\prime}$ is the $U_{q}\left(\mathfrak{g}_{0}\right)$-highest weight vector in the $U_{q}\left(\mathfrak{g}_{0}\right)$-decomposition

$$
V\left(\varpi_{1}\right) \otimes V\left(\varpi_{1}\right) \simeq V\left(2 \bar{\Lambda}_{1}\right) \oplus V\left(\bar{\Lambda}_{2}\right) \oplus V\left(2 \bar{\Lambda}_{4}\right) \oplus V\left(\bar{\Lambda}_{1}\right)^{\oplus 3} \oplus V(0)^{\oplus 2} .
$$


Using Proposition 4.17 and (4.34), we can compute

$$
\begin{aligned}
& R_{4,4}^{\text {norm }}\left(u \otimes f_{4} u\right)=\frac{\left(1-q_{s}^{2}\right)}{\left(z-q_{s}^{2}\right)}\left(u \otimes f_{4} u\right)+\frac{q_{s}(z-1)}{\left(z-q_{s}^{2}\right)}\left(f_{4} u \otimes u\right), \\
& R_{1,1}^{\text {norm }}\left(u \otimes f_{1} u\right)=\frac{\left(1-q_{s}^{4}\right)}{\left(z-q_{s}^{4}\right)}\left(u \otimes f_{1} u\right)+\frac{q_{s}^{2}(z-1)}{\left(z-q_{s}^{4}\right)}\left(f_{1} u \otimes u\right) .
\end{aligned}
$$

Now we can compute all $a_{i, j}(z)$ 's clearly, and compute $d_{i, j}(z)$ 's with some ambiguities (will be all refined later) by using the same arguments in previous subsections. We record here the $d_{i, j}(z)$ 's:

$$
\begin{aligned}
& d_{1,2}(z)=\left(z+q_{s}^{6}\right)\left(z+q_{s}^{8}\right)\left(z+q_{s}^{10}\right)\left(z+q_{s}^{12}\right)\left(z+q_{s}^{14}\right)\left(z+q_{s}^{16}\right), \\
& d_{1,3}(z)=\left(z-q_{s}^{7}\right)\left(z-q_{s}^{9}\right)\left(z-q_{s}^{13}\right)\left(z-q_{s}^{15}\right), \\
& d_{2,2}(z)=\left(z-q_{s}^{4}\right)\left(z-q_{s}^{6}\right)\left(z-q_{s}^{8}\right)^{\epsilon}\left(z-q_{s}^{10}\right)^{\epsilon}\left(z-q_{s}^{12}\right)^{2}\left(z-q_{s}^{14}\right)^{2}\left(z-q_{s}^{16}\right)\left(z-q_{s}^{18}\right), \\
& d_{2,3}(z)=\left(z+q_{s}^{5}\right)\left(z+q_{s}^{7}\right)^{\epsilon}\left(z+q_{s}^{9}\right)\left(z+q_{s}^{11}\right)^{\epsilon+1}\left(z+q_{s}^{13}\right)\left(z+q_{s}^{15}\right)\left(z+q_{s}^{17}\right), \\
& d_{2,4}(z)=\left(z-q_{s}^{6}\right)\left(z-q_{s}^{10}\right)\left(z-q_{s}^{12}\right)\left(z-q_{s}^{16}\right), \\
& d_{3,3}(z)=\left(z-q_{s}^{2}\right)\left(z-q_{s}^{6}\right)\left(z-q_{s}^{8}\right)^{1}\left(z-q_{s}^{10}\right)^{1}\left(z-q_{s}^{12}\right)^{2}\left(z-q_{s}^{16}\right)\left(z-q_{s}^{18}\right), \\
& d_{3,4}(z)=\left(z+q_{s}^{3}\right)\left(z+q_{s}^{7}\right)\left(z+q_{s}^{9}\right)^{\epsilon^{\prime}}\left(z+q_{s}^{11}\right)\left(z+q_{s}^{13}\right)\left(z+q_{s}^{17}\right) .
\end{aligned}
$$

for some $\epsilon, \epsilon^{\prime} \in\{0,1\}$. By our SageMath implementation, we have shown that $\epsilon^{\prime}=0$ in (4.36g). Therefore, we can obtain $d_{3,3}(z)$ in (4.36f).

4.3. $U_{q}^{\prime}\left(E_{6,7,8}^{(1)}\right)$. Comparing with the quantum affine algebras have dealt with in the previous subsections, the categories $\mathcal{C}_{Q}$ for $U_{q}^{\prime}\left(E_{6,7,8}^{(1)}\right)$ are relatively well-understood (see [HL10, Oh19]). For instance, we know Dorey's rule.

For a while, we shall review another statistics on $\Gamma_{Q}$.

Definition 4.20. Let $Q$ be a Dynkin quiver of type $A_{n}, D_{n}$ or $E_{6,7,8}$. For pairs $\underline{p}^{\prime}=\left(\alpha^{(1)}, \beta^{(1)}\right) \prec_{[Q]}^{\mathrm{b}}$ $\underline{p}=\left(\alpha^{(2)}, \beta^{(2)}\right)$ in $\mathbb{Z}_{\geq 0}^{\mathrm{N}}$, we say that they are good adjacent neighbors if

(i) there exists $\eta \in \Phi^{+}$satisfying one of the following conditions:

(a) $\eta+\beta^{(2)}=\beta^{(1)}, \eta+\alpha^{(1)}=\alpha^{(2)}$ and $\operatorname{dist}_{[Q]}\left(\eta, \beta^{(2)}\right), \operatorname{dist}_{[Q]}\left(\eta, \alpha^{(1)}\right)<\operatorname{dist}_{[Q]}(\underline{p})$,

(b) $\beta^{(1)}+\eta=\beta^{(2)}, \alpha^{(2)}+\eta=\alpha^{(1)}$ and $\operatorname{dist}_{[Q]}\left(\beta^{(1)}, \eta\right), \operatorname{dist}_{[Q]}\left(\alpha^{(2)}, \eta\right)<\operatorname{dist}_{[Q]}(\underline{p})$,

(ii) there exists no $\underline{p}^{\prime \prime} \prec_{[Q]}^{\mathrm{b}} \underline{p}$ such that it satisfies the conditions (i) or

$$
\underline{p}^{\prime} \prec_{[Q]}^{\mathrm{b}} \underline{p}^{\prime \prime} \prec_{[Q]}^{\mathrm{b}} \underline{p} .
$$

Definition 4.21. For a non $[Q]$-simple pair $\underline{p}$, the $[Q]$-length of the pair $\underline{p} \in \mathbb{Z}_{>0}^{N}$, denoted by $\operatorname{len}_{[Q]}(\underline{p})$, is the integer which counts the number of all non $[Q]$-simple pairs $\underline{p}^{\prime} \in \mathbb{Z}_{\geq 0}^{\mathrm{N}}$ satisfying the following property:

- $\underline{p}^{\prime} \prec_{[Q]}^{\mathrm{b}} \underline{p}$ and there exists a sequence of pairs

$$
\underline{p}^{(0)}=\underline{p}^{\prime} \prec_{[Q]}^{\mathrm{b}} \underline{p}^{(1)} \prec_{[Q]}^{\mathrm{b}} \underline{p}^{(2)} \prec_{[Q]}^{\mathrm{b}} \cdots \prec_{[Q]}^{\mathrm{b}} \underline{p}^{(k)}=\underline{p} \quad\left(k \in \mathbb{Z}_{\geq 1}\right)
$$

such that $\underline{p}^{(i)}, \underline{p}^{(i+1)}$ are good adjacent neighbor for all $0 \leq i \leq k-1$.

We call the pairs $\underline{p}^{\prime}, \underline{p}$ good neighbors. 
Proposition 4.22 ([Oh19, Theorem $6.9(3)])$. For a $[Q]$-simple pair $\underline{p}=(\alpha, \beta), V_{Q}(\alpha) \otimes V_{Q}(\beta)$ is simple.

Corollary 4.23. For a pair $\underline{p}=(\alpha, \beta)$ with $\widehat{\Omega}_{Q}(\alpha)=(i, p)$ and $\widehat{\Omega}_{Q}(\beta)=\left(j, p^{\prime}\right),(-q)^{\left|p-p^{\prime}\right|}$ is not a root of $d_{i, j}(z)$ if and only if $\underline{p}$ is $[Q]$-simple.

Proof. Our assertion follows from Theorem 3.10 and Proposition 4.22.

Proposition 4.24 ([Oh19, Theorem 5.23]). For a non $[Q]$-simple pair $\underline{p}=(\alpha, \beta)$, the composition length of $V_{Q}(\alpha) \otimes V_{Q}(\beta)$ is larger than or equal to $\operatorname{len}_{[Q]}(\underline{p})+2$.

Corollary 4.25. For a non $[Q]$-simple pair $\underline{p}=(\alpha, \beta)$ with $\operatorname{len}_{[Q]}(\underline{p}) \geq 3$, the order of root $(-q)^{\left|p-p^{\prime}\right|}$ of $d_{i, j}(z)$ is strictly larger than 1 , when $\widehat{\Omega}_{Q}(\alpha)=(i, p)$ and $\widehat{\Omega}_{Q}(\beta)=\left(j, p^{\prime}\right)$.

Proof. Our assertion follows from Lemma 3.7 and Proposition 4.24.

Remark 4.26. Using Corollary 4.23 and 4.25 , we can determine whether the give root $(-q)^{\left|p-p^{\prime}\right|}$ of $d_{i, j}(z)$ is one or larger than one, by observing the composition length. Here, $(-q)^{\left|p-p^{\prime}\right|}$ arises from a non $[Q]$-simple pair $(\alpha, \beta)$ with $\widehat{\Omega}_{Q}(\alpha)=(i, p)$ and $\widehat{\Omega}_{Q}(\beta)=\left(j, p^{\prime}\right)$.

For $U_{q}^{\prime}\left(E_{6}^{(1)}\right), U_{q}^{\prime}\left(E_{7}^{(1)}\right)$ and $U_{q}^{\prime}\left(E_{8}^{(1)}\right)$, we shall use the $\Gamma_{Q}$ 's given in (2.6), Appendix A, and Appendix B, respectively, to apply Corollary 4.23 and 4.25 .

4.3.1. $E_{6}^{(1)}$. In our SAGEMATh implementation, we use the $U_{q}^{\prime}(\mathfrak{g})$-module structure on $V\left(\varpi_{1}\right)$ and $V\left(\varpi_{6}\right)$ given by Proposition 3.3 and $V\left(\varpi_{2}\right)$ given by Proposition 3.6. Therefore, we obtain denominator formulas for $d_{1,1}(z)=d_{6,6}(z), d_{1,6}(z), d_{1,2}(z)=d_{2,6}(z)$ and $d_{2,2}(z)$ as follows:

$$
\begin{array}{ll}
d_{1,1}(z)=d_{6,6}(z)=\left(z-q^{2}\right)\left(z-q^{8}\right), & d_{1,6}(z)=\left(z-q^{6}\right)\left(z-q^{12}\right), \\
d_{1,2}(z)=d_{2,6}(z)=\left(z+q^{5}\right)\left(z+q^{9}\right), & d_{2,2}(z)=\left(z-q^{2}\right)\left(z-q^{6}\right)\left(z-q^{8}\right)\left(z-q^{12}\right) .
\end{array}
$$

Additionally, we can obtain the following computation by our SAGEMATH implementation: For $i=1,2$ or 6 , we have

$$
R_{i, i}^{\text {norm }}\left(u_{\varpi_{i}} \otimes f_{i}\left(u_{\varpi_{i}}\right)_{z}\right)=\frac{1-q^{2}}{z-q^{2}}\left(u_{\varpi_{i}}\right)_{z} \otimes f_{i} u_{\varpi_{i}}+\frac{q(z-1)}{z-q^{2}} f_{i}\left(u_{\varpi_{i}}\right)_{z} \otimes u_{\varpi_{i}} .
$$

The Dorey's type morphism

$$
V\left(\varpi_{1}\right)_{-q^{-1}} \otimes V\left(\varpi_{1}\right)_{-q} \rightarrow V\left(\varpi_{3}\right)
$$

given by Theorem 3.13 can be observed using $\Gamma_{Q}$ given in (2.6).

Proposition 4.27. We have $d_{1,3}(z)=d_{3,6}(z)=\left(z+q^{3}\right)\left(z+q^{7}\right)\left(z+q^{9}\right)$.

Proof. By applying the technique have applied, we have

$$
a_{1,1}(z) \equiv \frac{[6][18][24][0]}{[2][8][16][22]}, \quad a_{1,3}(z) \equiv \frac{[1][5][19][23]}{[3][9][15][21]}
$$

where $[a]:=\left((-q)^{a} z ; q^{24}\right)$. Applying Lemma 3.8 to (4.38) with $W=V\left(\varpi_{1}\right)$, we have

$$
\frac{\left(z+q^{-1}\right)\left(z+q^{3}\right)\left(z+q^{7}\right)\left(z+q^{9}\right)}{d_{1,3}(z)} \in \mathbf{k}\left[z^{ \pm 1}\right] .
$$


By Corollary 4.25, we can observe $\left(\left(\begin{array}{c}000 \\ 110\end{array}\right),\left(\begin{array}{c}000 \\ 001\end{array}\right)\right),\left(\left(\begin{array}{l}112 \\ 221\end{array}\right),\left(\begin{array}{c}000 \\ 001\end{array}\right)\right)$ and $\left(\left(\begin{array}{l}111 \\ 221\end{array}\right),\left(\begin{array}{c}000 \\ 001\end{array}\right)\right)$ in $\Gamma_{Q}$ are minimal pairs of $[Q]$-simples $\left(\left(\begin{array}{c}000 \\ 111\end{array}\right)\right),\left(\left(\begin{array}{c}011 \\ 111\end{array}\right),\left(\begin{array}{l}101 \\ 111\end{array}\right)\right)$ and $\left(\left(\begin{array}{c}111 \\ 211\end{array}\right)\right)$, respectively. Thus Corollary 4.23 and Corollary 4.25 tell that $\left(z+q^{3}\right)\left(z+q^{7}\right)\left(z+q^{9}\right)$ divides $d_{1,3}(z)$. Since $d_{1,3}(z)$ has roots in $\mathbb{C}\left[\left[q^{1 / m}\right]\right] q^{1 / m}$ for some $m \in \mathbb{Z}_{>0}$, we have

$$
d_{1,3}(z)=\left(z+q^{3}\right)\left(z+q^{7}\right)\left(z+q^{9}\right)
$$

as we desired.

Proposition 4.28. We have

$$
d_{3,3}(z)=d_{5,5}(z)=\left(z-q^{2}\right)\left(z-q^{4}\right)\left(z-q^{6}\right)\left(z-q^{8}\right)^{2}\left(z-q^{10}\right) .
$$

Proof. Applying Lemma 3.8 to (4.38) with $W=V\left(\varpi_{3}\right)$, we have

$$
\begin{aligned}
& a_{3,3}(z) \equiv a_{1,3}(-q z) a_{1,3}\left(-q^{-1} z\right)=\frac{[0][6][18][24]}{[8][10][14][16]}, \\
& \frac{\left(z-q^{2}\right)\left(z-q^{4}\right)\left(z-q^{6}\right)\left(z-q^{8}\right)^{2}\left(z-q^{10}\right)}{d_{3,3}(z)} \in \mathbf{k}\left[z^{ \pm 1}\right] .
\end{aligned}
$$

As in the previous proposition, one can check that $\left(z-q^{2}\right)\left(z-q^{4}\right)\left(z-q^{6}\right)\left(z-q^{8}\right)\left(z-q^{10}\right)$ divides $d_{3,3}(z)$. Furthermore, for the pair $\underline{p}=\left(\left(\begin{array}{l}111 \\ 210\end{array}\right),\left(\begin{array}{c}000 \\ 011\end{array}\right)\right)$, we can check

$$
\left.\begin{array}{l}
\underline{p}^{(1)}=\left(\left(\begin{array}{c}
101 \\
110
\end{array}\right),\left(\begin{array}{c}
010 \\
111
\end{array}\right)\right.
\end{array}\right) \prec_{Q}^{\mathrm{b}} \underline{p}=\left(\left(\begin{array}{l}
111 \\
210
\end{array}\right),\left(\begin{array}{c}
000 \\
011
\end{array}\right)\right)
$$

and $\underline{p}^{(i)}(i=1,2)$ and $\underline{p}$ are good adjacent neighbors. Thus $\left(z-q^{8}\right)^{2}$ divides $d_{3,3}(z)$, which yields our assertion.

Proposition 4.29. We have

$$
d_{4,4}(z)=\left(z-q^{2}\right)\left(z-q^{4}\right)^{2}\left(z-q^{6}\right)^{2+\epsilon}\left(z-q^{8}\right)^{3}\left(z-q^{10}\right)^{2}\left(z-q^{12}\right),
$$

for some $\epsilon \in\{0,1\}$.

Proof. Applying Lemma 3.8 to

$$
V\left(\varpi_{3}\right)_{-q^{-1}} \otimes V\left(\varpi_{1}\right)_{q^{2}} \rightarrow V\left(\varpi_{4}\right)
$$

with $W=V\left(\varpi_{3}\right)$, we have

$$
\begin{aligned}
& a_{4,4}(z) \equiv a_{3,4}\left(-q^{-1} z\right) a_{1,4}\left(q^{2} z\right)=\frac{[0][2][4][20][22][24]}{[8][10][12]^{2}[14][16]} \\
& \frac{\left(z-q^{2}\right)\left(z-q^{4}\right)^{2}\left(z-q^{6}\right)^{3}\left(z-q^{8}\right)^{3}\left(z-q^{10}\right)^{2}\left(z-q^{12}\right)}{d_{4,4}(z)} \in \mathbf{k}\left[z^{ \pm 1}\right] .
\end{aligned}
$$

As in the previous proposition, we can check

$$
\frac{d_{4,4}(z)}{\left(z-q^{2}\right)\left(z-q^{4}\right)^{2}\left(z-q^{6}\right)^{2}\left(z-q^{8}\right)^{2}\left(z-q^{10}\right)^{2}\left(z-q^{12}\right)} \in \mathbf{k}\left[z^{ \pm 1}\right] .
$$

If the multiplicity of $q^{8}$ is 2, we have contradiction for (3.8) and (4.41). Hence our assertion follows. 
Applying the techniques in Proposition 4.27 and Proposition 4.28, we can obtain the $d_{i, j}(z)$ 's:

$$
\begin{aligned}
& d_{1,4}(z)=d_{4,6}(z)=\left(z-q^{4}\right)\left(z-q^{6}\right)\left(z-q^{8}\right)\left(z-q^{10}\right), \\
& d_{1,5}(z)=d_{3,6}(z)=\left(z+q^{5}\right)\left(z+q^{7}\right)\left(z+q^{11}\right), \\
& d_{3,4}(z)=d_{4,5}(z)=\left(z+q^{3}\right)\left(z+q^{5}\right)^{2}\left(z+q^{7}\right)^{2}\left(z+q^{9}\right)^{2}\left(z+q^{11}\right), \\
& d_{3,5}(z)=\left(z-q^{4}\right)\left(z-q^{6}\right)^{2}\left(z-q^{8}\right)\left(z-q^{10}\right)\left(z-q^{12}\right), \\
& d_{3,2}(z)=d_{2,5}(z)=\left(z-q^{4}\right)\left(z-q^{6}\right)\left(z-q^{8}\right)\left(z-q^{10}\right), \\
& d_{4,4}(z)=\left(z-q^{2}\right)\left(z-q^{4}\right)^{2}\left(z-q^{6}\right)^{2+\epsilon}\left(z-q^{8}\right)^{3}\left(z-q^{10}\right)^{2}\left(z-q^{12}\right), \\
& d_{2,4}(z)=\left(z+q^{3}\right)\left(z+q^{5}\right)\left(z+q^{7}\right)^{2}\left(z+q^{9}\right)\left(z+q^{11}\right) .
\end{aligned}
$$

The $\epsilon$ is indeed 1, which is proved in an addendum to our publication [OS19] (see Appendix E).

4.3.2. $E_{7}^{(1)}$. Using our SAgEMATH implementation for $V\left(\varpi_{1}\right)$ and $V\left(\varpi_{7}\right)$ with the $U_{q}^{\prime}(\mathfrak{g})$-module structure by Proposition 3.3, we can obtain denominator formulas for $d_{1,1}(z), d_{1,7}(z), d_{7,7}(z)$ as follows:

$$
\begin{aligned}
& d_{1,1}(z)=\left(z-q^{2}\right)\left(z-q^{8}\right)\left(z-q^{12}\right)\left(z-q^{18}\right), \\
& d_{1,7}(z)=\left(z+q^{7}\right)\left(z+q^{13}\right), \\
& d_{7,7}(z)=\left(z-q^{2}\right)\left(z-q^{10}\right)\left(z-q^{18}\right) .
\end{aligned}
$$

Using the Dorey's type morphisms obtained from Theorem 3.13, we can compute $d_{i, j}(z)$ 's almost completely. See Appendix C, as in the $E_{6}^{(1)}$ case.

4.3.3. $E_{8}^{(1)}$. By our SAGEMATH implementation using the $U_{q}^{\prime}(\mathfrak{g})$-module structure on $V\left(\varpi_{8}\right)$ given by Proposition 3.6, we can obtain denominator formulas for $d_{8,8}(z)$ as follows:

$$
d_{8,8}(z)=\left(z-q^{2}\right)\left(z-q^{12}\right)\left(z-q^{20}\right)\left(z-q^{30}\right) .
$$

Moreover, using the Dorey's type surjection

$$
V\left(\varpi_{8}\right)_{q^{-6}} \otimes V\left(\varpi_{8}\right)_{q^{6}} \rightarrow V\left(\varpi_{1}\right)
$$

to define the $U_{q}^{\prime}(\mathfrak{g})$-module structure of $V\left(\varpi_{1}\right)$, we can use our SAGEMATH implementation to compute

$$
d_{1,1}(z)=\left(z-q^{2}\right)\left(z-q^{8}\right)\left(z-q^{12}\right)\left(z-q^{14}\right)\left(z-q^{18}\right)\left(z-q^{20}\right)\left(z-q^{24}\right)\left(z-q^{30}\right) .
$$

Note that for $i=1,8$, there is a unique $j \sim i$, and that

$$
\begin{aligned}
u_{\bar{\Lambda}_{j}} & =u_{\varpi_{i}} \otimes f_{i} u_{\varpi_{i}}-q u_{\varpi_{i}} \otimes f_{i} u_{\varpi_{i}}, \\
\frac{\mathbf{r}_{i, i}(x, y)\left(u_{\bar{\Lambda}_{j}}\right)}{d_{i, i}(z)} & =\frac{q^{2} z-1}{z-q^{2}} u_{\bar{\Lambda}_{j}},
\end{aligned}
$$

the former is the $U_{q}\left(\mathfrak{g}_{0}\right)$-highest weight vector in $V\left(\varpi_{i}\right) \otimes V\left(\varpi_{i}\right)$ and the latter of which is computed by our SageMath implementation. Thus, we perform the following computations:

$$
R_{8,8}^{\text {norm }}\left(u_{\varpi_{8}} \otimes f_{8}\left(u_{\varpi_{8}}\right)_{z}\right)=\frac{1-q^{2}}{z-q^{2}}\left(u_{\varpi_{8}}\right)_{z} \otimes f_{8} u_{\varpi_{8}}+\frac{q(z-1)}{z-q^{2}} f_{8}\left(u_{\varpi_{8}}\right)_{z} \otimes u_{\varpi_{8}},
$$




$$
\begin{aligned}
& R_{1,1}^{\text {norm }}\left(u_{\varpi_{1}} \otimes f_{1}\left(u_{\varpi_{1}}\right)_{z}\right)=\frac{1-q^{2}}{z-q^{2}}\left(u_{\varpi_{1}}\right)_{z} \otimes f_{1} u_{\varpi_{1}}+\frac{q(z-1)}{z-q^{2}} f_{1}\left(u_{\varpi_{1}}\right)_{z} \otimes u_{\varpi_{1}}, \\
& R_{8,8}^{\text {norm }}(z)\left(u_{\varpi_{8}} \otimes f_{*} u_{\varpi_{8}}\right)=\frac{\left(z-q^{10}\right)(z-1)}{\left(z-q^{2}\right)\left(z-q^{12}\right)} f_{*} u \otimes u+\text { other terms, }
\end{aligned}
$$

where $f_{*}=f_{8} f_{7} f_{6} f_{5} f_{4} f_{2} f_{3} f_{4} f_{5} f_{6} f_{7} f_{8}$. We note that we used our SAGEMATH implementation to compute the last one, but it should be possible to compute by hand using $\mathbf{r}_{8,8}(x, y)$ on classically highest weight vectors.

Remark 4.30. We note that all computations of $\mathbf{r}_{i, i}\left(u_{\varpi_{i}} \otimes f_{i} u_{\varpi_{i}}\right)$ for degree 1 vertices $i$ in the Dynkin diagram all have the same form of (4.45). This is reflected in the fact that, e.g., (4.46a) and (4.46b) are also of the same form as (4.37). Thus, there appears to be some common structure among all of these modules and $R$-matrices.

Using the Dorey's type morphisms obtained from Theorem 3.13 with the above computations, we can compute $d_{i, j}(z)$ 's almost completely. See Appendix D.

4.4. Conclusion: Folded AR quiver and denominator formulas. Now we can conclude that the denominator formulas for exceptional types can be read for their corresponding folded AR quivers in the following sense:

\section{Theorem 4.31.}

(1) $V\left(\varpi_{i}\right)_{p} \otimes V\left(\varpi_{j}\right)_{p^{\prime}}$ is reducible if and only if there exists a folded AR quiver $\widehat{\Upsilon}_{\left[\widetilde{w}_{0}\right]}$ and a pair $(\alpha, \beta)$ such that

(i) $(\alpha, \beta)$ is non $\left[\widetilde{\mathrm{w}}_{0}\right]$-simple,

(ii) $V\left(\varpi_{i}\right)_{p} \simeq V_{\left[\widetilde{\mathrm{w}}_{0}\right]}(\alpha)_{a}$ and $V\left(\varpi_{j}\right)_{p^{\prime}} \simeq V_{\left[\widetilde{\mathrm{w}}_{0}\right]}(\beta)_{a}$ for some $a \in \mathbf{k}^{\times}$,

(2) $\pm q^{t / \mathrm{d}}$ is a root of $d_{i, j}(z)$ of order larger than equal to 2 if and only if $\theta_{t}^{\llbracket \widetilde{\mathrm{w}}_{0} \rrbracket}(i, j) \geq 2$. In particular, if $(\alpha, \beta)$ is a $\left[\widetilde{\mathrm{w}}_{0}\right]$-minimal pair of $\alpha+\beta \in \Phi^{+}$and contained in $\Phi_{\left[\widetilde{\mathrm{w}}_{0}\right]}(i, j)[t]$ for some $\left[\widetilde{\mathrm{w}}_{0}\right]$, then $\pm q^{t / \mathrm{d}}$ is a root of $d_{i, j}(z)$ of order 1 .

Furthermore, when $\mathfrak{g}$ is of type $G_{2}^{(1)}$ and $F_{4}^{(1)}$,

$$
d_{i, j}^{\mathfrak{g}}(z)=D_{i, j}^{\llbracket \widetilde{\mathrm{w}}_{0} \rrbracket}\left(z ;-q^{1 / \mathrm{d}}\right) \times\left(z-p^{*}\right)^{\delta_{i, j}} .
$$

We have seen that the denominator formulas for $U_{q}^{\prime}\left(D_{4}^{(3)}\right)$ can be obtained from the ones for $U_{q}\left(D_{4}^{(1)}\right)$ (Corollary 4.11) and hence $U_{q}\left(D_{4}^{(2)}\right)$ (Theorem 3.11). The following can be understood as a $E_{6}$-analogues: We can observe (up to \pm 1 )

$$
\begin{aligned}
& d_{1,1}^{E_{6}^{(2)}}(z)=d_{1,1}^{E_{6}^{(1)}}(z) d_{1,6}^{E_{6}^{(1)}}(-z), d_{1,2}^{E_{6}^{(2)}}(z)=d_{1,3}^{E_{6}^{(1)}}(z) d_{1,5}^{E_{6}^{(1)}}(-z), d_{4,4}^{E_{6}^{(2)}}(z)=d_{2,2}^{E_{6}^{(1)}}(z) d_{2,2}^{E_{6}^{(1)}}(-z), \\
& d_{2,2}^{E_{6}^{(2)}}(z)=d_{3,3}^{E_{6}^{(1)}}(z) d_{3,5}^{E_{6}^{(1)}}(-z), d_{3,4}^{E_{6}^{(2)}}(z)=d_{2,4}^{E_{6}^{(1)}}(z) d_{2,4}^{E_{6}^{(1)}}(-z), \\
& d_{1,3}^{E_{6}^{(2)}}(z)=d_{1,4}^{E_{6}^{(1)}}(\sqrt{-1} z) d_{1,4}^{E_{6}^{(1)}}(-\sqrt{-1} z), d_{1,4}^{E_{6}^{(2)}}(z)=d_{1,2}^{E_{6}^{(1)}}(\sqrt{-1} z) d_{1,2}^{E_{6}^{(1)}}(-\sqrt{-1} z), \\
& d_{2,4}^{E_{6}^{(2)}}(z)=d_{2,3}^{E_{6}^{(1)}}(\sqrt{-1} z) d_{2,3}^{E_{6}^{(1)}}(-\sqrt{-1} z), d_{2,3}^{E_{6}^{(2)}}(z)=d_{3,4}^{E_{6}^{(1)}}(\sqrt{-1} z) d_{3,4}^{E_{6}^{(1)}}(-\sqrt{-1} z),
\end{aligned}
$$

and we expect

$$
d_{3,3}^{E_{6}^{(2)}}(z)=d_{4,4}^{E_{6}^{(1)}}(z) d_{4,4}^{E_{6}^{(1)}}(-z)
$$


Remark 4.32. In (4.43f), we have shown the existence of a root of $d_{4,4}(z)$ whose order is 3 . Such roots of order at least 3 can also be found in the denominator formulas for $E_{7}^{(1)}$ and $E_{8}^{(1)}$ in Appendix C and Appendix D respectively. To the best knowledge of the authors, this is the first such observation of a root of order strictly larger than 2 .

\section{Quiver Hecke algebras and generalized Schur-Weyl Duality}

In this section, we shall review the representation theory of quiver Hecke algebras, introduced by Khovanov-Lauda and Rouquier independently, and generalized Schur-Weyl duality constructed in [KKK18, KKK15].

5.1. Quiver Hecke algebras. We recall the definition of quiver Hecke algebra associated with a symmetrizable Cartan datum $\left(\mathrm{A}, P, \Pi, P^{\vee}, \Pi^{\vee},(\cdot, \cdot)\right)$ satisfying $\left(\alpha_{i}, \alpha_{i}\right) \in 2 \mathbb{Z}$ for all $i \in I$. Let us take a family of polynomials $\left(Q_{i, j}\right)_{i, j \in I}$ in $\mathbf{k}[u, v]$ satisfying the following condition:

$$
Q_{i, j}(u, v)=\left(1-\delta_{i, j}\right) \sum_{\substack{p . q \in \mathbb{Z}_{\geq 0} \\\left(\alpha_{i}, \alpha_{i}\right) p+\left(\alpha_{j}, \alpha_{j}\right) q=-2\left(\alpha_{i}, \alpha_{j}\right)}} t_{i, j ; p, q} u^{p} v^{q},
$$

with $t_{i, j ; p, q} \in \mathbf{k}, t_{i, j ; p, q}=t_{j, i ; q, p}$ and $t_{i, j ;-a_{i j}, 0} \in \mathbf{k}^{\times}$. Then we have

$$
Q_{i, j}(u, v)=Q_{j, i}(v, u) \quad \text { for all } i, j \in I .
$$

We denote by $\mathfrak{S}_{n}=\left\langle s_{i} \mid 1 \leq i \leq n\right\rangle$ the symmetric group on $n$-letters, where $s_{i}$ denote the transposition $(i, i+1)$ of $i$ and $i+1$. Then $\mathfrak{S}_{n}$ acts on $I^{n}$ by place permutations.

For $\beta \in \mathrm{Q}^{+}$with $|\beta|=n$, we set

$$
I^{\beta}=\left\{\nu=\left(\nu_{1}, \ldots, \nu_{n}\right) \in I^{n} \mid \alpha_{\nu_{1}}+\cdots+\alpha_{\nu_{n}}=\beta\right\} .
$$

Definition 5.1 ([KL09, KL11, Rou08]). For $\beta \in \mathrm{Q}^{+}$with $|\beta|=n$, the quiver Hecke algebra $R(\beta)$ at $\beta$ associated with a Cartan datum $\left(\mathrm{A}, P, \Pi, P^{\vee}, \Pi^{\vee},(\cdot, \cdot)\right)$ and a matrix $\left(Q_{i, j}\right)_{i, j \in I}$ is the k-algebra generated by the elements $\{e(\nu)\}_{\nu \in I^{\beta}},\left\{x_{k}\right\}_{1 \leq k \leq n},\left\{\tau_{m}\right\}_{1 \leq m \leq n-1}$ satisfying the following defining relations:

$$
\begin{aligned}
& e(\nu) e\left(\nu^{\prime}\right)=\delta_{\nu, \nu^{\prime}} e(\nu), \quad \sum_{\nu \in I^{\beta}} e(\nu)=1, \quad x_{k} x_{m}=x_{m} x_{k}, \quad x_{k} e(\nu)=e(\nu) x_{k}, \\
& \tau_{m} e(\nu)=e\left(s_{m}(\nu)\right) \tau_{m}, \quad \tau_{k} \tau_{m}=\tau_{m} \tau_{k} \quad \text { if }|k-m|>1, \quad \tau_{k}^{2} e(\nu)=Q_{\nu_{k}, \nu_{k+1}}\left(x_{k}, x_{k+1}\right) e(\nu), \\
& \left(\tau_{k} x_{m}-x_{s_{k}(m)} \tau_{k}\right) e(\nu)= \begin{cases}-e(\nu) & \text { if } m=k, \nu_{k}=\nu_{k+1}, \\
e(\nu) & \text { if } m=k+1, \nu_{k}=\nu_{k+1}, \\
0 & \text { otherwise, }\end{cases} \\
& \left(\tau_{k+1} \tau_{k} \tau_{k+1}-\tau_{k} \tau_{k+1} \tau_{k}\right) e(\nu)=\delta_{\nu_{k}, \nu_{k+2}} \frac{Q_{\nu_{k}, \nu_{k+1}}\left(x_{k}, x_{k+1}\right)-Q_{\nu_{k}, \nu_{k+1}}\left(x_{k+2}, x_{k+1}\right)}{x_{k}-x_{k+2}} e(\nu) .
\end{aligned}
$$

Let us denote by $\operatorname{Mod}(R(\beta))$ the category of $R(\beta)$-modules and by $\operatorname{Mod}_{\mathrm{gr}}(R(\beta))$ the category of graded $R(\beta)$-modules. The category of graded $R(\beta)$-modules which are finite dimensional over $\mathbf{k}$ is denoted by $R(\beta)$-gmod. In this paper, an $R(\beta)$-module means a graded $R(\beta)$-module, unless stated otherwise.

For a graded $R(\beta)$-module $M=\bigoplus_{k \in \mathbb{Z}} M_{k}$, we define $q M=\bigoplus_{k \in \mathbb{Z}}(q M)_{k}$, where $(q M)_{k}=M_{k-1}$. We call $q$ the grading shift functor on $\operatorname{Mod}_{\mathrm{gr}}(R(\beta))$. 
For $\beta, \gamma \in \mathrm{Q}^{+}$with $|\beta|=m$ and $|\gamma|=n$, we set the idempotent $e(\beta, \gamma)$ in $R(\beta+\gamma)$ :

$$
e(\beta, \gamma)=\sum_{\substack{\nu \in I^{\beta+\gamma} \\\left(\nu_{1}, \cdots, \nu_{m}\right) \in I^{\beta}}} e(\nu) .
$$

Then we have the $\mathbf{k}$-algebra homomorphism

$$
R(\beta) \otimes R(\gamma) \rightarrow e(\beta, \gamma) R(\beta+\gamma) e(\beta, \gamma)
$$

sending

$$
\begin{aligned}
& e(\mu) \otimes e(\nu) \mapsto e(\mu * \nu) \\
& \left(\mu \in I^{\beta}, \nu \in I^{\gamma}\right), \\
& x_{k} \otimes 1 \mapsto x_{k} e(\beta, \gamma), \quad 1 \otimes x_{s} \mapsto x_{m+s} e(\beta, \gamma) \\
& (1 \leq k \leq m, 1 \leq s \leq n) \text {, } \\
& \tau_{k} \otimes 1 \mapsto \tau_{k} e(\beta, \gamma) \text {, } \\
& 1 \otimes \tau_{s} \mapsto \tau_{m+s} e(\beta, \gamma) \\
& (1 \leq k<m, 1 \leq s<n) \text {, }
\end{aligned}
$$

where $\mu * \nu$ is the concatenation of $\mu$ and $\nu$; i.e., $\mu * \nu=\left(\mu_{1}, \ldots, \mu_{m}, \nu_{1}, \ldots, \nu_{n}\right)$.

For an $R(\beta)$-module $M$ and an $R(\gamma)$-module $N$, we define the convolution product $M \circ N$ by

$$
M \circ N:=R(\beta+\gamma) e(\beta, \gamma) \otimes_{R(\beta) \otimes R(\gamma)}(M \otimes N) .
$$

Then the category $R$-gmod $:=\bigoplus_{\beta \in Q^{+}} R(\beta)$-gmod becomes a tensor category in the sense of [KKK18, Appendix A.1] induced by the convolution product.

For an $R(\beta)$-module $M$ and an $R(\gamma)$-module $N$, we define the convolution product $M \circ N$ by

$$
M \circ N=R(\beta+\gamma) e(\beta, \gamma) \otimes_{R(\beta) \otimes R(\gamma)}(M \otimes N) .
$$

For $M \in R(\beta)$-gmod, the dual space

$$
M^{*}:=\operatorname{Hom}_{\mathbf{k}}(M, \mathbf{k})
$$

admits an $R(\beta)$-module structure via

$$
(r \cdot f)(u):=f(\psi(r) u) \quad(r \in R(\beta), u \in M),
$$

where $\psi$ denotes the $\mathbf{k}$-algebra anti-involution on $R(\beta)$ which fixes the generators $e(\nu), x_{m}$ and $\tau_{k}$ for $\nu \in I^{\beta}, 1 \leq m \leq|\beta|$ and $1 \leq k<|\beta|$.

A simple module $M$ in $R$-gmod is called self-dual if $M^{*} \simeq M$. Every simple module is isomorphic to a grading shift of a self-dual simple module ([KL09, §3.2]).

Theorem 5.2 ([KL09, Rou08]). For a symmetrizable Cartan datum $\left(\mathrm{A}, P, \Pi, P^{\vee}, \Pi^{\vee},(\cdot, \cdot)\right)$, let us denote by $U_{q}(\mathrm{~g})$ the corresponding quantum group and by $R$ the corresponding quiver Hecke algebra with $\left(Q_{i, j}\right)_{i, j \in I}$ in (5.1). Then there exists an $\mathbb{A}$-algebra isomorphism

$$
\Psi: U_{\mathbb{A}}^{-}(\mathrm{g})^{\vee} \simeq K(R \text {-gmod }),
$$

where $K(R$-gmod) denotes the Grothendieck ring of $R$-gmod and

$$
U_{\mathbb{A}}^{-}(\mathrm{g})^{\vee}:=\left\{x \in U_{q}^{-}(\mathrm{g}) \mid(x, y) \in \mathbb{A} \text { for all } y \in U_{\mathbb{A}}^{-}(\mathrm{g})\right\} .
$$

Definition 5.3. A quiver Hecke algebra $R(\beta)$ is symmetric if $Q_{i, j}(u, v)$ is a polynomial in $\mathbf{k}[u-v]$ for all $i, j \in \operatorname{supp}(\beta)$. In particular, we say that the quiver Hecke algebra $R$ is symmetric if $\mathrm{A}$ is symmetric and $\mathbb{Q}_{i j}(u, v)$ is a polynomial in $u-v$ for all $i, j \in I$.

Theorem 5.4 ([Rou12, VV11]). Assume that the quiver Hecke algebra $R$ is symmetric and $\mathbf{k}$ is of characteristic zero. Then under the isomorphism in Theorem 5.2, the dual canonical/upper global basis $\mathbf{B}^{\mathrm{up}}$ of $U_{\mathbb{A}}^{-}(\mathrm{g})^{\vee}$ corresponds to the set of the isomorphism classes of self-dual simple $R$-modules. 
5.2. $R$-matrices for quiver Hecke algebras. For $|\beta|=n$ and $1 \leq k<n$, define the intertwiner $\varphi_{k} \in R(\beta)$ by

$$
\varphi_{k} e(\nu)= \begin{cases}\left(\tau_{k} x_{k}-x_{k} \tau_{k}\right) e(\nu) & \text { if } \nu_{k}=\nu_{k+1}, \\ \tau_{k}(\nu) & \text { otherwise. }\end{cases}
$$

Since the intertwiners $\left\{\varphi_{k}\right\}_{1 \leq k \leq n-1}$ satisfies the braid relation, we can define the element $\varphi_{w}$ for each $w \in \mathfrak{S}_{n}$. For $m, n \in \mathbb{Z}_{\geq} 0$, we set $w[m, n] \in \mathfrak{S}_{m+n}$ such that

$$
w[m, n](k)= \begin{cases}k+n & \text { if } 1 \leq k \leq m, \\ k-m & \text { if } m<k \leq m+n .\end{cases}
$$

We define another symmetric bilinear form $(\cdot, \cdot)_{\mathrm{n}}$ on $\mathrm{Q}$ by $\left(\alpha_{i}, \alpha_{j}\right)_{\mathrm{n}}=\delta_{i j}$.

For an $R(\beta)$-module $M$ and an $R(\gamma)$-module with $\beta, \gamma \in \mathrm{Q}^{+}$such that $|\beta|=m,|\gamma|=n$, the map

$$
M \otimes N \rightarrow q^{(\beta, \gamma)-2(\beta, \gamma)_{\mathrm{n}}} N \circ M \quad \text { given by } u \otimes v \mapsto \varphi_{w[n, m]}(v \otimes u)
$$

is an $R(\beta) \otimes R(\gamma)$-homomorphism by [KKK18, Lemma 1.3.1] and it extends to an $R(\beta+\gamma)$ homomorphism

$$
R_{M, N}: M \circ N \rightarrow q^{(\beta, \gamma)-2(\beta, \gamma) \mathrm{n}} N \circ M .
$$

From now on, we assume that quiver Hecke algebras are symmetric. Let $z$ be an indeterminate that is homogeneous of degree 2 , and $\psi_{z}: R(\beta) \longrightarrow \mathbf{k}[z] \otimes R(\beta)$ be the algebra given by

$$
\psi_{z}\left(x_{k}\right)=x_{k}+z, \quad \psi_{z}\left(\tau_{k}\right)=\tau_{k}, \quad \psi_{z}(e(\nu))=e(\nu) .
$$

For an $R(\beta)$-module $M$, we denote by $M_{z}$ the $(\mathbf{k}[z] \otimes R(\beta))$-module $\mathbf{k}[z] \otimes M$ with the action of $R(\beta)$ twisted by $\psi_{z}$ :

$$
e(\nu)(a \otimes u)=a \otimes e(\nu) u, \quad x_{k}(a \otimes u)=(z a) \otimes u+a \otimes\left(x_{k} u\right), \quad \tau_{k}(a \otimes u)=a \otimes\left(\tau_{k} u\right),
$$

for $\nu \in I^{\beta}, a \in \mathbf{k}[u]$ and $u \in M$.

For a non-zero $R(\beta)$-module $M$ and a non-zero $R(\gamma)$-module $N$,

let $s$ be the order of zero of $R_{M_{z}, N_{z^{\prime}}}: M_{z} \circ N_{z^{\prime}} \rightarrow q^{(\beta, \gamma)-2(\beta, \gamma)_{\mathrm{n}}} N_{z^{\prime}} \circ M_{z}$, i.e., the largest non-negative integer such that the image of $R_{M_{z}, N_{z^{\prime}}}$ is contained in $\left(z^{\prime}-z\right)^{s} q^{(\beta, \gamma)-2(\beta, \gamma)_{\mathrm{n}}} N_{z^{\prime}} \circ M_{z}$.

For a non-zero $R(\beta)$-module $M$ and a non-zero $R(\gamma)$-module $N$, we set

$$
\Lambda(M, N):=-(\beta, \gamma)+2(\beta, \gamma)_{\mathrm{n}}-2 s, \quad R_{M_{z}, N_{z}^{\prime}}^{\mathrm{ren}}:=\left(z^{\prime}-z\right)^{-s} R_{M_{z} \cdot N_{z^{\prime}}}
$$

and define

$$
\mathbf{r}_{M, N}:=\left.\left(R_{M_{z}, N_{z}^{\prime}}^{\mathrm{ren}}\right)\right|_{z=z^{\prime}=0}: M \circ N \rightarrow q^{-\Lambda(M, N)} N \circ M .
$$

By [KKK18, Lemma 1.4.4 (ii)], $\mathbf{r}_{M, N}$ does not vanish.

We say that a simple $R(\beta)$-module $M$ is real if $M \circ M$ is simple.

Remark 5.5. We shall work always in the category of finite dimensional graded modules. For the sake of simplicity, we simply say that $M$ is an R-module instead of saying that $M$ is a finite dimensional graded $R(\beta)$-module for $\beta \in \mathrm{Q}^{+}$. We also sometimes ignore grading shifts if there is no danger of confusion. Hence, for $R$-modules $M$ and $N$, we sometimes say that $f: M \rightarrow N$ is a homomorphism if $f: q^{a} M \rightarrow N$ is a morphism in $R$-gmod for some $a \in \mathbb{Z}$. If we want to emphasize that $f: q^{a} M \rightarrow N$ is a morphism in $R$-gmod, we say so. 
Theorem 5.6 ([KKKO15a, Theorem 3.2]). Let $M$ be a simple $R(\alpha)$-module and $N$ be a simple $R(\beta)$-module, one of which is real. Then there exist unique non-zero homomorphisms (up to constant)

$$
\mathbf{r}_{M, N}: M \circ N \longrightarrow N \circ M \quad \text { and } \quad \mathbf{r}_{N, M}: N \circ M \longrightarrow M \circ N
$$

satisfying the following properties:

(i) $M \circ N$ has a simple socle and a simple head.

(ii) The socle and head of $M \circ N$ are distinct and appear once in the composition series of $M \circ N$ unless $M \circ N \simeq N \circ M$ is simple.

Proposition 5.7 ([KKKO15a, Corollary 3.11]). Let $M_{k}$ be a finite dimensional graded R-module $(k=1,2,3)$, and let $\varphi_{1}: L \rightarrow M_{1} \circ M_{2}$ and $\varphi_{2}: M_{2} \circ M_{3} \rightarrow L^{\prime}$ be non-zero homomorphisms. Assume further that $M_{2}$ is simple. Then the composition

$$
L \circ M_{3} \stackrel{\varphi_{1} \circ M_{3}}{\longrightarrow} M_{1} \circ M_{2} \circ M_{3} \stackrel{M_{1} \circ \varphi_{2}}{\longrightarrow} M_{1} \circ L^{\prime}
$$

does not vanish. Similarly, for non-zero homomorphisms $\varphi_{1}: L \rightarrow M_{2} \circ M_{3}, \varphi_{2}: M_{1} \circ M_{2} \rightarrow L^{\prime}$ and an assumption that $M_{2}$ is simple, we have a non-zero composition

$$
M_{1} \circ L \stackrel{M_{1} \circ \varphi_{1}}{\longrightarrow} M_{1} \circ M_{2} \circ M_{3} \stackrel{\varphi_{2} \circ M_{3}}{\longrightarrow} L^{\prime} \circ M_{3} .
$$

Theorem 5.8 ([BKM14, Kat14, KR11, McN15, Oh19]). For a finite simple Lie algebra g, we choose a reduced expression $\widetilde{w}_{0}$ of the longest element $w_{0}$. Let $R$ be the quiver Hecke algebra corresponding to $\mathrm{g}$. For each positive root $\beta \in \Phi^{+}$, there exists a self-dual simple module $S_{\left[\widetilde{w}_{0}\right]}(\beta)$ satisfying the following properties:

(a) $S_{\left[\widetilde{w}_{0}\right]}(\beta)^{\mathrm{O} m}$ is a real simple $R(m \beta)$-module.

(b) For every $\underline{m}_{\widetilde{w}_{0}} \in \mathbb{Z}_{\geq 0}^{\mathrm{N}}$, there exists a unique non-zero $R$-module homomorphism (up to constant)

$$
\vec{S}_{\left[\widetilde{w}_{0}\right]}(\underline{m}) \stackrel{\mathbf{r}_{\underline{m}}}{\rightarrow} \overleftarrow{S}_{\left[\widetilde{w}_{0}\right]}(\underline{m})
$$

where

$\vec{S}_{\left[\widetilde{w}_{0}\right]}(\underline{m}):=S_{\left[\widetilde{w}_{0}\right]}\left(\beta_{1}\right)^{\circ m_{1}} \circ \cdots \circ S_{\left[\widetilde{w}_{0}\right]}\left(\beta_{\mathrm{N}}\right)^{\circ m_{\mathrm{N}}}, \quad \overleftarrow{S}_{\left[\widetilde{w}_{0}\right]}(\underline{m}):=S_{\left[\widetilde{w}_{0}\right]}\left(\beta_{\mathrm{N}}\right)^{\circ m_{\mathrm{N}}} \circ \cdots \circ S_{\left[\widetilde{w}_{0}\right]}\left(\beta_{1}\right)^{\circ m_{1}}$

such that $\operatorname{Im}\left(\underline{\mathbf{r}}_{\underline{m}}\right) \simeq \operatorname{hd}\left(\vec{S}_{\left[\widetilde{w}_{0}\right]}(\underline{m})\right) \simeq \operatorname{soc}\left(\overleftarrow{S}_{\left[\widetilde{w}_{0}\right]}(\underline{m})\right)$ is simple.

(c) For any sequence $\underline{m}_{\widetilde{w}_{0}} \in \mathbb{Z}_{\geq 0}^{\ell\left(w_{0}\right)}$, we have

$$
\left[\vec{S}_{\left[\widetilde{w}_{0}\right]}(\underline{m})\right] \in\left[\operatorname{Im}\left(\mathbf{r}_{\underline{m}}\right)\right]+\sum_{\underline{m}^{\prime} \prec_{\left[\tilde{w}_{0}\right]}^{b} \underline{m}} \mathbb{Z}_{\geq 0}\left[\operatorname{Im}\left(\mathbf{r}_{\underline{m}^{\prime}}\right)\right] .
$$

(d) For distinct $\underline{m}, \underline{m}^{\prime} \in \mathbb{Z}_{\geq 0}^{\ell\left(w_{0}\right)}$, the simple modules $\operatorname{Im}\left(\mathbf{r}_{\underline{m}}\right)$ and $\operatorname{Im}\left(\mathbf{r}_{\underline{m}^{\prime}}\right)$ are distinct.

(e) For every simple $R$-module $M$, there exists a unique sequence $\underline{m} \in \mathbb{Z}_{\geq 0}^{\ell\left(w_{0}\right)}$ such that $M \simeq$ $\operatorname{Im}\left(\underline{\mathbf{r}}_{\underline{m}}\right) \simeq \operatorname{hd}\left(\vec{S}_{\left[\widetilde{w}_{0}\right]}(\underline{m})\right)$.

(f) For any $\left[\widetilde{w}_{0}\right]$-minimal pair $\left(\beta_{k}^{\widetilde{w}_{0}}, \beta_{l}^{\widetilde{w}_{0}}\right)$ of $\beta_{j}^{\widetilde{w}_{0}}=\beta_{k}^{\widetilde{w}_{0}}+\beta_{l}^{\widetilde{w}_{0}}$, there exists an exact sequence

$$
0 \rightarrow S_{\left[\widetilde{w}_{0}\right]}\left(\beta_{j}\right) \rightarrow S_{\left[\widetilde{w}_{0}\right]}\left(\beta_{k}\right) \circ S_{\left[\widetilde{w}_{0}\right]}\left(\beta_{l}\right) \stackrel{\mathbf{r}_{e_{k}+e_{l}}}{\longrightarrow} S_{\left[\widetilde{w}_{0}\right]}\left(\beta_{l}\right) \circ S_{\left[\widetilde{w}_{0}\right]}\left(\beta_{k}\right) \rightarrow S_{\left[\widetilde{w}_{0}\right]}\left(\beta_{j}\right) \rightarrow 0 .
$$


Moreover, under the isomorphism $\Psi$ in $(5.2)$, each isomorphism class $\left[S_{\left[\widetilde{w}_{0}\right]}(\beta)\right]$ of $S_{\left[\widetilde{w}_{0}\right]}(\beta)\left(\beta \in \Phi^{+}\right)$ is mapped onto the dual root vector $\mathbf{F}_{\left[\widetilde{w}_{0}\right]}^{\mathrm{up}}(\beta)$ of the dual $P B W$ basis $P_{\left[\widetilde{w}_{0}\right]}$ of $U_{\mathbb{A}}^{-}\left(\mathfrak{g}_{0}\right)^{\vee}$ (up to $q^{\mathbb{Z}}$ ), which is associated to the commutation class $\left[\widetilde{w}_{0}\right]$ of $w_{0}$ (see [KKK15, §4] for more details).

5.3. Generalized Schur-Weyl duality functors. In this subsection, we recall the generalized Schur-Weyl duality functors constructed in [KKK18].

Let $U_{q}^{\prime}(\mathfrak{g})$ be a quantum affine algebra over $\mathbf{k}$. Assume that we are given an index set $J$, a family of good modules $\left\{V_{j}\right\}_{j \in J}$ and a map $X: J \rightarrow \mathbf{k}^{\times}$. We associate a quiver $\Gamma^{J}$ from the datum $\left(J, X,\left\{V_{j}\right\}_{j \in J}\right)$ as follows:

(1) Take $J$ as the set of vertices.

(2) Put $d_{i j}$-many arrows from $i$ to $j$, where $d_{i j}$ denotes the order of zero of $d_{V_{i}, V_{j}}\left(z_{j} / z_{i}\right)$ at $z_{j} / z_{i}=$ $X(j) / X(i)$.

Note that $d_{i j} d_{j i}=0$ for $i, j \in J$.

We define a symmetric Cartan matrix $\mathrm{A}^{J}=\left(a_{i j}^{J}\right)$ and a family of polynomials $\left(P_{i j}^{J}\right)_{i, j \in J}$ by

$$
a_{i j}^{J}=\left\{\begin{array}{cl}
2 & \text { if } i=j, \\
-d_{i j}-d_{j i} & \text { if } i \neq j,
\end{array} \quad \text { and } \quad P_{i, j}^{J}(u, v)=(u-v)^{d_{i j}}\right.
$$

We denote by $\mathrm{Q}_{J}^{+}$the root lattice and $\left\{\alpha_{i}^{J}\right\}_{i \in J}$ the set of simple roots associated to $\mathrm{A}^{J}$.

Let us denote by $R^{J}(\beta)$ the symmetric quiver Hecke algebra associated with the Cartan matrix $\mathrm{A}^{J}$ and a matrix $\left(Q_{i, j}^{J}\right)_{i, j}$ in $(5.1)$, where

$$
Q_{i, j}^{J}=\left(1-\delta_{i, j}\right) P_{i, j}^{J}(u, v) P_{j, i}^{J}(v, u) .
$$

For each $i \in J$, let $L(i)$ be the 1-dimensional $R^{J}\left(\alpha_{i}\right)$-module generated by a nonzero vector $u(i)$ with relation $x_{1} u(i)=0$ and $e(j) u(i)=\delta_{j, i} u(i)$ for $j \in J$. The space $L(i)_{z}:=\mathbf{k}[z] \otimes L(i)$ admits an $R^{J}\left(\alpha_{i}\right)$-module structure as follows:

$$
x_{1}(a \otimes u(i))=(z a) \otimes u(i), \quad e(j)(a \otimes u(i))=\delta_{j, i}(a \otimes u(i)) .
$$

Theorem 5.9. [KKK18, Theorem 3.3] For each $\beta \in \mathrm{Q}_{J}^{+}$, there exists a $\left(U_{q}^{\prime}(\mathfrak{g}), R^{J}(\beta)\right)$-bimodule $\widehat{V}^{\otimes \beta}$ which induces the functor

$$
\mathcal{F}_{\beta}: \operatorname{Mod}\left(R^{J}(\beta)\right) \rightarrow \operatorname{Mod}\left(U_{q}^{\prime}(\mathfrak{g})\right) \quad \text { given by } \quad \mathcal{F}_{\beta}(M):=\widehat{V}^{\otimes \beta} \otimes_{R^{J}(\beta)} M .
$$

In particular, for any $i \in J$, we have

$$
\mathcal{F}(L(i)) \simeq\left(V_{i}\right)_{X(i)} \quad \text { for } i \in J
$$

Theorem 5.10 ([KKK18]). When we restrict the functor $\mathcal{F}$ to $R^{J}$-gmod, we have

$$
\mathcal{F}: \bigoplus_{\beta \in Q_{J}^{+}} R^{J}(\beta)-\operatorname{gmod} \rightarrow \mathcal{C}_{\mathfrak{g}} .
$$

Moreover, $\mathcal{F}$ satisfies the following properties:

(a) $\mathcal{F}$ is a tensor functor; that is, for any $M_{1}, M_{2} \in R^{J}$-gmod, we have

$$
\mathcal{F}\left(R^{J}(0)\right) \simeq \mathbf{k} \quad \text { and } \quad \mathcal{F}\left(M_{1} \circ M_{2}\right) \simeq \mathcal{F}\left(M_{1}\right) \otimes \mathcal{F}\left(M_{2}\right) .
$$

(b) If the quiver $\Gamma^{J}$ is a Dynkin quiver of finite type $A_{n}, D_{n}$ or $E_{6,7,8}$, then $\mathcal{F}$ is exact.

We call the functor $\mathcal{F}$ the generalized Schur-Weyl duality functor. 
Theorem 5.11 ([KKK15, KKKO16]). Let $U_{q}^{\prime}\left(\mathfrak{g}^{(t)}\right)$ be a quantum affine algebra of type $A_{n}^{(t)}$ (resp. $\left.D_{n}^{(t)}\right)$ and let $Q$ be a Dynkin quiver of finite type $A_{n}$ (resp. $\left.D_{n}\right)$ for $t=1,2$. Take $J$ and $\mathcal{S}$ as the set of simple roots $\Pi$ associated to $Q$. We define two maps

$$
s: \Pi \rightarrow\left\{V\left(\varpi_{i}\right) \mid i \in I_{0}\right\} \quad \text { and } \quad X: \Pi \rightarrow \mathbf{k}^{\times}
$$

as follows: for $\alpha \in \Pi$ with $\widehat{\Omega}_{Q}(\alpha)=(i, p)$, we define

$$
s(\alpha)=\left\{\begin{array}{ll}
V\left(\varpi_{i}\right) \quad \text { if } \mathfrak{g}^{(1)}=A_{n}^{(1)} \text { or } D_{n}^{(1)}, \\
V\left(\varpi_{i^{\star}}\right) & \text { if } \mathfrak{g}^{(2)}=A_{n}^{(2)} \text { or } D_{n}^{(2)},
\end{array} \quad X(\alpha)= \begin{cases}(-q)^{p} & \text { if } \mathfrak{g}^{(1)}=A_{n}^{(1)} \text { or } D_{n}^{(1)}, \\
\left((-q)^{p}\right)^{\star} & \text { if } \mathfrak{g}^{(2)}=A_{n}^{(2)} \text { or } D_{n}^{(2)} .\end{cases}\right.
$$

Then we have the following:

(1) The underlying graph of $\Gamma^{J}$ coincides with the one of $Q$. Hence the functor

$$
\mathcal{F}_{Q}^{(t)}: R^{J} \text {-gmod } \rightarrow \mathcal{C}_{Q}^{(t)} \quad(t=1,2) \text { in Theorem } 5.10 \text { is exact. }
$$

(2) The functor $\mathcal{F}_{Q}^{(t)}$ induces a bijection from the set of the isomorphism classes of simple objects of $R^{J}$-gmod to that of $\mathcal{C}_{Q}^{(t)}$. In particular, $\mathcal{F}_{Q}^{(t)}$ sends $S_{Q}(\beta):=S_{[Q]}(\beta)$ to $V_{Q}^{(t)}(\beta)$.

(3) The functors $\mathcal{F}_{Q}^{(1)}$ and $\mathcal{F}_{Q}^{(2)}$ induce the ring isomorphisms in Theorem 3.14. Moreover, the induced functor between $\mathcal{C}_{Q}^{(1)}$ and $\mathcal{C}_{Q}^{(2)}$ :

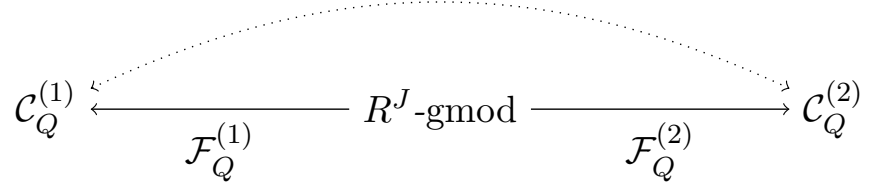

preserves dimensions and sends simples to simples, bijectively.

Remark 5.12. It was pointed out in [KKK15] that when $\mathfrak{g}^{(1)}$ is of type $E_{6,7,8}^{(1)}, Q$ is a Dynkin quiver of finite type $E_{6,7,8}$ and we choose $(J, \mathcal{S}, s, X)$ as in Theorem 5.11 , one can prove that (i) $\mathcal{F}_{Q}^{(1)}$ is exact (ii) $\mathcal{F}_{Q}^{(1)}$ sends $S_{Q}(\beta)$ to $V_{Q}(\beta)\left(\beta \in \Phi^{+}\right)$and (iii) $\mathcal{F}_{Q}^{(1)}$ induces a bijection from the set of the isomorphism classes of simple objects of $R^{J}$-gmod to that of $\mathcal{C}_{Q}^{(1)}$ provided that $\Gamma^{J}$ is of finite type $E_{6,7,8}$, by applying the same arguments in [KKK15, §4] (see also [KKK15, Conjecture 4.3.2]).

Theorem 5.13 ([KO18]). Let $U_{q}^{\prime}\left(\mathfrak{g}^{(1)}\right)$ be a quantum affine algebra of type $B_{n}^{(1)}$ (resp. $\left.C_{n}^{(1)}\right)$ and let $[Q 2]$ be a twisted adapted class of finite type $A_{2 n-1}\left(\right.$ resp. $\left.D_{n+1}\right)$. Take $J$ and $\mathcal{S}$ as the set of simple roots $\Pi$ of finite type $A_{2 n-1}$ (resp. $\left.D_{n+1}\right)$. We define two maps

$$
s: \Pi \rightarrow\left\{V\left(\varpi_{i}\right) \mid i \in I_{0}\right\} \quad \text { and } \quad X: \Pi \rightarrow \mathbf{k}^{\times}
$$

as follows: for $\alpha \in \Pi$ with $\widehat{\Omega}_{Q}(\alpha)=(i, p / \mathrm{d})$, we define

$$
s(\alpha)=V\left(\varpi_{i}\right), \quad X(\alpha)= \begin{cases}(-1)^{i}\left(q^{1 / \mathrm{d}}\right)^{p} & \text { if } \mathfrak{g}^{(1)}=B_{n}^{(1)}, \\ \left(-q^{1 / \mathrm{d}}\right)^{p} & \text { if } \mathfrak{g}^{(1)}=C_{n}^{(1)} .\end{cases}
$$

Then we have the following: 
(1) The underlying graph of $\Gamma^{J}$ is a Dynkin diagram of finite type $A_{2 n-1}\left(\right.$ resp. $\left.D_{n+1}\right)$. Hence the functor

$$
\mathscr{F}_{\mathscr{Q}}: R^{J} \operatorname{gmod} \rightarrow \mathscr{C}_{\mathscr{Q}}^{(1)} \text { in Theorem } 5.10 \text { is exact. }
$$

(2) The functor $\mathscr{F}_{\mathscr{Q}}^{(1)}$ induces a bijection from the set of the isomorphism classes of simple objects of $R^{J}$-gmod to that of $\mathscr{C}_{\mathscr{Q}}^{(1)}$. In particular, $\mathscr{F}_{\mathscr{Q}}$ sends $S_{\mathscr{Q}}(\beta):=S_{[\mathscr{Q}]}(\beta)$ to $V_{\mathscr{Q}}(\beta)$.

(3) The functors $\mathscr{F}_{\mathscr{Q}}$ induces the ring isomorphism in Theorem 3.17. Moreover, the induced functors $\operatorname{among} \mathcal{C}_{Q}^{(1)}, \mathcal{C}_{Q^{\prime}}^{(2)}$ and $\mathscr{C}_{\mathscr{Q}}^{(1)}$

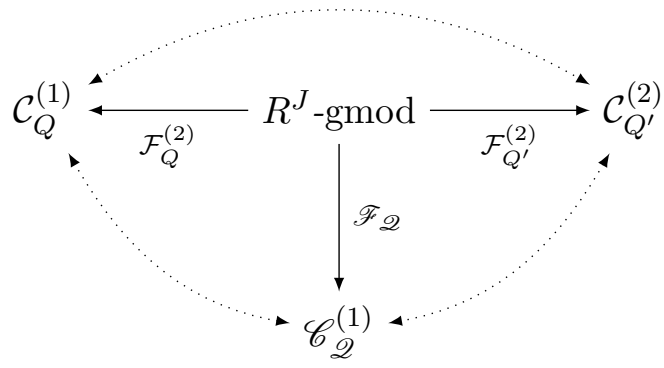

send simples to simples, bijectively.

\section{Application from generalized Schur-Weyl Duality}

In this section, we shall show the applications of the denominator formulas that can be obtained from the generalized Schur-Weyl duality functors.

6.1. $U_{q}^{\prime}\left(E_{6,7,8}^{(1)}\right)$. In this subsection, $Q$ always denotes a Dynkin quiver of finite type $E_{6,7,8}$ and $U_{q}^{\prime}(\mathfrak{g})$ denotes the quantum affine algebra of type $E_{6,7,8}^{(1)}$.

The following proposition can be checked with the denominator formulas given in the previous section.

Proposition 6.1. For the Dynkin quiver $Q$ given in Example 2.19 and Appendix A, B, take $J$ and $\mathcal{S}$ as the set of simple roots $\Pi$ associated to $Q$. We define two maps

$$
s: \Pi \rightarrow\left\{V\left(\varpi_{i}\right) \mid i \in I_{0}\right\} \quad \text { and } \quad X: \Pi \rightarrow \mathbf{k}^{\times}
$$

as follows: for $\alpha \in \Pi$ with $\widehat{\Omega}_{Q}(\alpha)=(i, p)$, we define

$$
s(\alpha)=V\left(\varpi_{i}\right), \quad X(\alpha)=(-q)^{p} .
$$

Then the underlying graph of $\Gamma^{J}$ coincides with the one of $Q$.

Proof. Note that the pairs of simple roots $\left(\alpha_{i}, \alpha_{j}\right)$ are [Q]-minimal if $i, j$ are adjacent in $\Delta$ and $[Q]$ simple, otherwise. Thus the underlying graph of $\Gamma^{J}$ coincides with the one of $Q$ by Theorem 4.31. 
Example 6.2. Let us see the $E_{6}$ case with an example. By forgetting the arrows of $\Gamma_{Q}$ in (2.6), the underlying graph of $\Gamma^{J}$ can be described as follows:

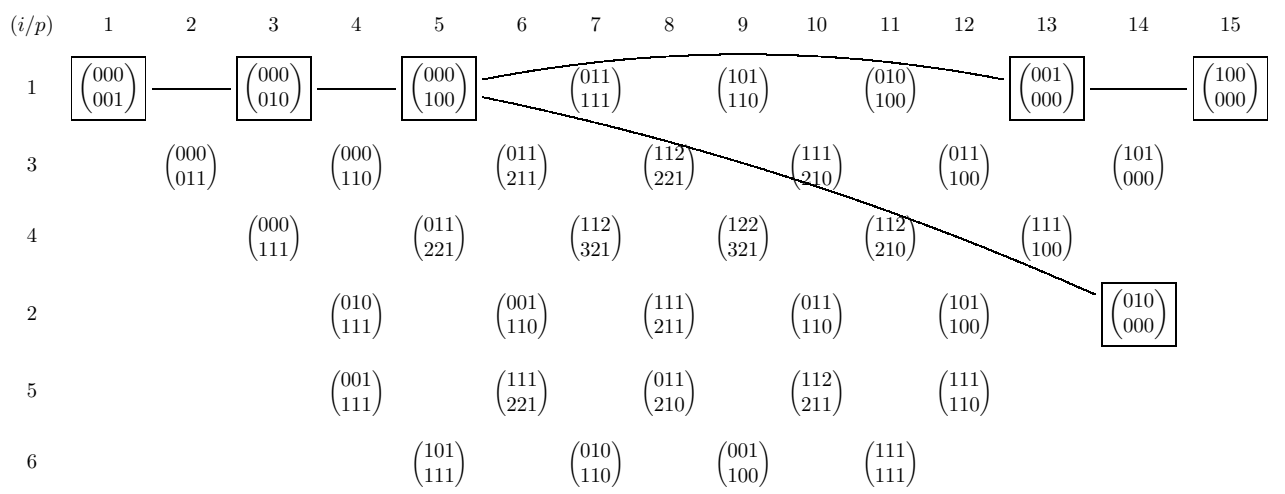

since

$$
d_{1,1}(z)=\left(z-q^{2}\right)\left(z-q^{8}\right) \quad \text { and } \quad d_{1,2}(z)=\left(z+q^{5}\right)\left(z+q^{9}\right) .
$$

Theorem 6.3. The above proposition holds for any $Q$ of type $E_{6,7,8}$. Hence for any $Q$ of type $E_{6,7,8}$, there exists an exact functor

$$
\mathcal{F}_{Q}^{(1)}: R^{E_{6,7,8} \operatorname{gmod} \rightarrow \mathcal{C}_{Q}^{(1)}}
$$

which sends $S_{Q}(\beta)$ to $V_{Q}(\beta)\left(\beta \in \Phi^{+}\right)$and $\mathcal{F}_{Q}^{(1)}$ induces a bijection from the set of the isomorphism classes of simple objects of $R^{E_{6,7,8}}-\operatorname{gmod}$ to that of $\mathcal{C}_{Q}^{(1)}$.

Proof. Our assertion follows from Remark 5.12.

6.2. $U_{q}^{\prime}\left(D_{4}^{(3)}\right)$ and $U_{q}^{\prime}\left(E_{6}^{(2)}\right)$. In this subsection, we shall prove the $U_{q}^{\prime}\left(D_{4}^{(3)}\right)$ and $U_{q}^{\prime}\left(E_{6}^{(2)}\right)$-analogues of Theorem 3.13, Theorem 3.14 and Theorem 5.11. Since the frameworks for $U_{q}^{\prime}\left(D_{4}^{(3)}\right)$ and $U_{q}^{\prime}\left(E_{6}^{(2)}\right)$ are the same, we shall give proofs for $U_{q}^{\prime}\left(D_{4}^{(3)}\right)$-case only.

Lemma 6.4. For Dynkin quivers $Q$ and $Q^{\prime}$ of the same finite type $A_{n}, D_{n}$ or $E_{6,7,8}$, assume that there exists a pair $(\alpha, \beta)$ of $\gamma$ contained in $\Phi_{[Q]}(i, j)(t)$, then there exists a pair $\left(\alpha^{\prime}, \beta^{\prime}\right)$ such that $\alpha^{\prime}+\beta^{\prime} \in \Phi^{+}$and $\left(\alpha^{\prime}, \beta^{\prime}\right)$ is contained in $\Phi_{\left[Q^{\prime}\right]}(i, j)(t)$ or $\Phi_{\left[Q^{\prime}\right]}\left(i^{*}, j^{*}\right)(t)$.

Proof. The assertion follows from well-definedness of the distance polynomial $D_{i, j}^{\llbracket \Delta \rrbracket}(z)$ and the fact that $\Gamma_{Q^{\prime}}$ can be obtained from $\Gamma_{Q}$ by applying the reflection functors properly.

Proposition 6.5. For any Dynkin quiver $Q$ of finite type $D_{4}(t=3)\left(\right.$ resp. $\left.E_{6}(t=2)\right)$, take $J$ and $\mathcal{S}$ as the set of simple roots $\Pi$ associated to $Q$. We define two maps

$$
s: \Pi \rightarrow\left\{V^{(t)}\left(\varpi_{i}\right) \mid i \in I_{0}\right\} \quad \text { and } \quad X: \Pi \rightarrow \mathbf{k}^{\times}
$$

as follows: for $\alpha \in \Pi$ with $\widehat{\Omega}_{Q}(\alpha)=(i, p)$, we define

$$
s(\alpha)=\left\{\begin{array}{ll}
V^{(3)}\left(\varpi_{i^{\dagger}}\right) & \text { if } Q \text { is of type } D_{4}, \\
V^{(2)}\left(\varpi_{i^{\star}}\right) & \text { if } Q \text { is of type } E_{6}
\end{array} \quad X(\alpha)= \begin{cases}\left((-q)^{p}\right)^{\dagger} & \text { if } Q \text { is of type } D_{4}, \\
\left((-q)^{p}\right)^{\star} & \text { if } Q \text { is of type } E_{6},\end{cases}\right.
$$


where $i^{\dagger}$ and $\left((-q)^{p}\right)^{\dagger}$ are defined in (3.9b) (resp. $i^{\star}$ and $\left((-q)^{p}\right)^{\star}$ are defined in $\left.(3.9 \mathrm{a})\right)$. Then the underlying graph of $\Gamma^{J}$ coincides with the Dynkin diagram of finite type $D_{4}$ (resp. $\left.E_{6}\right)$. Hence we have an exact functor

$$
\mathcal{F}_{Q}^{(3)}: R^{D_{4}} \operatorname{gmod} \rightarrow \mathcal{C}_{Q}^{(3)} \quad\left(\text { resp. } \mathcal{F}_{Q}^{(2)}: R^{E_{6}} \operatorname{gmod} \rightarrow \mathcal{C}_{Q}^{(2)}\right) .
$$

Proof. Our assertion follows from Theorem 5.11 on $U_{q}^{\prime}\left(D_{4}^{(1)}\right)$ and Corollary 4.11.

Example 6.6. By forgetting the arrows of $\Gamma_{Q}$ in (2.6), the underlying graph of $\Gamma^{J}$ can be described as follows:

\begin{tabular}{|c|c|c|c|c|c|c|c|}
\hline$(i, p)$ & 1 & 2 & 3 & 4 & 5 & 6 & 7 \\
\hline 1 & $\langle 1,-2\rangle$ & & $\langle 2,4\rangle$ & & $\langle 1,-4\rangle$ & & \\
\hline 2 & & $\langle 1,4\rangle$ & 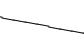 & $\langle 1,2\rangle$ & & $\langle 2,-4\rangle$ & \\
\hline 3 & & & $\langle 1,3\rangle$ & & $\langle 2,-3\rangle$ & 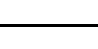 & $\langle 3,-4\rangle$ \\
\hline 4 & $\langle 3,4\rangle$ & & $\langle 1,-3\rangle$ & & $\langle 2,3\rangle$ & & \\
\hline
\end{tabular}

Now we shall prove the following theorem:

Theorem 6.7. For any Dynkin quiver $Q$ of finite type $D_{4}(t=3)$ or $E_{6}(t=2)$, and $\gamma \in \Phi^{+}$, we have

$$
\mathcal{F}_{Q}^{(t)}\left(S_{Q}(\gamma)\right) \simeq V_{Q}^{(t)}(\gamma)
$$

Proof. We shall prove the claim for the AR quiver $\Gamma_{Q}$ in (2.8) as the rest of the cases are similar. By Theorem 5.9, we have $\mathcal{F}_{Q}^{(3)}\left(S_{Q}\left(\alpha_{i}\right)\right) \simeq V_{Q}^{(3)}\left(\alpha_{i}\right)$. Hence,

$$
\begin{array}{lll}
\mathcal{F}_{Q}^{(3)}\left(S\left(\alpha_{1}\right)\right) \simeq V^{(3)}\left(\varpi_{1}\right)_{-q}, & \mathcal{F}_{Q}^{(3)}\left(S\left(\alpha_{2}\right)\right) \simeq V^{(3)}\left(\varpi_{1}\right)_{-\omega q^{5}}, \\
\mathcal{F}_{Q}^{(3)}\left(S\left(\alpha_{3}\right)\right) \simeq V^{(3)}\left(\varpi_{1}\right)_{-\omega q^{7}}, & & \mathcal{F}_{Q}^{(3)}\left(S\left(\alpha_{4}\right)\right) \simeq V^{(3)}\left(\varpi_{1}\right)_{-\omega^{2} q} .
\end{array}
$$

Thus, we have an exact sequence

$$
0 \rightarrow S_{Q}\left(\alpha_{2}+\alpha_{3}\right) \rightarrow S_{Q}\left(\alpha_{3}\right) \circ S_{Q}\left(\alpha_{2}\right) \stackrel{\text { r }}{\rightarrow} S_{Q}\left(\alpha_{2}\right) \circ S_{Q}\left(\alpha_{3}\right) \rightarrow S_{Q}\left(\alpha_{2}+\alpha_{3}\right) \rightarrow 0 .
$$

Applying the exact functor $\mathcal{F}_{Q}^{(3)}$, we have

$$
\begin{aligned}
0 \rightarrow \mathcal{F}_{Q}^{(3)}\left(S_{Q}\left(\alpha_{2}+\alpha_{3}\right)\right) & \rightarrow V^{(3)}\left(\varpi_{1}\right)_{-\omega q^{7}} \otimes V^{(3)}\left(\varpi_{1}\right)_{-\omega q^{5}} \\
& \stackrel{\mathcal{F}_{Q}^{(3)}(\mathbf{r})}{\longrightarrow} V^{(3)}\left(\varpi_{1}\right)_{-\omega q^{5}} \otimes V^{(3)}\left(\varpi_{1}\right)_{-\omega q^{7}} \rightarrow \mathcal{F}_{Q}^{(3)}\left(S_{Q}\left(\alpha_{2}+\alpha_{3}\right)\right) \rightarrow 0 .
\end{aligned}
$$

By the Dorey's type homomorphism and the argument of [KO18, Theorem 7.3], we have

$$
\mathcal{F}_{Q}^{(3)}\left(S_{Q}\left(\alpha_{2}+\alpha_{3}\right)\right) \simeq V^{(3)}\left(\varpi_{2}\right)_{-q^{6}} \simeq V^{(3)}\left(\varpi_{2}\right)_{-\omega^{t} q^{6}} \quad(t=1,2) .
$$

Applying the same argument to the minimal pairs $\left(\alpha_{1}, \alpha_{2}+\alpha_{3}\right)$ and $\left(\alpha_{4}, \alpha_{2}+\alpha_{3}\right)$, we have

$$
\mathcal{F}_{Q}^{(3)}\left(S_{Q}(\langle 1,-4\rangle)\right) \simeq V^{(3)}\left(\varpi_{1}\right)_{-q^{5}}, \quad \mathcal{F}_{Q}^{(3)}\left(S_{Q}(\langle 2,3\rangle)\right) \simeq V^{(3)}\left(\varpi_{1}\right)_{-w^{2} q^{5}} .
$$

By [Her10, page 39; arxiv version], we have the six-term exact sequence

$$
\begin{aligned}
0 & \rightarrow V^{(3)}\left(\varpi_{1}\right)_{-q^{5}} \otimes V^{(3)}\left(\varpi_{1}\right)_{-w q^{5}} \otimes V^{(3)}\left(\varpi_{1}\right)_{-w^{2} q^{5}} \rightarrow V^{(3)}\left(\varpi_{2}\right)_{-q^{6}} \otimes V^{(3)}\left(\varpi_{2}\right)_{-q^{4}} \\
& \rightarrow V^{(3)}\left(\varpi_{2}\right)_{-q^{4}} \otimes V^{(3)}\left(\varpi_{2}\right)_{-q^{6}} \rightarrow V^{(3)}\left(\varpi_{1}\right)_{-q^{5}} \otimes V^{(3)}\left(\varpi_{1}\right)_{-w q^{5}} \otimes V^{(3)}\left(\varpi_{1}\right)_{-w^{2} q^{5}} \rightarrow 0
\end{aligned}
$$


Note that we have an exact sequence

$$
\begin{aligned}
0 & \rightarrow S_{Q}(\langle 1,-4\rangle) \circ S_{Q}(\langle 2,-3\rangle) \circ S_{Q}(\langle 2,3\rangle) \rightarrow S_{Q}(\langle 2,-4\rangle) \circ S_{Q}(\langle 1,2\rangle) \\
& \rightarrow S_{Q}(\langle 1,2\rangle) \circ S_{Q}(\langle 2,-4\rangle) \rightarrow S_{Q}(\langle 1,-4\rangle) \circ S_{Q}(\langle 2,-3\rangle) \circ S_{Q}(\langle 2,3\rangle) \rightarrow 0 .
\end{aligned}
$$

Furthermore, $V^{(3)}\left(\varpi_{1}\right)_{-q^{5}} \otimes V^{(3)}\left(\varpi_{1}\right)_{-w q^{5}} \otimes V^{(3)}\left(\varpi_{1}\right)_{-w^{2} q^{5}}$ is simple. By applying the exact functor $\mathcal{F}_{Q}^{(3)}$ to $(6.5)$, we have

$$
\begin{aligned}
0 & \rightarrow V^{(3)}\left(\varpi_{1}\right)_{-q^{5}} \otimes V^{(3)}\left(\varpi_{1}\right)_{-w q^{5}} \otimes V^{(3)}\left(\varpi_{1}\right)_{-w^{2} q^{5}} \rightarrow V^{(3)}\left(\varpi_{2}\right)_{-q^{6}} \otimes \mathcal{F}_{Q}^{(3)}\left(S_{Q}(\langle 1,2\rangle)\right) \\
& \rightarrow \mathcal{F}_{Q}^{(3)}\left(S_{Q}(\langle 1,2\rangle)\right) \otimes V^{(3)}\left(\varpi_{2}\right)_{-q^{6}} \rightarrow V^{(3)}\left(\varpi_{1}\right)_{-q^{5}} \otimes V^{(3)}\left(\varpi_{1}\right)_{-w q^{5}} \otimes V^{(3)}\left(\varpi_{1}\right)_{-w^{2} q^{5}} \rightarrow 0 .
\end{aligned}
$$

In particular, we have

$$
\mathcal{F}_{Q}^{(3)}\left(S_{Q}(\langle 1,2\rangle)\right) \otimes V^{(3)}\left(\varpi_{2}\right)_{-q^{6}} \rightarrow V^{(3)}\left(\varpi_{1}\right)_{-q^{5}} \otimes V^{(3)}\left(\varpi_{1}\right)_{-w q^{5}} \otimes V^{(3)}\left(\varpi_{1}\right)_{-w^{2} q^{5}} .
$$

Since every module in $\mathcal{C}_{\mathfrak{g}}$ has a dual, by taking the dual $V^{(3)}\left(\varpi_{2}\right)_{-q^{0}}$ of $V^{(3)}\left(\varpi_{2}\right)_{-q^{6}}$, we have

$$
\mathcal{F}_{Q}^{(3)}\left(S_{Q}(\langle 1,2\rangle)\right) \simeq V^{(3)}\left(\varpi_{2}\right)_{-q^{4}}
$$

with Theorem 5.6.

By [Her10, page 39; arxiv version], we have the six-term exact sequence

$0 \rightarrow V^{(3)}\left(\varpi_{2}\right)_{-q^{4}} \rightarrow V^{(3)}\left(\varpi_{1}\right)_{-q^{5}} \otimes V^{(3)}\left(\varpi_{1}\right)_{-q^{3}} \rightarrow V^{(3)}\left(\varpi_{1}\right)_{-q^{3}} \otimes V^{(3)}\left(\varpi_{1}\right)_{-q^{5}} \rightarrow V^{(3)}\left(\varpi_{2}\right)_{-q^{4}} \rightarrow 0$.

Note that we have an exact sequence

$$
0 \rightarrow S_{Q}(\langle 1,2\rangle) \rightarrow S_{Q}(\langle 1,-4\rangle) \circ S_{Q}(\langle 2,3\rangle) \rightarrow S_{Q}(\langle 2,3\rangle) \circ S_{Q}(\langle 1,-4\rangle) \rightarrow S_{Q}(\langle 1,2\rangle) \rightarrow 0 .
$$

By applying the exact functor $\mathcal{F}_{Q}^{(3)}$ to $(6.6)$, we have

$$
\begin{aligned}
0 \rightarrow V^{(3)}\left(\varpi_{2}\right)_{-q^{4}} & \rightarrow V^{(3)}\left(\varpi_{1}\right)_{-q^{5}} \otimes \mathcal{F}_{Q}^{(3)}\left(S_{Q}(\langle 2,4\rangle)\right) \\
& \rightarrow \mathcal{F}_{Q}^{(3)}\left(S_{Q}(\langle 2,4\rangle)\right) \otimes V^{(3)}\left(\varpi_{1}\right)_{-q^{5}} \rightarrow V^{(3)}\left(\varpi_{2}\right)_{-q^{4}} \rightarrow 0
\end{aligned}
$$

In particular, we have

$$
\mathcal{F}_{Q}^{(3)}\left(S_{Q}(\langle 2,4\rangle)\right) \otimes V^{(3)}\left(\varpi_{1}\right)_{-q^{5}} \rightarrow V^{(3)}\left(\varpi_{2}\right)_{-q^{4}}
$$

By taking the dual $V^{(3)}\left(\varpi_{1}\right)_{-q^{-1}}$ of $V^{(3)}\left(\varpi_{1}\right)_{-q^{5}}$, we have $\mathcal{F}_{Q}^{(3)}\left(S_{Q}(\langle 2,4\rangle)\right) \simeq V^{(3)}\left(\varpi_{1}\right)_{-q^{3}}$.

Since $\alpha_{2}$ and $\alpha_{4}$ is a pair for $\alpha_{2}+\alpha_{4}=\langle 2,4\rangle$, the fact that $\mathcal{F}_{Q}^{(3)}\left(S_{Q}(\langle 2,4\rangle)\right) \simeq V^{(3)}\left(\varpi_{1}\right)_{-q^{3}}$ implies

$$
V^{(3)}\left(\varpi_{1}\right)_{-w^{2} q^{1}} \otimes V^{(3)}\left(\varpi_{1}\right)_{-w q^{5}} \rightarrow V^{(3)}\left(\varpi_{1}\right)_{-q^{3}} .
$$

Using (6.7), we can obtain,

$$
\mathcal{F}_{Q}^{(3)}\left(S_{Q}(\langle 1,-3\rangle)\right) \simeq V^{(3)}\left(\varpi_{1}\right)_{-w^{2} q^{3}}, \quad \mathcal{F}_{Q}^{(3)}\left(S_{Q}(\langle 1,3\rangle)\right) \simeq V^{(3)}\left(\varpi_{1}\right)_{-w q^{3}} .
$$

Finally, we can obtain $\mathcal{F}_{Q}^{(3)}\left(S_{Q}(\langle 1,4\rangle)\right) \simeq V^{(3)}\left(\varpi_{2}\right)_{-q^{2}}$ by using (6.4).

Now we have shown that for the Dynkin quiver $Q$

(a) $\mathcal{F}_{Q}^{(3)}\left(S_{Q}(\beta)\right) \simeq V_{Q}^{(3)}(\beta)$ for all $\beta \in \Phi^{+}$and

(b) $V_{Q}^{(3)}(\beta) \otimes V_{Q}^{(3)}(\alpha) \rightarrow V_{Q}^{(3)}(\gamma)$ for every $[Q]$-minimal pair $(\alpha, \beta)$ of $\gamma \in \Phi^{+}$. 
Furthermore, for any pair of $(\alpha, \beta)$ of $\gamma \in \Phi^{+}$, it is proved in [Oh19, Theorem 5.20] that there exists a six-term exact sequence

$$
0 \rightarrow S_{Q}(\gamma) \rightarrow S_{Q}(\alpha) \circ S_{Q}(\beta) \rightarrow S_{Q}(\beta) \circ S_{Q}(\alpha) \rightarrow S_{Q}(\gamma) \rightarrow 0 .
$$

Hence we can prove that

$$
V_{Q}^{(3)}(\beta) \otimes V_{Q}^{(3)}(\alpha) \rightarrow V_{Q}^{(3)}(\gamma)
$$

for any pair $(\alpha, \beta)$ of $\gamma \in \Phi^{+}$, by applying the same argument.

We have shown that our assertion holds for the $Q$. By Lemma 6.4, we can apply the induction argument on $|\gamma|$ for any $Q^{\prime}$ of finite type $D_{4}$.

Corollary 6.8. For $U_{q}^{\prime}\left(D_{4}^{(3)}\right)(t=3)$ or $U_{q}^{\prime}\left(E_{6}^{(2)}\right)(t=2)$, let $(i, x),(j, y),(k, z) \in I_{0} \times \mathbf{k}^{\times}$. Then

$$
\operatorname{Hom}_{U_{q}^{\prime}\left(\mathfrak{g}^{(t)}\right)}\left(V^{(t)}\left(\varpi_{j}\right)_{y} \otimes V^{(t)}\left(\varpi_{i}\right)_{x}, V^{(t)}\left(\varpi_{k}\right)_{z}\right) \neq 0
$$

if and only if there exists a Dynkin quiver $Q$ and $\alpha, \beta, \gamma \in \Phi_{Q}^{+}$such that

(1) $\alpha \prec_{[Q]} \beta$ and $\alpha+\beta=\gamma$,

(2) $V\left(\varpi_{j}\right)_{y}=V_{Q}^{(t)}(\beta)_{a}, V\left(\varpi_{i}\right)_{x}=V_{Q}^{(t)}(\alpha)_{a}, V\left(\varpi_{k}\right)_{z}=V_{Q}^{(t)}(\gamma)_{a}$ for some $a \in \mathbf{k}^{\times}$.

For the rest of this subsection we shall take $\mathrm{g}$ as the simple Lie algebra of type $D_{4}$ when $Q$ is of finite type $D_{4}$ and $g$ as the simple Lie algebra of type $E_{6}$ when $Q$ is of finite type $E_{6}$.

Now, we can apply the same arguments of [KKKO15b, KKKO16] to obtain the following theorem.

Theorem 6.9. For a Dynkin quiver $Q$ of finite type $D_{4}(t=3)$ or $E_{6}(t=2)$, we have the followings:

(1) The functor $\mathcal{F}_{Q}^{(t)}$ sends a simple module to a simple module. Moreover, the functor $\mathcal{F}_{Q}^{(t)}$ induces a bijection from the set of simple modules in $R^{\mathrm{g}}$-gmod to the set of simple modules in $\mathcal{C}_{Q}^{(t)}$.

(2) For any $[Q],\left[Q^{\prime}\right] \in \llbracket \Delta \rrbracket$, there exist the following isomorphisms induced by $\mathcal{F}_{Q}^{(i)}$ and $\mathcal{F}_{Q^{\prime}}^{\left(i^{\prime}\right)}$ :

$$
\begin{cases}{\left.\left[\mathcal{C}_{Q}^{(i)}\right] \simeq U_{\mathbb{A}}^{-}(\mathfrak{g})^{\vee}\right|_{q=1} \simeq\left[\mathcal{C}_{Q^{\prime}}^{\left(i^{\prime}\right)}\right]\left(i, i^{\prime} \in\{1,2,3\}\right)} & \text { if } Q, Q^{\prime} \text { are of type } D_{4}, \\ {\left.\left[\mathcal{C}_{Q}^{(1)}\right] \simeq U_{\mathbb{A}}^{-}(\mathfrak{g})^{\vee}\right|_{q=1} \simeq\left[\mathcal{C}_{Q^{\prime}}^{(2)}\right]} & \text { if } Q, Q^{\prime} \text { are of type } E_{6} .\end{cases}
$$

(3) A dual PBW-basis associated to $[Q]$ and the upper global basis of $U_{\mathbb{A}}^{-}(\mathrm{g})^{\vee}$ are categorified by the modules over $U_{q}^{\prime}\left(\mathfrak{g}^{(t)}\right)$ in the following sense:

(i) The set of simple modules in $\mathcal{C}_{Q}^{(t)}$ corresponds to the upper global basis of $\left.U_{\mathbb{A}}^{-}(\mathrm{g})^{\vee}\right|_{q=1}$.

(ii) The set

$$
\left\{V_{Q}^{(t)}\left(\beta_{1}\right)^{\otimes m_{1}} \otimes \cdots \otimes V_{Q}^{(t)}\left(\beta_{\mathrm{N}}\right)^{\otimes m_{\mathrm{N}}} \mid \underline{m} \in \mathbb{Z}_{\geq 0}^{\mathrm{N}}\right\}
$$

corresponds to the dual PBW-basis associated to $[Q]$ under the isomorphism in (6.8).

(4) The induced ring isomorphisms

$$
\left[\mathcal{F}_{Q}^{\left(i^{\prime}\right)}\right] \circ\left[\mathcal{F}_{Q}^{(i)}\right]^{-1}:\left[\mathcal{C}_{Q}^{(i)}\right] \stackrel{\sim}{\longrightarrow}\left[\mathcal{C}_{Q}^{\left(i^{\prime}\right)}\right]
$$

send simples to simples and preserve dimensions. Here $i, i^{\prime} \in\{1,2,3\}$ if $Q$ is of finite type $D_{4}$, and $i, i^{\prime} \in\{1,2\}$ if $Q$ is of finite type $E_{6}$. 
6.3. $U_{q}^{\prime}\left(F_{4}^{(1)}\right)$ and $U_{q}^{\prime}\left(G_{2}^{(1)}\right)$. In this subsection, we shall prove the $U_{q}^{\prime}\left(F_{4}^{(1)}\right)$ and $U_{q}^{\prime}\left(G_{2}^{(1)}\right)$-analogues of Theorem 3.16, Theorem 3.17 and Theorem 5.13. Since the frameworks for $U_{q}^{\prime}\left(F_{4}^{(1)}\right)$ and $U_{q}^{\prime}\left(G_{2}^{(1)}\right)$ are the same, we shall give proofs for $U_{q}^{\prime}\left(F_{4}^{(1)}\right)$-case only, which is more involved.

Lemma 6.10. For (resp. triply) twisted adapted class $\left[\widetilde{\mathrm{w}}_{0}\right]$ and $\left[\widetilde{\mathrm{w}}_{0}^{\prime}\right]$ of type $A_{2 n-1}$ (resp. $\left.D_{4}\right),(\alpha, \beta)$ of $\gamma \in \Phi^{+}$contained in $\Phi_{\left[\widetilde{w}_{0}\right]}(i, j)(t)$, then there exists a pair $\left(\alpha^{\prime}, \beta^{\prime}\right)$ such that $\alpha^{\prime}+\beta^{\prime} \in \Phi^{+}$and $\left(\alpha^{\prime}, \beta^{\prime}\right)$ is contained in $\Phi_{\left[\widetilde{\mathrm{w}}_{0}^{\prime}\right]}(i, j)(t)$.

Proof. The assertion follows from well-definedness of the distance polynomial $D_{i, j}^{\llbracket \widetilde{\mathrm{w}}_{0} \rrbracket}(z)$ and the fact that $\widehat{\Upsilon}_{\left[\widetilde{w}_{0}^{\prime}\right]}$ can be obtained from $\widehat{\Upsilon}_{\left[\widetilde{\mathrm{w}}_{0}\right]}$ by applying the reflection functors properly.

In this subsection, $U_{q}\left(\mathfrak{g}^{(1)}\right)$ denotes $U_{q}\left(F_{4}^{(1)}\right)$ if $\left[\widetilde{\mathfrak{w}}_{0}\right.$ ] is a twisted adapted class of finite type $E_{6}$, and $U_{q}\left(G_{2}^{(1)}\right)$ if $\left[\widetilde{\mathrm{w}}_{0}\right]$ is a triply twisted adapted class of finite type $D_{4}$.

Proposition 6.11. For a Dynkin diagram $\Delta$ of type $E_{6}$ (resp. $\left.D_{4}\right)$, for any (resp. triply) twisted adapted class $\left[\widetilde{\mathrm{w}}_{0}\right]$, take $J$ and $\mathcal{S}$ as the set of simple roots $\Pi$ associated to $\Delta$. We define two maps

$$
s: \Pi \rightarrow\left\{V^{(1)}\left(\varpi_{i}\right) \mid i \in I_{0}\right\} \quad \text { and } \quad X: \Pi \rightarrow \mathbf{k}^{\times}
$$

as follows: for $\alpha \in \Pi$ with $\widehat{\Omega}_{\left[\widetilde{\mathrm{w}}_{0}\right]}(\alpha)=(i, p / \mathrm{d})$, we define

$$
s(\alpha)=\left\{\begin{array}{ll}
V^{(1)}\left(\varpi_{i^{\star}}\right) & \text { if } \widehat{\Delta} \text { is of type } F_{4}, \\
V^{(1)}\left(\varpi_{i^{\dagger}}\right) & \text { if } \widehat{\Delta} \text { is of type } G_{2},
\end{array} \quad X(\alpha)= \begin{cases}(-1)^{i}\left(-q^{1 / 2}\right)^{p} & \text { if } \widehat{\Delta} \text { is of type } F_{4}, \\
\left(-q^{1 / 3}\right)^{p} & \text { if } \widehat{\Delta} \text { is of type } G_{2} .\end{cases}\right.
$$

Then the underlying graph of $\Gamma^{J}$ coincides with $\Delta$ of finite type $X_{n}$. Hence we have an exact functor

$$
\mathscr{F}_{\left[\widetilde{\mathrm{w}}_{0}\right]}: R^{X_{n}}-\operatorname{gmod} \rightarrow \mathscr{C}_{\left[\widetilde{\mathrm{w}}_{0}\right]}^{(1)} \text {. }
$$

Proof. Note that $\left(\alpha_{i}, \alpha_{j}\right)$ are $\left[\widetilde{\mathrm{w}}_{0}\right]$-minimal if $i, j$ are adjacent in $\Delta$ and $\left[\widetilde{\mathrm{w}}_{0}\right]$-simple, otherwise. Thus the underlying graph of $\Gamma^{J}$ coincides with $\Delta$ by Theorem 4.31 .

Example 6.12. By forgetting the arrows of $\widehat{\Upsilon}_{[\mathscr{Q}]}$ in $(2.10)$, the underlying graph of $\Gamma^{J}$ can be described as follows:

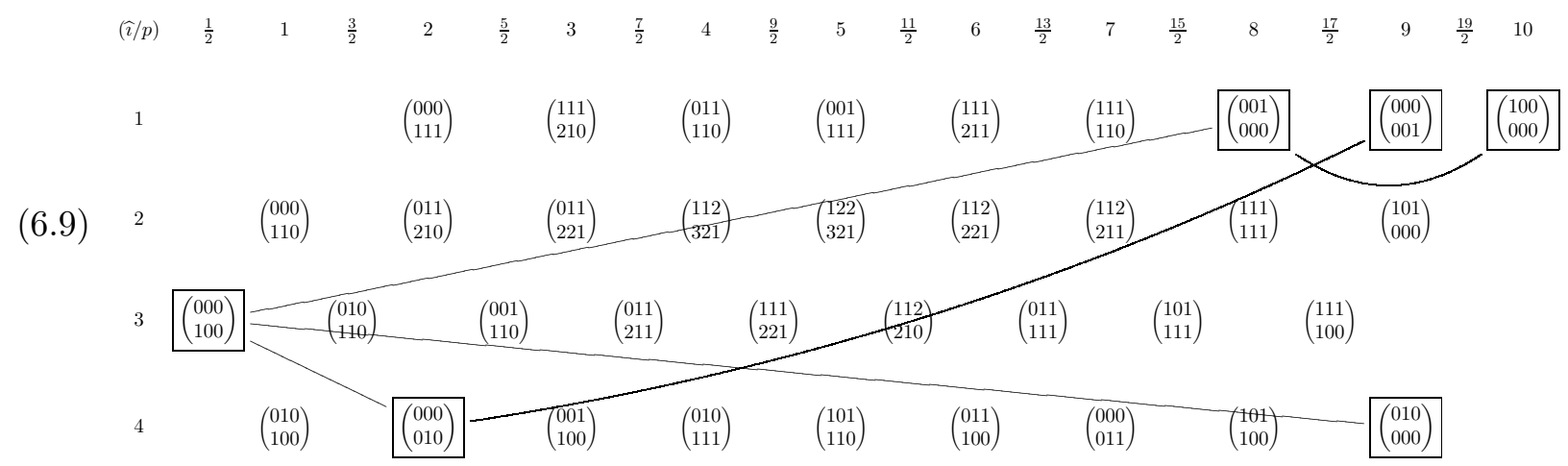


since

$$
\begin{aligned}
& d_{1,1}(z)=\left(z-q_{s}^{4}\right)\left(z-q_{s}^{10}\right)\left(z-q_{s}^{12}\right)\left(z-q_{s}^{18}\right), d_{1,3}(z)=\left(z-q_{s}^{7}\right)\left(z-q_{s}^{9}\right)\left(z-q_{s}^{13}\right)\left(z-q_{s}^{15}\right), \\
& d_{1,4}(z)=\left(z+q_{s}^{8}\right)\left(z+q_{s}^{14}\right), d_{4,4}(z)=\left(z-q_{s}^{2}\right)\left(z-q_{s}^{8}\right)\left(z-q_{s}^{12}\right)\left(z-q_{s}^{18}\right), \\
& d_{3,4}(z)=\left(z+q_{s}^{3}\right)\left(z+q_{s}^{7}\right)\left(z+q_{s}^{9}\right)^{\epsilon^{\prime}}\left(z+q_{s}^{11}\right)\left(z+q_{s}^{13}\right)\left(z+q_{s}^{17}\right) .
\end{aligned}
$$

Theorem 6.13. For any (triply) twisted adapted class $\left[\widetilde{\mathrm{w}}_{0}\right]$ of finite type $X=E_{6}$ or $X=D_{4}$, and $\gamma \in \Phi^{+}$, we have

$$
\mathscr{F}_{\left[\widetilde{\mathrm{w}}_{0}\right]}\left(S_{\left[\widetilde{\mathrm{w}}_{0}\right]}(\gamma)\right) \simeq V_{\left[\widetilde{\mathrm{w}}_{0}\right]}(\gamma) .
$$

At first, we shall prove the above theorem for the folded AR quiver $\widehat{\Upsilon}_{[\mathscr{Q}]}$ in (2.10). By 5.9, we have $\mathscr{F}_{\mathscr{Q}}\left(S_{\mathscr{Q}}\left(\alpha_{i}\right)\right) \simeq V_{\mathscr{Q}}\left(\alpha_{i}\right)$. Hence,

$$
\begin{aligned}
& \mathscr{F}_{\mathscr{Q}}\left(S_{\mathscr{Q}}\left(\alpha_{1}\right)\right) \simeq V\left(\varpi_{1}\right)_{-q_{s}^{20}}, \mathscr{F}_{\mathscr{Q}}\left(S_{\mathscr{Q}}\left(\alpha_{3}\right)\right) \simeq V\left(\varpi_{1}\right)_{-q_{s}^{16}}, \mathscr{F}_{\mathscr{Q}}\left(S_{\mathscr{Q}}\left(\alpha_{6}\right)\right) \simeq V\left(\varpi_{1}\right)_{-q_{s}^{18}}, \\
& \mathscr{F}_{\mathscr{Q}}\left(S_{\mathscr{Q}}\left(\alpha_{2}\right)\right) \simeq V\left(\varpi_{4}\right)_{q_{s}^{18}}, \mathscr{F}_{\mathscr{Q}}\left(S_{\mathscr{Q}}\left(\alpha_{4}\right)\right) \simeq V\left(\varpi_{3}\right)_{-q_{s}^{1}}, \mathscr{F}_{\mathscr{Q}}\left(S_{\mathscr{Q}}\left(\alpha_{5}\right)\right) \simeq V\left(\varpi_{4}\right)_{q_{s}^{4}} .
\end{aligned}
$$

Thus we have an exact sequence

$$
0 \rightarrow S_{\mathscr{Q}}\left(\alpha_{2}+\alpha_{4}\right) \rightarrow S_{\mathscr{Q}}\left(\alpha_{4}\right) \circ S_{\mathscr{Q}}\left(\alpha_{2}\right) \stackrel{\mathbf{r}}{\longrightarrow} S_{\mathscr{Q}}\left(\alpha_{2}\right) \circ S_{\mathscr{Q}}\left(\alpha_{4}\right) \rightarrow S_{\mathscr{Q}}\left(\alpha_{2}+\alpha_{4}\right) \rightarrow 0 .
$$

Applying the exact functor $\mathscr{F}_{\mathscr{Q}}$ to $(6.10)$, we have

$$
\begin{aligned}
0 \rightarrow \mathscr{F}_{\mathscr{Q}}\left(S_{\mathscr{Q}}\left(\alpha_{2}+\alpha_{4}\right)\right) & \rightarrow V\left(\varpi_{4}\right)_{q_{s}^{18}} \otimes V\left(\varpi_{3}\right)_{-q_{s}^{1}} \\
& \stackrel{\mathscr{F}_{\mathscr{Q}}(\mathbf{r})}{\longrightarrow} V\left(\varpi_{3}\right)_{-q_{s}^{1}} \otimes V\left(\varpi_{4}\right)_{q_{s}^{18}} \rightarrow \mathscr{F}_{\mathscr{Q}}\left(S_{\mathscr{Q}}\left(\alpha_{2}+\alpha_{4}\right)\right) \rightarrow 0 .
\end{aligned}
$$

Then (4.32b) implies that

$$
\mathscr{F}_{\mathscr{Q}}\left(S_{\mathscr{Q}}\left(\alpha_{2}+\alpha_{4}\right)\right) \simeq V\left(\varpi_{3}\right)_{-q_{s}^{2}} .
$$

Similarly, we have $\mathscr{F}_{\mathscr{Q}}\left(S_{\mathscr{Q}}\left(\alpha_{1}+\alpha_{3}\right)\right) \simeq V\left(\varpi_{2}\right)_{q_{s}^{18}}$.

Since $\left(\begin{array}{c}000 \\ 010\end{array}\right)$ and $\left(\begin{array}{c}010 \\ 100\end{array}\right)$ is a [Q]-minimal pair for $\left(\begin{array}{c}010 \\ 110\end{array}\right)$, we also have $\mathscr{F}_{\mathscr{Q}}\left(S_{\mathscr{Q}}\left(\begin{array}{c}010 \\ 110\end{array}\right)\right) \simeq V\left(\varpi_{3}\right)_{-q_{s}^{3}}$.

Note that we have an exact sequence

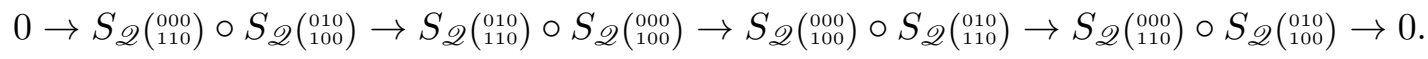

where $S_{\mathscr{Q}}\left(\begin{array}{c}000 \\ 110\end{array}\right) \circ S_{\mathscr{Q}}\left(\begin{array}{c}010 \\ 100\end{array}\right)$ is simple.

By applying the exact functor $\mathscr{F}_{\mathscr{Q}}$ to $(6.11)$, we have

$$
\begin{aligned}
0 \rightarrow \mathscr{F}_{\mathscr{Q}}\left(S_{\mathscr{Q}}\left(\begin{array}{c}
000 \\
110
\end{array}\right)\right) & \otimes V\left(\varpi_{4}\right)_{q_{s}^{2}} \rightarrow V\left(\varpi_{3}\right)_{-q_{s}^{3}} \otimes V\left(\varpi_{3}\right)_{-q_{s}^{1}} \\
& \rightarrow V\left(\varpi_{3}\right)_{-q_{s}^{1}} \otimes V\left(\varpi_{3}\right)_{-q_{s}^{3}} \rightarrow V\left(\varpi_{4}\right)_{q_{s}^{2}} \otimes \mathscr{F}_{\mathscr{Q}}\left(S_{\mathscr{Q}}\left(\begin{array}{l}
(000 \\
110
\end{array}\right)\right) \rightarrow 0 .
\end{aligned}
$$
have

By [Her06, Page 23], $V\left(\varpi_{3}\right)_{-q_{s}^{1}} \otimes V\left(\varpi_{3}\right)_{-q_{s}^{3}}$ has a simple head $V\left(\varpi_{4}\right)_{q_{s}^{2}} \otimes V\left(\varpi_{2}\right)_{q_{s}^{2}}$. Hence we

by the same argument in the previous subsection.

$$
\mathscr{F}_{\mathscr{Q}}\left(S_{\mathscr{Q}}\left(\begin{array}{c}
000 \\
110
\end{array}\right)\right) \simeq V\left(\varpi_{2}\right)_{q_{s}^{2}}
$$

Since $\left(\begin{array}{l}000 \\ 010\end{array}\right)$ and $\left(\begin{array}{l}000 \\ 100\end{array}\right)$ is another [Q]-minimal pair for $\left(\begin{array}{c}000 \\ 110\end{array}\right)$, we have

$$
V\left(\varpi_{3}\right)_{-q_{s}^{1}} \otimes V\left(\varpi_{4}\right)_{q_{s}^{4}} \rightarrow V\left(\varpi_{2}\right)_{q_{s}^{2}} .
$$

Now we have the following results with the Dorey's type homomorphisms (4.32a), (4.32b), (4.32c) and $(4.32 \mathrm{~d})$ sequentially:

- $\left(\left(\begin{array}{c}000 \\ 001\end{array}\right),\left(\begin{array}{c}000 \\ 110\end{array}\right)\right)$ is a $[\mathscr{Q}]$-minimal pair of $\left(\begin{array}{c}000 \\ 111\end{array}\right),(4.32 \mathrm{a})$ implies $\mathscr{F}_{\mathscr{Q}}\left(S_{\mathscr{Q}}\left(\begin{array}{c}000 \\ 111\end{array}\right)\right) \simeq V\left(\varpi_{1}\right)_{-q_{s}^{4}}$, 
- $\left(\left(\begin{array}{c}000 \\ 001\end{array}\right),\left(\begin{array}{c}000 \\ 010\end{array}\right)\right)$ is a $[\mathscr{Q}]$-minimal pair of $\left(\begin{array}{c}000 \\ 011\end{array}\right),(4.32 \mathrm{~d})$ implies $\mathscr{F}_{\mathscr{Q}}\left(S_{\mathscr{Q}}\left(\begin{array}{c}000 \\ 011\end{array}\right)\right) \simeq V\left(\varpi_{4}\right)_{q_{s}^{14}}$,

- $\left(\left(\begin{array}{c}001 \\ 000\end{array}\right),\left(\begin{array}{c}010 \\ 100\end{array}\right)\right)$ is a $[\mathscr{Q}]$-minimal pair of $\left(\begin{array}{c}011 \\ 100\end{array}\right),(4.32 \mathrm{~d})$ implies $\mathscr{F}_{\mathscr{Q}}\left(S_{\mathscr{Q}}\left(\begin{array}{c}000 \\ 011\end{array}\right)\right) \simeq V\left(\varpi_{4}\right)_{q_{s}^{12}}$,

- $\left(\left(\begin{array}{c}000 \\ 011\end{array}\right),\left(\begin{array}{l}011 \\ 100\end{array}\right)\right)$ is a [Q]-minimal pair of $\left(\begin{array}{c}011 \\ 111\end{array}\right),(4.32 \mathrm{~b})$ implies $\mathscr{F}_{\mathscr{Q}}\left(S_{\mathscr{Q}}\left(\begin{array}{c}011 \\ 111\end{array}\right)\right) \simeq V\left(\varpi_{3}\right)_{-q_{s}^{13}}$,

- $\left(\left(\begin{array}{l}011 \\ 100\end{array}\right),\left(\begin{array}{c}000 \\ 010\end{array}\right)\right)$ is a [Q]-minimal pair of $\left(\begin{array}{c}011 \\ 110\end{array}\right),(4.32 \mathrm{~d})$ implies $\mathscr{F}_{\mathscr{Q}}\left(S_{\mathscr{Q}}\left(\begin{array}{c}011 \\ 110\end{array}\right)\right) \simeq V\left(\varpi_{1}\right)_{-q_{s}^{18}}$,

- $\left(\left(\begin{array}{c}000 \\ 011\end{array}\right),\left(\begin{array}{c}010 \\ 100\end{array}\right)\right)$ is a [Q]-minimal pair of $\left(\begin{array}{c}010 \\ 111\end{array}\right),(4.32 \mathrm{c})$ implies $\mathscr{F}_{\mathscr{Q}}\left(S_{\mathscr{Q}}\left(\begin{array}{l}010 \\ 111\end{array}\right)\right) \simeq V\left(\varpi_{4}\right)_{q_{s}^{8}}$.

Since $\left(\begin{array}{l}000 \\ 001\end{array}\right)$ and $\left(\begin{array}{l}010 \\ 110\end{array}\right)$ is another $[\mathscr{Q}]$-minimal pair for $\left(\begin{array}{c}010 \\ 111\end{array}\right)$, we have

$$
V\left(\varpi_{3}\right)_{-q_{s}^{3}} \otimes V\left(\varpi_{1}\right)_{-q_{s}^{18}} \rightarrow V\left(\varpi_{4}\right)_{q_{s}^{8}} .
$$

Then we have the followings

- $\left(\left(\begin{array}{c}000 \\ 100\end{array}\right),\left(\begin{array}{c}001 \\ 000\end{array}\right)\right)$ is a [Q2]-minimal pair of $\left(\begin{array}{c}001 \\ 100\end{array}\right),(6.13)$ implies $\mathscr{F}_{\mathscr{Q}}\left(S_{\mathscr{Q}}\left(\begin{array}{l}001 \\ 100\end{array}\right)\right) \simeq V\left(\varpi_{4}\right)_{q_{s}^{6}}$,

- $\left(\left(\begin{array}{c}001 \\ 100\end{array}\right),\left(\begin{array}{c}000 \\ 010\end{array}\right)\right)$ is a [Q]-minimal pair of $\left(\begin{array}{c}001 \\ 110\end{array}\right),(4.32 \mathrm{~b})$ implies $\mathscr{F}_{\mathscr{Q}}\left(S_{\mathscr{Q}}\left(\begin{array}{c}001 \\ 110\end{array}\right)\right) \simeq V\left(\varpi_{3}\right)_{-q_{s}^{5}}$,

- $\left(\left(\begin{array}{c}010 \\ 111\end{array}\right),\left(\begin{array}{c}001 \\ 100\end{array}\right)\right)$ is a [Q]-minimal pair of $\left(\begin{array}{c}011 \\ 211\end{array}\right),(4.32 \mathrm{~b})$ implies $\mathscr{F}_{\mathscr{Q}}\left(S_{\mathscr{Q}}\left(\begin{array}{c}011 \\ 211\end{array}\right)\right) \simeq V\left(\varpi_{3}\right)_{-q_{s}^{7}}$,

- $\left(\left(\begin{array}{l}001 \\ 100\end{array}\right),\left(\begin{array}{l}010 \\ 110\end{array}\right)\right)$ is a [Q]-minimal pair of $\left(\begin{array}{c}011 \\ 210\end{array}\right),(6.12)$ implies $\mathscr{F}_{\mathscr{Q}}\left(S_{\mathscr{Q}}\left(\begin{array}{l}011 \\ 210\end{array}\right)\right) \simeq V\left(\varpi_{2}\right)_{q_{s}^{4}}$,

- $\left(\left(\begin{array}{c}010 \\ 111\end{array}\right),\left(\begin{array}{l}001 \\ 110\end{array}\right)\right)$ is a $[\mathscr{Q}]$-minimal pair of $\left(\begin{array}{c}011 \\ 221\end{array}\right),(6.12)$ implies $\mathscr{F}_{\mathscr{Q}}\left(S_{\mathscr{Q}}\left(\begin{array}{l}011 \\ 221\end{array}\right)\right) \simeq V\left(\varpi_{2}\right)_{q_{s}^{6}}$,

- $\left(\left(\begin{array}{c}000 \\ 011\end{array}\right),\left(\begin{array}{c}001 \\ 100\end{array}\right)\right)$ is a [Q]-minimal pair of $\left(\begin{array}{c}001 \\ 111\end{array}\right),(4.32 \mathrm{~d})$ implies $\mathscr{F}_{\mathscr{Q}}\left(S_{\mathscr{Q}}\left(\begin{array}{c}001 \\ 111\end{array}\right)\right) \simeq V\left(\varpi_{1}\right)_{-q_{s}^{10}}$,

- $\left(\left(\begin{array}{c}001 \\ 110\end{array}\right),\left(\begin{array}{c}100 \\ 000\end{array}\right)\right)$ is a [Q]-minimal pair of $\left(\begin{array}{c}101 \\ 110\end{array}\right),(6.13)$ implies $\mathscr{F}_{\mathscr{Q}}\left(S_{\mathscr{Q}}\left(\begin{array}{l}101 \\ 110\end{array}\right)\right) \simeq V\left(\varpi_{4}\right)_{q_{s}^{10}}$,

- $\left(\left(\begin{array}{l}100 \\ 000\end{array}\right),\left(\begin{array}{c}001 \\ 100\end{array}\right)\right)$ is a $[\mathscr{Q}]$-minimal pair of $\left(\begin{array}{l}101 \\ 100\end{array}\right),(4.32 \mathrm{~d})$ implies $\mathscr{F}_{\mathscr{Q}}\left(S_{\mathscr{Q}}\left(\begin{array}{c}101 \\ 100\end{array}\right)\right) \simeq V\left(\varpi_{4}\right)_{q_{s}^{16}}$,

sequentially.

Now we can say that

(a) $\mathscr{F}_{\mathscr{Q}}\left(S_{\mathscr{Q}}(\beta)\right) \simeq V_{\mathscr{Q}}(\beta)$ for all $\beta \in \Phi^{+}$by applying the above arguments,

(b) $V_{\mathscr{Q}}(\beta) \otimes V_{\mathscr{Q}}(\alpha) \rightarrow V_{\mathscr{Q}}(\gamma)$ for every [Q2]-minimal pair $(\alpha, \beta)$ of $\gamma \in \Phi^{+}$.

Proof of theorem 6.13. We have shown that our assertion holds for the [Q2]. By Lemma 6.10, we can apply the induction argument on $|\gamma|$ for any [Q2] of finite type $E_{6}$.

Corollary 6.14. For $U_{q}^{\prime}\left(F_{4}^{(1)}\right)\left(X=E_{6}\right)$ or $U_{q}^{\prime}\left(G_{2}^{(1)}\right)\left(X=D_{4}\right)$, let $(i, x),(j, y),(k, z) \in I_{0} \times \mathbf{k}^{\times}$. Then

$$
\operatorname{dim}_{\mathbf{k}}\left(\operatorname{Hom}_{U_{q}^{\prime}\left(\mathfrak{g}^{(1)}\right)}\left(V^{(t)}\left(\varpi_{j}\right)_{y} \otimes V^{(t)}\left(\varpi_{i}\right)_{x}, V^{(t)}\left(\varpi_{k}\right)_{z}\right)\right)=1
$$

if and only if there exists a (triply) twisted adapted class $\left[\widetilde{\mathrm{w}}_{0}\right]$ and $\alpha, \beta, \gamma \in \Phi_{X}^{+}$such that

(1) $(\alpha, \beta)$ is a $\left[\widetilde{\mathrm{w}}_{0}\right]$-minimal pair of $\gamma$,

(2) $V\left(\varpi_{j}\right)_{y}=V_{\mathscr{Q}}(\beta)_{a}, V\left(\varpi_{i}\right)_{x}=V_{\mathscr{Q}}(\alpha)_{a}, V\left(\varpi_{k}\right)_{z}=V_{\mathscr{Q}}(\gamma)_{a}$ for some $a \in \mathbf{k}^{\times}$.

For the rest of this subsection we shall take $g$ as the simple Lie algebra of type $E_{6}$ when $\left[\widetilde{\mathrm{w}}_{0}\right]$ is a twisted adapted class of finite type $E_{6}$ and $\mathrm{g}$ as the simple Lie algebra of type $D_{4}$ when $\left[\widetilde{\mathrm{w}}_{0}\right.$ ] is a triply twisted adapted class of finite type $D_{4}$.

Now, we can apply the same arguments of [KKKO15b, KKKO16, KO18] to obtain the following theorem.

Theorem 6.15. For a (triply) twisted adapted class $\left[\widetilde{\mathrm{w}}_{0}\right]=[\mathscr{Q}]$ of finite type $E_{6}$ or $\left[\widetilde{\mathrm{w}}_{0}\right]=[\mathfrak{Q}]$ of finite type $D_{4}$, we have the followings:

(1) The functor $\mathscr{F}_{\left[\widetilde{w}_{0}\right]}$ sends a simple module to a simple module. Moreover, the functor $\mathscr{F}_{\left[\widetilde{w}_{0}\right]}$ induces a bijection from the set of simple modules in $R^{\mathrm{g}}$-gmod to the set of simple modules in $\mathscr{C}_{\left[\tilde{\mathrm{w}}_{0}\right]}^{(1)}$. 
(2) For any $\left[\widetilde{\mathrm{w}}_{0}\right] \in \llbracket \widetilde{\mathrm{w}}_{0} \rrbracket$, there exist isomorphisms induced by the functors $\mathcal{F}_{Q}^{(i)}, \mathscr{F}_{\mathscr{Q}}$ and $\mathscr{F}_{\mathfrak{Q}}$ :
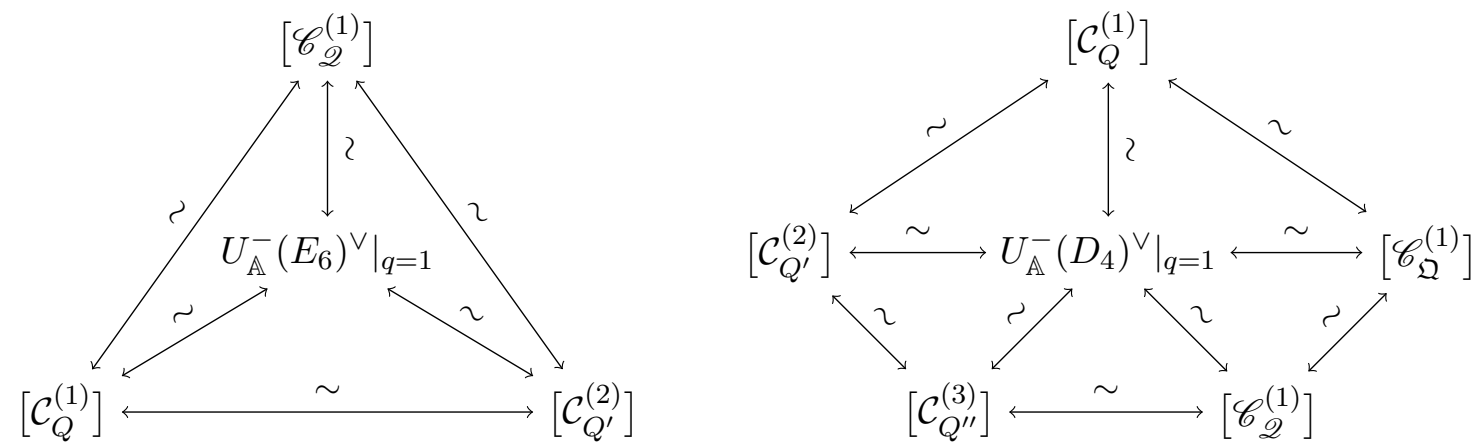

(3) A dual PBW-basis associated to $\left[\widetilde{\mathrm{w}}_{0}\right]$ and the upper global basis of $U_{\mathbb{A}}^{-}(\mathrm{g})^{\vee}$ are categorified by the modules over $U_{q}^{\prime}(\mathfrak{g})$ in the following sense:

(i) The set of simple modules in $\mathscr{C}_{\left[\widetilde{\mathrm{w}}_{0}\right]}^{(1)}$ corresponds to the upper global basis of $\left.U_{\mathbb{A}}^{-}(\mathrm{g})^{\vee}\right|_{q=1}$.

(ii) The set

$$
\left\{V_{\left[\widetilde{\mathrm{w}}_{0}\right]}\left(\beta_{1}\right)^{\otimes m_{1}} \otimes \cdots \otimes V_{\left[\widetilde{\mathrm{w}}_{0}\right]}\left(\beta_{\mathrm{N}}\right)^{\otimes m_{\mathrm{N}}} \mid \underline{m} \in \mathbb{Z}_{\geq 0}^{\mathrm{N}}\right\}
$$

corresponds to the dual PBW-basis associated to $\left[\widetilde{\mathrm{w}}_{0}\right]$ under the isomorphism in (6.8).

(4) Any induced ring isomorphisms in (2) send simples to simples.

\section{REFINING DENOMINATOR FORMULAS}

As we appointed, we shall refined the denominator formulas for $U_{q}^{\prime}\left(F_{4}^{(1)}\right)$ and $U_{q}^{\prime}\left(E_{6}^{(2)}\right)$ by using the generalized Schur-Weyl duality functor for them, investigated in the previous section.

7.1. $U_{q}^{\prime}\left(F_{4}^{(1)}\right)$. To refine the denominator formulas for $U_{q}^{\prime}\left(F_{4}^{(1)}\right)$, we shall use $\widehat{\Upsilon}_{\mathscr{Q}}$ in $(2.10)$.

Proposition 7.1. We can refine $d_{3,4}(z)$ as follows:

$$
d_{3,4}^{F_{4}^{(1)}}(z)=\left(z+q_{s}^{3}\right)\left(z+q_{s}^{7}\right)\left(z+q_{s}^{11}\right)\left(z+q_{s}^{13}\right)\left(z+q_{s}^{17}\right)
$$

Hence we have

$$
d_{3,3}^{F_{4}^{(1)}}(z)=\left(z-q_{s}^{2}\right)\left(z-q_{s}^{6}\right)\left(z-q_{s}^{8}\right)\left(z-q_{s}^{10}\right)\left(z-q_{s}^{12}\right)^{2}\left(z-q_{s}^{16}\right)\left(z-q_{s}^{18}\right) .
$$

Proof. Note that the pair $\left(\left(\begin{array}{c}000 \\ 100\end{array}\right),\left(\begin{array}{l}101 \\ 110\end{array}\right)\right)$ is [Q $]$-simple and hence $S_{\mathscr{Q}}\left(\begin{array}{c}000 \\ 100\end{array}\right) \circ S_{\mathscr{Q}}\left(\begin{array}{c}101 \\ 110\end{array}\right)$ is simple. By Theorem 6.15, $V\left(\varpi_{3}\right)_{-q_{s}^{1}} \otimes V\left(\varpi_{4}\right)_{-q_{s}^{10}}$ is also simple and hence our assertion follows.

Proposition 7.2. We can refine $d_{1,3}(z)$ as follows:

$$
d_{1,3}^{F_{4}^{(1)}}(z)=\left(z-q_{s}^{7}\right)\left(z-q_{s}^{9}\right)\left(z-q_{s}^{13}\right)\left(z-q_{s}^{15}\right) .
$$

Proof. Note that we have $S_{\mathscr{Q}}\left(\begin{array}{l}101 \\ 100\end{array}\right) \hookrightarrow S_{\mathscr{Q}}\left(\begin{array}{c}100 \\ 000\end{array}\right) \circ S_{\mathscr{Q}}\left(\begin{array}{c}001 \\ 100\end{array}\right)$, and $S_{\mathscr{Q}}\left(\begin{array}{c}001 \\ 100\end{array}\right) \circ S_{\mathscr{Q}}\left(\begin{array}{l}111 \\ 110\end{array}\right) \rightarrow S_{\mathscr{Q}}\left(\begin{array}{c}112 \\ 210\end{array}\right)$. Then we have

$$
S_{\mathscr{Q}}\left(\begin{array}{l}
101 \\
100
\end{array}\right) \circ S_{\mathscr{Q}}\left(\begin{array}{l}
111 \\
110
\end{array}\right) \hookrightarrow S_{\mathscr{Q}}\left(\begin{array}{l}
100 \\
000
\end{array}\right) \circ S_{\mathscr{Q}}\left(\begin{array}{l}
001 \\
100
\end{array}\right) \circ S_{\mathscr{Q}}\left(\begin{array}{l}
111 \\
110
\end{array}\right) \rightarrow S_{\mathscr{Q}}\left(\begin{array}{l}
100 \\
000
\end{array}\right) \circ S_{\mathscr{Q}}\left(\begin{array}{l}
112 \\
210
\end{array}\right)
$$

where the composition is non-zero by Proposition 5.7. Hence $S_{\mathscr{Q}}\left(\begin{array}{l}100 \\ 0000\end{array}\right) \circ S_{\mathscr{Q}}\left(\begin{array}{l}112 \\ 210\end{array}\right)$ is not simple by Theorem 5.8. By applying the functor $\mathscr{F}_{\mathscr{Q}}$, we can say that $q_{s}^{9}$ is a root of $d_{1,3}(z)$ by Theorem 3.10. 
Proposition 7.3. We have

$$
d_{2,3}^{F_{4}^{(1)}}(z)=\left(z+q_{s}^{5}\right)\left(z+q_{s}^{7}\right)\left(z+q_{s}^{9}\right)\left(z+q_{s}^{11}\right)^{2}\left(z+q_{s}^{13}\right)\left(z+q_{s}^{15}\right)\left(z+q_{s}^{17}\right) .
$$

Proof. Note that we have $S_{\mathscr{Q}}\left(\begin{array}{c}000 \\ 111\end{array}\right) \hookrightarrow S_{\mathscr{Q}}\left(\begin{array}{c}000 \\ 001\end{array}\right) \circ S_{\mathscr{Q}}\left(\begin{array}{c}000 \\ 110\end{array}\right), \quad S_{\mathscr{Q}}\left(\begin{array}{c}000 \\ 010\end{array}\right) \circ S_{\mathscr{Q}}\left(\begin{array}{c}000 \\ 001\end{array}\right) \rightarrow S_{\mathscr{Q}}\left(\begin{array}{c}000 \\ 011\end{array}\right)$. Then we have

$$
S_{\mathscr{Q}}\left(\begin{array}{c}
000 \\
010
\end{array}\right) \circ S_{\mathscr{Q}}\left(\begin{array}{c}
000 \\
111
\end{array}\right) \hookrightarrow S_{\mathscr{Q}}\left(\begin{array}{c}
000 \\
010
\end{array}\right) \circ S_{\mathscr{Q}}\left(\begin{array}{c}
000 \\
001
\end{array}\right) \circ S_{\mathscr{Q}}\left(\begin{array}{c}
000 \\
110
\end{array}\right) \rightarrow S_{\mathscr{Q}}\left(\begin{array}{c}
000 \\
011
\end{array}\right) \circ S_{\mathscr{Q}}\left(\begin{array}{c}
000 \\
110
\end{array}\right)
$$

whose composition is non-zero by Proposition 5.7 .

Similarly, we have the homomorphisms $S_{\mathscr{Q}}\left(\begin{array}{l}111 \\ 221\end{array}\right) \hookrightarrow S_{\mathscr{Q}}^{\left(\begin{array}{c}000 \\ 011\end{array}\right)} \circ S_{\mathscr{Q}}\left(\begin{array}{l}111 \\ 210\end{array}\right)$. Then we have

$$
S_{\mathscr{Q}}\left(\begin{array}{l}
000 \\
110
\end{array}\right) \circ S_{\mathscr{Q}}\left(\begin{array}{l}
111 \\
221
\end{array}\right) \hookrightarrow S_{\mathscr{Q}}\left(\begin{array}{l}
000 \\
110
\end{array}\right) \circ S_{\mathscr{Q}}\left(\begin{array}{l}
000 \\
011
\end{array}\right) \circ S_{\mathscr{Q}}\left(\begin{array}{l}
111 \\
210
\end{array}\right) \rightarrow S_{\mathscr{Q}}\left(\begin{array}{l}
000 \\
111
\end{array}\right) \circ S_{\mathscr{Q}}\left(\begin{array}{c}
000 \\
010
\end{array}\right) \circ S_{\mathscr{Q}}\left(\begin{array}{l}
111 \\
210
\end{array}\right)
$$

where the composition is non-zero by Proposition 5.7 again.

Since $S_{\mathscr{Q}}\left(\begin{array}{l}000 \\ 111\end{array}\right) \circ S_{\mathscr{Q}}\left(\begin{array}{c}000 \\ 010\end{array}\right) \circ S_{\mathscr{Q}}\left(\begin{array}{l}111 \\ 210\end{array}\right)$ is simple, we can conclude that $S_{\mathscr{Q}}\left(\begin{array}{c}000 \\ 110\end{array}\right) \circ S_{\mathscr{Q}}\left(\begin{array}{l}111 \\ 221\end{array}\right)$ is not simple by Theorem 5.8. By applying the functor $\mathscr{F}_{\mathscr{Q}}$, we can say that $-q_{s}^{7}$ is a root of $d_{2,3}(z)$.

Proposition 7.4. We have

$$
d_{2,2}^{F_{4}^{(1)}}(z)=\left(z-q_{s}^{4}\right)\left(z-q_{s}^{6}\right)\left(z-q_{s}^{8}\right)^{2}\left(z-q_{s}^{10}\right)^{2}\left(z-q_{s}^{12}\right)^{2}\left(z-q_{s}^{14}\right)^{2}\left(z-q_{s}^{16}\right)\left(z-q_{s}^{18}\right) .
$$

Proof. Assume the order of the root $q_{s}^{8}$ is 1. Then, by Lemma 3.7, the composition length of $V_{\mathscr{Q}}\left(\begin{array}{c}000 \\ 110\end{array}\right) \otimes V_{\mathscr{Q}}\left(\begin{array}{c}122 \\ 321\end{array}\right)$ is 2 . By applying the functor $\mathscr{F}_{\mathscr{Q}}$, the composition length of $S_{\mathscr{Q}}\left(\begin{array}{c}000 \\ 110\end{array}\right) \circ S_{\mathscr{Q}}\left(\begin{array}{l}122 \\ 321\end{array}\right)$ is 2 , too.

Applying the same argument of Proposition 7.3, we have the following non-zero homomorphisms:

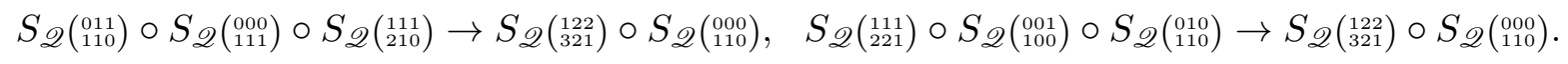

Thus the composition length of $S_{\mathscr{Q}}\left(\begin{array}{l}000 \\ 110\end{array}\right) \circ S_{\mathscr{Q}}\left(\begin{array}{l}122 \\ 321\end{array}\right)$ can not be 2 by Theorem 5.8 .

7.2. $U_{q}^{\prime}\left(E_{6}^{(2)}\right)$. To refine the denominator formulas for $U_{q}^{\prime}\left(E_{6}^{(2)}\right)$, we shall use $\Gamma_{Q}$ in (2.6).

Proposition 7.5. We have

$$
d_{2,2}^{E_{6}^{(2)}}(z)=\left(z-q^{2}\right)\left(z-q^{4}\right)\left(z-q^{6}\right)\left(z-q^{8}\right)^{2}\left(z-q^{10}\right)\left(z+q^{4}\right)\left(z+q^{6}\right)^{2}\left(z+q^{8}\right)\left(z+q^{10}\right)\left(z+q^{12}\right) .
$$

Proof. Note that we have $S_{Q}\left(\begin{array}{c}000 \\ 011\end{array}\right) \hookrightarrow S_{Q}\left(\begin{array}{c}000 \\ 010\end{array}\right) \circ S_{Q}\left(\begin{array}{c}000 \\ 001\end{array}\right), \quad S_{Q}\left(\begin{array}{c}000 \\ 001\end{array}\right) \circ S_{Q}\left(\begin{array}{c}010 \\ 110\end{array}\right) \rightarrow S_{Q}\left(\begin{array}{c}010 \\ 111\end{array}\right)$. Then we have

$$
S_{Q}\left(\begin{array}{c}
000 \\
011
\end{array}\right) \circ S_{Q}\left(\begin{array}{c}
010 \\
110
\end{array}\right) \hookrightarrow S_{Q}\left(\begin{array}{c}
000 \\
010
\end{array}\right) \circ S_{Q}\left(\begin{array}{c}
000 \\
001
\end{array}\right) \circ S_{Q}\left(\begin{array}{c}
010 \\
110
\end{array}\right) \rightarrow S_{Q}\left(\begin{array}{c}
000 \\
010
\end{array}\right) \circ S_{Q}\left(\begin{array}{l}
010 \\
111
\end{array}\right) .
$$

where the composition is non-zero by Proposition 5.7. Since $S_{Q}\left(\begin{array}{l}111 \\ 221\end{array}\right) \hookrightarrow S_{Q}^{\left(\begin{array}{c}010 \\ 110\end{array}\right)} \circ S_{Q}\left(\begin{array}{l}101 \\ 111\end{array}\right)$, we have

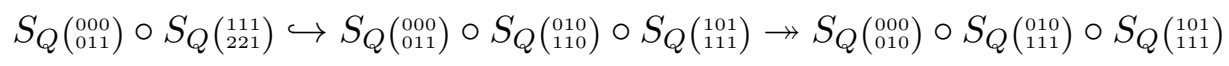

where the composition is non-zero by Proposition 5.7, again. By applying the functor $\mathcal{F}_{Q}^{(2)}$, we can say that $-q^{4}$ is a root of $d_{2,2}(z)$ by Theorem 3.10 .

Proposition 7.6. We have

$$
d_{2,3}^{E_{6}^{(2)}}(z)=\left(z^{2}+q^{6}\right)\left(z^{2}+q^{10}\right)^{2}\left(z^{2}+q^{14}\right)^{2}\left(z^{2}+q^{18}\right)^{2}\left(z^{2}+q^{22}\right) .
$$


Proof. Assume the root $\sqrt{-1} q^{5}$ is of order 1. Then, by Lemma 3.7, the composition length of $V_{Q}\left(\begin{array}{c}112 \\ 321\end{array}\right) \otimes V_{Q}\left(\begin{array}{c}000 \\ 011\end{array}\right)$ is 2 . By Theorem 6.9, the composition length of $S_{Q}\left(\begin{array}{l}112 \\ 321\end{array}\right) \circ S_{Q}\left(\begin{array}{c}000 \\ 011\end{array}\right)$ is 2 , too.

In a similar way of the proof of the previous proposition, we have non-zero homomorphisms

$$
\begin{aligned}
& S_{Q}\left(\begin{array}{c}
000 \\
011
\end{array}\right) \circ S_{Q}\left(\begin{array}{c}
112 \\
321
\end{array}\right) \hookrightarrow S_{Q}\left(\begin{array}{c}
000 \\
011
\end{array}\right) \circ S_{Q}\left(\begin{array}{c}
101 \\
110
\end{array}\right) \circ S_{Q}\left(\begin{array}{l}
011 \\
211
\end{array}\right) \rightarrow S_{Q}\left(\begin{array}{c}
000 \\
010
\end{array}\right) \circ S_{Q}\left(\begin{array}{c}
101 \\
111
\end{array}\right) \circ S_{Q}\left(\begin{array}{l}
011 \\
211
\end{array}\right), \\
& S_{Q}\left(\begin{array}{c}
000 \\
011
\end{array}\right) \circ S_{Q}\left(\begin{array}{l}
112 \\
321
\end{array}\right) \hookrightarrow S_{Q}\left(\begin{array}{c}
000 \\
011
\end{array}\right) \circ S_{Q}\left(\begin{array}{c}
001 \\
100
\end{array}\right) \circ S_{Q}\left(\begin{array}{l}
111 \\
221
\end{array}\right) \rightarrow S_{Q}\left(\begin{array}{l}
001 \\
111
\end{array}\right) \circ S_{Q}\left(\begin{array}{l}
111 \\
221
\end{array}\right) .
\end{aligned}
$$

By taking dual, one can prove that the composition length of $S_{Q}\left(\begin{array}{l}112 \\ 321\end{array}\right) \circ S_{Q}\left(\begin{array}{c}000 \\ 011\end{array}\right)$ can not be 2 by Theorem 5.8.

Proposition 7.7. We have

$$
d_{3,4}^{E_{6}^{(2)}}(z)=\left(z^{2}-q^{6}\right)\left(z^{2}-q^{10}\right)\left(z^{2}-q^{14}\right)^{2}\left(z^{2}-q^{18}\right)^{2}\left(z^{2}-q^{22}\right) .
$$

Proof. Assume that $q^{3}$ is not a root of $d_{3,4}(z)$. Theorem 3.10 tells that $V_{Q}\left(\begin{array}{l}\text { ooo } \\ 111\end{array}\right) \otimes V_{Q}\left(\begin{array}{l}\text { ool } \\ 110\end{array}\right)$ is simple. By Theorem 6.9, it implies that $S_{Q}\left(\begin{array}{l}000 \\ 111\end{array}\right) \circ S_{Q}\left(\begin{array}{l}001 \\ 110\end{array}\right)$ is simple, too.

However, we can obtain a non-zero homomorphism

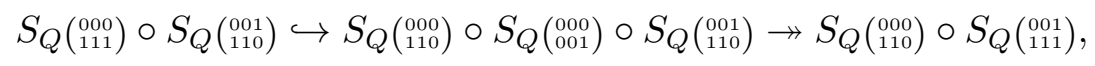

which yields contradiction by Theorem 5.8 .

Proposition 7.8. We can refine $d_{3,3}(z)$ as follows: For some $\epsilon^{\prime} \in\{0,1\}$, we have

$$
d_{3,3}(z)=\left(z^{2}-q^{4}\right)\left(z^{2}-q^{8}\right)^{2}\left(z^{2}-q^{12}\right)^{2+\epsilon^{\prime}}\left(z^{2}-q^{16}\right)^{3}\left(z^{2}-q^{20}\right)^{2}\left(z^{2}-q^{24}\right) .
$$

Proof. Assume the order of the root $q^{4}$ is 1 . Then, by Lemma 3.7, the composition length of $V_{Q}\left(\begin{array}{l}000 \\ 111\end{array}\right) \otimes V_{Q}\left(\begin{array}{c}112 \\ 321\end{array}\right)$ is 2. By Theorem 6.9, the composition length of $S_{Q}\left(\begin{array}{c}000 \\ 111\end{array}\right) \circ S_{Q}\left(\begin{array}{c}112 \\ 321\end{array}\right)$ is 2 , too.

In a similar way of the proof of the previous proposition, we have non-zero homomorphisms

$$
\begin{aligned}
& S_{Q}\left(\begin{array}{c}
000 \\
111
\end{array}\right) \circ S_{Q}\left(\begin{array}{l}
112 \\
321
\end{array}\right) \hookrightarrow S_{Q}\left(\begin{array}{c}
000 \\
111
\end{array}\right) \circ S_{Q}\left(\begin{array}{c}
101 \\
110
\end{array}\right) \circ S_{Q}\left(\begin{array}{l}
011 \\
211
\end{array}\right) \rightarrow S_{Q}\left(\begin{array}{c}
000 \\
110
\end{array}\right) \circ S_{Q}\left(\begin{array}{l}
101 \\
111
\end{array}\right) \circ S_{Q}\left(\begin{array}{l}
011 \\
211
\end{array}\right), \\
& S_{Q}\left(\begin{array}{c}
000 \\
111
\end{array}\right) \circ S_{Q}\left(\begin{array}{l}
112 \\
321
\end{array}\right) \hookrightarrow S_{Q}\left(\begin{array}{c}
000 \\
111
\end{array}\right) \circ S_{Q}\left(\begin{array}{c}
001 \\
100
\end{array}\right) \circ S_{Q}\left(\begin{array}{l}
111 \\
221
\end{array}\right) \rightarrow S_{Q}\left(\begin{array}{l}
000 \\
100
\end{array}\right) \circ S_{Q}\left(\begin{array}{c}
001 \\
111
\end{array}\right) \circ S_{Q}\left(\begin{array}{l}
211 \\
231
\end{array}\right) .
\end{aligned}
$$

By taking duals, one can notice that $S_{Q}\left(\begin{array}{c}000 \\ 111\end{array}\right) \circ S_{Q}\left(\begin{array}{c}112 \\ 321\end{array}\right)$ cannot be 2 by Theorem 5.8 .

The $\epsilon^{\prime}$ is indeed 1, which is proved in an addendum to our publication [OS19] (see Appendix E).

\section{Appendix A. Dynkin quiver $Q$ of type $E_{7}$ And its AR Quiver $\Gamma_{Q}$}

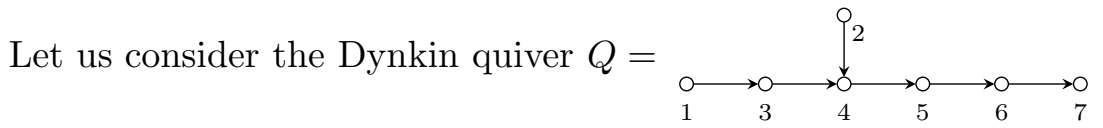

Its $\mathrm{AR}$ quiver $\Gamma_{Q}$ can be drawn as follows:

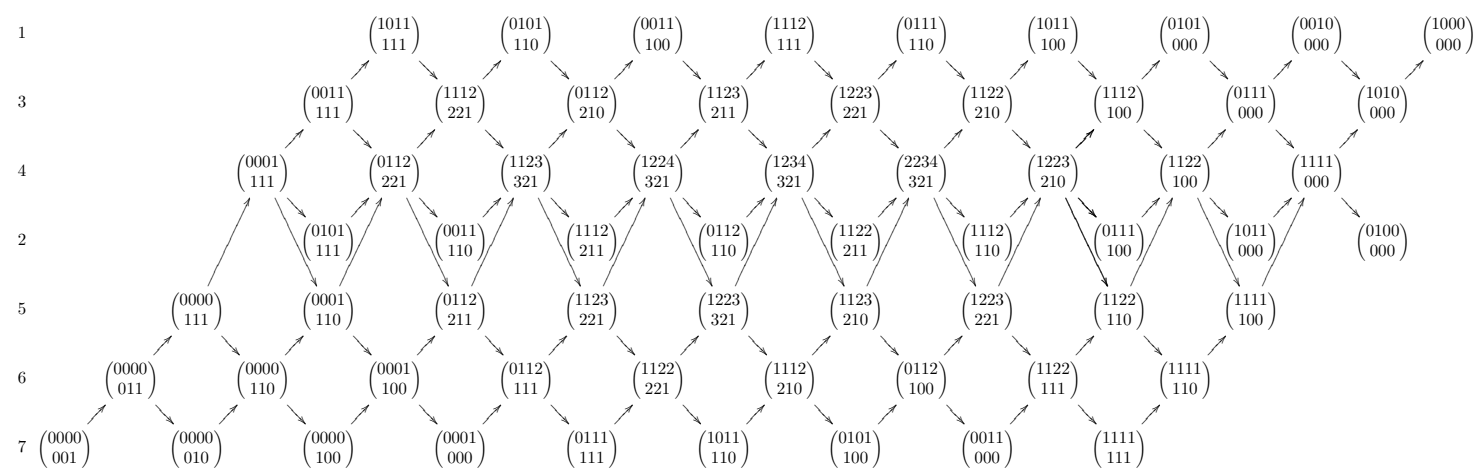




\section{Appendix B. Dynkin quiver $Q$ of type $E_{8}$ AND its AR Quiver $\Gamma_{Q}$}

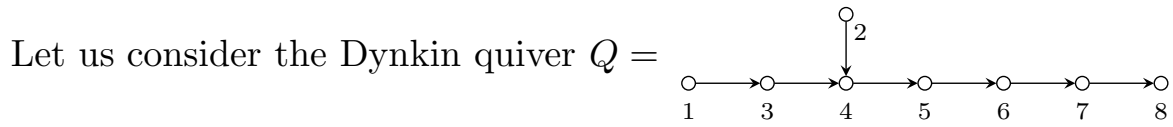

Its $\mathrm{AR}$ quiver $\Gamma_{Q}$ can be drawn as follows:

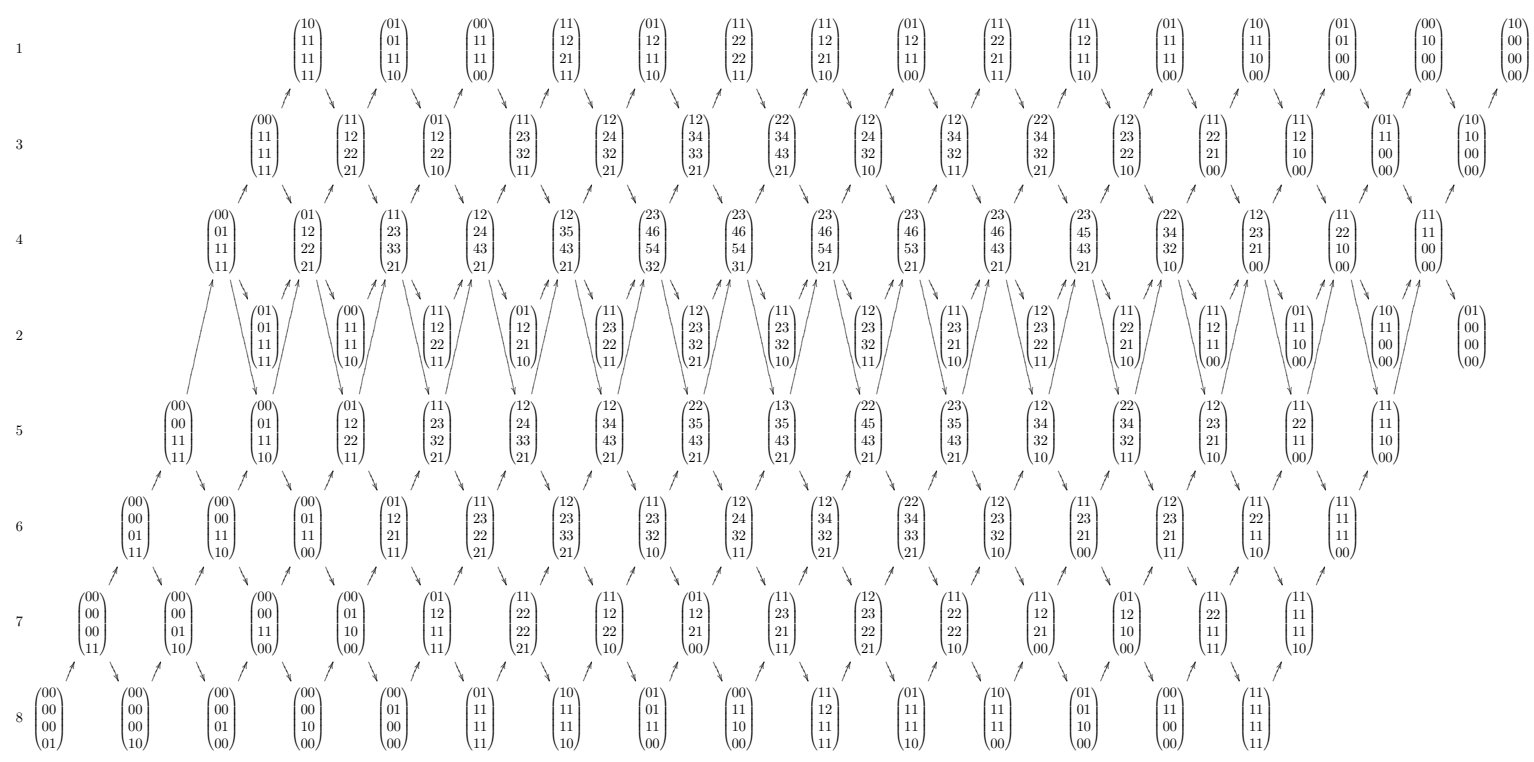

Appendix C. Denominator formulas for type $E_{7}^{(1)}$

We have determined the following denominator formulas:

$$
\begin{aligned}
& d_{1,1}(z)=\left(z-q^{2}\right)\left(z-q^{8}\right)\left(z-q^{12}\right)\left(z-q^{18}\right), \\
& d_{1,2}(z)=\left(z+q^{5}\right)\left(z+q^{9}\right)\left(z+q^{11}\right)\left(z+q^{15}\right), \\
& d_{1,3}(z)=\left(z+q^{3}\right)\left(z+q^{7}\right)\left(z+q^{9}\right)\left(z+q^{11}\right)\left(z+q^{13}\right)\left(z+q^{17}\right), \\
& d_{1,4}(z)=\left(z-q^{4}\right)\left(z-q^{6}\right)\left(z-q^{8}\right)\left(z-q^{10}\right)^{2}\left(z-q^{12}\right)\left(z-q^{14}\right)\left(z-q^{16}\right), \\
& d_{1,5}(z)=\left(z+q^{5}\right)\left(z+q^{7}\right)\left(z+q^{9}\right)\left(z+q^{11}\right)\left(z+q^{13}\right)\left(z+q^{15}\right), \\
& d_{1,6}(z)=\left(z-q^{6}\right)\left(z-q^{8}\right)\left(z-q^{12}\right)\left(z-q^{14}\right), \\
& d_{1,7}(z)=\left(z+q^{7}\right)\left(z+q^{13}\right), \\
& d_{2,2}(z)=\left(z-q^{2}\right)\left(z-q^{6}\right)\left(z-q^{8}\right)\left(z-q^{10}\right)\left(z-q^{12}\right)\left(z-q^{14}\right)\left(z-q^{18}\right), \\
& d_{2,3}(z)=\left(z-q^{4}\right)\left(z-q^{6}\right)\left(z-q^{8}\right)\left(z-q^{10}\right)^{2}\left(z-q^{12}\right)\left(z-q^{14}\right)\left(z-q^{16}\right), \\
& d_{2,4}(z)=\left(z+q^{3}\right)\left(z+q^{5}\right)\left(z+q^{7}\right)^{2}\left(z+q^{9}\right)^{2}\left(z+q^{11}\right)^{2}\left(z+q^{13}\right)^{2}\left(z+q^{15}\right)\left(z+q^{17}\right), \\
& d_{2,5}(z)=\left(z-q^{4}\right)\left(z-q^{6}\right)\left(z-q^{8}\right)^{2}\left(z-q^{10}\right)\left(z-q^{12}\right)^{2}\left(z-q^{14}\right)\left(z-q^{16}\right), \\
& d_{2,6}(z)=\left(z+q^{5}\right)\left(z+q^{7}\right)\left(z+q^{9}\right)\left(z+q^{11}\right)\left(z+q^{13}\right)\left(z+q^{15}\right), \\
& d_{2,7}(z)=\left(z-q^{6}\right)\left(z-q^{10}\right)\left(z-q^{14}\right), \\
& d_{3,3}(z)=\left(z-q^{2}\right)\left(z-q^{4}\right)\left(z-q^{6}\right)\left(z-q^{8}\right)^{2}\left(z-q^{10}\right)^{2}\left(z-q^{12}\right)^{2}\left(z-q^{14}\right)\left(z-q^{16}\right)\left(z-q^{18}\right),
\end{aligned}
$$




$$
\begin{aligned}
& d_{3,4}(z)=\left(z+q^{3}\right)\left(z+q^{5}\right)^{2}\left(z+q^{7}\right)^{2}\left(z+q^{9}\right)^{3}\left(z+q^{11}\right)^{3}\left(z+q^{13}\right)^{2}\left(z+q^{15}\right)^{2}\left(z+q^{17}\right), \\
& d_{3,5}(z)=\left(z-q^{4}\right)\left(z-q^{6}\right)^{2}\left(z-q^{8}\right)^{2}\left(z-q^{10}\right)^{2}\left(z-q^{12}\right)^{2}\left(z-q^{14}\right)^{2}\left(z-q^{16}\right), \\
& d_{3,6}(z)=\left(z+q^{5}\right)\left(z+q^{7}\right)^{2}\left(z+q^{9}\right)\left(z+q^{11}\right)\left(z+q^{13}\right)^{2}\left(z+q^{15}\right), \\
& d_{3,7}(z)=\left(z-q^{6}\right)\left(z-q^{8}\right)\left(z-q^{12}\right)\left(z-q^{14}\right), \\
& d_{4,4}(z)=\left(z-q^{2}\right)\left(z-q^{4}\right)^{2}\left(z-q^{6}\right)^{3}\left(z-q^{8}\right)^{4}\left(z-q^{10}\right)^{4}\left(z-q^{12}\right)^{4}\left(z-q^{14}\right)^{3}\left(z-q^{16}\right)^{2}\left(z-q^{18}\right), \\
& d_{4,5}(z)=\left(z+q^{3}\right)\left(z+q^{5}\right)^{2}\left(z+q^{7}\right)^{3}\left(z+q^{9}\right)^{3}\left(z+q^{11}\right)^{3}\left(z+q^{13}\right)^{3}\left(z+q^{15}\right)^{2}\left(z+q^{17}\right), \\
& d_{4,6}(z)=\left(z-q^{4}\right)\left(z-q^{6}\right)^{2}\left(z-q^{8}\right)^{2}\left(z-q^{10}\right)^{2}\left(z-q^{12}\right)^{2}\left(z-q^{14}\right)^{2}\left(z-q^{16}\right), \\
& d_{4,7}(z)=\left(z+q^{5}\right)\left(z+q^{7}\right)\left(z+q^{9}\right)\left(z+q^{11}\right)\left(z+q^{13}\right)\left(z+q^{15}\right), \\
& d_{5,5}(z)=\left(z-q^{2}\right)\left(z-q^{4}\right)\left(z-q^{6}\right)^{2}\left(z-q^{8}\right)^{2}\left(z-q^{10}\right)^{3}\left(z-q^{12}\right)^{2}\left(z-q^{14}\right)^{2}\left(z-q^{16}\right)\left(z-q^{18}\right), \\
& d_{5,6}(z)=\left(z+q^{3}\right)\left(z+q^{5}\right)\left(z+q^{7}\right)\left(z+q^{9}\right)^{2}\left(z+q^{11}\right)^{2}\left(z+q^{13}\right)\left(z+q^{15}\right)\left(z+q^{17}\right), \\
& d_{5,7}(z)=\left(z-q^{4}\right)\left(z-q^{8}\right)\left(z-q^{10}\right)\left(z-q^{12}\right)\left(z-q^{16}\right), \\
& d_{6,6}(z)=\left(z-q^{2}\right)\left(z-q^{4}\right)\left(z-q^{8}\right)\left(z-q^{10}\right)^{2}\left(z-q^{12}\right)\left(z-q^{16}\right)\left(z-q^{18}\right), \\
& d_{6,7}(z)=\left(z+q^{3}\right)\left(z+q^{9}\right)\left(z+q^{11}\right)\left(z+q^{17}\right), \\
& d_{7,7}(z)=\left(z-q^{2}\right)\left(z-q^{10}\right)\left(z-q^{18}\right) .
\end{aligned}
$$

\section{Appendix D. Denominator formulas for type $E_{8}^{(1)}$}

We have determined the following denominator formulas:

$$
\begin{aligned}
& d_{1,1}(z)=\left(z-q^{2}\right)\left(z-q^{8}\right)\left(z-q^{12}\right)\left(z-q^{14}\right)\left(z-q^{18}\right)\left(z-q^{20}\right)\left(z-q^{24}\right)\left(z-q^{30}\right), \\
& d_{1,2}(z)=\left(z+q^{5}\right)\left(z+q^{9}\right)\left(z+q^{11}\right)\left(z+q^{13}\right)\left(z+q^{15}\right)\left(z+q^{17}\right) \\
& \times\left(z+q^{19}\right)\left(z+q^{21}\right)\left(z+q^{23}\right)\left(z+q^{27}\right), \\
& d_{1,3}(z)=\left(z+q^{3}\right)\left(z+q^{7}\right)\left(z+q^{9}\right)\left(z+q^{11}\right)\left(z+q^{13}\right)^{2}\left(z+q^{15}\right)\left(z+q^{17}\right) \\
& \times\left(z+q^{19}\right)^{2}\left(z+q^{21}\right)\left(z+q^{23}\right)\left(z+q^{25}\right)\left(z+q^{29}\right), \\
& d_{1,4}(z)=\left(z-q^{4}\right)\left(z-q^{6}\right)\left(z-q^{8}\right)\left(z-q^{10}\right)^{2}\left(z-q^{12}\right)^{2}\left(z-q^{14}\right)^{2}\left(z-q^{16}\right)^{2} \\
& \times\left(z-q^{18}\right)^{2}\left(z-q^{20}\right)^{2}\left(z-q^{22}\right)^{2}\left(z-q^{24}\right)\left(z-q^{26}\right)\left(z-q^{28}\right), \\
& d_{1,5}(z)=\left(z+q^{5}\right)\left(z+q^{7}\right)\left(z+q^{9}\right)\left(z+q^{11}\right)^{2}\left(z+q^{13}\right)\left(z+q^{15}\right)^{2} \\
& \times\left(z+q^{17}\right)^{2}\left(z+q^{19}\right)\left(z+q^{21}\right)^{2}\left(z+q^{23}\right)\left(z+q^{25}\right)\left(z+q^{27}\right), \\
& d_{1,6}(z)=\left(z-q^{6}\right)\left(z-q^{8}\right)\left(z-q^{10}\right)\left(z-q^{12}\right)\left(z-q^{14}\right)\left(z-q^{16}\right)^{2}\left(z-q^{18}\right) \\
& \times\left(z-q^{20}\right)\left(z-q^{22}\right)\left(z-q^{24}\right)\left(z-q^{26}\right), \\
& d_{1,7}(z)=\left(z+q^{7}\right)\left(z+q^{9}\right)\left(z+q^{13}\right)\left(z+q^{15}\right)\left(z+q^{17}\right)\left(z+q^{19}\right)\left(z+q^{23}\right)\left(z+q^{25}\right) \text {, } \\
& d_{1,8}(z)=\left(z-q^{8}\right)\left(z-q^{14}\right)\left(z-q^{18}\right)\left(z-q^{24}\right) \text {, } \\
& d_{2,2}(z)=\left(z-q^{2}\right)\left(z-q^{6}\right)\left(z-q^{8}\right)\left(z-q^{10}\right)\left(z-q^{12}\right)^{2}\left(z-q^{14}\right) \\
& \times\left(z-q^{16}\right)^{2}\left(z-q^{18}\right)\left(z-q^{20}\right)^{2}\left(z-q^{22}\right)\left(z-q^{24}\right)\left(z-q^{26}\right)\left(z-q^{30}\right), \\
& d_{2,3}(z)=\left(z-q^{4}\right)\left(z-q^{6}\right)\left(z-q^{8}\right)\left(z-q^{10}\right)^{2}\left(z-q^{12}\right)^{2}\left(z-q^{14}\right)^{2}
\end{aligned}
$$




$$
\begin{aligned}
& \times\left(z-q^{16}\right)^{2}\left(z-q^{18}\right)^{2}\left(z-q^{20}\right)^{2}\left(z-q^{22}\right)^{2}\left(z-q^{24}\right)\left(z-q^{26}\right)\left(z-q^{28}\right), \\
& d_{2,4}(z)=\left(z+q^{3}\right)\left(z+q^{5}\right)\left(z+q^{7}\right)^{2}\left(z+q^{9}\right)^{2}\left(z+q^{11}\right)^{3}\left(z+q^{13}\right)^{3}\left(z+q^{15}\right)^{3}\left(z+q^{17}\right)^{3} \\
& \times\left(z+q^{19}\right)^{3}\left(z+q^{21}\right)^{3}\left(z+q^{23}\right)^{2}\left(z+q^{25}\right)^{2}\left(z+q^{27}\right)\left(z+q^{29}\right), \\
& d_{2,5}(z)=\left(z-q^{4}\right)\left(z-q^{6}\right)\left(z-q^{8}\right)^{2}\left(z-q^{10}\right)^{2}\left(z-q^{12}\right)^{2}\left(z-q^{14}\right)^{3}\left(z-q^{16}\right)^{2} \\
& \times\left(z-q^{18}\right)^{3}\left(z-q^{20}\right)^{2}\left(z-q^{22}\right)^{2}\left(z-q^{24}\right)^{2}\left(z-q^{26}\right)\left(z-q^{28}\right), \\
& d_{2,6}(z)=\left(z+q^{5}\right)\left(z+q^{7}\right)\left(z+q^{9}\right)^{2}\left(z+q^{11}\right)\left(z+q^{13}\right)^{2}\left(z+q^{15}\right)^{2} \\
& \times\left(z+q^{17}\right)^{2}\left(z+q^{19}\right)^{2}\left(z+q^{21}\right)\left(z+q^{23}\right)^{2}\left(z+q^{25}\right)\left(z+q^{27}\right), \\
& d_{2,7}(z)=\left(z-q^{6}\right)\left(z-q^{8}\right)\left(z-q^{10}\right)\left(z-q^{12}\right)\left(z-q^{14}\right)\left(z-q^{16}\right)^{2} \\
& \times\left(z-q^{18}\right)\left(z-q^{20}\right)\left(z-q^{22}\right)\left(z-q^{24}\right)\left(z-q^{26}\right), \\
& d_{2,8}(z)=\left(z+q^{7}\right)\left(z+q^{11}\right)\left(z+q^{15}\right)\left(z+q^{17}\right)\left(z+q^{21}\right)\left(z+q^{25}\right) \text {, } \\
& d_{3,3}(z)=\left(z-q^{2}\right)\left(z-q^{4}\right)\left(z-q^{6}\right)\left(z-q^{8}\right)^{2}\left(z-q^{10}\right)^{2}\left(z-q^{12}\right)^{3}\left(z-q^{14}\right)^{3}\left(z-q^{16}\right)^{2} \\
& \times\left(z-q^{18}\right)^{3}\left(z-q^{20}\right)^{3}\left(z-q^{22}\right)^{2}\left(z-q^{24}\right)^{2}\left(z-q^{26}\right)\left(z-q^{28}\right)\left(z-q^{30}\right), \\
& d_{3,4}(z)=\left(z+q^{3}\right)\left(z+q^{5}\right)^{2}\left(z+q^{7}\right)^{2}\left(z+q^{9}\right)^{3}\left(z+q^{11}\right)^{4}\left(z+q^{13}\right)^{4}\left(z+q^{15}\right)^{4} \\
& \times\left(z+q^{17}\right)^{4}\left(z+q^{19}\right)^{4}\left(z+q^{21}\right)^{4}\left(z+q^{23}\right)^{3}\left(z+q^{25}\right)^{2}\left(z+q^{27}\right)^{2}\left(z+q^{29}\right), \\
& d_{3,5}(z)=\left(z-q^{4}\right)\left(z-q^{6}\right)^{2}\left(z-q^{8}\right)^{2}\left(z-q^{10}\right)^{3}\left(z-q^{12}\right)^{3}\left(z-q^{14}\right)^{3}\left(z-q^{16}\right)^{4} \\
& \times\left(z-q^{18}\right)^{3}\left(z-q^{20}\right)^{3}\left(z-q^{22}\right)^{3}\left(z-q^{24}\right)^{2}\left(z-q^{26}\right)^{2}\left(z-q^{28}\right), \\
& d_{3,6}(z)=\left(z+q^{5}\right)\left(z+q^{7}\right)^{2}\left(z+q^{9}\right)^{2}\left(z+q^{11}\right)^{2}\left(z+q^{13}\right)^{2}\left(z+q^{15}\right)^{3}\left(z+q^{17}\right)^{3} \\
& \times\left(z+q^{19}\right)^{2}\left(z+q^{21}\right)^{2}\left(z+q^{23}\right)^{2}\left(z+q^{25}\right)^{2}\left(z+q^{27}\right), \\
& d_{3,7}(z)=\left(z-q^{6}\right)\left(z-q^{8}\right)^{2}\left(z-q^{10}\right)\left(z-q^{12}\right)\left(z-q^{14}\right)^{2}\left(z-q^{16}\right)^{2} \\
& \times\left(z-q^{18}\right)^{2}\left(z-q^{20}\right)\left(z-q^{22}\right)\left(z-q^{24}\right)^{2}\left(z-q^{26}\right), \\
& d_{3,8}(z)=\left(z+q^{7}\right)\left(z+q^{9}\right)\left(z+q^{13}\right)\left(z+q^{15}\right)\left(z+q^{17}\right)\left(z+q^{19}\right)\left(z+q^{23}\right)\left(z+q^{25}\right) \text {, } \\
& d_{4,4}(z)=\left(z-q^{2}\right)\left(z-q^{4}\right)^{2}\left(z-q^{6}\right)^{3}\left(z-q^{8}\right)^{4}\left(z-q^{10}\right)^{5}\left(z-q^{12}\right)^{6}\left(z-q^{14}\right)^{6}\left(z-q^{16}\right)^{6} \\
& \times\left(z-q^{18}\right)^{6}\left(z-q^{20}\right)^{6}\left(z-q^{22}\right)^{5}\left(z-q^{24}\right)^{4}\left(z-q^{26}\right)^{3}\left(z-q^{28}\right)^{2}\left(z-q^{30}\right), \\
& d_{4,5}(z)=\left(z+q^{3}\right)\left(z+q^{5}\right)^{2}\left(z+q^{7}\right)^{3}\left(z+q^{9}\right)^{4}\left(z+q^{11}\right)^{4}\left(z+q^{13}\right)^{5}\left(z+q^{15}\right)^{5} \\
& \times\left(z+q^{17}\right)^{5}\left(z+q^{19}\right)^{5}\left(z+q^{21}\right)^{4}\left(z+q^{23}\right)^{4}\left(z+q^{25}\right)^{3}\left(z+q^{27}\right)^{2}\left(z+q^{29}\right), \\
& d_{4,6}(z)=\left(z-q^{4}\right)\left(z-q^{6}\right)^{2}\left(z-q^{8}\right)^{3}\left(z-q^{10}\right)^{3}\left(z-q^{12}\right)^{3}\left(z-q^{14}\right)^{4}\left(z-q^{16}\right)^{4} \\
& \times\left(z-q^{18}\right)^{4}\left(z-q^{20}\right)^{3}\left(z-q^{22}\right)^{3}\left(z-q^{24}\right)^{3}\left(z-q^{26}\right)^{2}\left(z-q^{28}\right), \\
& d_{4,7}(z)=\left(z+q^{5}\right)\left(z+q^{7}\right)^{2}\left(z+q^{9}\right)^{2}\left(z+q^{11}\right)^{2}\left(z+q^{13}\right)^{2}\left(z+q^{15}\right)^{3}\left(z+q^{17}\right)^{3} \\
& \times\left(z+q^{19}\right)^{2}\left(z+q^{21}\right)^{2}\left(z+q^{23}\right)^{2}\left(z+q^{25}\right)^{2}\left(z+q^{27}\right), \\
& d_{4,8}(z)=\left(z-q^{6}\right)\left(z-q^{8}\right)\left(z-q^{10}\right)\left(z-q^{12}\right)\left(z-q^{14}\right)\left(z-q^{16}\right)^{2} \\
& \times\left(z-q^{18}\right)\left(z-q^{20}\right)\left(z-q^{22}\right)\left(z-q^{24}\right)\left(z-q^{26}\right), \\
& d_{5,5}(z)=\left(z-q^{2}\right)\left(z-q^{4}\right)\left(z-q^{6}\right)^{2}\left(z-q^{8}\right)^{3}\left(z-q^{10}\right)^{3}\left(z-q^{12}\right)^{4}\left(z-q^{14}\right)^{4}
\end{aligned}
$$




$$
\begin{gathered}
\quad \times\left(z-q^{16}\right)^{4}\left(z-q^{18}\right)^{4}\left(z-q^{20}\right)^{4}\left(z-q^{22}\right)^{3}\left(z-q^{24}\right)^{3}\left(z-q^{26}\right)^{2}\left(z-q^{28}\right), \\
\begin{aligned}
& d_{5,6}(z)=\left(z+q^{3}\right)\left(z+q^{5}\right)\left(z+q^{7}\right)^{2}\left(z+q^{9}\right)^{2}\left(z+q^{11}\right)^{3}\left(z+q^{13}\right)^{3}\left(z+q^{15}\right)^{3}\left(z+q^{17}\right)^{3} \\
& \times\left(z+q^{19}\right)^{3}\left(z+q^{21}\right)^{3}\left(z+q^{23}\right)^{2}\left(z+q^{25}\right)^{2}\left(z+q^{27}\right)\left(z+q^{29}\right), \\
& d_{5,7}(z)=\left(z-q^{4}\right)\left(z-q^{6}\right)\left(z-q^{8}\right)\left(z-q^{10}\right)^{2}\left(z-q^{12}\right)^{2}\left(z-q^{14}\right)^{2} \\
& \times\left(z-q^{16}\right)^{2}\left(z-q^{18}\right)^{2}\left(z-q^{20}\right)^{2}\left(z-q^{22}\right)^{2}\left(z-q^{24}\right)\left(z-q^{26}\right)\left(z-q^{28}\right), \\
& d_{5,8}(z)=\left(z+q^{5}\right)\left(z+q^{9}\right)\left(z+q^{11}\right)\left(z+q^{13}\right)\left(z+q^{15}\right) \\
& \times\left(z+q^{17}\right)\left(z+q^{19}\right)\left(z+q^{21}\right)\left(z+q^{23}\right)\left(z+q^{27}\right), \\
& d_{6,6}(z)=\left(z-q^{2}\right)\left(z-q^{4}\right)\left(z-q^{6}\right)\left(z-q^{8}\right)\left(z-q^{10}\right)^{2}\left(z-q^{12}\right)^{3}\left(z-q^{14}\right)^{2}\left(z-q^{16}\right)^{2} \\
& \times\left(z-q^{18}\right)^{2}\left(z-q^{20}\right)^{3}\left(z-q^{22}\right)^{2}\left(z-q^{24}\right)\left(z-q^{26}\right)\left(z-q^{28}\right)\left(z-q^{30}\right), \\
& d_{6,7}(z)=\left(z+q^{3}\right)\left(z+q^{5}\right)\left(z+q^{9}\right)\left(z+q^{11}\right)^{2}\left(z+q^{13}\right)^{2}\left(z+q^{15}\right)\left(z+q^{17}\right) \\
& \times\left(z+q^{19}\right)^{2}\left(z+q^{21}\right)^{2}\left(z+q^{23}\right)\left(z+q^{27}\right)\left(z+q^{29}\right), \\
& d_{6,8}(z)=\left(z-q^{4}\right)\left(z-q^{10}\right)\left(z-q^{12}\right)\left(z-q^{14}\right)\left(z-q^{18}\right)\left(z-q^{20}\right)\left(z-q^{22}\right)\left(z-q^{28}\right), \\
& d_{7,7}(z)=\left(z-q^{2}\right)\left(z-q^{4}\right)\left(z-q^{10}\right)\left(z-q^{12}\right)^{2}\left(z-q^{14}\right)\left(z-q^{18}\right)\left(z-q^{20}\right)^{2} \\
& \quad \times\left(z-q^{22}\right)\left(z-q^{28}\right)\left(z-q^{30}\right),
\end{aligned} \\
d_{7,8}(z)=\left(z+q^{3}\right)\left(z+q^{11}\right)\left(z+q^{13}\right)\left(z+q^{19}\right)\left(z+q^{21}\right)\left(z+q^{29}\right), \\
d_{8,8}(z)=\left(z-q^{2}\right)\left(z-q^{12}\right)\left(z-q^{20}\right)\left(z-q^{30}\right) .
\end{gathered}
$$

\section{Appendix E. Addendum - Removing Ambiguities}

The following has been included as an addendum to our publication [OS19], where we remove the ambiguities from Proposition 4.29, Proposition 7.8 and that were previously in Appendix C and Appendix D in the published version.

Proposition E.1 ([Oh19, Proposition 6.8]). Let $Q$ be a Dynkin quiver of finite simply-laced type. For a $[Q]$-minimal pair $(\alpha, \beta)$ of a simple sequence $\underline{s}=\left(\alpha_{1}, \ldots, \alpha_{k}\right), V_{Q}^{(1)}\left(\alpha_{1}\right) \otimes \cdots \otimes V_{Q}^{(1)}\left(\alpha_{k}\right)$ is simple and there exists a surjective homomorphism

$$
V_{Q}^{(1)}(\beta) \otimes V_{Q}^{(1)}(\alpha) \rightarrow V_{Q}^{(1)}\left(\alpha_{1}\right) \otimes \cdots \otimes V_{Q}^{(1)}\left(\alpha_{k}\right) .
$$

We first resolve the ambiguity in Proposition 4.29.

Proposition E.2. For type $E_{6}^{(1)}$, we have

$$
d_{4,4}(z)=\left(z-q^{2}\right)\left(z-q^{4}\right)^{2}\left(z-q^{6}\right)^{3}\left(z-q^{8}\right)^{3}\left(z-q^{10}\right)^{2}\left(z-q^{12}\right) .
$$

Proof. Note that, from the AR-quiver of type $E_{6}$ in (2.6), we can read that

$$
\left(\left(\begin{array}{l}
111 \\
100
\end{array}\right),\left(\begin{array}{l}
101 \\
110
\end{array}\right)\right) \text { is a }[Q] \text {-minimal pair of the simple sequence }\left(\left(\begin{array}{l}
101 \\
100
\end{array}\right),\left(\begin{array}{l}
111 \\
110
\end{array}\right)\right) \text {. }
$$

Hence Proposition E.1 states that we have a homomorphism

$$
V\left(\varpi_{1}\right)_{(-q)^{-3}} \otimes V\left(\varpi_{4}\right)_{(-q)^{1}} \rightarrow V\left(\varpi_{2}\right) \otimes V\left(\varpi_{5}\right) .
$$


Then we have

$$
\frac{d_{1,4}\left((-q)^{-3} z\right) d_{4,4}\left((-q)^{1} z\right)}{d_{2,4}(z) d_{5,4}(z)} \times \frac{a_{2,4}(z) d_{5,4}(z)}{a_{1,4}\left((-q)^{-3} z\right) a_{4,4}\left((-q)^{1} z\right)} \in \mathbf{k}\left[z^{ \pm 1}\right] .
$$

by [AK97, Lemma C.15]. From Lemma 3.9, one can compute that

$$
\begin{array}{rlrl}
a_{2,4}(z) & \equiv \frac{[19][5][23][1]}{[7][17][11][13]} & a_{3,4}(z) \equiv a_{4,5}(z) \equiv \frac{[1][3][21][23]}{[9][11][13][15]} \\
a_{1,4}(z) \equiv \frac{[2][22]}{[10][14]} & a_{4,4}(z) \equiv \frac{[0][2][4][20][22][24]}{[8][10][12]^{2}[14][16]}
\end{array}
$$

Since we have computed $d_{1,4}(z), d_{2,4}(z)$ and $d_{5,4}(z)$, (E.2) can be written as follows:

$$
\frac{d_{1,4}\left((-q)^{-3} z\right) d_{4,4}\left((-q)^{1} z\right)}{d_{2,4}(z) d_{5,4}(z)}=\frac{\left(z+q^{1}\right)\left(z+q^{3}\right)^{2}\left(z+q^{5}\right)^{2} \text { or }{ }^{3}\left(z+q^{7}\right)^{4}\left(z-q^{9}\right)^{3}\left(z-q^{11}\right)^{2}\left(z+q^{13}\right)}{\left(z+q^{3}\right)^{2}\left(z+q^{5}\right)^{3}\left(z+q^{7}\right)^{4}\left(z+q^{9}\right)^{3}\left(z+q^{11}\right)^{2}}
$$

by Proposition 4.29 and

$$
\begin{aligned}
\frac{a_{2,4}(z) d_{5,4}(z)}{a_{1,4}\left((-q)^{-3} z\right) a_{4,4}\left((-q)^{1} z\right)} & =\frac{[19][5][23][1]}{[7][17][11][13]} \times \frac{[1][3][21][23]}{[9][11][13][15]} \times \frac{[7][11]}{[-1][19]} \times \frac{[9][11][13]^{2}[15][17]}{[1][3][5][21][23][25]} \\
& =\frac{[23][1]}{[-1][25]}=\frac{\left(z-(-q)^{-1}\right)}{\left(z-(-q)^{1}\right)} .
\end{aligned}
$$

Thus we have

$$
\begin{aligned}
& \frac{\left(z+q^{1}\right)\left(z+q^{3}\right)^{2}\left(z+q^{5}\right)^{2} \text { or }{ }^{3}\left(z+q^{7}\right)^{4}\left(z-q^{9}\right)^{3}\left(z-q^{11}\right)^{2}\left(z+q^{13}\right)}{\left(z+q^{3}\right)^{2}\left(z+q^{5}\right)^{3}\left(z+q^{7}\right)^{4}\left(z+q^{9}\right)^{3}\left(z+q^{11}\right)^{2}} \times \frac{\left(z-(-q)^{-1}\right)}{\left(z-(-q)^{1}\right)} \\
& =\frac{\left(z+q^{-1}\right)\left(z+q^{3}\right)^{2}\left(z+q^{5}\right)^{2} \text { or } 3\left(z+q^{7}\right)^{4}\left(z-q^{9}\right)^{3}\left(z-q^{11}\right)^{2}\left(z+q^{13}\right)}{\left(z+q^{3}\right)^{2}\left(z+q^{5}\right)^{3}\left(z+q^{7}\right)^{4}\left(z+q^{9}\right)^{3}\left(z+q^{11}\right)^{2}} \in \mathbf{k}\left[z^{ \pm 1}\right] .
\end{aligned}
$$

which implies that the order of degree $(-q)^{6}$ should be 3 .

By applying generalized Schur-Weyl duality

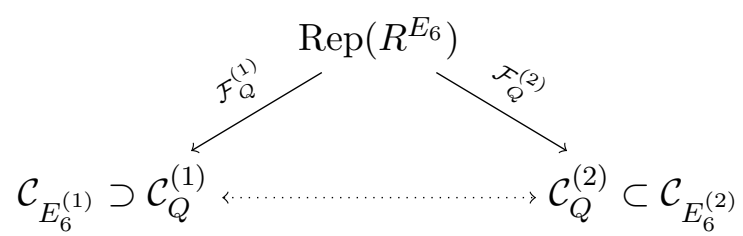

we have the twisted analogue of Proposition E.1:

Proposition E.3. Let $Q$ be a Dynkin quiver of finite simply-laced type. For a $[Q]$-minimal pair $(\alpha, \beta)$ of a simple sequence $\underline{s}=\left(\alpha_{1}, \ldots, \alpha_{k}\right), V_{Q}^{(2)}\left(\alpha_{1}\right) \otimes \cdots \otimes V_{Q}^{(2)}\left(\alpha_{k}\right)$ is simple and there exists a surjective homomorphism

$$
V_{Q}^{(2)}(\beta) \otimes V_{Q}^{(2)}(\alpha) \rightarrow V_{Q}^{(2)}\left(\alpha_{1}\right) \otimes \cdots \otimes V_{Q}^{(2)}\left(\alpha_{k}\right) .
$$

In particular, (E.1) transfers to the following homomorphism in $\mathcal{C}_{Q}^{(2)}$ under the map in Proposition 6.5:

$$
V\left(\varpi_{1}\right)_{(-q)^{-3}} \otimes V\left(\varpi_{3}\right)_{\sqrt{-1}(-q)^{1}} \rightarrow V\left(\varpi_{2}\right)_{-1} \otimes V\left(\varpi_{4}\right)_{\sqrt{-1}} .
$$

Next, we resolve the ambiguity in Proposition 7.8. 
Proposition E.4. For type $E_{6}^{(2)}$, we have

$$
d_{3,3}(z)=\left(z^{2}-q^{4}\right)\left(z^{2}-q^{8}\right)^{2}\left(z^{2}-q^{12}\right)^{3}\left(z^{2}-q^{16}\right)^{3}\left(z^{2}-q^{20}\right)^{2}\left(z^{2}-q^{24}\right) .
$$

Proof. By (E.3) and [AK97, Lemma C.15], we have

$$
\frac{d_{1,3}\left(\sqrt{-1}(-q)^{-3} z\right) d_{3,3}\left((-q)^{1} z\right)}{d_{2,3}(i z) d_{3,4}(z)} \times \frac{a_{2,3}(\sqrt{-1} z) a_{3,4}(z)}{a_{1,3}\left(\sqrt{-1}(-q)^{-3} z\right) a_{3,3}\left((-q)^{1} z\right)} \in \mathbf{k}\left[z^{ \pm 1}\right] .
$$

From Lemma 3.9, one can compute that

$$
\begin{array}{rlrl}
a_{2,3}(\sqrt{-1} z) & \equiv \frac{\{3\}\{21\}\{1\}\{23\}}{\{9\}\{15\}\{11\}\{13\}}, & a_{3,4}(z) \equiv \frac{\{5\}\{19\}\{1\}\{23\}}{\{7\}\{17\}\{11\}\{13\}}, \\
a_{1,3}(\sqrt{-1} z) \equiv \frac{\{2\}\{22\}}{\{10\}\{14\}}, & a_{3,3}(z) \equiv \frac{\{4\}\{20\}\{2\}\{22\}\{0\}\{24\}}{\{8\}\{16\}\{10\}\{14\}\{12\}^{2}} .
\end{array}
$$

Since we have computed $d_{1,3}(z), d_{2,3}(z)$ and $d_{3,4}(z)$, (E.4) can be written as follows:

$$
\begin{aligned}
& \frac{d_{1,3}\left(\sqrt{-1}(-q)^{-3} z\right) d_{3,3}\left((-q)^{1} z\right)}{d_{2,3}(\sqrt{-1} z) d_{3,4}(z)} \\
& =\frac{\left(z^{2}-q^{2}\right)\left(z^{2}-q^{6}\right)^{2}\left(z^{2}-q^{10}\right)^{2} \text { or } 3\left(z^{2}-q^{14}\right)^{4}\left(z^{2}-q^{18}\right)^{3}\left(z^{2}-q^{22}\right)^{2}\left(z^{2}-q^{26}\right)}{\left(z^{2}-q^{6}\right)^{2}\left(z^{2}-q^{10}\right)^{3}\left(z^{2}-q^{14}\right)^{4}\left(z^{2}-q^{18}\right)^{3}\left(z^{2}-q^{22}\right)^{2}}
\end{aligned}
$$

by Proposition 7.8 and

$$
\begin{aligned}
& \frac{a_{2,3}(\sqrt{-1} z) a_{3,4}(z)}{a_{1,3}\left(\sqrt{-1}(-q)^{-3} z\right) a_{3,3}\left((-q)^{1} z\right)} \\
& =\frac{\{3\}\{21\}\{1\}\{23\}}{\{9\}\{15\}\{11\}\{13\}} \times \frac{\{5\}\{19\}\{1\}\{23\}}{\{7\}\{17\}\{11\}\{13\}} \times \frac{\{7\}\{11\}}{\{-1\}\{19\}} \times \frac{\{9\}\{17\}\{11\}\{15\}\{13\}^{2}}{\{5\}\{21\}\{3\}\{23\}\{1\}\{25\}} \\
& =\frac{\{1\}\{23\}}{\{25\}\{-1\}}=\frac{\left(z^{2}-q^{-2}\right)}{\left(z^{2}-q^{2}\right)} .
\end{aligned}
$$

Thus we have

$$
\frac{\left(z^{2}-q^{-2}\right)\left(z^{2}-q^{6}\right)^{2}\left(z^{2}-q^{10}\right)^{2} \text { or } 3\left(z^{2}-q^{14}\right)^{4}\left(z^{2}-q^{18}\right)^{3}\left(z^{2}-q^{22}\right)^{2}\left(z^{2}-q^{26}\right)}{\left(z^{2}-q^{6}\right)^{2}\left(z^{2}-q^{10}\right)^{3}\left(z^{2}-q^{14}\right)^{4}\left(z^{2}-q^{18}\right)^{3}\left(z^{2}-q^{22}\right)^{2}} \in \mathbf{k}\left[z^{ \pm 1}\right],
$$

which implies our assertion.

For types $E_{7}^{(1)}$ and $E_{8}^{(1)}$, we can remove the ambiguities in the previous version by applying the same arguments as above. Hence there are roots of order 4,5,6 also. To the best knowledge of the authors, this is the first such observation of a root of order strictly larger than 3 .

When we prove for $E_{7}^{(1)}$-cases, we employ the following homomorphisms

$$
\begin{aligned}
& V(3)_{(-q)^{-1}} \otimes V(6)_{(-q)^{4}} \rightarrow V(1) \otimes V(2)_{(-q)} \otimes V(7)_{(-q)^{3}}, \\
& V(3)_{(-q)^{-1}} \otimes V(5)_{(-q)^{3}} \rightarrow V(1) \otimes V(2)_{(-q)} \otimes V(6)_{(-q)^{2}}, \\
& V(1)_{(-q)^{-3}} \otimes V(4)_{(-q)^{1}} \rightarrow V(2) \otimes V(5),
\end{aligned}
$$

which can be obtained from Proposition E.1 and Table 1. 


\begin{tabular}{cccc}
\hline Minimal pair $\underline{p}$ & $\left(\left(\begin{array}{c}1111 \\
110\end{array}\right),\left(\begin{array}{c}1223 \\
221\end{array}\right)\right)$ & $\left(\left(\begin{array}{c}1122 \\
110\end{array}\right),\left(\begin{array}{c}1223 \\
221\end{array}\right)\right)$ & $\left(\left(\begin{array}{c}1224 \\
321\end{array}\right),\left(\begin{array}{c}1011 \\
111\end{array}\right)\right)$ \\
\hline $\operatorname{soc}_{Q}(\underline{p})$ & $\left(\left(\begin{array}{c}0111 \\
110\end{array}\right),\left(\begin{array}{c}1112 \\
110\end{array}\right),\left(\begin{array}{c}1111 \\
111\end{array}\right)\right)$ & $\left(\left(\begin{array}{c}0111 \\
110\end{array}\right),\left(\begin{array}{c}1112 \\
110\end{array}\right),\left(\begin{array}{c}1122 \\
111\end{array}\right)\right)$ & $\left(\left(\begin{array}{c}1112 \\
211\end{array}\right),\left(\begin{array}{c}1123 \\
221\end{array}\right)\right)$ \\
\hline
\end{tabular}

TABLE 1. The minimal pairs $\underline{p}$ and their $\operatorname{socles} \operatorname{soc}_{Q}(\underline{p})$ of $\Gamma_{Q}$ used to remove ambiguities.

\section{REFERENCES}

[AK97] Tatsuya Akasaka and Masaki Kashiwara. Finite-dimensional representations of quantum affine algebras. Publ. Res. Inst. Math. Sci., 33(5):839-867, 1997.

[B9́9] Robert Bédard. On commutation classes of reduced words in Weyl groups. European J. Combin., 20(6):483-505, 1999.

[BFKL06] Georgia Benkart, Igor Frenkel, Seok-Jin Kang, and Hyeonmi Lee. Level 1 perfect crystals and path realizations of basic representations at $q=0$. Int. Math. Res. Not., pages Art. ID 10312, 28, 2006.

[BKM14] Jonathan Brundan, Alexander Kleshchev, and Peter J. McNamara. Homological properties of finite-type Khovanov-Lauda-Rouquier algebras. Duke Math. J., 163(7):1353-1404, 2014.

[BL91] Denis Bernard and André LeClair. Quantum group symmetries and nonlocal currents in 2D QFT. Comm. Math. Phys., 142(1):99-138, 1991.

[Cha01] Vyjayanthi Chari. On the fermionic formula and the Kirillov-Reshetikhin conjecture. Int. Math. Res. Not. IMRN, (12):629-654, 2001.

[Cha02] Vyjayanthi Chari. Braid group actions and tensor products. Int. Math. Res. Not., (7):357-382, 2002.

[CP95] Vyjayanthi Chari and Andrew Pressley. Quantum affine algebras and their representations. In Representations of groups (Banff, AB, 1994), volume 16 of CMS Conf. Proc., pages 59-78. Amer. Math. Soc., Providence, RI, 1995.

[CP96] Vyjayanthi Chari and Andrew Pressley. Yangians, integrable quantum systems and Dorey's rule. Comm. Math. Phys., 181(2):265-302, 1996.

[Dev17] The Sage Developers. Sage Mathematics Software (Version 8.1). The Sage Development Team, 2017. http://www. sagemath.org.

[DO94] Etsurō Date and Masato Okado. Calculation of excitation spectra of the spin model related with the vector representation of the quantized affine algebra of type $A_{n}^{(1)}$. Internat. J. Modern Phys. A, 9(3):399417, 1994.

[Dor91] Patrick Dorey. Root systems and purely elastic S-matrices. Nuclear Phys. B, 358(3):654-676, 1991.

[EFK98] Pavel I. Etingof, Igor B. Frenkel, and Alexander A. Kirillov, Jr. Lectures on representation theory and Knizhnik-Zamolodchikov equations, volume 58 of Mathematical Surveys and Monographs. American Mathematical Society, Providence, RI, 1998.

[FH11a] Edward Frenkel and David Hernandez. Langlands duality for finite-dimensional representations of quantum affine algebras. Lett. Math. Phys., 96(1-3):217-261, 2011.

[FH11b] Edward Frenkel and David Hernandez. Langlands duality for representations of quantum groups. Math. Ann., 349(3):705-746, 2011.

$\left[\mathrm{FH}^{+} 15\right] \quad$ Edward Frenkel, David Hernandez, et al. Baxters relations and spectra of quantum integrable models. Duke Math. J., 164(12):2407-2460, 2015.

[FR98] Edward Frenkel and Nicolai Reshetikhin. Deformations of $W$-algebras associated to simple Lie algebras. Comm. Math. Phys., 197(1):1-32, 1998.

[FR99] Edward Frenkel and Nicolai Reshetikhin. The $q$-characters of representations of quantum affine algebras and deformations of $\mathscr{W}$-algebras. In Recent developments in quantum affine algebras and related topics (Raleigh, NC, 1998), volume 248 of Contemp. Math., pages 163-205. Amer. Math. Soc., Providence, RI, 1999.

[Fuj18] Ryo Fujita. Geometric realization of Dynkin quiver type quantum affine Schur-Weyl duality. Preprint, arXiv:1803.01538, 2018.

[GRV94] Victor Ginzburg, Nicolai Reshetikhin, and Éric Vasserot. Quantum groups and flag varieties. In Mathematical aspects of conformal and topological field theories and quantum groups (South Hadley, MA, 1992), volume 175 of Contemp. Math., pages 101-130. Amer. Math. Soc., Providence, RI, 1994. 
[Her06] David Hernandez. The Kirillov-Reshetikhin conjecture and solutions of T-systems. J. Reine Angew. Math., 596:63-87, 2006.

[Her10] David Hernandez. Kirillov-Reshetikhin conjecture: the general case. Int. Math. Res. Not. IMRN, (1):149193, 2010.

[HL10] David Hernandez and Bernard Leclerc. Cluster algebras and quantum affine algebras. Duke Math. J., 154(2):265-341, 2010.

[HL15] David Hernandez and Bernard Leclerc. Quantum Grothendieck rings and derived Hall algebras. J. Reine Angew. Math., 701:77-126, 2015.

[HL16] David Hernandez and Bernard Leclerc. A cluster algebra approach to q-characters of Kirillov-Reshetikhin modules. J. Eur. Math. Soc. (JEMS), 18(5):1113-1159, 2016.

[HN06] David Hernandez and Hiraku Nakajima. Level 0 monomial crystals. Nagoya Math. J., 184:85-153, 2006.

[IKT12] Rei Inoue, Atsuo Kuniba, and Taichiro Takagi. Integrable structure of box-ball systems: crystal, Bethe ansatz, ultradiscretization and tropical geometry. J. Phys. A, 45(7):073001, 64, 2012.

[Kac90] Victor G. Kac. Infinite-dimensional Lie algebras. Cambridge University Press, Cambridge, third edition, 1990.

[Kas90] Masaki Kashiwara. Crystalizing the $q$-analogue of universal enveloping algebras. Comm. Math. Phys., 133(2):249-260, 1990.

[Kas91] Masaki Kashiwara. On crystal bases of the $q$-analogue of universal enveloping algebras. Duke Math. J., 63(2):465-516, 1991.

[Kas02] Masaki Kashiwara. On level-zero representations of quantized affine algebras. Duke Math. J., 112(1):117175, 2002.

[Kat14] Syu Kato. Poincaré-Birkhoff-Witt bases and Khovanov-Lauda-Rouquier algebras. Duke Math. J., 163(3):619-663, 2014.

[KKK15] Seok-Jin Kang, Masaki Kashiwara, and Myungho Kim. Symmetric quiver Hecke algebras and R-matrices of quantum affine algebras, II. Duke Math. J., 164(8):1549-1602, 2015.

[KKK18] Seok-Jin Kang, Masaki Kashiwara, and Myungho Kim. Symmetric quiver Hecke algebras and $R$-matrices of quantum affine algebras. Invent. Math., 211(2):591-685, 2018.

[KKKO15a] Seok-Jin Kang, Masaki Kashiwara, Myungho Kim, and Se-jin Oh. Simplicity of heads and socles of tensor products. Compos. Math., 151(2):377-396, 2015.

[KKKO15b] Seok-Jin Kang, Masaki Kashiwara, Myungho Kim, and Se-jin Oh. Symmetric quiver Hecke algebras and $R$-matrices of quantum affine algebras III. Proc. Lond. Math. Soc. (3), 111(2):420-444, 2015.

[KKKO16] Seok-Jin Kang, Masaki Kashiwara, Myungho Kim, and Se-jin Oh. Symmetric quiver Hecke algebras and $R$-matrices of quantum affine algebras IV. Selecta Math. (N.S.), 22(4):1987-2015, 2016.

[KKO17] Masaki Kashiwara, Myungho Kim, and Se-jin Oh. Monoidal categories of modules over quantum affine algebras of type A and B. Proceedings of the London Mathematical Society, 2017.

[KL09] Mikhail Khovanov and Aaron D. Lauda. A diagrammatic approach to categorification of quantum groups. I. Represent. Theory, 13:309-347, 2009.

[KL11] Mikhail Khovanov and Aaron D. Lauda. A diagrammatic approach to categorification of quantum groups II. Trans. Amer. Math. Soc., 363(5):2685-2700, 2011.

[Kle98] Michael Steven Kleber. Finite dimensional representations of quantum affine algebras. ProQuest LLC, Ann Arbor, MI, 1998. Thesis (Ph.D.)-University of California, Berkeley.

[KM94] Seok-Jin Kang and Kailash C. Misra. Crystal bases and tensor product decompositions of $U_{q}\left(G_{2}\right)$ modules. J. Algebra, 163(3):675-691, 1994.

[KMOY07] M. Kashiwara, K. C. Misra, M. Okado, and D. Yamada. Perfect crystals for $U_{q}\left(D_{4}^{(3)}\right)$. J. Algebra, 317(1):392-423, 2007.

[KNS11] Atsuo Kuniba, Tomoki Nakanishi, and Junji Suzuki. T-systems and $Y$-systems in integrable systems. $J$. Phys. A, 44(10):103001, 146, 2011.

[KO18] Masaki Kashiwara and Se-jin Oh. Categorical relations between langlands dual quantum affine algebras: doubly laced types. Journal of Algebraic Combinatorics, Jun 2018.

[KR11] Alexander Kleshchev and Arun Ram. Representations of Khovanov-Lauda-Rouquier algebras and combinatorics of Lyndon words. Math. Ann., 349(4):943-975, 2011.

[Lus90] George Lusztig. Canonical bases arising from quantized enveloping algebras. Journal of the American Mathematical Society, 3(2):447-498, 1990. 
[McN15] Peter J. McNamara. Finite dimensional representations of Khovanov-Lauda-Rouquier algebras I: Finite type. J. Reine Angew. Math., 707:103-124, 2015.

[MOW12] Kailash C. Misra, Masato Okado, and Evan A. Wilson. Soliton cellular automaton associated with $G_{2}^{(1)}$ crystal base. J. Math. Phys., 53(1):013510, 21, 2012.

[Nak04] Hiraku Nakajima. Quiver varieties and $t$-analogs of $q$-characters of quantum affine algebras. Ann. of Math. (2), 160(3):1057-1097, 2004.

[NS03] Satoshi Naito and Daisuke Sagaki. Path model for a level-zero extremal weight module over a quantum affine algebra. Int. Math. Res. Not., (32):1731-1754, 2003.

[NS05] Satoshi Naito and Daisuke Sagaki. Crystal of Lakshmibai-Seshadri paths associated to an integral weight of level zero for an affine Lie algebra. Int. Math. Res. Not., (14):815-840, 2005.

[NS06a] Satoshi Naito and Daisuke Sagaki. Construction of perfect crystals conjecturally corresponding to Kirillov-Reshetikhin modules over twisted quantum affine algebras. Comm. Math. Phys., 263(3):749787, 2006.

[NS06b] Satoshi Naito and Daisuke Sagaki. Path model for a level-zero extremal weight module over a quantum affine algebra. II. Adv. Math., 200(1):102-124, 2006.

[Oh15] Se-jin Oh. The denominators of normalized $R$-matrices of types $A_{2 n-1}^{(2)}, A_{2 n}^{(2)}, B_{n}^{(1)}$ and $D_{n+1}^{(2)}$. Publ. Res. Inst. Math. Sci., 51(4):709-744, 2015.

[Oh16] Se-jin Oh. Auslander-Reiten quiver of type D and generalized quantum affine Schur-Weyl duality. J. Algebra, 460:203-252, 2016.

[Oh17] Se-jin Oh. Auslander-Reiten quiver of type A and generalized quantum affine Schur-Weyl duality. Trans. Amer. Math. Soc., 369(3):1895-1933, 2017.

[Oh19] Se-jin Oh. Auslander-Reiten quiver and representation theories related to KLR-type Schur-Weyl duality. Math. Z., (1-2):499-554, 2019.

[OS15] Se-jin Oh and Uhi Rinn Suh. Combinatorial Auslander-Reiten quivers and reduced expressions. Preprint, arXiv: 1509.04820, 2015.

[OS16a] Se-jin Oh and Uhi Rinn Suh. Twisted coxeter elements and folded AR-quivers via Dynkin diagram automorphisms: I. Preprint, arXiv:1606.00076, 2016.

[OS16b] Se-jin Oh and Uhi Rinn Suh. Twisted Coxeter elements and folded AR-quivers via Dynkin diagram automorphisms: II. Preprint, arXiv:1606.00102, 2016.

[OS19] Se-jin Oh and Travis Scrimshaw. Categorical relations between langlands dual quantum affine algebras: Exceptional cases. Comm. Math. Phys., 368(1):295-367, 2019.

[OSS03] Masato Okado, Anne Schilling, and Mark Shimozono. Virtual crystals and Kleber's algorithm. Comm. Math. Phys., 238(1-2):187-209, 2003.

[Rou08] Raphaël Rouquier. 2-Kac-Moody algebras, 2008. arXiv:0812.5023.

[Rou12] Raphaël Rouquier. Quiver Hecke algebras and 2-Lie algebras. Algebra Colloq., 19(2):359-410, 2012.

[SCc08] The Sage-Combinat community. Sage-Combinat: enhancing Sage as a toolbox for computer exploration in algebraic combinatorics, 2008. http://combinat.sagemath.org.

[Ste01] John R. Stembridge. Minuscule elements of Weyl groups. J. Algebra, 235(2):722-743, 2001.

[VV02] M. Varagnolo and E. Vasserot. Standard modules of quantum affine algebras. Duke Math. J., 111(3):509533, 2002.

[VV11] M. Varagnolo and E. Vasserot. Canonical bases and KLR-algebras. J. Reine Angew. Math., 659:67-100, 2011.

[Yam98] Shigenori Yamane. Perfect crystals of $U_{q}\left(G_{2}^{(1)}\right)$. J. Algebra, 210(2):440-486, 1998.

[YZ11] C. A. S. Young and R. Zegers. Dorey's rule and the $q$-characters of simply-laced quantum affine algebras. Comm. Math. Phys., 302(3):789-813, 2011. 
(S.-j. Oh) Ewha Womans University Seoul, 52 Ewhayeodae-gil, Daehyeon-dong, Seodaemun-Gu, Seoul, South Korea

E-mail address: sejin092@gmail.com

URL: https://sites.google.com/site/mathsejinoh/

(T. Scrimshaw) School of Mathematics and Physics, The University of Queensland, St. Lucia, QLD 4072, Australia

E-mail address: tcscrims@gmail.com

URL: https://people.smp.uq.edu.au/TravisScrimshaw/ 